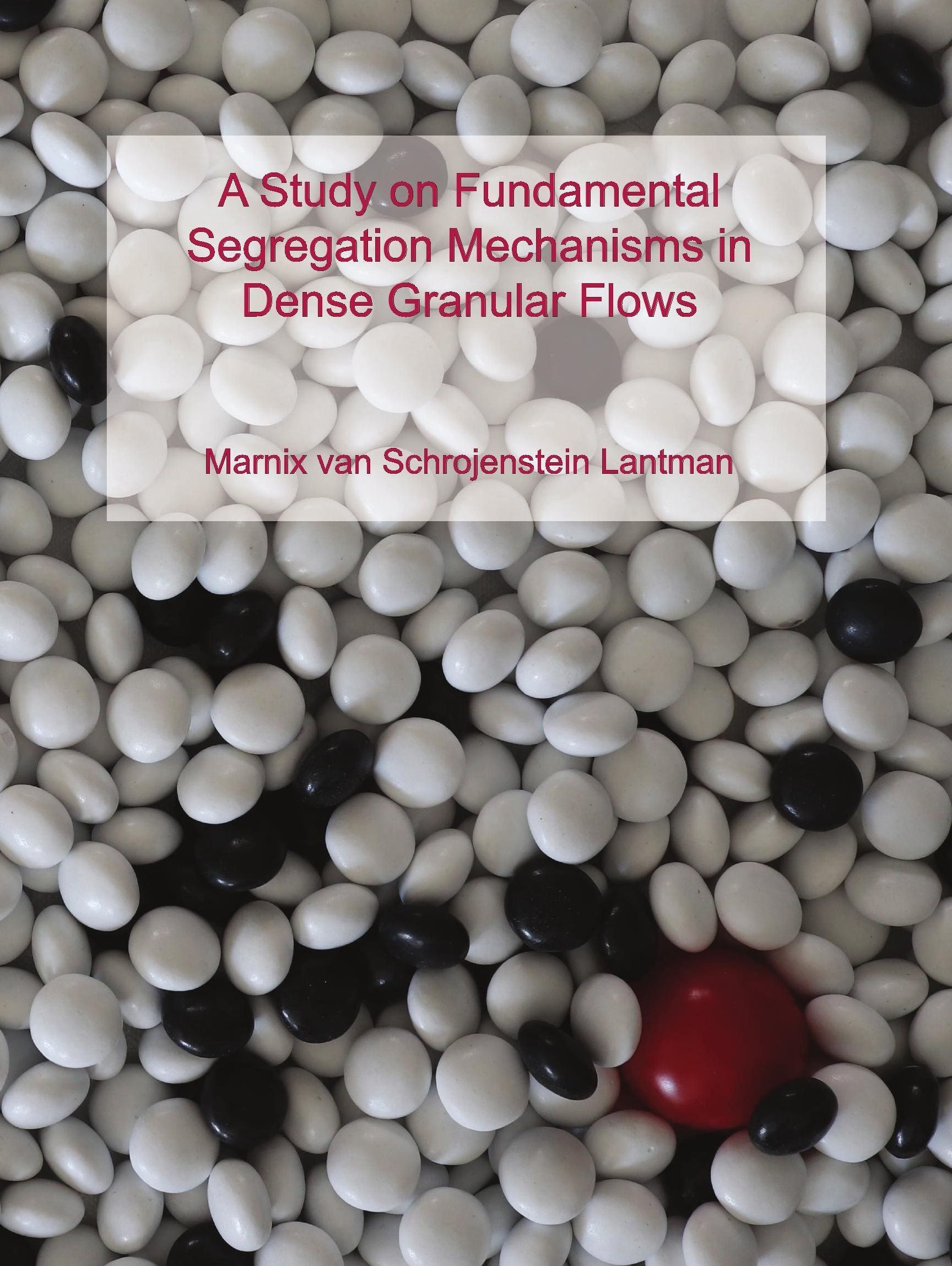




\section{A STUDY ON}

\section{Fundamental SEgregation MEChanisms in Dense Granular Flows}

Marnix Pieter van Schrojenstein Lantman 


\section{Thesis committee members:}

Chair:

Prof.dr. G. P. M. R. Dewulf,

Universiteit Twente

Promotor:

Prof.dr. A. R. Thornton,

Universiteit Twente

Co-promotor:

Prof.dr.rer.-nat. S. Luding,

Universiteit Twente

Commission:

Prof.dr. J. M. N. T. Gray,

University of Manchester

Prof.dr.ir. C. H. Venner,

Universiteit Twente

Dr. C. G. Johnson,

University of Manchester

Dr.ir. N. P. Kruyt,

Universiteit Twente

Dr. T. Weinhart,

Universiteit Twente

\section{MESA+ \\ INSTITUTE FOR NANOTECHNOLOGY \\ Nederlandse Organisatie voor Wetenschappelijk Onderzoek \\ UNIVERSITY OF TWENTE.}

The work in this thesis was carried out at the Multiscale Mechanics (MSM) group, MESA+ Institute of Nanotechnology, Faculty of Engineering Technology (ET), University of Twente, Enschede, The Netherlands.

This work was financially supported by TTW grant TTW-VICI Project10828 and TTW grant TTW-VIDI Project13472

Cover design: Marnix Pieter van Schrojenstein Lantman

Copyright (C) 2019 by M.P. van Schrojestein Lantman

Printed by: Gildeprint - Enschede

ISBN: 978-90-365-4762-8

DOI number: 10.3990/1.9789036547628

Official URL: https://doi.org/10.3990/1.9789036547628. 


\title{
A STUDY ON
}

\section{Fundamental Segregation Mechanisms \\ in Dense Granular Flows}

\section{DISSERTATION}

\author{
to obtain \\ the degree of doctor at the University of Twente, \\ on the authority of the rector magnificus, \\ Prof.dr. T.T.M. Palstra, \\ on account of the decision of the graduation committee, \\ to be publicly defended \\ on Thursday 25th April 2019 at 16:45 hrs \\ by
Marnix Pieter van Schrojenstein Lantman born on the 18th July 1988 in Velsen, the Netherlands.


This dissertation was approved by the promotor:

Dr. A. R. Thornton

and the co-promotor:

Prof.dr.rer.-nat. S. Luding 


\section{Contents}

Summary $\quad$ ix

Samenvatting $\quad$ xi

1 Introduction 1

1.1 Granular materials . . . . . . . . . . . . . . . . . . . . 1

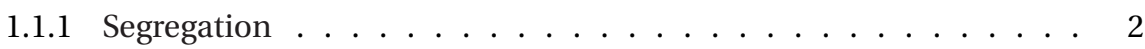

1.1.2 Research methods . . . . . . . . . . . . . . . . . . . . 3

1.2 Goals and questions . . . . . . . . . . . . . . . . . . . . 4

1.3 Dissertation overview . . . . . . . . . . . . . . . . . . 6

References...................... 6

2 A Granular Saffman Effect? 9

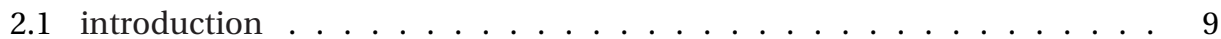

2.2 Methods . . . . . . . . . . . . . . . . . . . 11

2.3 Results ........................ 12

2.3.1 Velocity Lag . . . . . . . . . . . . . . . . . . . . . . 12

2.3.2 Pressure ...................... . . 13

2.3.3 Granular Buoyancy and Lift Force . . . . . . . . . . . . . . . . . . 14

2.3.4 Saffman Lift Force . . . . . . . . . . . . . . . . . . . . . . . . . . 15

2.3.5 Granular Saffman Lift Force . . . . . . . . . . . . . . . . . . . 16

2.4 Conclusions. . . . . . . . . . . . . . . . . . 17

References ....................... 18

3 The Granular Buoyancy Force $\quad 23$

3.1 Introduction . . . . . . . . . . . . . . . . . 23

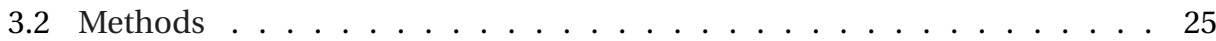

3.2.1 Simulation setup. . . . . . . . . . . . . . . . . . . 25

3.2.2 Structural analysis . . . . . . . . . . . . . . . . . . . 25

3.2.3 Coarse graining . . . . . . . . . . . . . . . . . . . . 27

3.3 Simulations. . . . . . . . . . . . . . . . . . 27

3.4 Theory . . . . . . . . . . . . . . . . . . 27

3.4.1 Buoyancy force. . . . . . . . . . . . . . . . . . . . . 29

3.4 Voronoi approach . . . . . . . . . . . . . . . . 30

3.4.3 Surface Contact density approach . . . . . . . . . . . . . . . . . 31

3.5 Results . . . . . . . . . . . . . . . . . . 34

3.5.1 Angular distribution functions . . . . . . . . . . . . . . . 34

3.5.2 Validation .................... 36

3.6 Conclusion \& Discussion . . . . . . . . . . . . . . . . . . . . . . . . . . 38

References ....................... 39 
4 Continuum Fields Around a Spherical Intruder $\quad 43$

4.1 Introduction . . . . . . . . . . . . . . . . 43

4.2 Simulation and Methods . . . . . . . . . . . . . . . . . . . . 44

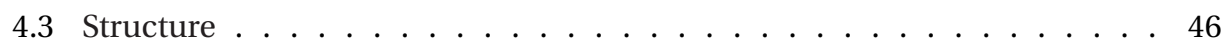

4.3.1 Density field . . . . . . . . . . . . . . . . . . . . . 47

4.3.2 Averaged layer density . . . . . . . . . . . . . . . . . 50

4.3.3 Coordination number . . . . . . . . . . . . . . . . 52

4.4 Velocity. . . . . . . . . . . . . . . . . . . . . . . . . . . . . 53

4.4.1 Fluid velocity profiles . . . . . . . . . . . . . . . . . . 55

4.4 Lag velocity . . . . . . . . . . . . . . . . . . 57

4.4 .3 Angular velocity . . . . . . . . . . . . . . . 58

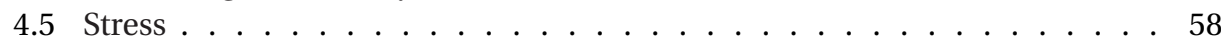

4.5.1 Pressure . . . . . . . . . . . . . . . . 60

4.5.2 Shear stress . . . . . . . . . . . . . . . . . . . 62

4.5.3 Bulk friction . . . . . . . . . . . . . . . . . . 62

4.5.4 Out-of-plane anisotropy . . . . . . . . . . . . . . . . 65

4.6 Conclusions \& discussions . . . . . . . . . . . . . . . . . . . . . . . 65

References...................... . . 67

5 Force Model for a Large Intruder $\quad 71$

5.1 Introduction . . . . . . . . . . . . . . . . . 71

5.2 Methods and simulations . . . . . . . . . . . . . . . . . 72

5.3 Results ....................... . . 73

5.3.1 Segregation direction . . . . . . . . . . . . . . . . 75

5.3.2 Segregation mechanism . . . . . . . . . . . . . . . 76

5.4 Force model. . . . . . . . . . . . . . . . . . . . 79

5.4.1 Reference forces . . . . . . . . . . . . . . . . . 80

5.4 Lift forces . . . . . . . . . . . . . . . . . . . . . 81

5.4 .3 Drag forces. . . . . . . . . . . . . . . . 82

5.4 .4 Force model . . . . . . . . . . . . . . . . . . . 82

5.5 Conclusions and discussions . . . . . . . . . . . . . . . . . . . . . . . . 83

References . . . . . . . . . . . . . . . . . . 85

6 Continuum Simulations of Granular Media $\quad 89$

6.1 Introduction . . . . . . . . . . . . . . . . . . . 89

6.2 Simulation Model . . . . . . . . . . . . . . . . . . . 91

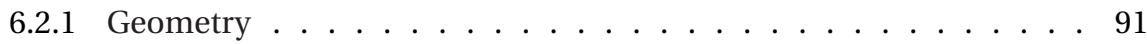

6.2.2 Governing Equations. . . . . . . . . . . . . . . . . . 93

6.2.3 Rheology. . . . . . . . . . . . . . . . . . . 94

6.3 Methods ........................... 95

6.3.1 Solution method . . . . . . . . . . . . . . . . 96

6.3 .2 regularisation . . . . . . . . . . . . . . 96

6.3.3 Benchmark .................... . . 97

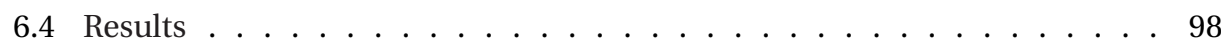

6.5 conclusion \& discussion . . . . . . . . . . . . . . . . 101

References . . . . . . . . . . . . . . . . . 103 
7 Conclusions and Outlook 107 References ....................... 110

A A Granular Saffman Effect: Supplementary Material 111

A.1 Horizontal force balance and velocity lag . . . . . . . . . . . . . . . . 111

A.2 Inclination angle dependence of the lift force . . . . . . . . . . . . . . . 113

A.3 Depth dependence of the lift force . . . . . . . . . . . . . . . . . 113

References . . . . . . . . . . . . . . . . . . 113

B Force Model for a Large Intruder: Supplementary Material 115

B.1 Flow gradients . . . . . . . . . . . . . . . . . . 115

References ........................ . . 119

C Parallel Particle Simulations in MercuryDPM 121

C.1 Introduction . . . . . . . . . . . . . . . . . . . 121

C.1.1 Parallel methods . . . . . . . . . . . . . . . . . . . . 122

C.1.2 MercuryDPM simulation method . . . . . . . . . . . . . . . 123

C.2 Parallel Algorithm. . . . . . . . . . . . . . . . . . . . . . . . . . . . . 124

C.2.1 Local communication structure . . . . . . . . . . . . . . . . . . 124

C.2.2 Periodic boundary communication structure . . . . . . . . . . . . 129

C.2.3 Insertion and Deletion . . . . . . . . . . . . . . . . . . . . . . . . 132

C.2.4 Parallel algorithm implementation. . . . . . . . . . . . . . . 133

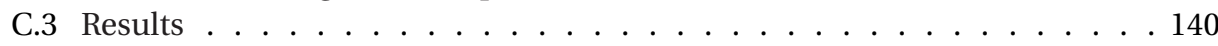

C.4 Conclusion and discussion . . . . . . . . . . . . . . . . . . . 140

References....................... 142

$\begin{array}{ll}\text { Acknowledgements } & 145\end{array}$

$\begin{array}{ll}\text { Curriculum Vitae } & 147\end{array}$ 



\section{SUMMARY}

Segregation in dense granuar flows occurs due to particles having different properties, with particle size and density playing a dominant role among others such as shape and surface roughness. A good understanding of segregation of realistic materials is required to avoid costly unnecessarily long or re-mixing operations in industrial plants and to predict the evolution of natural hazards like avalanches and pyroclastic flows.

Segregation in sheared granular flows is normally described in terms of kinetic sieving, where the larger particles act as a sieve for smaller particles, and squeeze expulsion, where larger particles are squeezed out of their layer in the opposite direction of the smaller particles. The aim of this research is to better understand the micro-mechanical origins of segregation by numerical simulations and to develop models that can qualitatively predict segregation. The considered system is a monodisperse flow with a single large intruder, effectively removing kinetic sieving, but keeping squeeze expulsion.

First, the analogy to a single particle in a standard Newtonian fluid is taken by considering a model of buoyancy, drag and lift forces. Two remarkable discoveries are: (i) an upstream velocity is measured which is correlated to the lift force and (ii) the granular buoyancy force being different to Archimedes' law. Further investigations into the buoyancy force show that the difference stems from a lack of scale separation between the bulk particles and the intruder. For increasing intruder size, the number of contacts per intruder surface area reduces, effectively reducing the buoyancy force. This contact mechanism is captured accurately by a Voronoi volume correction to Archimedes' law.

The second approach is to visualise the mechanisms of segregation by analysing how the intruder size, density and friction affects the granular flow. This is done by converting the discrete particle simulation data into smooth conservative continuum (density, velocity, stress) fields with a technique called coarse graining. These fields show that a large intruder does not fit inside a layer of bulk particles leading to an anisotropic stress field. This observation has inspired new scalings for the lift force on an intruder, proportional to the shear rate and viscosity gradient of the bulk flow. Simulations for many different flows have been performed to confirm this hypothesis.

The segregation strength of an intruder depends on the granular flow. Hence, simulations of granular flows with continuum methods are performed. A generalised $\mu(I)-$ rheology in a split-bottom shear cell setup has been simulated, with a new correction for low inertial values. Results show improvement compared to the classical $\mu(I)$-rheology, however further corrections are recommended.

The fundamental mechanisms discovered in this thesis have improved the understanding of individual particles in granular flows, which can be used to develop more accurate continuum models for segregation. The developed micro-based force model can be used as starting point to develop more sophisticated models that could aid in the engineering of granular materials by balancing size with density and other realistic particle properties with the goal of reducing segregation. 



\section{SAMENVATTING}

Segregatie in granulaire stromingen met hoge dichtheid vindt plaats als gevolg van de verschillende eigenschappen van de granulaire deeltjes. Hierbij spelen de grootte en dichtheid een dominante rol, maar ook o.a. de vorm en oppervlakteruwheid hebben invloed. Een goed begrip van segregatie is belangrijk in de industrie om het onnodig of opnieuw mengen van granulaire materialen te voorkomen, ter reductie van kosten en energie. Verder speelt het een belangrijke rol bij het voorspellen van natuurlijke gevaren zoals lawines en pyroclastische stromen.

Normaal wordt segregatie in dichte, granulaire stroming beschreven door kinetic sieving, waarbij de grotere deeltjes fungeren als een zeef voor kleinere deeltjes, en squeeze explusion, waarbij grotere deeltjes uit hun laag worden gedrukt, in tegenovergestelde richting van de kleinere deeltjes. Het doel van dit onderzoek is om de micromechanische oorsprong van segregatie beter te begrijpen met behulp van numerieke simulaties en om een model te ontwikkelen dat segregatie kwalitatief kan voorspellen. Het bestudeerde systeem bestaat uit identieke deeltjes met een enkel groter deeltje. Hierdoor wordt effectief kinetic sieving verwijderd, maar wordt squeeze expulsion behouden.

In een eerste benadering wordt de analogie genomen met een deeltje in een standaard Newtoniaanse vloeistof door een model te beschouwen met een drijf-, weerstanden liftkracht. Twee opmerkelijke ontdekkingen zijn: (i) een stroomopwaartse snelheid wordt gemeten die verband houdt met de liftkracht en (ii) de drijfkracht verschilt met de wet van Archimedes. Nader onderzoek naar de drijfkracht toont aan dat het verschil komt doordat de omvang van de stromingsdeeltjes en het grote deeltje van gelijke schaal is. Het aantal contacten per oppervlak van het grote deeltje neemt af naarmate het grote deeltje groter wordt, hierdoor vermindert de drijfkracht. Dit mechanisme kan nauwkeurig worden beschreven door een Voronoi-volumecorrectie toe te passen op de wet van Archimedes.

De tweede benadering is om segregatie zichtbaar te maken door te visualiseren hoe de grootte, dichtheid en wrijving van het grote deeltje de granulaire stroom beïnvloedt. Dit wordt gedaan door de discrete simulatiedata van deeltjes om te zetten in conservatieve continuumvelden (dichtheid, snelheid, spanning) met een techniek die coarse graining wordt genoemd. Deze velden laten zien dat een groot deeltje niet in een laag van stromingsdeeltjes past, waardoor een anisotroop spanningsveld ontstaat. Deze waarneming heeft tot een nieuwe schaling geleid voor de liftkracht op het grote deeltje, welke evenredig is met de afschuifsnelheid en de gradient inviscositeit van de granulaire stroming. Simulaties voor veel verschillende stromen zijn geanalyseerd om deze hypothese te bevestigen.

De sterkte van segregatie van een groot deeltje hangt af van de granulaire stroming. Vandaar dat simulaties van granulaire stromingen met continuummethoden worden uitgevoerd. Een gegeneraliseerde $\mu(I)$-reologie is gesimuleerd in een ronde afschuifop- 
stelling met een gespleten bodem. De resultaten laten een verbetering zien in vergelijking met de klassieke $\mu(I)$-reologie, maar verdere correcties worden aanbevolen.

De nieuw ontdekte mechanismen die in dit proefschrift zijn beschreven, hebben het begrip van individuele deeltjes in granulaire stromingen verbeterd. Deze inzichten kunnen worden gebruikt om meer accurate continuummodellen voor segregatie te ontwikkelen. Verder kan het ontwikkelde krachtenmodel worden gebruikt als uitgangspunt voor het ontwikkelen van meer geavanceerde modellen. Deze modellen kunnen helpen bij het ontwerpen van granulaire materialen met gereduceerde segregatie, door de grootte van deeltjes te balanceren met dichtheid en andere realistische eigenschappen. 


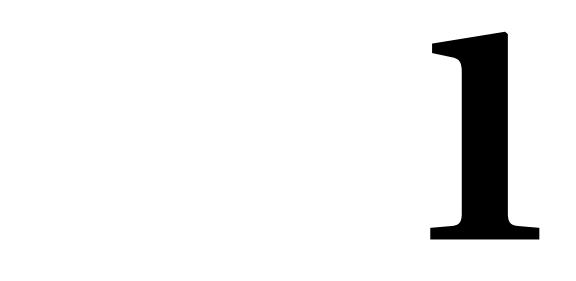

\section{INTRODUCTION}

\subsection{GRANULAR MATERIALS}

Granular materials can be defined as a collection of discrete particles dissipatively interacting with each other. This is a very general definition and therefore many examples of granular materials exist such as coffee powder, snow, coal, sand and even the asteroid belts in the solar system.

When undisturbed, granular materials show solid-like behaviour (e.g. a pile of sand). However, when agitated, the material exhibits fluid-like behaviour. The individual particles start to rearrange themselves in a flow-like movement, commonly referred to as a granular flow. These flows can be categorised into two types, a granular liquid and granular gas. In granular liquids the individual particles are packed closely together and they are therefore commonly referred to as dense granular flows. An example of such flow can be observed when pouring coffee powder. An example of a granular gas is a dust storm, where there is quite some distance between the particles.

The properties of the individual particles ultimately determine how the granular material behaves on a large (industrial) scale. The individual particles in a granular material can be described by properties such as size, shape, density or surface roughness, amongst others. Often granular materials consist of distinctly different particles. As an example see Fig. 1.1(a), where the granular material consists of two different sizes of particles: large pebbles and small sand grains.

Dense granular flows of such bidisperse materials show a peculiar phenomenon called segregation. An experimental observation of segregation is shown in Fig. 1.1(b) [1]. Here an initially homogeneously mixed material flows down an incline. The mixture consists of two differently sized particle types: large particles (red) and small particles (white). As these particles flow down the incline they segregate into a large particle phase on top and a small particle phase on the bottom. In this particular case segregation occurs due to a difference in size, however a difference in density or other properties can induce segregation too.

Granular materials are widely used in many industries such as the agricultural, pharmaceutical, food and mining industry. Knowing how to efficiently handle granular ma- 

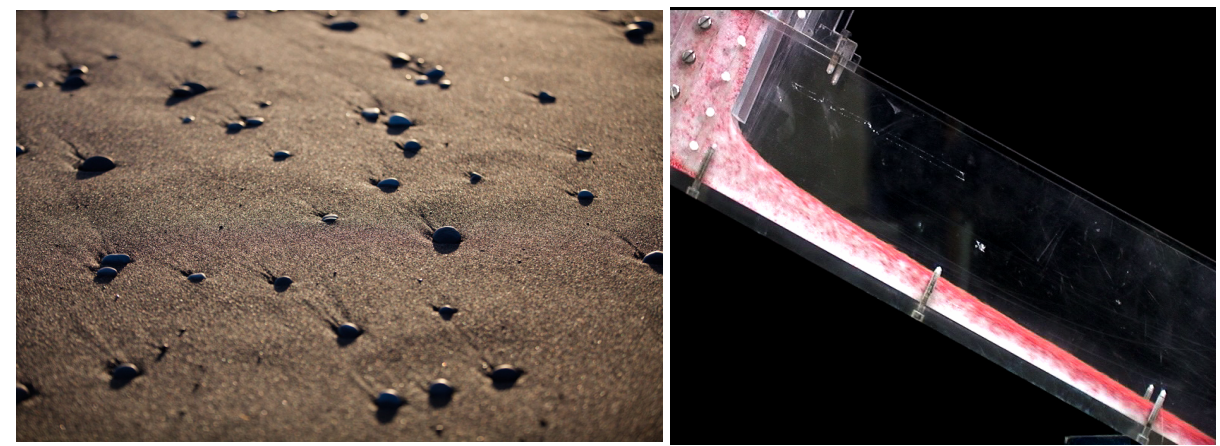

Figure 1.1: a) A typical granular material found in nature, containing dissimilar grains. This specific example consist of fine sand and small stones. b) An experimental setup of a granular material flowing down an incline. At the top-left the material is homogeneously mixed, containing large (red) and small (white) particles. The material starts flowing due to gravity and segregates into a large particle phase on top and a small particle phase at the base. The image on the right is reproduced from [1].

terials is therefore important if one aims at reducing operation costs and energy consumption.

An important goal in industry is to create homogeneous mixtures of different types of granular materials, such as spice mixes in the food industry, or medicinal powder in the pharmaceutical industry. Homogeneous mixtures could be created by agitating the granular material such that it starts to flow, rearranging the individual particle positions in a process generally called mixing, however, segregation counteracts this. When a material is homogeneously mixed, it is important to keep it mixed during transport. However, during transport often segregation occurs and costly re-mixing operations are frequently required. A thorough understanding of this phenomenon could lead to a reduction of segregation in granular materials, yielding more efficient industrial plants.

\subsubsection{SEgREgATION}

A number of segregation mechanisms have been proposed for dense sheared granular media [2, 3]. One important mechanism is the percolation of small particles, driven by gravity. Two regimes can be identified, spontaneous percolation [4] and kinetic sieving as defined in [5]. Spontaneous percolation occurs when a particle is so small that it can fall through the matrix of large particles without resistance. Kinetic sieving requires a sheared flow for the small particle to percolate; the flow dilates due to shear, creating space for particles to percolate. Statistically, small particles have a higher chance to percolate compared to large particles. Another segregation mechanism is squeeze expulsion, where a particle is pushed out of its current layer [5]. The combination of kinetic sieving and squeeze expulsion is termed gravity-driven segregation [3,6]. A different description is based on the granular temperature $[7,8]$. Small and large particles are attracted to regions of low granular temperature. However, small particles are typically more kinetic and arrive first in these regions [9]. An alternative segregation description has been given by extending kinetic theory to dense granular flows [10, 11]. 
Although these mechanisms give phenomenological explanations of why segregation occurs, quantifying segregation remains a challenge. In order to possibly unify all these theories it is important - as a starting point - to understand the microscopic details of the extreme limit case of a single particle in dense granular flows.

This has led to research on the drag and lift forces on single particles in dense granular flows. The lift forces on a single intruder particle of varying size in a $2 \mathrm{D}$ shear flow have been investigated in Ref. [12]. To refrain the particle from moving away it was attached to a spring. The authors proposed two competing segregation mechanisms that depend on the gradient of shear stress and the gradient of the pressure, respectively. Investigation of a drag force due to density segregation in a 3D chute flow was performed in Ref. [13]. The observed drag force is similar to the Stokesian drag force measured in classical fluids, although the drag coefficient changes with the chute angle [14]. These and other investigations [15-18] highlight significant details of the fundamentals of segregation, but a complete description is still missing.

\subsubsection{RESEARCH METHODS}

Experimental investigations into the fundamental mechanisms of segregation are hampered by two important limitations. Firstly, most granular materials are opaque and therefore it is not possible to look inside the material, even if the individual particles are transparent. Standard cameras are therefore limited to tracking the particles at transparent walls or surfaces (e.g. see Fig. 1.1(b)).

Experimental techniques have been developed that can overcome this limitation. Refractive Index-Matched Scanning (RIMS) is a technique that can accurately capture the internal structure of transparent granular materials [19]. Particle position and velocity can be measured in a 2D intersection of the experiment, while only the position can be measured when investigating a volume due to slow scanning time [20]. Positron emission particle tracking (PEPT) is a technique that radioactively labels a single particle [21], enabling high resolution position and velocity tracking. Only recently an X-ray technique has been developed that is capable of determining the flow profile of rapidly flowing steady state systems and potentially transient systems [22].

A second experimental limitation is the lack of measurement of inter-particle forces. One method to observe the stresses between particles is by using particles of photoelastic material [23]. This material changes optical properties when elastically deformed, enabling a visual measurement of stress. Thus far this method can only be applied to 2D systems and no success in 3D has been reported.

Segregation in granular material is an interplay between particle velocities and contact forces, and therefore both need to be studied simultaneously. Measuring the combination of both contact forces and flow profile in an experiment is very challenging, if not impossible. As an alternative, computer simulations can be used to investigate segregation, since discrete simulation methods track the position, velocity and forces of each individual particle.

There are a number of discrete simulation methods used for granular matter. EventDriven simulations solve the motions of particles and predict when collisions occur. The collisions between particles are solved instantaneously and time is forwarded to the next collisional event [24]. The method is well-suited for dilute to moderately dense flows 
and hard spheres. Another method is the non-smooth Contact Dynamics method [25] which is based on two conditions, (i) particles can not penetrate each other and (ii) a discontinuous Coulomb friction law describes the tangential forces. An alternative is the Discrete Particle Method (DPM) [26] which relaxes the non-penetration and discontinous Coulomb constraints, allowing overlaps. These overlaps are then related to contact forces through contact laws. The relaxation of the non-penetration constraint enables extra freedom allowing many different (complex) contact laws and is relatively easy to implement. It is therefore a widely used simulation method for granular materials.

In DPM, the motion of every particle $p$ is described by Newton's second law,

$$
\boldsymbol{F}_{p}=m_{p} \boldsymbol{a}_{p}
$$

where $\boldsymbol{F}_{p}$ is the total force on particle $p, m_{p}$ is the mass of the particle and $\boldsymbol{a}_{p}$ is the acceleration. Numerical integration of the acceleration yields the velocity and positions of particles. The forces on the particle consists of body forces such as the gravity force, but also on the contact forces between neighbouring particles. These contact forces are modelled by contact laws and are crucially important in a DPM simulation. The challenge is to adopt a contact law that captures most of the contact physics, yet remains simple for fast computations.

Often the contact force is decomposed in a normal and a tangential contact component. For dry, non-cohesive materials a physically accurate contact law takes the contact theory of Hertz [27] for the forces in the normal direction and the theory of Mindlin [28] for forces in the tangential direction, also known as the Hertz-Mindlin contact model. This law has a non-linear dependence on the overlap between particles with a history dependent tangential contact stiffness. A more simple and computationally less demanding contact law is the linear viscoelastic contact law developed originally in Ref. [26]. The normal contact forces are modeled by a spring-dashpot system. Here the elastic deformation of the contact is modeled by a linear elastic spring and the dashpot introduces dissipation proportional to the velocity. The tangential force model is similar to the normal contact force model, with the addition of a slider allowing Coulomb friction. Comparison between the Hertz-Mindlin contact law and the simplified linear contact law shows that the latter is surprisingly accurate for granular flows [29, 30]. As a result of the simplicity and accuracy of the linear contact law, it is the most commonly used contact law in DPM simulations. More complicated contact laws have introduced dry particle cohesion [31] and liquid bridges [32], see also references therein, but are not subject of this thesis.

Due to its simplicity, DPM methods are widely used in granular research. It is used to investigate granular phenomena in more detail than experiments and to develop models that predict the behaviour of granular materials. It is important to stress that these models should always be validated by experiments, using realistic granular materials.

\subsection{GOALS AND QUESTIONS}

The aim of this research is to fundamentally understand segregation of a single intruding particle with different size, density and friction, compared to the granular flow particles. This is done by developing a force model that captures its behaviour. The behaviour 
of the intruder is studied using DPM simulations and the force model to describe this behaviour is based on analogies with a particle in a normal fluid. The first four questions are related to testing these analogies, while the fifth question is related to continuum models for the base-granular flow without intruder.

- Q1 Does the intruder experience a lag velocity in flow-direction and is it related to a lift force on the intruder?

A correlation between a particle lag and a lift force is sought by drawing an analogy with lift forces observed in classical fluid dynamics. By performing DPM simulations in a steady state flow down an incline, long time-averaged force and velocity statistics are obtained. The intruder particle is attached to a virtual spring to prevent it from moving upward and enabling lift force measurements.

- Q2 What is the buoyancy force on an intruder particle?

The classical buoyancy force on an intruder in a fluid is proportional to the fluid volume displaced by the intruder. A Voronoi volume approach allows for a geometrical interpretation of the displaced volume, but only if the intruder and bulk particle size are identical. For larger intruders it is not clear how to define the displaced volume due to the void spaces between particles. By analysing the force distribution and contact structure around the intruder a more complete theory is developed and validated.

- Q3 What is the effect of the intruder size, density and friction on the granular flow?

As the intruder changes properties, the flow does not only have an effect the intruder, but the intruder also affects the flow. Visualising this is done by converting discrete particle data into continuum fields, yielding high resolution density, velocity and stress fields around the intruder. These fields shed new light on the various segregation mechanisms.

- Q4 Can the segregation force on an intruder be captured by a force model?

Answering this research question is done by combining all observations and insights from Q1, Q2 and Q3 and validating this for different granular flow situations.

- Q5 Can a continuum model using a generalised $\mu(I)$ - rheology simulate granular materials in a split-bottom ring shear cell?

The segregation strength of an intruder depends on the granular flow around the intruder. Obtaining such granular flows efficiently could be done with continuum simulation methods, if the correct rheology is known and used. Here a newly developed generalised $\mu(I)$-rheology [33] is investigated in a split-bottom ring shear cell to investigate the base flow without intruder. 


\subsection{DisSERTATION OVERVIEW}

The outline of this thesis follows the research questions. In Chapter $2 \mathbf{Q} \mathbf{1}$ is addressed and answered, resulting in a preliminary force model of the intruder particle. Two important aspects of this model are a buoyancy force model and a lift force model. The buoyancy force depends on size and is further investigated in Chapter 3, answering question Q2. In Chapter 4 the effect of the intruder on the granular flow is visualised, answering Q3 and in Chapter 5 the force model is finalised by uncovering a general segregation mechanism for the lift force, answering Q4. In Chapter 6 a recently proposed granular rheology is tested in a split-bottom ring shear cell, addressing Q5. Conclusions and outlook of this thesis are discussed in Chapter 7. Additionally, in Appendix C an algorithm is presented for highly parallelised DPM simulations.

\section{REFERENCES}

[1] A. R. Thornton, A Study of Segregation in Granular Gravity Driven Free Surface Flows, Ph.D. thesis, The University of Manchester (2005).

[2] J. J. McCarthy, Turning the corner in segregation, Powder Technology 192, 137 (2009).

[3] J. M. N. T. Gray, Particle segregation in dense granular flows, Annual Review of Fluid Mechanics 50, 407 (2018).

[4] A. M. Scott and J. Bridgwater, Interparticle percolation: A fundamental solids mixing mechanism, Industrial \& Engineering Chemistry Fundamentals 14, 22 (1975).

[5] S. Savage and C. Lun, Particle size segregation in inclined chute flow of dry cohesionless granular solids, Journal of Fluid Mechanics 189, 311 (1988).

[6] J. M. N. T. Gray and A. R. Thornton, A theory for particle size segregation in shallow granular free-surface flows, in Proceedings of the Royal Society of London A: Mathematical, Physical and Engineering Sciences, Vol. 461 (The Royal Society, 2005) pp. 1447-1473.

[7] Y. Fan and K. M. Hill, Phase transitions in shear-induced segregation of granular materials, Physical Review Letters 106, 218301 (2011).

[8] Y. Fan and K. M. Hill, Theory for shear-induced segregation of dense granular mixtures, New Journal of Physics 13, 095009 (2011).

[9] D. R. Tunuguntla, T. Weinhart, and A. R. Thornton, Comparing and contrasting sizebased particle segregation models, Computational Particle Mechanics 4, 387 (2017).

[10] M. Larcher and J. T. Jenkins, The evolution of segregation in dense inclined flows of binary mixtures of spheres, Journal of Fluid Mechanics 782, 405-429 (2015).

[11] M. Larcher and J. T. Jenkins, Segregation and mixture profiles in dense, inclined flows of two types of spheres, Physics of Fluids 25, 113301 (2013). 
[12] F. Guillard, Y. Forterre, and O. Pouliquen, Scaling laws for segregation forces in dense sheared granular flows, Journal of Fluid Mechanics 807 (2016).

[13] A. Tripathi and D. V. Khakhar, Numerical simulation of the sedimentation of a sphere in a sheared granular fluid: a granular stokes experiment, Physical Review Letters 107, 108001 (2011).

[14] A. Tripathi and D. V. Khakhar, Density difference-driven segregation in a dense granular flow, Journal of Fluid Mechanics 717, 643-669 (2013).

[15] S. Liu and J. J. McCarthy, Transport analogy for segregation and granular rheology, Physical Review E 96, 020901 (2017).

[16] L. Staron, Rising dynamics and lift effect in dense segregating granular flows, Physics of Fluids 30, 123303 (2018).

[17] L. Jing, C. Kwok, and Y. Leung, Micromechanical origin of particle size segregation, Physical Review Letters 118, 118001 (2017).

[18] N. Thomas and U. D'ortona, Evidence of reverse and intermediate size segregation in dry granular flows down a rough incline, Physical Review E 97, 022903 (2018).

[19] J. A. Dijksman, F. Rietz, K. A. Lőrincz, M. van Hecke, and W. Losert, Invited article: Refractive index matched scanning of dense granular materials, Review of Scientific Instruments 83, 011301 (2012).

[20] K. van der Vaart, A. Thornton, C. Johnson, T. Weinhart, L. Jing, P. Gajjar, J. Gray, and C. Ancey, Breaking size-segregation waves and mobility feedback in dense granular avalanches, Granular matter 20, 46 (2018).

[21] D. Parker, R. Forster, P. Fowles, and P. Takhar, Positron emission particle tracking using the new Birmingham positron camera, Nuclear Instruments and Methods in Physics Research Section A: Accelerators, Spectrometers, Detectors and Associated Equipment 477, 540 (2002).

[22] J. Baker, F. Guillard, B. Marks, and I. Einav, X-ray rheography uncovers planar granular flows despite non-planar walls, Nature Communications 9, 5119 (2018).

[23] T. S. Majmudar and R. P. Behringer, Contact force measurements and stress-induced anisotropy in granular materials, Nature 435, 1079 (2005).

[24] M. Alam and S. Luding, Rheology of bidisperse granular mixtures via event-driven simulations, Journal of Fluid Mechanics 476, 69 (2003).

[25] M. Jean, The non-smooth contact dynamics method, Computer Methods in Applied Mechanics and Engineering 177, 235 (1999).

[26] P. A. Cundall and O. D. Strack, A discrete numerical model for granular assemblies, Geotechnique 29, 47 (1979). 
[27] H. R. Hertz, Über die Berührung fester elastischer Körper und über die Härte, Verhandlung des Vereins zur Beforderung des Gewerbefleißes, Berlin , 449 (1882).

[28] R. D. Mindlin, Elastic spheres in contact under varying oblique forces, Journal of Applied Mechanics 20, 327 (1953).

[29] A. Di Renzo and F. P. Di Maio, Comparison of contact-force models for the simulation of collisions in dem-based granular flow codes, Chemical Engineering Science 59, 525 (2004).

[30] A. R. Thornton, T. Weinhart, S. Luding, and O. Bokhove, Frictional dependence of shallow-granular flows from discrete particle simulations, The European Physical Journal E 35, 127 (2012).

[31] S. Luding, Cohesive, frictional powders: contact models for tension, Granular matter 10, 235 (2008).

[32] S. Roy, A. Singh, S. Luding, and T. Weinhart, Micro-macro transition and simplified contact models for wet granular materials, Computational Particle Mechanics 3, 449 (2016).

[33] S. Roy, S. Luding, and T. Weinhart, A general(ized) local rheology for wet granular materials, New Journal of Physics 19, 043014 (2017). 


\title{
2
}

\section{SEgREgATION OF LARGE PARTICLES}

\author{
IN DENSE GRANULAR FLOWS \\ SUGGESTS A GRANULAR SAFFMAN \\ EFFECT
}

We report on the scaling between the lift force and the velocity lag experienced by a single particle of different size in a monodisperse dense granular chute flow. The similarity of this scaling to the Saffman lift force in (micro) fluids, suggests an inertial origin for the lift force responsible for segregation of (isolated, large) intruders in dense granular flows. We also observe an anisotropic pressure field surrounding the particle, which potentially lies at the origin of the velocity lag. These findings are relevant for modelling and theoretical predictions of particle-size segregation. At the same time, the suggested interplay between polydispersity and inertial effects in dense granular flows with stress- and straingradients, implies striking new parallels between fluids, suspensions and granular flows with wide application perspectives.

\subsection{INTRODUCTION}

Size-polydispersity is intrinsic to non-equilibrium systems like granular materials [2]. It gives them the ability to size-segregate when agitated, a process which spatially separates different sized grains [3-8], but is different from phase separation in classical fluids. Particle-size segregation in dense granular flows $[9,10]$ has been intensively studied [e.g. 11-26], but a fundamental question remains unanswered: why do large particles segregate?

This chapter has been published in Physical Review Fluids 3, (2018) [1]. Kasper van der Vaart and Marnix P. van Schrojenstein Lantman have contributed equally to this study. 
It is generally understood that in dense granular flows both small and large particles are pushed away from high shear regions $[12,13]$ or pulled by gravity $[14,15]$. The reason for the separation of large and small particles is that small particles are more mobile and are therefore more effectively pulled or pushed. They can carry proportionally more of the kinetic energy [17-20], and are statistically more likely to move into gaps between larger particles. This process is referred to as kinetic sieving [14, 15]. However, when the large-particle concentration (volume fraction) is very low and there are no gaps for small particles to move in to, arguably the concept of kinetic sieving breaks down. Thus a qualitative-let alone a quantitative-understanding of size-segregation in this regime is lacking.

Current models for size-segregation in dense granular flows perform well when the small and large-particle concentrations (volume fractions) are nearly equal [13, 27-30]. When accounting for the effect of size-segregation asymmetry [31,32], models have been extended to more unequal concentrations, but they remain inaccurate in the limit of low large-particle concentrations. Extending models to this limit is critical because during segregation, and even after reaching a steady state, regions of low large-particle concentration occur and can persist throughout the flow [24, 26, 31]. Moreover, current models are either completely or partly phenomenological. Thus, to advance modelling, we should aim to understand the physical origin of size-segregation allowing us to derive the free state-variables from their microscopic quantities. An important related issue is that current constitutive models for dense granular flows only work with an average particle size [33, 34]. If we are to implement size-distributions in these models a better understanding of micro-scale effects between large and small particles seems crucial.

In contrast to particle-size segregation, particle migration in suspensions, in the limit of low concentrations, is generally well understood [e.g. 35, 36]. Arguably this progress has been aided by the fact that the fluid forces acting on a particle can be calculated, which can not be said for granular media. This inspired us to treat the particles that surround an intruder as a continuum and attempt to understand the forces acting on a segregating particle based on the measured continuum fields.

Recently, Guillard et al. [37] measured for the first time the segregation lift force on a single large intruder particle in a mono-disperse granular flow by attaching the intruder to a virtual spring perpendicular to the plane (see Fig. 2.1). They found scaling laws that linked the total upward force or net contact force on the intruder to shear and pressure gradients. These scaling laws predict the direction of segregation of large particles in different flow configurations depending on whether a shear or pressure gradient has the strongest contribution. However, they do not shed any light on the origin of the lift force.

In this study we present new physical insights into the origin of the segregation lift force on large intruders in three-dimensional mono-disperse dense granular flows. We do so, firstly, by taking a different approach to Guillard et al. [37] and determine the lift force $F_{L}$ by decomposing the net contact force on an intruder as $F_{c}=F_{L}+F_{b}$, where $F_{b}$ is a generalised buoyancy force for dense granular media that accounts for the local geometry around an intruder. This novel approach is inspired by our finding of an anisotropic pressure field that surrounds the intruder and grows with its size. Secondly, we report on a velocity lag of the intruder relative to the bulk flow and demonstrate a scaling between this velocity lag and the lift force. The similarity of this scaling to the known Saffman lift 


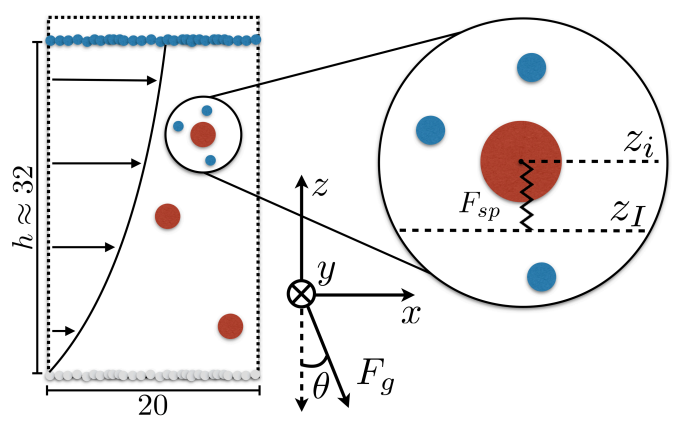

Figure 2.1: Schematic of the simulations: 3D mono-disperse granular flow down an incline, with angle $\theta=22^{\circ}$. Only base (white) and surface (blue) particles are shown, as well as three bulk particles. The flow contains three intruder particles that are held with springs around three different $z$-positions $z_{0}$ (intruder positions in the schematic are to scale), but move freely in the $x$ - $y$ plane.

force in fluids and the presence of the anisotropic pressure field, allow us to propose a physical origin for the segregation lift force.

\subsection{Methods}

We use MercuryDPM, based on discrete particle methods (MercuryDPM.org; [38, 39]), and investigate three-dimensional (3D) flows of mixtures of spherical dry frictional particles flowing down an incline of $\theta=22^{\circ}$. We verified that changing the inclination angle between $22^{\circ}$ and $26^{\circ}$ has no significant effect (within the fluctuations) on the measured lift force $F_{L}$ (see Appendix A.2). All simulation parameters are non-dimensionalised such that the particle density is $\rho_{p}=6 / \pi$ and the gravitational acceleration is $g=1$, with downward vertical component $g_{z}=-\cos \theta$. The simulations are conducted in a box with dimensions $\left(L_{x}, L_{y}, L_{z}\right)=(20,8.9, \infty)$, with periodic walls in the $x$ - and $y$-direction. The particles that make up the bulk of the flow have a diameter $d_{b}=1$. We vary the intruder diameter $d_{p}$ between size ratios $S=d_{p} / d_{b}=0.5$ and 3.2. The rough base of the chute consists of particles of radius 0.85 and the flow height is $h=32 \pm 0.5$.

A linear spring-dashpot model $[40,41]$ with linear elastic and linear dissipative contributions is used for the normal forces between particles. The restitution coefficient for collisions is chosen $e_{r}=0.1$ and the contact duration is $t_{c}=0.005$. This results in a different stiffness depending on the particle size. We verified that our findings are not the result of this difference in stiffness nor the dependence on $e_{r}$ and $t_{c}$. The friction coefficient for contacts between bulk particles $\mu_{b b}$ and between bulk and intruder particles $\mu_{b p}$ equals 0.5 , unless otherwise stated.

We place three identical intruders in the flow at vertical positions $z_{p, 0}=5,15$ and 23 (see Fig. 2.1). Each intruder is attached to a spring [37], which applies a vertical force $F_{s p}=-k\left(z_{p}-z_{p, 0}\right)$ proportional to the vertical distance between the intruder position $z_{p}$ and its corresponding $z_{p, 0}$. Here $k_{s}=20$ is the spring stiffness. We also simulate $k_{s}=\infty$ by fixing the intruder at $z_{p}=z_{p, 0}$. Our findings are independent of $k_{s}$, so unless stated otherwise all data reported are for $k_{s}=20$. We do not discuss the data for $z_{p, 0}=5$ 
because the intruder experiences boundary effects, likely due to layering near the bed, as reported in [41].

The net contact force $F_{c}$ on an intruder can be determined in two ways: (i) Through the force balance $-F_{c}+F_{s p}-F_{g_{z}}=0$, where $F_{s p}$ is computed from the intruder's average vertical position, and $F_{g_{z}}=\rho_{p} g_{z} V_{p}$ is the positively defined gravity force, with $V_{p}=\frac{4}{3} \pi\left(d_{p} / 2\right)^{3}$ the intruder volume; (ii) By using the force balance $F_{c}=F_{n_{z}}+F_{t_{z}}$, with $F_{n_{z}}$ and $F_{t_{z}}$ the vertical normal and tangential contact forces, respectively. We verified that both methods give the same answer.

Applying coarse-graining (CG) [41-43], after a steady state has been reached, we obtain time-averaged 3D continuum fields for $\phi$ the local solids fraction, and $\sigma$ the stress tensor, which satisfy the conservation laws. From the stress tensor we calculate the pressure field $p=\operatorname{Tr}(\boldsymbol{\sigma}) / 3$ and the shear stress field $\boldsymbol{\tau}=\boldsymbol{\sigma}-p \boldsymbol{I}$. The CG-width is chosen of the order of the particle diameter $w=d_{b}$ to achieve both rather smooth fields and independence of the fields on $w$ [42]. We approximate the bulk solids fraction at the position of the intruder $\phi\left(x_{p}, y_{p}, z_{p}\right)=\phi_{p}=V_{p} / V_{V, p}$ using the ratio of the particle volume $V_{p}$ and the Voronoi volume $V_{V, p}$, which we obtain through $3 \mathrm{D}$ raidus-weighted Voronoi tessellation (math.lbl.gov/voro++; [44]). All error-bars (shaded areas) correspond to a $95 \%$ confidence interval.

\subsection{RESULTS}

\subsubsection{VELOCITY LAG}

Our first and most obvious finding is that intruders that have a size ratio larger than one $(S>1)$ are positioned (on average) above $z_{p, 0}$, thus with a non-zero and negative value of $F_{s p}$. Our second finding is that the downstream velocity $v_{x i}$ of an intruder with $S>1$, experiences a lag $\lambda_{x}=\left\langle u_{p, x}(t)-u_{x}\left(z_{p}, t\right)\right\rangle$ with respect to the downstream velocity $u_{x}\left(z_{p}\right)$ of the bulk at height $z_{p}$, where $\langle\ldots\rangle$ corresponds to a time average. Figure 2.2(a) shows that a large intruder $(S>1)$ lags $\left(\lambda_{x}<0\right)$, while a same sized intruder $(S=1)$ experience no lag, within the fluctuations. Interestingly, but outside the scope of this study, for $S<1$, when the intruder is smaller than the bulk particles and sinks, $\lambda_{x}$ flips sign and becomes a velocity raise (increase). Figure $2.2(b)$ shows that the lag velocity increases at higher positions in the flow.

Based on the derivation in Appendix A.1 we propose the following expression for the lag:

$$
\lambda_{x}=\frac{1}{\pi d_{b}} \frac{1}{\eta} \frac{\Delta F(S)}{c(S) S}
$$

where $c(S)$ is a coefficient that potentially depends on $S, \eta$ is the granular viscosity, and $\Delta F$ is the unknown upslope-directed-in the negative $x$-direction-and size-ratiodependent force responsible for the lag. The data in Fig. 2.2 provides us with the $S$ dependency of $\lambda_{x}$ and confirms the $1 / \eta$ dependency predicted by Eq. (2.1). Namely, we find a good fit of the data using

$$
\lambda_{x}=a(1 / S-1) / \eta
$$

We calulate the viscosity from a reference flow without intruder via $\eta=|\boldsymbol{\tau}| / \dot{\gamma}$, where $\dot{\gamma}=\partial_{z} u_{x}$ is the shear rate, and $\boldsymbol{\tau}$ is the shear stress. The dimensional fit parameter $a$ 
accounts for the $1 /\left(\pi d_{b}\right)$ in Eq. (2.1), as well as for $\Delta F$, which has dependencies that cannot be straightforwardly extracted from the data in our chute-flow geometry. If certain assumptions are made, which we cannot verify in this geometry, a full expression of $\lambda_{x}$ as a function of the fluid and particle properties can be obtained, as described in Appendix A.1.

Importantly, both the $S$-dependent data and the $z_{p}$-dependent data in Fig. 2.2 can be fitted with the same value for $a$. This fit also demonstrates that

$$
\Delta F(S) / c(S) \propto 1-S .
$$

Further support for the correct scaling of $\lambda_{x}$ is provided in Fig. 2.2(c), where a collapse of the data-except for outliers-is shown when plotting $\eta \lambda_{x}$ as a function of $S$, while Fig. 2.2 $(d)$ shows that all data fall on a line with slope 1.0 when plotting $\eta \lambda_{x}$ as a function of $a(1 / S-1)$.
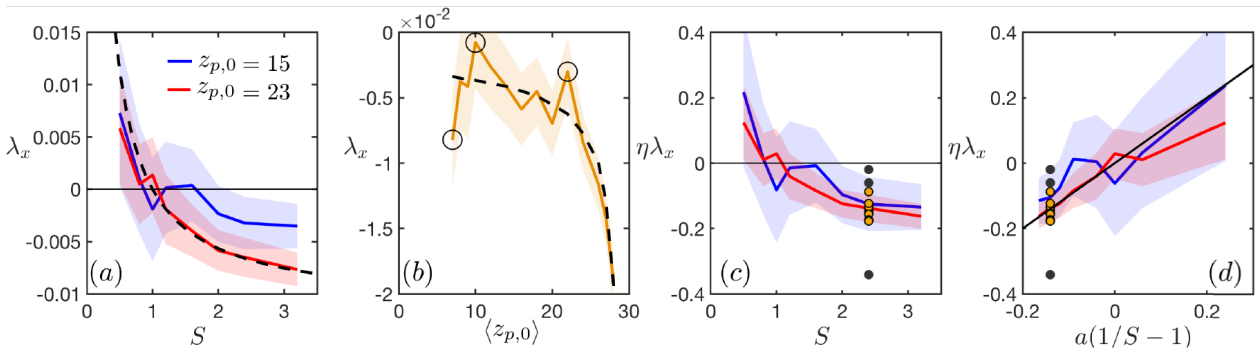

Figure 2.2: (a) The velocity lag $\lambda_{x}$ of the intruder particle as a function of size ratio $S$, for $z_{p, 0}=15$ and $z_{p, 0}=23$. (b) Velocity lag as a function of the vertical positions $\left\langle z_{p, 0}\right\rangle$ of an intruder for $S=2.4$. The dashed lines in $(a)$ and $(b)$ are fits of Eq. (2.2), with $a=0.24$, and $\eta=21.2$. The circles indicate the outliers. (c) The data from $(a)$ and $(b)$ are plotted here as $\eta \lambda_{x}$ versus $S$. The yellow circles are the data from $(b)$, with the black circles indicating the outliers. $(d)$ The data from $(a)$ and $(b)$ are plotted here as $\eta \lambda_{x}$ versus $a(1 / S-1)$. The solid black line has a slope of 1.0. The yellow circles are again the data from $(b)$, with the black circles indicating the outliers.

\subsubsection{Pressure}

We look for the origin of the lag in the pressure field $p$ around the intruder. Figure 2.3(a) shows the cross-section $p\left(\boldsymbol{x}-\boldsymbol{x}_{p}\right)$ at $y-y_{p}=0$ for different size ratios. For $S \leq 1$ the pressure is (almost) hydrostatic, i.e., $p \approx p_{h}=\phi \rho_{p} g_{z}(h-z)$, with a measured $\phi \approx 0.577$. A hydrostatic pressure $p_{h}$, with very little variation in the solids fraction as a function of height, is characteristic for the bulk of this type of flow [45]. For $S>1, p$ deviates from $p_{h}$, and a strong anisotropy manifests itself with a high pressure region at the bottomfront side of the intruder. Pressure variations of lower magnitude also appear around the intruder. This demonstrates that the presence of a large particle modifies the local pressure around it. Although it is known that pulling an object through a granular medium affects the local pressure [46, 47], the situation here is different as the intruder is not pulled but instead is fixed by a spring in the $z$-direction, while it can freely flow in the $x$ - $y$ plane.

In order to isolate the non-hydrostatic effects in the pressure we study $p_{L}=p-$ $p_{h}$. Figure 2.3(b) shows that for $S \leq 1 p_{L}$ is zero, within the fluctuations, while $p_{L}$ in- 
$S=0.5$

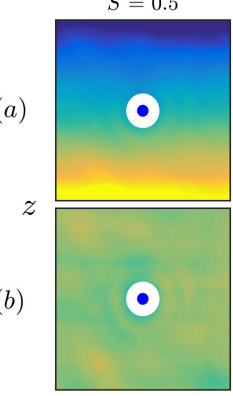

$S=1$

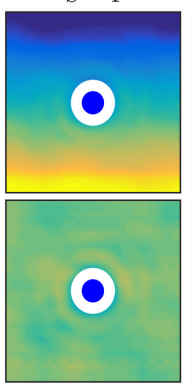

$S=1.6$

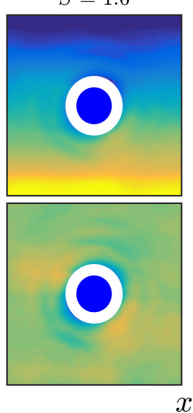

$S=2$
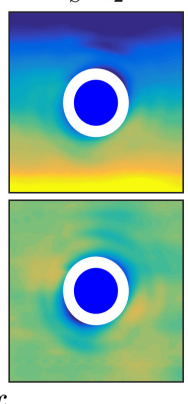

$S=2.4$
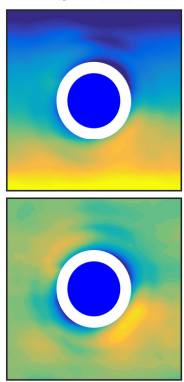

$S=3.2$

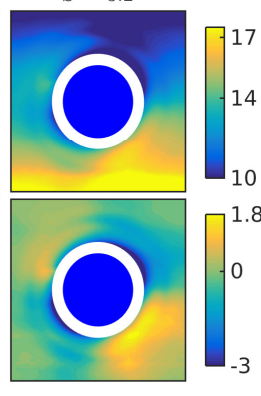

Figure 2.3: ( $a$ ) Cross-sections $p\left(\boldsymbol{x}-\boldsymbol{x}_{p}\right)$ at $y-y_{p}=0$ around the intruder, centred at the origin, for an intruder at $z_{p, 0}=15$. The blue circle (diameter $d_{p}$ ) corresponds to the intruder. The edge of the white circle (diameter $\left.d_{p}+d_{b}\right)$ corresponds to the position of the first layer of bulk particles. (b) Cross-sections $p_{L}(x, 0, z)$, where $p_{L}=p-p_{h}$, around the intruder at $z_{p, 0}=15$.

creases for $S>1$ and is characterised by positive regions (over-pressure) in the lower right and upper left quadrants, and negative regions in the lower left and upper right quadrants. It seems reasonable now to correlate the lift force and the velocity lag to this non-hydrostatic pressure.

\subsubsection{GRANUlar BUOYANCY AND LifT FORCE}

Now that we have found indications that the velocity lag is linked to the local nonhydrostatic pressure field $p_{L}$, we proceed to calculate the lift force $F_{L}$ similar to the way we obtained $p_{L}$, i.e., by subtracting the granular buoyancy force $F_{b}$, that originates from $p_{h}$, from the net contact force on the intruder: $F_{L}=F_{c}-F_{b}$. Various definitions for granular buoyancy forces exist [e.g 37,48], but none account for a dependency on the size ratio. Here we introduce a more general definition that does depend on the size ratio." Taking inspiration from [48] and using our approximation $\phi\left(x_{p}, y_{p}, z_{p}\right)=\phi_{p}$ for the solids fraction at the intruder position, we integrate $p_{h}$ over the surface $A_{V, p}$ of $V_{V, p}$. With the divergence theorem we find:

$$
F_{b}=\int_{A_{V, p}} p_{h} \mathbf{n} \cdot \mathbf{e}_{\mathbf{z}} d A=\phi \rho_{p} g_{z} \int_{V_{V, p}} d V=\phi \rho_{p} g_{z} V_{V, p}
$$

Here $\mathbf{n}$ is the normal outward vector to $A_{V, p}$ and $\mathbf{e}_{\mathbf{z}}$ is the upward unit vector. Substituting $V_{V, p}=V_{p} / \phi_{p}$ we obtain:

$$
F_{b}=\frac{\phi}{\phi_{p}} \rho_{p} g_{z} V_{p}=\frac{\phi}{\phi_{p}} F_{g_{z}}
$$

Effectively this is a generalised size-ratio-dependent buoyancy force Archimedes principle at the particle level defined through an effective density that is equal to the mass of the particle divided by its Voronoi volume. Figure 2.4(a) shows that the measured $\phi_{p}$ strongly depends on $S$ and is bigger than the bulk solids fraction $\phi$ for $S>1$. This means that a larger intruder occupies a larger fraction of its Voronoi volume. The data for $\phi_{p}$ can be fitted by:

$$
\phi_{p}=(\phi-1) S^{c}+1,
$$



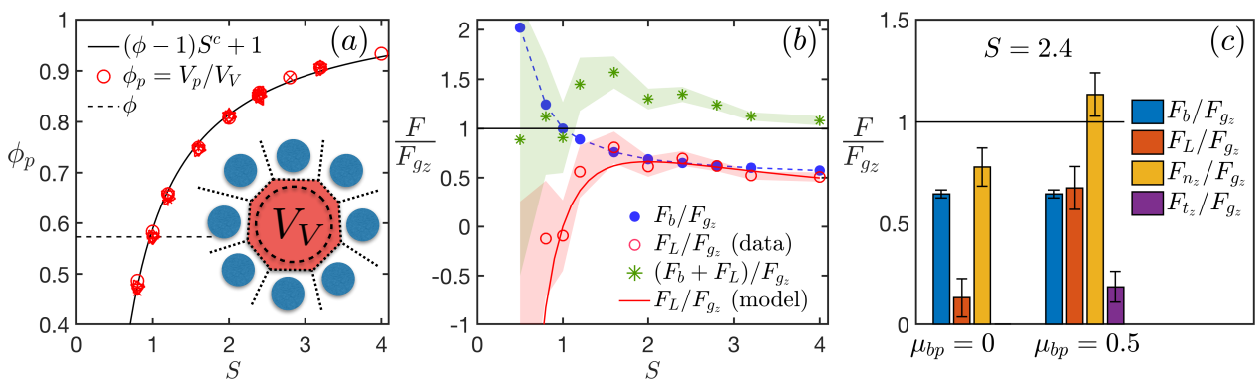

Figure 2.4: (a) Local intruder solids fraction $\phi_{p}$ versus $S$. Different (almost collapsing) symbols correspond to intruders with $\mu_{b p}=0.5, \mu_{b}=0, z_{p, 0}=15, z_{p, 0}=23, \theta=22^{\circ}, 23^{\circ}, 24^{\circ}, 25^{\circ}$, and $26^{\circ}, k=20$ and $k=\infty$. Solid line corresponds to Eq. (2.6) with $c=-1.2$ and $\phi=0.577$. The schematic depicts the Voronoi volume $V_{V}, p$ (dotted octagon) of the intruder (dashed circle). (b) The measured forces $F_{b}, F_{L}$ and $F_{L}+F_{b}$, normalised by $F_{g_{z}}$, for $z_{p, 0}=23$, as well as a fit of $F_{L}$ with Eq. (2.9) (solid red line), with $a=0.24$ and $b=130.0$. The value of $a$ is obtained from the fit in Fig. 2.2( $a$ ). The buoyancy force $F_{b}$ (blue circles) corresponds to Eq. (2.5) with $\phi_{p}$ from (a). (c) The measured forces $F_{b}, F_{L}, F_{n_{z}}$ and $F_{t_{z}}$, normalised by $F_{g_{z}}$, for $S=2.4, \mu_{b p}=0$ and 0.5 , at $z_{p, 0}=15$.

with $c=-1.2$ and $\phi=0.577$.

The ratio $\phi / \phi_{p}$ in $F_{b}$ in Eq. (2.5) has a crucial consequence, namely that for $S>1$ the buoyancy force will be less than the gravity force $F_{g_{z}}=\rho_{p} g_{z} V_{p}$ acting on the particle. This can be seen in Fig. 2.4(b) where $F_{b} / F_{g_{z}}<1$ for $S>1$. When $S=1, \phi$ equals $\phi_{p}$, and the buoyancy force balances $F_{g_{z}}$. In the limit of $S \rightarrow \infty$, we have that $\phi_{p} \rightarrow 1$ and thus $F_{b}$ corresponds to the buoyancy force in a fluid with density $\rho=\phi \rho_{b}$. This generalised buoyancy force differs from the classical Archimedean buoyancy definition $F_{b}=\phi \rho_{p} g_{z} V_{p}$ in a granular fluid, which has two problems: it is independent of $S$, and more critically, predicts that $F_{b}<F_{g_{z}}$ if $S=1$.

Using the new definition for $F_{b}$ we can determine the lift force $F_{L}=F_{c}-F_{b}$, with $F_{c}=F_{n_{z}}+F_{t_{z}}$. Figure 2.4(b) shows that $F_{L} / F_{g_{z}}$ is approximately zero for $S=1$, increases rapidly for $S>1$ and tends to a finite value above $S=2$. The plot of $\left(F_{b}+F_{L}\right) / F_{g_{z}}$ in Fig. 2.4(b) shows that there is an optimal size ratio for segregation, in agreement with experimental findings [11], simulations [49], and theoretical predictions [50].

\subsubsection{SAFFMAN LIFT FORCE}

Here we investigate the relation between the velocity lag of the intruder and the lift force it experiences. Such a relation is known to exist for suspended particles in a fluid: The Saffman lift force on a particle with diameter $d_{p}$ suspended in a fluid of density $\rho$ and viscosity $\eta$ is found to scale with the velocity lag with respect to the surrounding fluid [51, 52]:

$$
F_{\text {Saffman }}=-1.615 \sqrt{\eta|\dot{\gamma}| \rho} \lambda_{x} d_{p}^{2} \operatorname{sgn}(\dot{\gamma}),
$$

where $\dot{\gamma}=\partial_{z} u_{x}\left(z_{p}\right)$ is the shear-rate. Saffman [51] derived this relation taking the fluid properties in the absence of the particle and considered the limit:

$$
\frac{\rho \lambda_{x} d_{p}}{2 \eta} \ll\left(\frac{\rho|\dot{\gamma}| d_{p}^{2}}{4 \eta}\right)^{0.5} \ll 1
$$


where the first term is the Reynolds number for the velocity lag $\mathscr{R}_{\lambda_{x}}$ and the second term is the square root of the shear-rate Reynolds number $\mathscr{R}_{\dot{\gamma}}$. Note that for a granular fluid we can write $\mathscr{R}_{\dot{\gamma}}^{0.5}=I_{\theta} S /(2 \sqrt{\mu})$, if we substitute the granular viscosity $\eta=\mu p|\dot{\gamma}|^{-1}$ and shear rate $|\dot{\gamma}|=I_{\theta} d_{b}^{-1} \sqrt{p / \rho_{p}}$, with $I_{\theta}$ the inertial number [10], and $\mu=\tan \theta$ the bulk friction.

Equation (2.8) physically corresponds to a flow around an intruder that is locally governed by viscous effects $\left(\mathscr{R}_{\lambda_{x}} \ll 1\right)$, but away from the intruder by inertial effects $\left(\mathscr{R}_{\lambda_{x}} \ll \mathscr{R}_{\dot{\gamma}}^{0.5}\right)$. The derivation of the Saffman lift force is not valid when the inertia starts to dominate the local flow around the intruder, and hence the validity is constrained to $\mathscr{R}_{\dot{\gamma}}^{0.5} \ll 1$. Whether Eq. (2.8) is valid for dense granular flows in general remains to be seen, nonetheless it is valid for our current system; we find $\mathscr{R}_{\lambda_{x}}=\mathscr{O}\left(10^{-4}\right)$ using $\rho=\phi \rho_{b}$ and measuring $\eta$ from CG-fields in absence of the intruder, while $\mathscr{R}_{\dot{\gamma}}^{0.5}=I_{22^{\circ}} S /(2 \sqrt{\mu})=$ $\mathscr{O}\left(10^{-1}\right)$ using $I_{22^{\circ}}=0.050$.

\subsubsection{Granular SafFMan Lift Force}

In order to test if a Saffman-like relation exists between $F_{L}$ and $\lambda_{x}$ we fit

$$
F_{L}=-b \sqrt{\eta|\dot{\gamma}| \rho} \lambda_{x} d_{p}^{2} \operatorname{sgn}(\dot{\gamma})
$$

analogous to Eq. (2.7). Here $b$ a dimensionless coefficient that accounts for unknown dependencies, $\lambda_{x}=a(1 / S-1) / \eta$ corresponding to Eq. (2.2), and $\rho=\phi \rho_{b}$. Using $\eta^{-1} \sqrt{\eta|\dot{\gamma}| \rho}$ $=I_{\theta}\left(d_{b} \sqrt{\mu}\right)^{-1}$, Eq. (2.9) can be written as:

$$
F_{L}=-a b I_{\theta} \mu^{-0.5}(1 / S-1) d_{p}^{2} d_{b}^{-1} \operatorname{sgn}(\dot{\gamma}),
$$

demonstrating that the lift force is independent of the flow depth, since $I_{\theta}$ and $\mu$ are constant in a chute flow. We verify that $F_{L}$ is indeed independent of depth (see Appendix A.3), in agreement with the findings of Guillard et al. [37].

We fit Eq. (2.9) to the data of $F_{L}$ in Fig. 2.4(b), using the value for $a$ obtained from the fit in Fig. 2.2, and find that it captures the data well. Subsequently, using the same value for $a$, and the value for $b$ obtained from the fit to $F_{L}$ in Fig. 2.4(b), we fit Eq. (2.9) to the lift force measured as a function of depth in Appendix A.3. This demonstrates that Eq. (2.9) is the correct scaling between the lift force, size ratio, viscosity and velocity lag at constant inclination angle in a chute flow. The fact that this scaling is Saffman-like suggests that inertial effects could lie at the origin of the segregation of large particles in dense granular flows with pressure and velocity gradients in the limit of low largeparticle concentrations.

To provide further support for our finding that the generalised buoyancy force does not support the weight of a large intruder $(S>1)$ we set the intruder-bulk friction $\mu_{b p}$ to zero and find that $F_{L}$ is reduced, as shown in Fig. 2.4(c). Critically, this leads to a large none-frictional intruder sinking instead of rising, as found recently also experimentally: lower-friction particles sink below higher-friction particles in mono-disperse granular flows [53]. Since the net contact force $F_{c}=F_{n_{z}}+F_{t_{z}}$ on the intruder is lower than $F_{g_{z}}$, the buoyancy $F_{b}$ must also be less than $F_{g_{z}}$. Note that in Fig. 2.4(c) the spring force brings the force balance back to zero: $F_{s p}-F_{g_{z}}+F_{c}=F_{s p}-F_{g_{z}}+F_{b}+F_{L}=0$. Interestingly, the lift force does not completely disappear, indicating it should have both a geometric 
and frictional component. We verified that $p_{L}$ is reduced but does not disappear for frictionless particles.

\subsection{Conclusions}

We report that a single large particle in a dense granular flow is surrounded by an anisotropic, non-hydrostatic pressure field. This coincides with our observations of a velocity lag and a lift force, coupled through a Saffman-like relation, Eq. (2.9), causing the particle to rise against gravity. These findings suggest that the mechanism of squeeze expulsion [15] - which is often invoked to qualitatively explain the segregation of large particles in dense granular flows-is the granular equivalent of the Saffman effect; an inertial lift force in an otherwise strongly viscous bulk flow [51, 52].

A possible physical interpretation of the Saffman effect for a granular fluid could be that in our mostly viscous and slow flow, but with a finite, considerable inertial number, a large intruder disturbs the local (Bagnold) flow profile. Because the bulk inertial effects, which are proportional to the strain-rate, are not negligible, the rheology driven by the velocity gradient - associated with the inertially generated, but perturbed velocity field-produces an anisotropy of the pressure field, which creates both the lift force and the drag force responsible for the velocity lag.

The decomposition of the contact force on the intruder into a lift force and generalised buoyancy force is essential to the preceding analysis. Moreover, it provides a physical explanation for the sinking of very large intruders $[54,55]$, as well as for the optimal size ratio for segregation $[11,50]$ and the unexplained trend of the total contact force $F_{c}(S)$ in Fig. 6 of [37]. Namely, if we consider the limit of Eq. (2.9) at large size ratios, we see that the lag approaches a constant value, while the buoyancy force approaches a fluid buoyancy with density $\rho=\phi \rho_{b}$. Gravity will then outgrow the total upward force and the particle will sink.

Further studies could address the following questions: If inertial effects indeed lie at the origin of size segregation of large intruders at low large-particle concentration, they could potentially also play a role in slow, dense, polydisperse granular flows with more than one intruder. Thus, the variation of the lift force when the large-particle concentration increases could be investigated. Furthermore, in order to validate the Saffman relation for granular flows changing the stress gradient in the flow would be necessary. This can be done by using other geometries, for example, such as the one used by Guillard et al. [37]. Last but not least, the reported sinking of a large intruder with zero intruderbulk friction $\mu_{b p}$ hints at the importance of particle properties.

Drag forces on a free-flowing object in granular media, in contrast to a dragged object, have received little attention [48]. Our findings suggest that the Stokesian drag, found by Tripathi and Khakhar [48] for a heavy sinking mono-disperse intruder, plays an important role in the rising of large intruders (see Appendix A.1). A continued effort to determine all drag forces acting on free-flowing particles is important for the rheology of granular flows in general, but foremost because drag is a cornerstone of models for particle-size segregation in dense granular flows.

In order to unify Eq. (2.9) with the scaling laws found by Guillard et al. [37] and develop a multi-scale model for the segregation of large intruders in dense granular flows the lag will have to be expressed in terms of $\lambda_{x}=f(\partial p / \partial z, \partial|\boldsymbol{\tau}| / \partial z, \dot{\gamma}, \partial \dot{\gamma} / \partial z)$, where $\boldsymbol{\tau}$ is 
the shear stress. This is far from trivial: The dependency of all variables on $z$ and $\theta$ is very weak and the range of accessible pressure gradients, inertial numbers, etc., is very limited in steady state chute flows (inclination angles that are too large lead to accelerating flows, whereas too small angles lead to stopping of the flow $[18,45])$. To demonstrate the dependencies more convincingly, one should disentangle pressure and tangential stress and show that the Saffman-like relation still holds. In order to do so, a completely different flow geometry needs to be considered, which, however, goes beyond the scope of the present study. Finally, for a formal proof that a Saffman-like relation holds in granular fluids, the analytical derivation by Saffman could be repeated for a granular rheology.

\section{REFERENCES}

[1] K. van der Vaart, M. P. van Schrojenstein Lantman, T. Weinhart, S. Luding, C. Ancey, and A. R. Thornton, Segregation of large particles in dense granular flows suggests a granular saffman effect, Physical Review Fluids 3, 074303 (2018).

[2] I. S. Aranson and L. S. Tsimring, Patterns and collective behavior in granular media: Theoretical concepts, Reviews of Modern Physics 78, 641 (2006).

[3] J. C. Williams, The segregation of particulate materials. a review, Powder technology 15, 245 (1976).

[4] A. Rosato, K. J. Strandburg, F. Prinz, and R. H. Swendsen, Why the brazil nuts are on top: Size segregation of particulate matter by shaking, Physical Review Letters 58, 1038 (1987).

[5] J. M. Ottino and D. V. Khakhar, Mixing and segregation of granular materials, Annual Review of Fluid Mechanics 32, 55 (2000).

[6] J. Duran, J. Rajchenbach, and E. Clément, Arching effect model for particle size segregation, Physical Review Letters 70, 2431 (1993).

[7] S. Dippel and S. Luding, Simulation on size segregation: Geometrical effects in the absence of convection, Journal de Physique I 5, 1527 (1995).

[8] J. B. Knight, H. M. Jaeger, and S. R. Nagel, Vibration-induced size separation in granular media: The convection connection, Physical Review Letters 70, 3728 (1993).

[9] GDR-MiDi, On dense granular flows, The European Physical Journal E 14, 341 (2004).

[10] P. Jop, Y. Forterre, and O. Pouliquen, A constitutive law for dense granular flows, Nature 441, 727 (2006).

[11] L. A. Golick and K. E. Daniels, Mixing and segregation rates in sheared granular materials, Physical Review E 80, 042301 (2009).

[12] Y. Fan and K. M. Hill, Shear-driven segregation of dense granular mixtures in a splitbottom cell, Physical Review E 81, 041303 (2010). 
[13] Y. Fan and K. M. Hill, Theory for shear-induced segregation of dense granular mixtures, New Journal of Physics 13, 095009 (2011).

[14] J. A. Drahun and J. Bridgwater, The mechanisms of free surface segregation, Powder Technology 36, 39 (1983).

[15] S. B. Savage and C. K. K. Lun, Particle size segregation in inclined chute flow of dry cohesionless granular solids, Journal of Fluid Mechanics 189, 311 (1988).

[16] C. R. K. Windows-Yule, B. J. Scheper, A. J. van der Horn, N. Hainsworth, J. Saunders, D. J. Parker, and A. R. Thornton, Understanding and exploiting competing segregation mechanisms in horizontally rotated granular media, New Journal of Physics $\mathbf{1 8}$, 023013 (2016).

[17] K. M. Hill and D. S. Tan, Segregation in dense sheared flows: gravity, temperature gradients, and stress partitioning, Journal of Fluid Mechanics 756, 54 (2014).

[18] T. Weinhart, S. Luding, A. R. Thornton, A. Yu, K. Dong, R. Yang, and S. Luding, From discrete particles to continuum fields in mixtures, in AIP conference proceedings, Vol. 1542 (AIP, 2013) pp. 1202-1205.

[19] L. Staron and J. C. Phillips, Stress partition and microstructure in size-segregating granular flows, Physical Review E 92, 022210 (2015).

[20] D. R. Tunuguntla, T. Weinhart, and A. R. Thornton, Comparing and contrasting sizebased particle segregation models, Computational Particle Mechanics 4, 387 (2017).

[21] S. Wiederseiner, N. Andreini, G. Épely-Chauvin, G. Moser, M. Monnereau, J. M. N. T. Gray, and C. Ancey, Experimental investigation into segregating granular flows down chutes, Physics of Fluids 23, 013301 (2011).

[22] L. Staron and J. C. Phillips, Segregation time-scale in bi-disperse granular flows, Physics of Fluids 26, 033302 (2014).

[23] M. Harrington, J. H. Weijs, and W. Losert, Suppression and emergence of granular segregation under cyclic shear, Physical Review Letters 111, 078001 (2013).

[24] L. Staron and J. C. Phillips, How large grains increase bulk friction in bi-disperse granular chute flows, Computational Particle Mechanics 3, 367 (2016).

[25] A. N. Edwards and N. M. Vriend, Size segregation in a granular bore, Physical Review Fluids 1, 064201 (2016).

[26] L. Jing, C. Y. Kwok, and Y. F. Leung, Micromechanical origin of particle size segregation, Physical Review Letters 118, 118001 (2017).

[27] J. M. N. T. Gray and A. R. Thornton, A theory for particle size segregation in shallow granular free-surface flows, Proceedings of the Royal Society of London A: Mathematical, Physical and Engineering Sciences 461, 1447 (2005). 
[28] A. R. Thornton, J. M. N. T. Gray, and A. J. Hogg, A three-phase mixture theory for particle size segregation in shallow granular free-surface flows, Journal of Fluid Mechanics 550, 1 (2006).

[29] B. Marks, P. Rognon, and I. Einav, Grainsize dynamics of polydisperse granular segregation down inclined planes, Journal of Fluid Mechanics 690, 499 (2012).

[30] D. R. Tunuguntla, O. Bokhove, and A. R. Thornton, A mixture theory for size and density segregation in shallow granular free-surface flows, Journal of Fluid Mechanics 749, 99 (2014).

[31] K. van der Vaart, P. Gajjar, G. Epely-Chauvin, N. Andreini, J. M. N. T. Gray, and C. Ancey, Underlying asymmetry within particle size segregation, Physical Review Letters 114, 238001 (2015).

[32] P. Gajjar and J. M. N. T. Gray, Asymmetric flux models for particle-size segregation in granular avalanches, Journal of Fluid Mechanics 757, 297 (2014).

[33] K. Kamrin and G. Koval, Nonlocal constitutive relation for steady granular flow, Physical Review Letters 108, 178301 (2012).

[34] D. L. Henann and K. Kamrin, A predictive, size-dependent continuum model for dense granular flows, Proceedings of the National Academy of Sciences 110, 6730 (2013).

[35] R. J. Phillips, R. C. Armstrong, R. A. Brown, A. L. Graham, and J. R. Abbott, A constitutive equation for concentrated suspensions that accounts for shear-induced particle migration, Physics of Fluids 4, 30 (1992).

[36] F. Boyer, O. Pouliquen, and É. Guazzelli, Dense suspensions in rotating-rod flows: normal stresses and particle migration, Journal of Fluid Mechanics 686, 5 (2011).

[37] F. Guillard, Y. Forterre, and O. Pouliquen, Scaling laws for segregation forces in dense sheared granular flows, Journal of Fluid Mechanics 807 (2016).

[38] A. R. Thornton, D. Krijgsman, A. Voortwis, V. Ogarko, S. Luding, R. Fransen, S. Gonzalez, O. Bokhove, O. Imole, and T. Weinhart, A review of recent work on the discrete particle method at the university of twente: An introduction to the open-source package mercurydpm, in DEM 6 - International conference on DEMs (Colorado School of Mines, 2013).

[39] T. Weinhart, D. R. Tunuguntla, M. P. van Schrojenstein-Lantman, A. J. van der Horn, I. F. C. Denissen, C. R. Windows-Yule, A. C. de Jong, and A. R. Thornton, Mercurydpm: A fast and flexible particle solver part a: Technical advances, in Proceedings of the 7th International Conference on Discrete Element Methods (Springer, 2017) pp. 1353-1360.

[40] P. A. Cundall and O. D. L. Strack, A discrete numerical model for granular assemblies, Geotechnique 29, 47 (1979). 
[41] T. Weinhart, R. Hartkamp, A. R. Thornton, and S. Luding, Coarse-grained local and objective continuum description of three-dimensional granular flows down an inclined surface, Physics of Fluids 25, 070605 (2013).

[42] D. R. Tunuguntla, A. R. Thornton, and T. Weinhart, From discrete elements to continuum fields: Extension to bidisperse systems, Computational Particle Mechanics 3, 349 (2016).

[43] I. Goldhirsch, Stress, stress asymmetry and couple stress: from discrete particles to continuous fields, Granular Matter 12, 239 (2010).

[44] C. Rycroft, Voro++: A three-dimensional voronoi cell library in c++, Lawrence Berkeley National Laboratory (2009).

[45] T. Weinhart, A. R. Thornton, S. Luding, and O. Bokhove, Closure relations for shallow granular flows from particle simulations, Granular Matter 14, 531 (2012).

[46] F. Guillard, Y. Forterre, and O. Pouliquen, Lift forces in granular media, Physics of Fluids 26, 043301 (2014).

[47] F. Guillard, Y. Forterre, and O. Pouliquen, Origin of a depth-independent drag force induced by stirring in granular media, Physical Review E 91, 022201 (2015).

[48] A. Tripathi and D. V. Khakhar, Numerical simulation of the sedimentation of a sphere in a sheared granular fluid: a granular stokes experiment, Physical Review Letters 107, 108001 (2011).

[49] A. Thornton, T. Weinhart, S. Luding, and O. Bokhove, Modeling of particle size segregation: calibration using the discrete particle method, International Journal of Modern Physics C 23, 1240014 (2012).

[50] J. M. N. T. Gray and C. Ancey, Multi-component particle-size segregation in shallow granular avalanches, Journal of Fluid Mechanics 678, 535 (2011).

[51] P. G. T. Saffman, The lift on a small sphere in a slow shear flow, Journal of Fluid Mechanics 22, 385 (1965).

[52] H. A. Stone, Philip Saffman and viscous flow theory, Journal of Fluid Mechanics 409, 165 (2000).

[53] K. A. Gillemot, E. Somfai, and T. Börzsönyi, Shear-driven segregation of dry granular materials with different friction coefficients, Soft Matter 13, 415 (2017).

[54] N. Thomas, Reverse and intermediate segregation of large beads in dry granular media, Physical Review E 62, 961 (2000).

[55] G. Félix and N. Thomas, Evidence of two effects in the size segregation process in dry granular media, Physical Review E 70, 051307 (2004). 



\section{3}

\section{THE BUOYANCY ForCE ON A SPHERICAL INTRUDER IN DENSE GranUlar Flows}

Archimedes' principle states that an object in a fluid feels an upward buoyant force proportional to the weight of the displaced fluid. In dense granular flows a correction to this principle is required due to a lack of scale-separation between an intruding object and bulk particle size. Here we derive a correction based on micro structure and forces and incorporate force chains and force fluctuations for both sheared and non-sheared granular systems. We observe that as a large intruder approaches the size of a bulk particle, the number of contacts per surface area increases. More contacts around the spherical intruder yields an increase in buoyancy force. This mechanism can be accurately captured by applying Archimedes' principle to the Voronoi volume of the object. The approach shows that the correction does not only apply to the buoyancy force, but it applies to all mechanisms like lift and drag forces. This is important when developing a more complete model for mixing and segregation.

\subsection{INTRODUCTION}

An intruder in a granular flow is an object that differs from bulk particles in size, density or other properties. Predicting the trajectory of such an intruder in granular flows is important in industrial processes such as mixing. A lift and drag force based model could capture the trajectory of the intruder, similar to the Basset-Boussinesq-Oseen-equation for a particle in a classical viscous fluid [1].

Drag forces have been measured when increasing the intruder density, while keeping the size similar to the bulk particles. Simulations in chute flows have shown the presence of a granular equivalent to the Stokes drag force [2]. This drag force depends linearly on the density ratio between bulk particles and intruder. When higher inertial flows are considered, the drag force shows non-linear scaling with the density ratio [3]. 
Lift forces on intruders have been observed when increasing the size of the intruder. A recently developed force model considers the total vertical force, yielding scaling laws that purely depend on the derivative of the pressure and shear stress [4] . In Chapter 2 this vertical force was decomposed in a buoyancy force and a lift force by splitting the pressure $p$ around the intruder in a hydrostatic and a lift part, $p=p_{h}+p_{L}$.

An aspect that has not yet received much attention is Archimedes' principle. This principle states that an object in a fluid feels an upward force proportional to the weight of the displaced fluid and is called the buoyancy force. If the size of the intruder is close to the size of the bulk particles, it is non-trivial to identify how much volume is displaced by the intruder due to a lack of scale separation. Only in limiting cases can the buoyancy force be estimated and in this work two cases are considered.

The first limiting case case is $S \rightarrow \infty$, where the intruder is so large that the granular flow can be assumed to be a continuum and thus the classical buoyancy force is expected. Experiments for larger intruders have confirmed the classical buoyancy force in locally stirred systems [5] and in a (horizontally vibrated) fluidised bed [6]. In the latter work the smallest size ratio's used were $S=r_{p} / r_{b}=8$, with radii $r_{p}$ for intruder and $r_{b}$ for bulk particle, thus this limit is obtained before $\infty$.

The second limiting case is $S=1$ where the intruder is exactly equal to the bulk particles. In these cases an increase of the buoyancy force is observed [2], deviating from Archimedes principle. Since all bulk particles are similar, the effective displaced volume can be determined by distributing all void space equally among all bulk particles. Another system where a deviation from Archimedes' law is observed is in binary suspensions, where dense particles can be on top of lighter particles [7]. This was observed for large size ratio's, in contrast to the current system, implying a different mechanism.

The buoyancy force on an intruder for $S \geq 1$ was generalised for all $S$ by applying a Voronoi volume correction to the buoyancy force [8]. Instead of using the volume of the intruder in Archimedes' principle, the Voronoi volume is used. This geometrical correction naturally satisfies the two limiting cases of $S \rightarrow 1$ and $S \rightarrow \infty$. However, this correction raises a few questions. Firstly, it is still unclear what the physical mechanism behind this correction is. Secondly, if the buoyancy force requires a correction based on the Voronoi volume, do drag and lift forces also require such a correction? Finally, on a more philosophical level: at what size ratio does the intruder behave like an object in a granular fluid, i.e. when can scale separation be assumed between the intruder and bulk particles.

To answer these questions, we adopt a similar decomposition as in Chapter 2. However, instead of focussing on the continuum pressure, we focus on the contact forces by disentangling the contact structure (fabric) and contact forces similar to Ref. [9]. This separation of structure and contact forces facilitates a decomposition of the contact forces without decomposing the structure. This approach has been used to study bulk behaviour of granular materials in 2D [10,11] and 3D [12] for constitutive modelling.

In this work, the contact structure and contact force fields in dense granular flows for intruders with $S \geq 1$ is analysed in the well-known chute flow system [13, 14]. Details of the simulation and analysis methods are given in section 3.2 and in section 3.3 the flow profile of the monodisperse flow without intruder is discussed. The buoyancy theory of a spherical intruder in a granular flow is elaborated in section 3.4. Assumptions 
made in the theory are validated in section 3.5 and finally in section 3.6 the results and implications of the theory will be discussed and an outlook is given.

\subsection{MeTHODS}

First the simulation method is discussed in section 3.2.1. In section 3.2.2 the analysis method is elaborated on how to obtain the spatial structure on the surface of an intruder. Converting simulation data of discrete particles in continuum fields is explained in section 3.2.2.

\subsubsection{Simulation SETUP}

The simulation setup is similar to chapter 2. A gravity driven granular flow down an incline (chute flow) with angle $\theta_{c}=22^{\circ}$ is simulated. Periodic boundaries are applied in the $x$ - and $y$-directions. This setup is chosen as it produces a velocity field which is shearing in only one plane, the $x z$-plane. This is a well studied system, see Refs. [13-15] and references therein. The granular chute flow is simulated using the discrete particle method (DPM). The system is non-dimensionalised such that the non-dimensional bulk particle diameter is $d_{b}=1$, density $\rho_{b}=6 / \pi$ and the gravitational acceleration $g=1$. The simulation box has a size $(L x, L y, L z)=(20,8.9,40) d_{b}$, The gravity is given by $\boldsymbol{g}=$ $\left[\sin \left(\theta_{c}\right), 0,-\cos \left(\theta_{c}\right)\right]$. A rough bottom is composed of slightly larger than bulk particles, with a diameter of $1.7 d_{b}$. Details for the creation method of the rough bottom can be found in Ref. [14].

Roughly 6000 dry frictional particles are simulated yielding an average flow height of $h \approx 30 d_{b}$. Contacts between particles are modeled using a linear spring-dashpot model $[15,16]$ with linear elastic and linear dissipative contributions for the normal forces between particles. Particle properties and contact parameters are given in Table 3.1. The stiffness and dissipation of the contact laws are computed through the analysis of two colliding particles [17]. The tangential, sliding and rolling friction between bulk particles are all equal to the friction coefficient $\mu_{b b}$ in Table 3.1. The friction between intruder and bulk particles, $\mu_{p b}$ is taken similar to $\mu_{b b}$. Simulations are performed by the open-source software package MercuryDPM (MercuryDPM.org; [18, 19]).

An intruder with size ratio $S$ is introduced in the flow at a height of $z_{p, 0}=23$ and to keep the intruder at approximately the same height, a restoring spring is introduced [4] in the $z$-direction, while it can freely flow in the $x$ - and $y$-directions. The spring stiffness of the restoring spring is set to $k_{s}=20$.

\subsubsection{STRUCTURAL ANALYSIS}

Measuring the structure and contact force fields on the surface of the intruder is done by tessellating the surface of the intruder with mesh elements. Here we use a spherical coordinate system with $(r, \theta, \phi)$, respectively the radial-, azimuthal- and polar-direction where $\theta \in[0,2 \pi)$ and $\phi \in[0, \pi]$. The angles are uniformly discretised with $\Delta \theta=\frac{2 \pi}{n_{\theta}}$ and $\Delta \phi=\frac{\pi}{n_{\phi}}$, where $n_{\theta}$ and $n_{\phi}$ are the number of elements in the $\theta$ - and $\phi$-direction, respectively. The surface area of a mesh element is denoted by $\Delta A_{i j}$, where the indices $i$ and $j$ indicate the mesh coordinates $\theta_{i}$ and $\phi_{j}$. To obtain good statistical data, $N_{t}=3400$ time snaps were used with 9724 contacts for the $S=1$ case. A grid of $n_{\theta}=20$ and $n_{\phi}=10$ 


\begin{tabular}{|l|l|l|}
\hline Parameter & Symbol & value \\
\hline bulk particle diameter & $d_{b}$ & 1.0 \\
Intruder particle diameter & $S d_{b}$ & $1.0-3.2$ \\
particle density & $\rho_{b}$ & $\pi / 6$ \\
\hline Restitution coefficient & $e_{r}$ & 0.1 \\
Contact duration & $t_{c}$ & 0.005 \\
Friction & $\mu_{b b}, \mu_{p b}$ & 0.5 \\
\hline
\end{tabular}

Table 3.1: Dimensionless particle properties and contact model parameters. Contact duration and restitution coefficient set the stiffness and dissipation of the particles, see Ref [17].

resulted in a detailed yet reasonably smooth surface. Larger sized intruders encounter more contacts due to increasing coordination number improving the statistical results.

The average number of contacts per in area $E$ in $\Delta A_{i j}$ is given by

$$
\hat{E}_{i j}=\frac{1}{N_{t} \Delta A_{i j}} \sum_{c} 1, \quad c \in \Delta A_{i j}
$$

where the contact $c$ needs to be within element $\Delta A_{i j}$ and the hat indicates it is a measured quantity. The hat indicates a measured value as opposed to the theoretical field later discussed in section 3.4. Summation over the surface area yields the coordination number $\mathcal{Z}$,

$$
\sum_{i} \sum_{j} \hat{E}_{i j} \Delta A_{i j}=\mathfrak{Z}
$$

The average local force on an element $\hat{\boldsymbol{F}}$ can obtained by summation of all local contact forces $\hat{\boldsymbol{f}}_{c}$

$$
\hat{\boldsymbol{F}}_{i j}=\frac{1}{N_{t}} \sum_{c} \hat{\boldsymbol{f}}_{c}, \quad c \in \Delta A_{i j} .
$$

Note that summing over all elements yields the total average force on the intruder,

$$
\hat{\boldsymbol{F}}_{t o t}=\sum_{i} \sum_{j} \hat{\boldsymbol{F}}_{i j}
$$

To obtain the average contact force $\hat{\boldsymbol{F}}_{c}$ in a single element $\Delta A_{i j}$, the average local force on an element is divided by the number of contacts, $\hat{E}_{i j} \Delta A_{i j}$,

$$
\hat{\boldsymbol{F}}_{c_{i j}}=\frac{\hat{\boldsymbol{F}}_{i j}}{\hat{E}_{i j} \Delta A_{i j}} .
$$

The total force on the intruder particle can be expressed as

$$
\hat{\boldsymbol{F}}_{t o t}=\sum_{i} \sum_{j} \hat{\boldsymbol{F}}_{c_{i j}} \hat{E}_{i j} \Delta A_{i j}
$$

In a situation where the grid can be taken infinitely small this equation would reduce to the integral of Eq. (3.20) in section 3.4.3. 


\subsubsection{COARSE GRAINING}

Turning discrete particle data, such as particle position and velocity, into continuum fields like stress $\boldsymbol{\sigma}$, volume fraction $\phi_{b}$ and velocity vector $\left(u_{x}, u_{y}, u_{z}\right)$ is done by applying the coarse graining analysis technique [20]. This technique takes "microscopic" point values of particles and smoothes them by applying a kernel function $\mathbb{W}$. In this work we use a Heaviside kernel with a smoothing length scale of $d_{b} / 2$. For details of the analysis the reader is referred to [21] and references therein. The coarse graining analysis is performed with MercuryCG [19].

The sign convention of stress in the granular material community and the fluids community is different. In this work the fluid convention is adopted where compressional stress is negative. The stress is therefore defined as $\boldsymbol{\sigma}=\boldsymbol{\tau}-p \boldsymbol{I}$, where $\boldsymbol{\tau}$ is the shear stress tensor and $p$ the pressure.

\subsection{Simulations}

A reference bulk simulation is performed with $S=1$ to investigate the behaviour of the homogeneous granular fluid. In Fig 3.1 the volume fraction, velocity and stress profiles are shown. A practically constant volume fraction in the bulk of the flow is observed in panel a), with $\phi_{b} \approx 0.577$. The pressure, due to the constant density, appears to be linear in $z$ and so is $\tau_{x z}$; these trends are in agreement with Ref. [14]. The analytical solution of a Bagnold profile is used as model [22], where the the density is assumed constant yielding hydrostatic pressure,

$$
p^{R}=\phi_{b} \rho_{b} g h\left(1-\frac{z}{h}\right) \cos \left(\theta_{c}\right),
$$

where $\rho=\phi \rho_{p}$ and $R$ implies it is a reference flow without intruder. Note that the measured pressure is $\left(1-\alpha_{z z}\right) \sigma_{z z}$ with $\alpha_{z z}=0.05$ due to anisotropy in the stress, in agreement with Ref. [14]. The measured stress $\sigma_{z z}$ is lithostatic which explains the slight deviation between the measurements and the model, see panel $\mathrm{c}$ ). The shear stress $\tau_{x z}$ is

$$
\tau_{x z}^{R}=\phi_{b} \rho_{b} g h\left(1-\frac{z}{h}\right) \sin \left(\theta_{c}\right)
$$

and the Bagnold velocity profile is given by

$$
u_{x}^{R}=\frac{2}{3} I_{\theta_{c}} \sqrt{g d_{b} \cos \left(\theta_{c}\right) \frac{h^{3}}{d_{b}^{3}}}\left[1-\left(1-\frac{z}{h}\right)^{3 / 2}\right] .
$$

Here the (constant) inertial number $I_{22^{\circ}}=0.049$ is used as a fit parameter and $h \approx 30$ is the height of the flow.

The model is in good agreement with the measurements, as can be seen from Fig 3.1. The field profiles at the surface and at the base of the chute flow considerably deviate from the Bagnold model, but in the bulk very good agreement is obtained.

\subsection{THEORY}

This section discusses and generalises the Archimedes principle for an object in a granular flow such that it is valid for all objects equal to or larger than the size of the bulk 

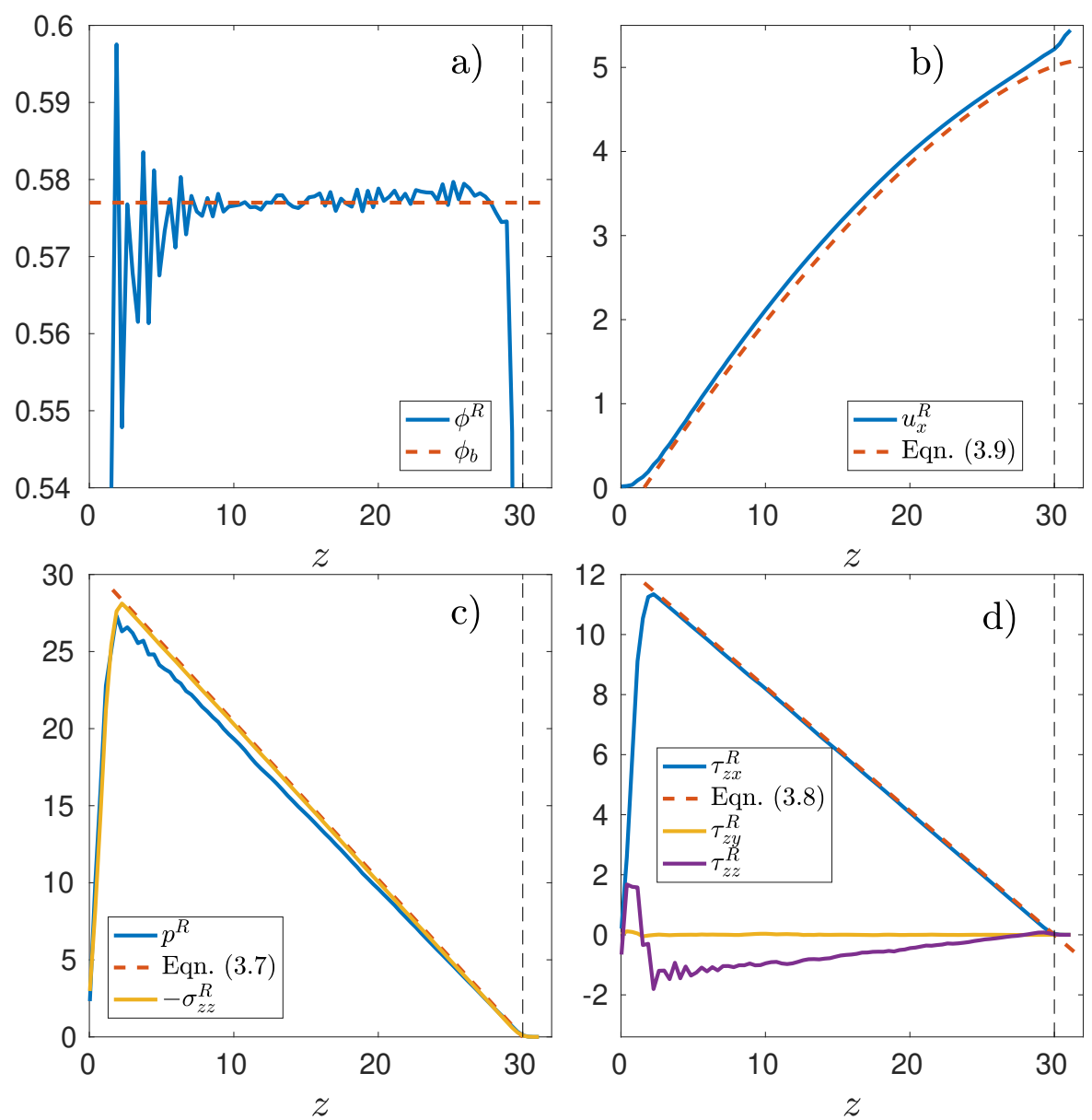

Figure 3.1: Coarse grained continuum fields of a chute flow simulation with angle $\theta_{c}=22^{\circ}$. a) Volume fraction, b) Velocity in the $x$-direction, c) pressure and d) shear stress. The dashed black lines indicate the height of the flow, i.e. the free surface. Coarse grain kernel is Heaviside with a smoothing length scale of $w=d_{b} / 2$. 
particles. To this end the buoyancy force is defined and discussed in section 3.4.1. Two approaches are used to support the buoyancy force definition, a Voronoi tessellation approach in section 3.4.2 and an approach based on the surface contact density in section 3.4.3.

\subsubsection{BUOYANCY FORCE}

Given the stress tensor in a fluid $\boldsymbol{\sigma}=\boldsymbol{\tau}-p \boldsymbol{I}$, the force on a general object inside the fluid can be calculated by integrating the stress over its surface,

$$
\boldsymbol{F}=\int_{A_{p}} \boldsymbol{\sigma} \boldsymbol{n} d A
$$

where $\boldsymbol{n}$ is the normal outward unit vector. The buoyancy force component $\boldsymbol{F}_{b}$ of the total force is generally defined in a fluid as the force caused by the presence of a pressure gradient in the undisturbed reference flow. To distinguish between this reference pressure and the actual pressure on the intruder, the superscript $R$ indicates the reference flow. The buoyancy force is then defined as

$$
\boldsymbol{F}_{b}=: \int_{A_{p}}-p^{R} \boldsymbol{n} d A=-\int_{V_{p}} \nabla p^{R} d V,
$$

where the divergence theorem has been used to obtain a volume integral.

\section{A. GRANULAR BUOYANCY FORCE FOR $S \rightarrow \infty$}

In a chute flow setup, consider the force in the $z$-direction by applying the divergence theorem on the reference stress $\sigma^{R}$ and noting that derivatives in the $x$ - and $y$-direction are zero, yielding

$$
F_{z}^{R}=\boldsymbol{e}_{z} \cdot \int_{A_{p}} \boldsymbol{\sigma}^{R} \boldsymbol{n} d A=\int_{V_{p}} \frac{\partial\left(\tau_{z z}^{R}-p^{R}\right)}{\partial z} d V .
$$

In a normal fluid with an isotropic stress tensor the term $\tau_{z z}^{R}$ is zero, however in a granular flow this term depends on the pressure, $\tau_{z z}^{R}=-\alpha_{z z} p^{R}$, see [15]. While in a fluid the definition of a buoyancy force normally is given by Eq. (3.11), we adopt a buoyancy force definition including this anisotropic term by defining the hydrostatic pressure as

$$
p_{h}=-\sigma_{z z}^{R}=\phi_{b} \rho_{b} g \cos (\theta)[h-z],
$$

such that the buoyancy force is given by

$$
F_{b}^{S \rightarrow \infty}=-\int_{V_{p}} \frac{\partial p_{h}}{\partial z} d V=\phi_{b} \rho_{b} g \cos (\theta) V_{p} .
$$

Note that this definition of buoyancy is equal to the classical buoyancy, which assumes fluid particles are very small compared to the intruder. Interestingly, this buoyancy definition has already been observed for $S>8$ [6]. 


\section{B. GRANULAR BUOYANCY FORCE FOR $S \rightarrow 1$}

If Eq. (3.14) is applied to an object the size of a granular fluid particle, $S=1$, the total force balance in the $z$-direction can be written as

$$
F_{b}^{S \rightarrow \infty}+F_{g}=\left(\phi_{b}-1\right) \rho_{b} g \cos (\theta) V_{p} \neq 0 .
$$

As the buoyancy force and gravity force are not in equilibrium it would imply this particle is not in a steady state, while the flow is. Hence, the expected buoyancy force, Eq. (3.14), is unable to compensate the gravity force other than for $S \rightarrow \infty$. The magnitude difference between $F_{g}$ and $F_{b}^{S \rightarrow \infty}$ can not be explained by the (weak) anisotropy in the stress.

The buoyancy force at $S=1$ can be reasoned by an equality argument. The hydrostatic pressure in the flow is defined at all points, including any void spaces. The pressure in these void spaces can be attributed to all particles equally, since all particles have the same size. Including the pressure inside the voids in Eq. (3.12) implies integration over an effective volume. The effective volume of the particle then becomes $V / N_{p}$, where $V$ is the volume of the whole flow and $N_{p}$ is the number of particles. With the definition of solids fraction in a homogeneous flow (See Fig. 3.1(a)), $\phi_{b}=\left(N_{p} V_{p} / V\right)$, the effective volume can be written as $V_{p} / \phi_{b}$. The buoyancy force at $S=1$ therefore becomes

$$
F_{b}^{S=1}=\frac{1}{\phi_{b}} F_{b}^{S \rightarrow \infty}=-F_{g},
$$

effectively balancing the gravity force in Eq. 3.15. Although this definition is generally accepted [2, 23], it remains a remarkable observation that a buoyancy force definition does not obey Archimedes' law.

\section{GENERALISED GRANULAR BUOYANCY FORCE}

In order to fully understand the buoyancy force on an intruder, it is important to find a common definition that couples both Eq. (3.14) and Eq. (3.16) as a function of $S$. Therefore, a non-dimensional $S$-dependent buoyancy function $B(S)$ is introduced,

$$
B(S)=\frac{F_{b}(S)}{\phi_{b} F_{g_{z}}},
$$

where the function $B(S)$ has to satisfy $B(S=1)=\frac{1}{\phi_{b}}$ and $B(S \rightarrow \infty)=1$ such that Eq. (3.14) and Eq. (3.16) are obtained. To generalise the granular buoyancy force an appropriate function $B(S)$ needs to be found. Two approaches are taken here that could approximate the behaviour of $B(S)$. First by using Voronoi tessellation in section 3.4.2 and secondly an approach that investigates structural changes (fabric) around the particle in section 3.4.3.

\subsubsection{VORONOI APPROACH}

A natural approach to satisfy both limits of the function $B(S)$ is to perform a volume correction of the particle as first proposed in Chapter 2 by defining

$$
B_{\phi}(S)=\frac{V_{V}}{V_{p}}
$$


where $V_{V}$ is the Voronoi volume of the intruder particle and $V_{p}$ is the particle volume. For a homogeneous mixture at $S=1$, each particle has (on average) identical values for $V_{V}$ and $V_{p}$ so Eq. (3.18) reduces to the bulk volume fraction $\phi_{b}$ satisfying Eq. (3.16). It can be shown that $B_{\phi}(S \rightarrow \infty)=1$. The Voronoi approach is therefore a simple and valid approach that defines the buoyancy force of objects within the two size ratio limits, based on purely geometrical arguments. This correction does not depend on the shape of the Voronoi volume, which could be measured [24]. The Voronoi approach does not guarantee physical correctness between the two limiting points as the particle is not (directly) aware of its Voronoi space and therefore a different approach based on contact forces is taken in the next section.

\subsubsection{SURFACE CONTACT DENSITY APPROACH}

As an alternative approach to $B_{\phi}(S)$, the structural changes around the intruder as function of $S$ are investigated. Although the interaction between particles is of a discrete nature, consider a situation where a simulation is in a steady state for a very long time such that a continuum approach is allowed. The time-averaged force on the intruder can then be expressed by

$$
\boldsymbol{F}_{\text {tot }}=\int_{A_{p}} \frac{d \boldsymbol{F}}{d A} d A
$$

where $d \boldsymbol{F}$ is time-average force on an infinitesimal surface element $d A$ of the sphere and $A_{p}$ is the surface area of the sphere. By taking a similar approach to Ref. [9], the force on the surface of the intruder can be split in a contact force component and a structural component. Applying the chain rule yields,

$$
\boldsymbol{F}_{\text {tot }}=\int_{A_{p}} \frac{d \boldsymbol{F}}{d C} \frac{d C}{d A} d A=\int_{A_{p}} \boldsymbol{F}_{c} E d A
$$

where $d C$ is the number of contacts on an infinitesimal surface element $d A$,

$$
\boldsymbol{F}_{c}:=\frac{d \boldsymbol{F}}{d C}
$$

is the (time-averaged) average contact force and

$$
E:=\frac{d C}{d A}
$$

is the (time-averaged) number of contacts per surface area, henceforth called the contact structure. Integrating $E$ over the surface area yields the coordination number $\mathcal{Z}$,

$$
\int_{A_{p}} E d A=\int_{A_{p}} \frac{d C}{d A} d A=\int_{0}^{\mathcal{Z}} d C=: \mathcal{Z} .
$$

\section{A. Normalisation}

For analysis purposes it is convenient to rewrite Eq. (3.20) by introducing normalised angular distribution functions. For the contact structure this distribution function is defined as

$$
P_{E}:=\frac{E A_{p}}{\mathcal{Z}}
$$


with the property

$$
\frac{1}{A_{p}} \int_{A_{p}} P_{E} d A=1
$$

Furthermore, the number of contacts per surface area, termed surface contact density, is defined as

$$
c_{\rho}:=Z / A_{p} .
$$

Taking Eq. (3.22) and substituting the definitions of Eq. (3.24) and Eq. (3.26) yields

$$
E=c_{\rho} P_{E}
$$

Similarly to the contact structure, the contact force is rewritten. First by defining a contact force magnitude $F_{c}$ and contact direction $\boldsymbol{n}_{c}$,

$$
\boldsymbol{F}_{c}:=F_{c} \boldsymbol{n}_{c}
$$

where $F_{c}$ is a scalar, but still is orientation dependent, $F_{c}=F_{c}(A)$. Normalisation of $F_{c}(A)$ is done by defining a normalised angular distribution function,

$$
P_{F_{c}}:=\frac{F_{c}(A)}{\bar{F}_{c}}
$$

where $\bar{F}_{c}$ is the average contact force magnitude (orientation-independent). The distribution function satisfies the property

$$
\frac{1}{A_{p}} \int_{A_{p}} P_{F_{c}} d A=1 .
$$

Combining Eq. (3.28) with Eq. (3.29) yields

$$
\boldsymbol{F}_{c}=\bar{F}_{c} P_{F_{c}} \boldsymbol{n}_{c} .
$$

Substituting Eq. (3.27) and Eq. (3.31) in Eq. (3.20) yields

$$
\boldsymbol{F}_{\text {tot }}=\bar{F}_{c} c_{\rho} \int_{A_{p}} P_{F_{c}} P_{E} \boldsymbol{n}_{c} d A .
$$

Note that both $\bar{F}_{c}$ and $c_{\rho}$ are orientation independent scalars, and only depend on S.

\section{B. FORCE DECOMPOSITION}

To define the granular buoyancy force, the total force $\boldsymbol{F}_{\text {tot }}$ needs to be split into a buoyant part and other remaining forces (such as lift and drag). Before splitting $\boldsymbol{F}_{\text {tot }}$ note that the contact structure $c_{\rho} P_{E}$ can not be split, because a contact can not be assigned to a single mechanism. Instead, the contact forces should be split into the various mechanisms. The split is performed by making an assumption about $\bar{F}_{c} P_{F_{c}}$.

The hydrostatic pressure in a granular flow stems from the contact forces between particles that support the weight of the flow. As the weight varies linearly in depth, the hydrostatic is also linear. It is therefore straightforward to assume that (i) the contact forces related to the hydrostatic pressure are also linear in depth. Moreover, the intruder 
size has no significant effect on the hydrostatic pressure which implies that (ii) the linear dependency is independent of $S$. Furthermore, (iii) the only force present in the hydrodynamic theory at $S=1$ is the buoyancy force, see section 3.4.1. With these three observations the split in the contact force is given by

$$
\bar{F}_{c}(S) P_{F_{c}}(S)=\bar{F}_{c}^{S=1} b_{z}^{S=1} z+R(S),
$$

where $\bar{F}_{c}^{S=1} b_{z}^{S=1}$ is the magnitude of the (i) linear, (ii) $S$-independent gradient of the contact forces at $S=1$. The second term $R(S)$ is the remainder of the contact forces, attributed to other forces. To accommodate observation (iii) it is mandatory for this split that integration of $R(S=1)$ yields no force, which will be verified in section 3.5.1. Combining the force decomposition of Eq. (3.33) with Eq. (3.32) leads to the following definition of the buoyancy force,

$$
F_{b}^{S}(S) \equiv \bar{F}_{c}^{S=1} c_{\rho}(S) \int_{A_{p}} b_{z}^{S=1} z P_{e}(S) \boldsymbol{n}_{c} \cdot \boldsymbol{e}_{z} d A .
$$

Note that although the contact forces are assumed independent of $S$, the buoyancy force could still depend on $S$ through the contact structure. The non-dimensionalised buoyancy for the surface contact density approach is then defined by

$$
B_{\rho}(S):=\frac{F_{b}^{S}}{F_{b}^{S \rightarrow \infty}},
$$

the ratio of Eq. (3.34) and Eq. (3.14). The assumptions made in the surface constant density buoyancy definition are verified in section 3.5 .2 by showing that $B_{\rho}(S)$ satisfies the correct physical limits.

\section{SuRface Contact Density}

By assuming that the contact forces attributed to the buoyancy do not depend on $S$, it directly implies that any change in $S$ comes from a change in contact structure. This can either be a change in $c_{\rho}$, angular distribution function, or the contact force direction $\boldsymbol{n}_{c}$. The impact of $c_{\rho}$ can be estimated by considering the contact between a bulk particle and the intruder. An area $A_{s}$ on the surface of the intruder is shielded from having contacts with any other bulk particles, see Refs. [25, 26]. In 3D $A_{s}$ is given by,

$$
A_{s}=2 \pi S^{2} r_{b}\left[1-\sqrt{1-\left(\frac{1}{S+1}\right)^{2}}\right],
$$

where the limits $A_{s}(S=1) / r_{b}^{2}=2 \pi(1-\sqrt{3 / 4}) \approx 0.84$ and $A_{s}(S \rightarrow \infty) / r_{b}^{2}=\pi$ show that $A_{s}$ depends on $S$. The surface contact density is closely related to the compacity, $c_{s}:=$ $\mathcal{Z} A_{s} / A_{p}$, which is the the ratio of the shielded area and the surface area of the intruder. Previous studies have assumed and validated this compacity to be approximately constant for larger particles in compressed polydisperse packings [25-27]. If the same assumption is used here, the surface contact density could be expressed as $c_{\rho}(S)=c_{s} / A_{s}(S)$ and therefore indicates that $c_{\rho}(S)$ could have a significant contribution the total force on the particle. The impact of $P_{E}$ is harder to estimate and will be investigated in section 3.5.1. 


\subsection{RESULTS}

To verify the surface contact density theory, both the structural fields $E$ and contact force fields $\boldsymbol{F}_{c}$ around the intruder are measured and analysed, using Eq. (3.1) and Eq. (3.5). The structure of these fields is investigated by fitting them, such that Eq. (3.35) can be measured. Analysis of these fields is in section 3.5.1. With these measurements the surface contact density theory can be validated which is done in section 3.5.2.

\subsubsection{ANGULAR DISTRIBUTION FUNCTIONS}

The contact force distribution $\hat{P}_{F_{c}}$ is shown in Fig. 3.2. Here the hat indicates a measured value of $P_{F_{c}}$ (see Eq. (3.29)) using Eq. (3.5). For $S=1$ the contact force distribution looks pill-shaped under an angle of $45^{\circ}$. As $S$ increases this shape changes and a clear asymmetry starts to develop, with a higher contact force at the bottom than at the top. A curious observation is that the angle of the anisotropy seems to be robust with a value of $45^{\circ}$. The contact force is fitted using the most important contribution from a second order Fourier series [12] and linear contributions in the $x$ - and $z$-direction ${ }^{1}$,

$$
\hat{P}_{F_{c}} \approx 1+b_{x} \cos (\theta) \sin (\phi)+b_{z} \cos (\phi)+a_{x z} \cos (\phi) \cos (\theta) \sin (\phi),
$$

where $a_{x z}$ is the most important contribution taking anisotropy into account at an angle of $45^{\circ}$ and $b_{x}$ and $b_{z}$ are the linear gradients. Although not present at $S=1, b_{x}$ emerges for increasing $S$. The fit is shown in Fig. 3.2 and shows very good agreement with the data. The coefficients as function of $S$ are shown in Fig. 3.3(a). A clear trend in all coefficients are shown. Coefficient $b_{x}$ does not seem to be present at $S=1$, but becomes important for $S>1$. The coefficient $b_{z}$ at $S=1$ corresponds to the buoyancy force and is clearly present. Curiously, even this coefficient increases for increasing $S$, which could be related to the presence of lift and drag forces. The anisotropy increases which can also be clearly observed in Fig. 3.2.

In Fig. 3.4 $\hat{P}_{E}=\left(\hat{E} A_{p}\right) / Z$ is shown for various values of $S$. The normalisation ensures that the profiles can be compared directly. All situations are dominated by the shear flow where the top-left and bottom-right part of the intruder encounter many flow particles. The top-right and bottom-left barely show any contact. Specifically for $S=1$ a horizontal contact plane can be observed, and a contact plane under an angle of roughly $25^{\circ}$. This might be caused by the structural shielding surface area by the horizontal plane particles. It is not observed for $S>1$. As $S$ increases an interesting asymmetry in the $z$-direction can be observed. Especially for $S=3.2$ the top-left has more contacts than the bottomright.

Further investigation of the structure is done by proposing a fit function. Here we use a similar fit function as used for $\hat{P}_{F_{c}}$, normalised by the particle surface area,

$$
\hat{P}_{E} \approx \frac{1}{4 \pi r_{p}^{2}}\left(1+a_{x z}^{E} \cos (\phi) \cos (\theta) \sin (\phi)+b_{x}^{E} \cos (\theta)+\sin (\phi) b_{z}^{E} \cos (\phi)\right) .
$$

The fitted surfaces shown in Fig. 3.4 seem to capture most trends, except close to $S=1$. The coefficients as a function of $S$ are shown in Fig. 3.3(b). The value of $a_{x z}^{E}$ first shows

${ }^{1}$ This series can also be expressed in spherical harmonics as $\hat{P}_{F_{c}} \approx 1+c_{1}^{1} \operatorname{Re}\left(Y_{1}^{1}\right)+c_{1}^{0} \operatorname{Re}\left(Y_{1}^{0}\right)+c_{2}^{1} \operatorname{Re}\left(Y_{2}^{1}\right)$ with $c_{1}^{1}=-2 \sqrt{2 \pi / 3} b_{x}, c_{1}^{0}=2 \sqrt{\pi / 3} b_{z}$ and $c_{2}^{1}=-2 \sqrt{2 \pi / 15} a_{x z}$. 
$\hat{P}_{F_{c}}$ in $x z$-plane view

Fit of $\hat{P}_{F_{c}}$

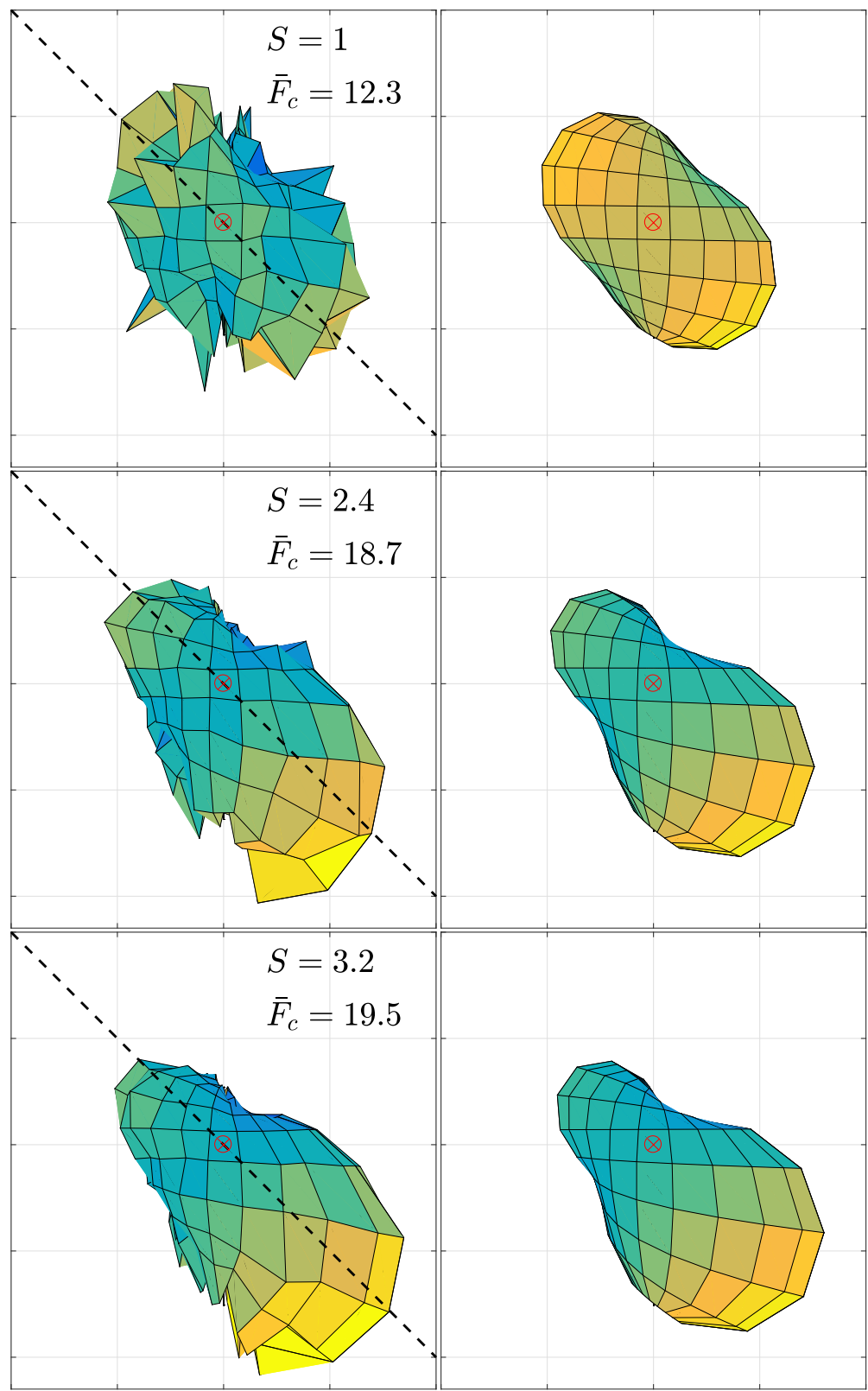

Figure 3.2: In the left panel the normalised contact force distribution around an intruder for increasing $S$ on equal grids and in the $x z$-plane. The red cross marks the center of the plot. Dashed lines are visual guides. In the left panels fits of the measured data to Eq. (3.37) are shown. The color bar for all plots are identical. 


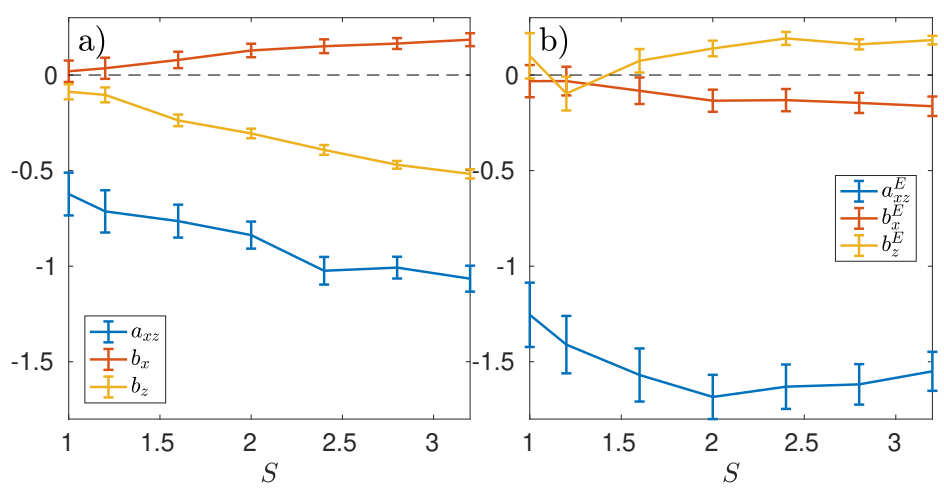

Figure 3.3: Coefficients obtained from fitting a) $\hat{P_{F_{c}}}$ to Eq. (3.37) and b) $\hat{P_{E}}$ from fitting Eq. (3.38). The error bars indicate the $95 \%$ confidence intervals of the fitting parameter.

a decrease, to the point of $S \approx 2$ and then increases again slightly. The linear trend observed in Fig. 3.4 is both captured by $b_{x}^{E}$ and $b_{z}^{E}$, although $b_{z}^{E}$ seems to be more dominant.

\subsubsection{VALIDATION}

Validation of the fit functions is done by computing the total force using Eq. (3.6) in combination with the fit functions Eq. (3.38) and Eq. (3.37). Comparison of this with the measured total force is shown in Fig. 3.5(a). The fit functions capture the global trend, yet significant deviations can be observed, especially for values close to $S=1$. This is no surprise when comparing the fit to the measurements in Fig. 3.4. When the raw data for $P_{E}$ is used, the blue curve is obtained, which is a very good match. This indicates that $P_{F_{c}}$ is captured really well by the fit function.

Validation of the surface contact density theory is done by measuring Eq. (3.35). To stay close to the true nature of the measurement, only $P_{F_{c}}$ is fitted to create a clean split between the various terms. The structure $P_{E}$ and contact direction $\boldsymbol{n}_{c}$ are used straight from measurements which yield better results in light of Fig. 3.5(a). This is important, because the force gradient in the flow is measured at $S=1$.

The scaled buoyancy force is shown in Fig. 3.5(b) and at $S=1$ it has a value of roughly $1.05 / \phi$, which is equal to the total force measured in DPM at $S=1$. This force is purely produced by the linear slope encountered in the contact force, as the other components do not contribute. For increasing $S$ the buoyancy force reduces slowly and seemingly converges towards 1 , as measured in experiments. The surface contact density and nondimensional buoyancy force are proportional to each other, $B_{\rho} \propto c_{\rho}$, with a proportionality constant of 2.2. Therefore it can be concluded that the physical mechanism changing the buoyancy force is the surface contact density.

The Voronoi approach $B_{\phi}$ is also shown in Fig. 3.5(b). When comparing both $B_{\phi}$ and $B_{\rho}$, it can be seen that the Voronoi over estimates the buoyancy force for larger $S$, while the surface contact density approach under estimates this. The deviations however stay 
$\hat{P}_{E}$ in $x z$-plane view
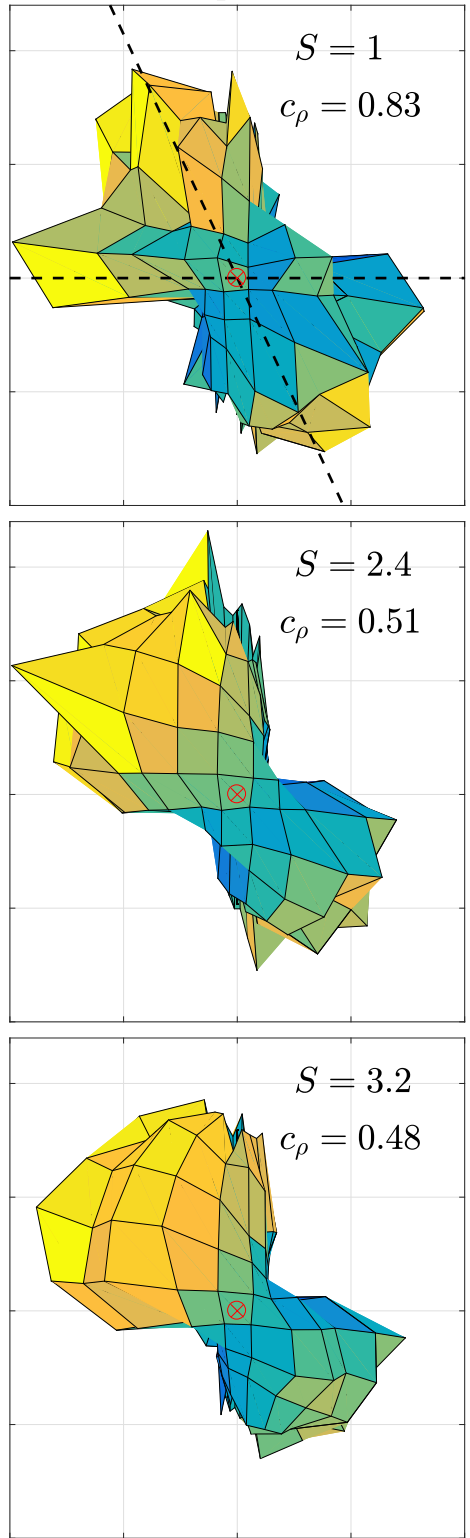

Fit of $\hat{P}_{E}$
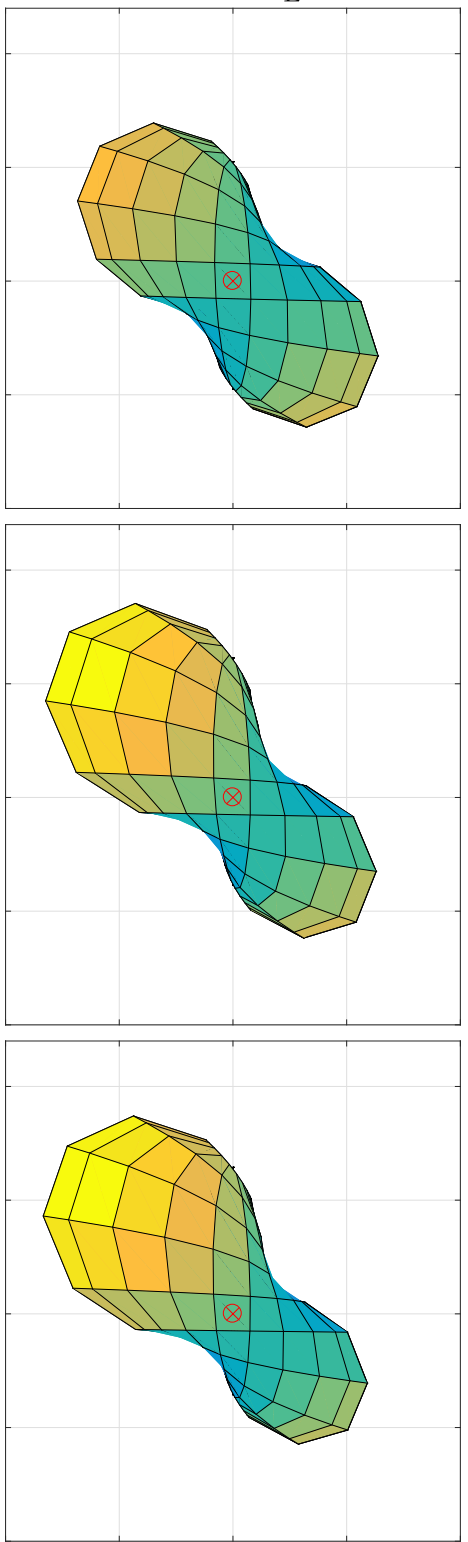

Figure 3.4: The angular distribution $\hat{P}_{E}$ of the number of contacts per surface area is shown for different values of $S$ in the left panels. The scale is identical for all plots and the plane of view is the $x z$-plane. The red cross marks the center of the plot. The black dashed lines are visual indicators. In the right panels the fit surface of the measurements to Eq. (3.38) are shown. 

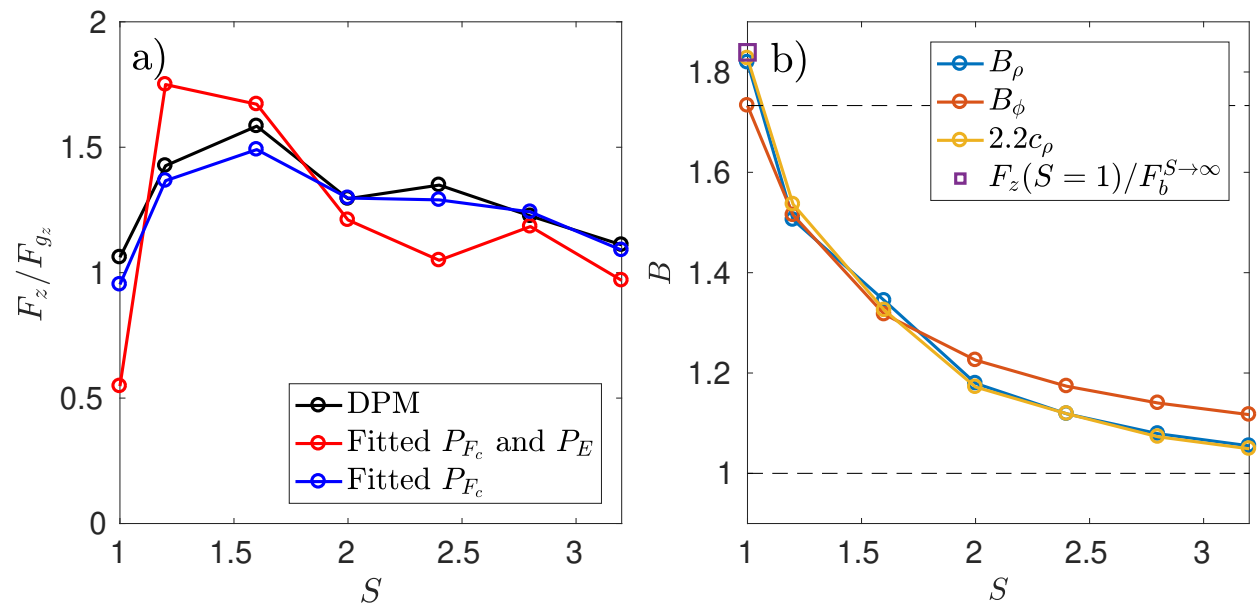

Figure 3.5: a) Total force on the intruder as size ratio changes. Fit functions for $P_{F_{c}}$ and $P_{E}$ are Eq. (3.37) and Eq. (3.38), respectively. The blue line represents a mixed case where the raw measurements are used for $P_{E}$. b) Non-dimensional buoyancy force. The dashed lines correspond to the two physical limits of $B$ with $1 / \phi_{b}$ and 1. The green square indicates the measured total vertical force from DPM measurements at $S-1$

within $10 \%$ of $B_{\rho}$ which is acceptable from a modeling perspective.

In practice, the Voronoi approach is easy to measure compared to the surface contact density approach. This is because the latter approach requires a measurement of the contact force profile at $S=1$ and measuring the coordination number requires more time snaps due to its discrete nature. Furthermore, the Voronoi approach naturally satisfies both theoretical limits of $S=1$ and $S \rightarrow \infty$. Between these two points the Voronoi slightly over estimates the buoyancy force, but is close enough to the physical mechanism.

\subsection{CONCLUSION \& DisCUSSION}

In this work we derive a micro mechanical definition of the buoyancy force and compare it to the recently proposed buoyancy force in [8]. Both approaches yield very similar result, revealing a microscopic explanation for the observed deviation from Archimedes' principle. As the intruder increases in size, the apparent curvature of the intruder reduces, leading to a decrease in contacts per intruder surface area. The physical explanation of the surface contact density approach gives a solid base for the generalised Archimedes principle for spherical objects in granular flow, but the Voronoi approach seems more practical in use as it does not require to fit contact forces at $S=1$. Furthermore, this mechanism is relevant for all the contact forces, not only the buoyancy force. This is an important realisation when developing a force model for a single particle in granular flows, as the gravity force is the only force that does not depend on this mechanism.

The buoyancy force for $S<1$ has not been investigated and is future work. It is ex- 
pected that the definition still holds true for values slightly smaller than one, but at some point will deviate significantly as the structure will change significantly when intruders start to percolate. Obtaining good statistical data for $f$ and $\boldsymbol{n}_{\boldsymbol{c}}$ around small particles is computationally expensive as the number of contacts on these particles is small. However, when good statistical data is obtained, numerical integration can still be done to investigate the contact force and structural fields.

Last, but not least, a philosophical question can be asked: When is the intruder part of the fluid, and when does it become an object inside the fluid? From Fig. 3.5(b) it appears that for $S>3.2$ there is barely any change in either $F_{b}$ or $F_{L}$. This coincidentally agrees with observations for particles that start to sink instead of segregate [28].

\section{REFERENCES}

[1] J. Guo, Motion of spheres falling through fluids, Journal of Hydraulic Research 49, 32 (2011).

[2] A. Tripathi and D. V. Khakhar, Numerical simulation of the sedimentation of a sphere in a sheared granular fluid: a granular stokes experiment, Physical Review Letters 107, 108001 (2011).

[3] S. Liu and J. J. McCarthy, Transport analogy for segregation and granular rheology, Physical Review E 96, 020901 (2017).

[4] F. Guillard, Y. Forterre, and O. Pouliquen, Scaling laws for segregation forces in dense sheared granular flows, Journal of Fluid Mechanics 807 (2016).

[5] K. Nichol, A. Zanin, R. Bastien, E. Wandersman, and M. van Hecke, Flow-induced agitations create a granular fluid, Physical Review Letters 104, 078302 (2010).

[6] D. A. Huerta, V. Sosa, M. C. Vargas, and J. C. Ruiz-Suárez, Archimedes' principle in fluidized granular systems, Physical Review E 72, 031307 (2005).

[7] R. Piazza, S. Buzzaccaro, E. Secchi, and A. Parola, What buoyancy really is. a generalized archimedes' principle for sedimentation and ultracentrifugation, Soft Matter 8, 7112 (2012).

[8] K. van der Vaart, M. P. van Schrojenstein Lantman, T. Weinhart, S. Luding, C. Ancey, and A. R. Thornton, Segregation of large particles in dense granular flows suggests a granular saffman effect, Physical Review Fluids 3, 074303 (2018).

[9] L. Rothenburg and R. Bathurst, Analytical study of induced anisotropy in idealized granular materials, Geotechnique 39, 601 (1989).

[10] F. Radjai, D. E. Wolf, M. Jean, and J.-J. Moreau, Bimodal character of stress transmission in granular packings, Physical Review Letters 80, 61 (1998).

[11] N. P. Kruyt and L. Rothenburg, Kinematic and static assumptions for homogenization in micromechanics of granular materials, Mechanics of Materials 36, 1157 (2004). 
[12] H. Ouadfel and L. Rothenburg, Stress-force-fabric relationship for assemblies of ellipsoids, Mechanics of Materials 33, 201 (2001).

[13] L. E. Silbert, D. Ertaş, G. S. Grest, T. C. Halsey, D. Levine, and S. J. Plimpton, Granular flow down an inclined plane: Bagnold scaling and rheology, Physical Review E 64, 051302 (2001).

[14] T. Weinhart, A. R. Thornton, S. Luding, and O. Bokhove, Closure relations for shallow granular flows from particle simulations, Granular Matter 14, 531 (2012).

[15] T. Weinhart, R. Hartkamp, A. R. Thornton, and S. Luding, Coarse-grained local and objective continuum description of three-dimensional granular flows down an inclined surface, Physics of Fluids 25, 070605 (2013).

[16] P. A. Cundall and O. D. L. Strack, A discrete numerical model for granular assemblies, Geotechnique 29, 47 (1979).

[17] S. Luding, Cohesive, frictional powders: contact models for tension, Granular matter 10, 235 (2008).

[18] A. R. Thornton, T. Weinhart, V. Ogarko, and S. Luding, Multi-scale methods for multi-component granular materials, Computational Methods in Materials Science 13 (2013).

[19] T. Weinhart, D. R. Tunuguntla, M. P. van Schrojenstein-Lantman, A. J. van der Horn, I. F. C. Denissen, C. R. Windows-Yule, A. C. de Jong, and A. R. Thornton, Mercurydpm: A fast and flexible particle solver part a: Technical advances, in Proceedings of the 7th International Conference on Discrete Element Methods (Springer, 2017) pp. 1353-1360.

[20] I. Goldhirsch, Stress, stress asymmetry and couple stress: from discrete particles to continuous fields, Granular Matter 12, 239 (2010).

[21] T. Weinhart, A. R. Thornton, S. Luding, and O. Bokhove, From discrete particles to continuum fields near a boundary, Granular Matter 14, 289 (2012).

[22] P.-Y. Lagrée, L. Staron, and S. Popinet, The granular column collapse as a continuum: validity of a two-dimensional navier-stokes model with a $\mu(I)$-rheology, Journal of Fluid Mechanics 686, 378-408 (2011).

[23] A. Tripathi and D. V. Khakhar, Density difference-driven segregation in a dense granular flow, Journal of Fluid Mechanics 717, 643-669 (2013).

[24] A. Sufian, A. R. Russell, and A. J. Whittle, Evolving pore orientation, shape and size in sheared granular assemblies, Granular Matter 21, 4 (2018).

[25] M. R. Shaebani, M. Madadi, S. Luding, and D. E. Wolf, Influence of polydispersity on micromechanics of granular materials, Physical Review E 85, 011301 (2012). 
[26] M. Madadi, O. Tsoungui, M. Lätzel, and S. Luding, On the fabric tensor of polydisperse granular materials in 2d, International Journal of Solids and Structures 41, 2563 (2004).

[27] F. Göncü, O. Durán, and S. Luding, Constitutive relations for the isotropic deformation of frictionless packings of polydisperse spheres, Comptes Rendus Mécanique 338, 570 (2010), Micromechanics of Granular Materials.

[28] N. Thomas and U. D'ortona, Evidence of reverse and intermediate size segregation in dry granular flows down a rough incline, Physical Review E 97, 022903 (2018). 



\section{4}

\section{High Resolution CONTINUUM FiELDS AROUND A SPHERICAL INTRUder IN A CHUTE FlOW}

This work presents high-fidelity continuum fields around a single large intruder particle in a dense, steady mono-disperse granular chute flow. The density, velocity and stress fields are obtained using the coarse graining technique converting discrete particle data into continuum fields compatible with the conservation laws of mass and momentum. Size, density and friction of the intruder are changed to observe the response of the granular flow. Clear layering structures, size and friction dominated velocity profiles and rich non-Newtonian stress effects are observed in the vicinity of the intruder. Possible mechanisms for segregation have been identified that could help develop a more fundamental understanding of segregation due to size, density and friction, eventually leading to better predictions of segregation. The investigated continuum fields could inspire the development of new constitutive models for granular flows, and contribute to the development of continuum or up-scaled simulation techniques better capable of simulating objects in realistic dense granular flows with too many particles.

\subsection{INTRODUCTION}

Granular flows are important in industrial environments such as the food, pharmaceutical and agricultural industries, and in the geophysical context with pyroclastic flows and avalanches or land slides as examples. The flow of these granular materials exhibit many different complex phenomena such as dilatancy [1], anisotropy [2, 3], shear bands [4, 5] and segregation [6-8]. Segregation in dense granular flows is a persistent phenomenon that induces various flow features such as fingering [9], bulbous head formation [10] and generally the de-mixing of mixed flows [6]. However, the fundamental details of the cause of segregation are still not fully understood.

Considering a granular flow with identical spherical particles, no segregation takes 
place as the particles have no preferred position in the flow. Yet, when one of the particles changes its properties, that particle usually starts to segregate. The particle size is widely regarded as the most important property affecting segregation. It is therefore the most studied particle property, especially with the focus on 50/50 volume ratio mixtures [11-15], for which segregation models have been successfully developed [16-18]. However, industrial applications often tend to have more dilute volume ratio mixtures, such as 10/90 mixtures. The models developed for 50/50 mixtures don't capture the dilute regimes as accurately [19]. Furthermore, reverse segregation is observed for very large particles [20] which is not included in the current models either. Investigations of polydisperse systems with large size distributions have also been investigated previously in Refs. [21, 22] and references therein.

Density differences in mixtures also induce segregation [23] and have been studied in a chute flow setup [24]. The resulting model was inspired by the discovery of a (modified) Stokes drag law on a single intruder particle $[25,26]$. Improved segregation models have take both size and density into account $[27,28]$. The effect of friction on segregation has had less attention than density and size [29, 30], but could have significant effects too [31].

To improve the fundamental understanding of segregation, single intruders have been investigated. The segregation force was found to be related to the derivative of pressure and shear stress [32]. Recently a connection between a horizontal velocity lag and the segregation force was found [33]. The importance of rotation of an intruder particle has also been linked to size segregation [34]. These results are pieces of a complex puzzle, but the true origin of size segregation has yet to be revealed.

Thus far, these investigations have focussed on the forces on the intruder itself, yet the reverse effect of the intruder particle on the flow has not yet been investigated and could yield valuable insights into the mechanics of granular flows and segregation. In this work we focus on the effect of size, density and friction of the intruder on the flow. The flow around the intruder is visualised by converting long time-averaged particle data into continuum fields using the coarse graining method [2, 35, 36]. By comparing high resolution density, velocity and stress fields for different intruder properties, we aim to obtain more insight in the processes and mechanisms that cause segregation.

First the simulation and coarse grain methods are discussed in section 4.2. The structure, velocity and stress fields are then discussed in section $4.3,4.4$ and 4.5 , respectively. Based on the investigations of the fields, conclusions are draw in section 4.6.

\subsection{Simulation And Methods}

Gravity-driven granular flow down an incline (chute flow) is a well studied system, see Refs. $[2,37,38]$ and references therein. In this work we chose a similar setup as it produces a velocity field which is shearing in only one plane. Discrete particle method (DPM) simulations of a chute flow are performed by the open-source software package MercuryDPM (MercuryDPM.org; [39, 40]).

A schematic description of the simulation setup is shown in Fig. 4.1(a). A chute angle of $\theta_{c}=22^{\circ}$ is used and periodic boundaries are applied in the $x$ - and $y$-directions. The system is non-dimensionalised such that the non-dimensional bulk particle diameter is $d_{b}=1$, density $\rho_{b}=6 / \pi$ and the gravitational acceleration $g=1$. The gravity vec- 
tor is given by $\boldsymbol{g}=g\left[\sin \left(\theta_{c}\right), 0,-\cos \left(\theta_{c}\right)\right]$. The simulation box has a size $[L x, L y, L z]=$ $[20,8.9,40] d_{b}$. Within this box roughly 6000 dry frictional particles are simulated yielding an average flow height of $h \approx 30 d_{b}$. A rough bottom is composed of (slightly) larger than bulk particles, with a diameter of $1.7 d_{b}$. Details for the method of creating the rough bottom can be found in Ref. [38].

Contacts between particles are modeled using a linear spring-dashpot model [2, 41] with linear elastic and linear dissipative contributions for the normal and tangential forces between particles. Coulomb friction in the tangential direction is modeled by a slider. Particle properties and contact parameters are given in Table 4.1. The stiffness and dissipation of the contact laws are computed through the analysis of two colliding particles [42]. The tangential, sliding and rolling friction between bulk particles are all equal to the friction coefficient $\mu_{b b}$ in Table 4.1. The friction between intruder and bulk particles is given by $\mu_{p b}$ and is varied.

An intruder with size ratio $S$ is introduced in the flow at a height of $z_{p, 0}=23$ and to keep the intruder at approximately the same height, a restoring spring is introduced [32] in the $z$-direction, while it can freely flow in the $x$ - and $y$-directions. The spring stiffness of the restoring spring is set to $k_{s}=20$. The non-dimensional intruder size $S$, density $D$ and friction $M$ are scaled such that unity implies the value of a bulk particle, see Table 4.1 .

\begin{tabular}{|l|l|l|}
\hline Parameter & Symbol & value \\
\hline bulk particle diameter & $d_{b}$ & 1.0 \\
particle density & $\rho_{b}$ & $\pi / 6$ \\
\hline Restitution coefficient & $e_{r}$ & 0.1 \\
Contact duration & $t_{c}$ & 0.005 \\
Contact friction & $\mu_{b b}$ & 0.5 \\
\hline Size ratio & $S=d_{p} / d_{b}$ & $1.0-3.2$ \\
Density ratio & $D=\rho_{p} / \rho_{b}$ & $1 / 6-10 / 6$ \\
Friction ratio & $M=\mu_{p b} / \mu_{b b}$ & $1.0 \times 10^{-4}-1.0 \times 10^{4}$ \\
\hline
\end{tabular}

Table 4.1: Dimensionless particle properties and contact model parameters. Contact duration and restitution coefficient set the stiffness and dissipation of the particles, see Ref [42]. The subscript $p$ indicates an intruder property.

An important part of the analysis of this research is the continuum density profile around the intruder particle. To obtain this field a technique called coarse gaining (CG) is employed [2, 35, 36]. This technique takes the micromechanical information of the granular flow and transforms it into a macromechanical field-based description. As example, the "micro" point-density of the centers of mass is

$$
\rho^{\mathrm{mic}}(\mathbf{x}, t)=\sum_{i=1}^{N} m_{i} \delta\left(\mathbf{x}-\mathbf{x}_{i}(t)\right),
$$

where $\mathbf{x}_{i}$ is the position of particle $i, m_{i}$ is its mass and $N$ the total number of particles. Transforming this to a macromechanical field is done by replacing the delta function by 
a smoothing kernel $\mathbb{W}\left(\boldsymbol{x}-\boldsymbol{x}_{i}\right)$ with $\int \mathscr{W}\left(\boldsymbol{x}-\boldsymbol{x}_{i}\right) d \boldsymbol{x}=1$, so that

$$
\rho(\mathbf{x}, t)=\sum_{i=1}^{N} m_{i} \mathscr{W}\left(\mathbf{x}-\mathbf{x}_{i}\right),
$$

Other continuum fields such as momentum and stress can be treated in a similar manner, for full details see Ref. [2]. Possible choices for the smoothing kernel are a Gaussian function, Lucy polynomial [43] or a Heaviside function. Regardless of which function is taken, a coarse graining length scale $w$ has to be chosen that accurately catches the problem. In this work a Heaviside smoothing kernel is used,

$$
\mathscr{W}(\mathbf{x})=\left\{\begin{array}{ll}
\frac{1}{(4 / 3) \pi w^{3}} & |\mathbf{x}| \leq w \\
0 & |\mathbf{x}|>w
\end{array},\right.
$$

with a smoothing length scale $w$ the size of the granular fluid particles radius, $w=d_{b} / 2$. The length scale is chosen such that for a given time, the instantaneous density field accurately matches the density fields of the bulk particles. Furthermore, this gives a sharp interface at the surface of the intruder. A larger width could smooth out the layering structure around the intruder.

Evaluation of the density field on a spherical surface is done using the quadrature points of the Lebedev quadrature [44]. These points are distributed such that no bias with the angle is generated on the sphere. The average value of a field $f$ in a shell around the intruder surface is defined by

$$
\bar{f}=\frac{1}{V_{s}} \int_{r_{p}}^{r_{s}} \int_{A_{L}} f d A d r,
$$

where $V_{s}$ is the volume of the shell and $r_{s}$ is the outer radius of the shell. Here $r_{s}=$ $r_{p}+2 r_{b}$ if not stated otherwise. Numerical integration is done by performing a combined trapezoidal rule in the radial direction and discretisation by the Lebedev quadrature in the azimuthal and polar directions.

\subsection{STRUCTURE}

The structure around the intruder is investigated by analysing the density profiles. A schematic situation of the intruder in the $x z$-plane is shown in Fig. 4.1(b). The intruder experiences the shear rate $\gamma_{x z}$ of the bulk flow. The velocity of bulk particles are denoted by the red arrows. The shear rate introduces compression in the top-left and bottomright corner. In these regions bulk particles have to move around the intruder. In the top-right and bottom-left corner tension regions emerge. In these regions bulk particles gain some freedom as they just have overtaken the intruder, therefore they will be less likely to stay attached to the surface of the intruder (like separation in fluids). The effect of compression and tension caused by the shear will have effect on the structure around the intruder. How this structure changes based on size ratio, density ratio and friction ratio is investigated in the next sections. 
(a) Chute flow setup

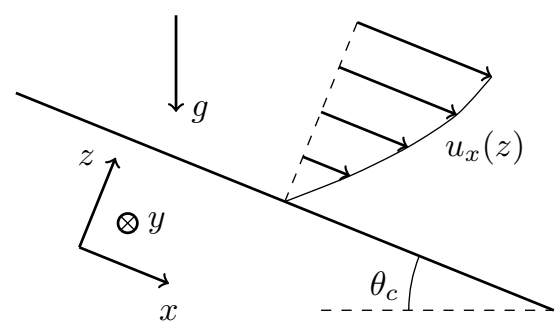

(b) Local system around an intruder

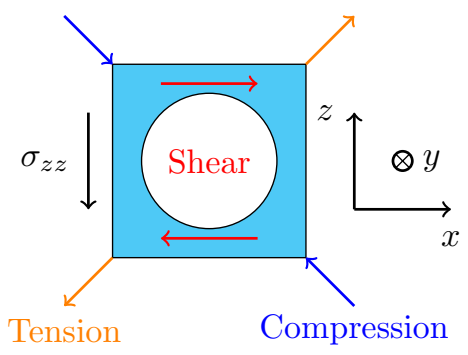

Figure 4.1: (a) Schematic of a chute flow down an incline of $\theta_{c}=22^{\circ}$. The gravity force $g$ induces a velocity profile $u_{x}(z)$. (b) Schematic of the intruder situation in the $x z$-plane, with the $y$-direction perpendicular into the plane. The shear introduces compression and tension at different regions on the particle.

\subsubsection{DENSITY FIELD}

The density field for various $S$ is shown in Fig. 4.2. An (almost) spherical layer is visible directly around an intruder of $S=1$ (no size-effect). A second (almost) spherical layer is present as well, however in diminished strength. A third layer and fourth layer can also be observed, although they have a relative straight structure. This indicates that the small particle only has a range of influence of approximately 2 particle layers. A remarkable observation is the general formation of (straight) layers in the bulk flow, far away from the intruder. The formation of layers seem to be a robust feature that even remain in non-planar flows [45].

A similar structure is observed for increasing size ratio, although the third and fourth layers become more pronounced. This implies that the interaction distance of the large particle increases as $S$ increases. For the case of $S=2.4$ more clear layering can be observed left of the intruder compared to the right side. This could be caused by the observation that the intruder is moving slower than the average flow, see Chapter 2.

The layers in the $z$-direction are shown in more detail in Fig. 4.3, where the coordinate $z-\left(z_{p}+r_{p}\right)$ allows for direct comparison between the different intruder sizes. The first minimum is at approximately $z-\left(z_{p}+r_{p}\right)=1$ as the bulk particles have a diameter of one and can not penetrate the intruder. For larger values, the distance between peaks stays approximately constant with an average distance of approximately 0.85 which is close to the measured value of 0.9 at the bottom of chute flows [2]. As the size ratio increases, the peak values of the layers seem to increase. The minima between the layers show no significant trend.

\section{FIRST LAYER FOR DIFFERENT SIZE RATIO}

A high resolution density field of the first layer around an intruder of $S=1$ is shown in Fig. 4.4(a), as reference situation, revealing a few interesting effects. Firstly, a layer of particles is observed around the intruder, with six strong maxima. Due to the coarse graining method it is expected that the peak values correspond to the preferred centre 

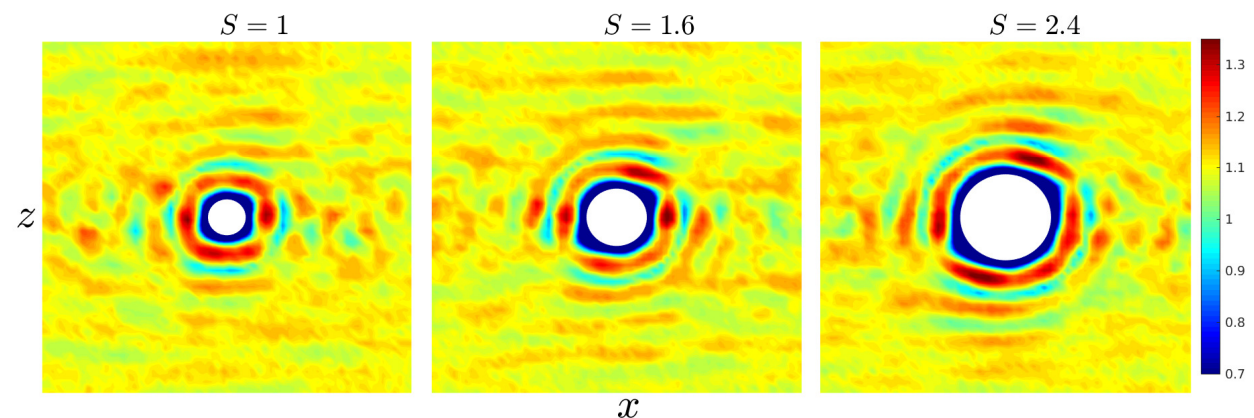

Figure 4.2: Coarse grained density far-field around an intruder particle in the $x z$-plane with coarse grain width of $w=0.5$ for $S=\{1.0,1.6,2.4\}$.

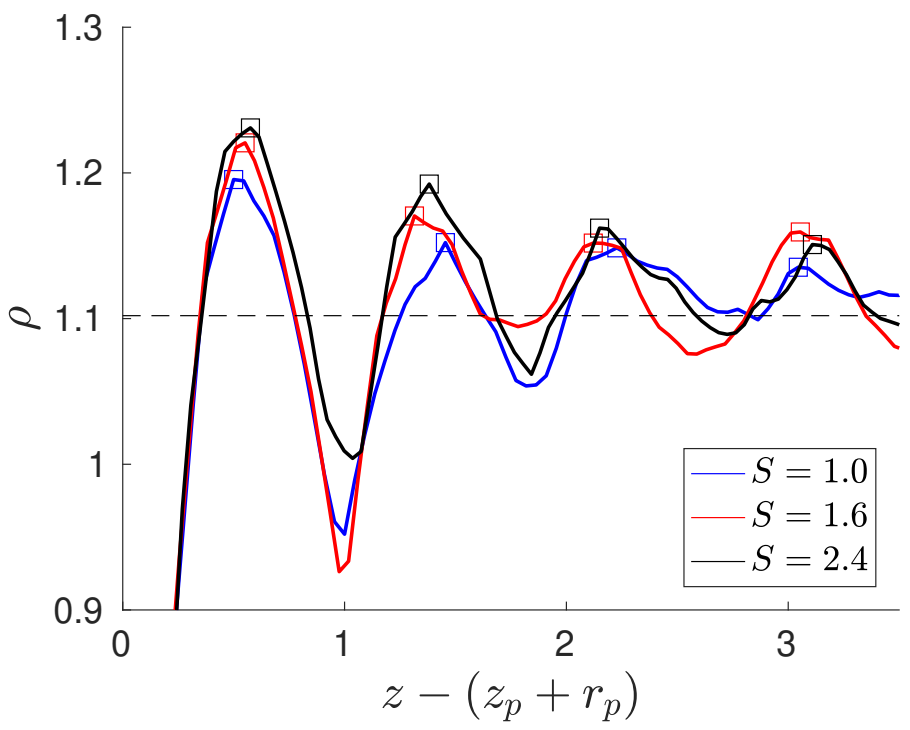

Figure 4.3: Layer density in the upward direction from the surface of an intruder particle for different sizes. The radius is subtracted from $z$ for direct comparison between different $S$. The dashed line indicates the average density in the flow. 

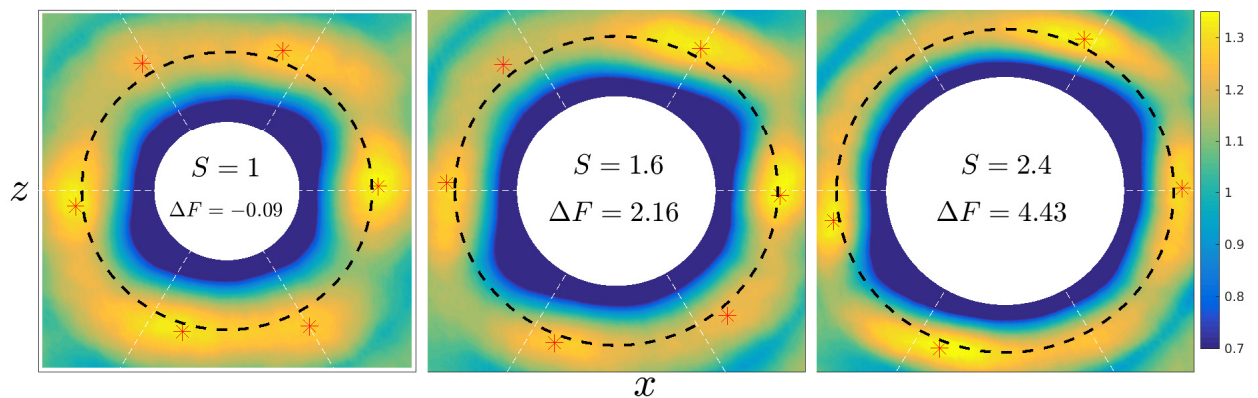

Figure 4.4: Coarse grained density profiles around an intruder at $z_{p, 0}=23$ in the $x z$-plane with coarse grain width of $w=0.5$. The focus is on the density field between the surface of the particle and the first layer. The $x$ and $z$-axis limits are therefore defined as $\left[-\left(0.8+d_{p} / 2\right),\left(0.8+d_{p} / 2\right)\right]$. White dashed lines indicate $0^{\circ}, 60^{\circ}$ and $120^{\circ}$ angles. The dashed black line refers to the center of the closest possible fluid particle with a distance of $d_{b}$ to the surface of the particle. Local maximum values in the density profile are marked by $*$.

positions of the particles in the first layer. The closest possible positions of rigid particles around the intruder particle are depicted by the dashed black line. All maxima are slightly outside this black line, because there is no configuration where particles are in permanent contact with the intruder. A slight separation of roughly $0.025 d_{b}$ is measured, where $d_{b}$ is the diameter of the fluid particle. The density at the surface of the intruder particle is practically zero due to the CG width of $w=0.5$. Deviations of zero are purely caused by the tiny overlaps between intruder and bulk particles during contacts.

Strong variations of density can be observed in the layer around the intruder. In general, when a fluid moves around a spherical object, stagnation points are expected where the relative fluid velocity is zero. In the current flow situation this would imply stagnation points left and right of the intruder. The maxima left and right of the intruder correspond to such a points, however in case of a granular flow they become stagnation zones due to the size of fluid particles. Due to the presence of a particle at the stagnation zones left and right of the intruder, there is reasonable part of the intruder shielded from possible contact. The next possible position of a particle would be at an angle of 60 degrees from the horizontal axes. The two maxima at the top-right and bottom-left do not emerge as clearly as the maxima left and right of the particle. Less strong, but also present are maxima at the top-left and bottom-right corner. The compressional and tensional regions, as depicted in the schematic situation of the intruder, see Fig. 4.1(b), clearly emerge in the density profile. The layer of particles stays attached to the intruder in the compressional region, while in the tensional region the flow shows detachment from the intruder.

For $S>1$, the structure of the density profile does not change much. The features merely get better defined. Although, the maxima in the top-left and bottom-right seem to disappear. In general for increasing $S$ the attachment in the compression area and detachment in the tension area become more pronounced. A 3D surface of the density profile of the first layer for $S=2.4$ is show in Fig. 4.5. The compression region and detachment regions are clearly visible and the layer seems quite symmetric in the $y z$-plane. 


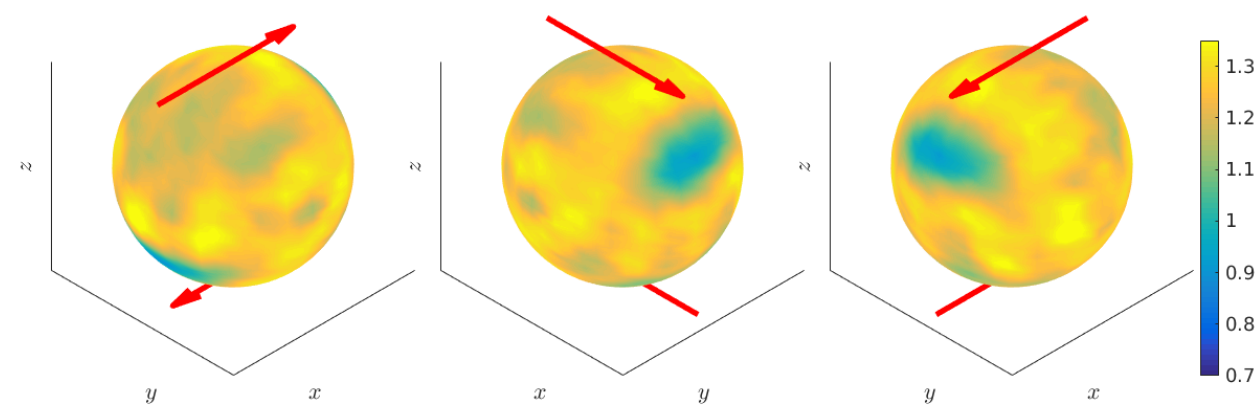

Figure 4.5: Coarse grained density field with $w=0.5$ around an intruder particle of $S=2.4$ at a distance of $r_{p}+r_{b}$, depicting the density fluctuations of first layer of particles around the intruder with the same colour bar as Fig. 4.4. The average density in the flow is 1.1. The points of view are, respectively, $\boldsymbol{x}=\{-1,-1,1\}$, $\boldsymbol{x}=\{1,-1,1\}$ and $\boldsymbol{x}=\{1,1,1\}$. The arrows indicate the shear direction.

\section{FIRST LAYER FOR DIFFERENT DENSITY RATIO}

The density profiles around the intruder particle for changing density ratio $D$ are shown in Fig. 4.6. The structure around the intruder is still dominated by compression and tension regions as schematically shown in Fig. 4.1(b). However, a distinct new feature emerges as $D$ changes. The stagnation regions shift as the particle changes density. The stagnation zones left and right of a light intruder move towards the compression region, reducing the effective compression region. The opposite effect is happening for dense intruders. Additionally one of the stagnation regions diminishes in magnitude. For an intruder with a velocity similar to the bulk flow, the stagnation zones are equally strong and symmetric around the $z$-axis. A possible explanation for the change in stagnation region intensity could be that as the density of the intruder changes, the gravity force is increased, yielding a different velocity lag [33] in the $x$-direction and hence the flow profile around the particle changes. Variation of $D$ shown here remains close to the original density and it is expected that a very heavy particle would eventually plough through the granular flow. This should yield a compression zone at the front of the intruder, and a wake behind the intruder. The transition to this situation can already be observed for $D=10 / 6$. The opposite is happening for $D \rightarrow 0$ as the particle then lags behind the flow and creates a more intense stagnation region zone on the left side, and a wake on the right side as can be observed at $D=1 / 6$.

\section{FIRST LAYER FOR DIFFERENT FRICTION RATIO}

Similar to $S$ and $D$, the density profiles for various friction ratio $M$ are shown in Fig. 4.7. Although the fields do seem to change with different $M$, no clear trend can be observed.

\subsubsection{AVERAGED LAYER DENSITY}

A measure to more quantitatively observe changes in the first layer around the intruder is the averaged layer density $\rho_{L}$. The layer density is computed using Eq. (4.4) with $r_{s}=$ $r_{p}+2 r_{b}$ to capture the first layer. In Fig 4.8(a) the layer density is shown as function of $S$. A clear downwards trend can be observed as the size ratio increases. It is expected that as $S \rightarrow 0$ the average density of the flow, $\rho_{L}=\rho_{b} \phi$ is obtained, since it would be 

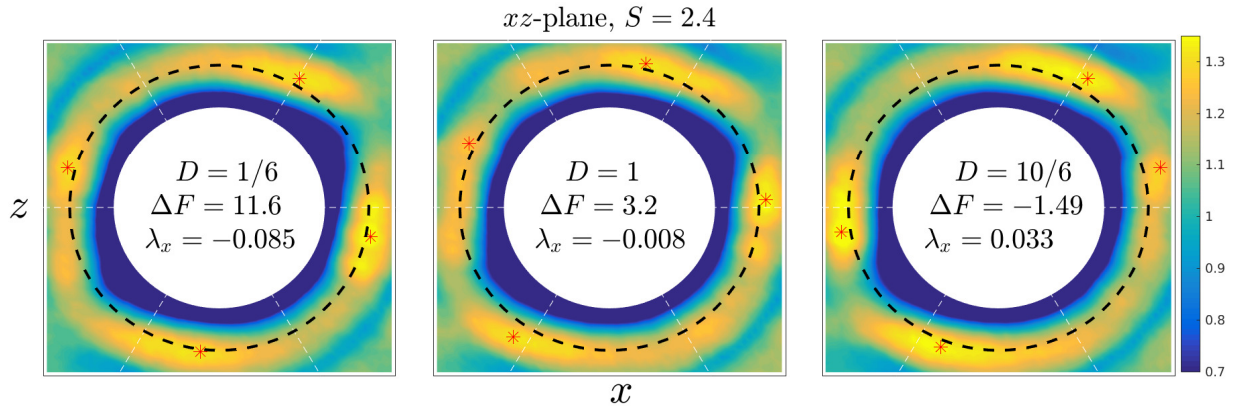

Figure 4.6: Coarse grained density profiles around an intruder at $z_{p, 0}=18$ in the $x z$-plane with coarse grain width of $w=0.5$ and changing density $D$. Symbols and colour bars are similar to Fig. 4.4. Here $\Delta F$ is the difference between the total contact forces on the intruder and the gravity force, where a positive number indicates an upwards lift force. The relative velocity of the intruder with the flow is indicated by $\lambda_{x}$, for more details see section 4.4 .
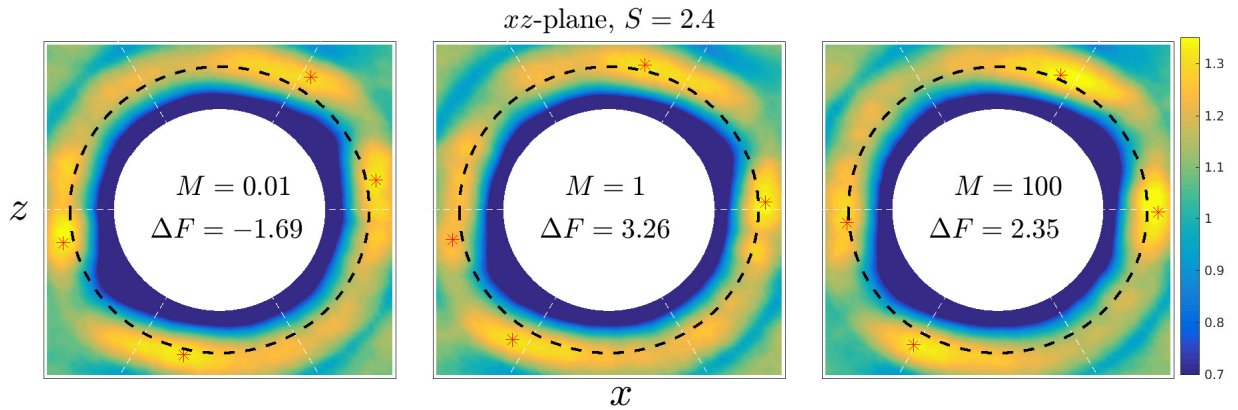

Figure 4.7: Coarse grained density profiles around an intruder at $z_{p, 0}=18$ in the $x z$-plane with coarse grain width of $w=0.5$ and changing friction ratio $M$. Here $\Delta F$ is the difference between the total contact forces on the intruder and the gravity force, where a positive number indicates an upwards lift force. Symbols and colour bars are similar to Fig. 4.4. 
equal to the average of the flow in a sphere around a singular point. For $S=0.5$ this value is already close to $\rho_{b}$. For large values of $S$ is it apparent that the density around the intruder reduces. Although a linear trend could be observed in the current region of investigation, it is highly unlikely that this continues for large $S$. Eventually for really large $S$ it should become constant, independent of $S$ for $S \rightarrow \infty$.

Variations of $\rho_{L}$ as a function of $D$ are shown in Fig. 4.8(b). For a wide range of values of $D$ there appears to be a plateau of constant $\rho_{L}$, yet for larger and smaller values of $D$ the density seem to reduce slightly. One possible theory for this could be that as $D$ increases, the particle has an increased relative velocity compared to the surrounding particles, creating a void in its wake. This effect can also be observed in Fig. 4.6.

The effect of friction on the layer density seems to be remarkably small, which supports the observation in Fig. 4.7 that the density profile around the intruder is barely affected by the friction. A small trend could be noted where low friction shows a slightly more denser layer than for a highly frictional intruder.
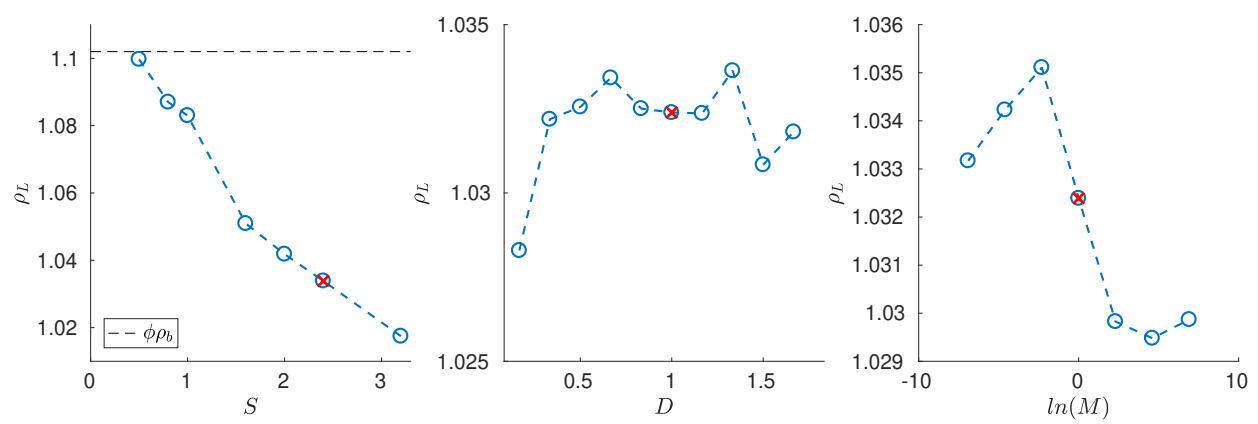

Figure 4.8: The average density of the first layer around the intruder particle for different properties. (a) $z_{p, 0}=$ 23, $D=1$ and $M=1$, (b) $z_{p, 0}=8, S=2.4$ and $M=1$, and (c) $z_{p, 0}=18, S=2.4$ and $D=1$. Note the different vertical axis. The red cross marks similar intruder properties between the graphs.

\subsubsection{COORDINATION NUMBER}

The density profiles are related to the average number of contacts of bulk particles with the intruder, the coordination number,

$$
Z=\frac{N_{c}}{N_{t}},
$$

with $N_{c}$ the total measured number of contacts and $N_{t}$ the number of time steps. The coordination number for $S$ is shown in Fig. 4.9(a) a clear upward trend is shown as $S$ increases. It is expected that for large values of $S$ the surface area is the dominant factor, $Z \propto S^{2}$. A quadratic fit with an offset seems to work really well, yet for $S<1$ a different behaviour is observed which appears to be roughly linear. This change in behaviour could be related to asymmetric segregation between large and small particles [14].

To understand this, define the number of particles inside the first layer,

$$
N_{p}=\frac{\rho_{L} V_{L}}{\rho_{p} V_{b}}=\frac{\rho_{L}}{\rho_{b}}\left(6 S^{2}+12 S+8\right),
$$


with $V_{L}$ the volume of the layer and $V_{b}$ the volume of the bulk particles. From Fig. 4.8 it was shown that although the density decreases, this is a minor effect and hence the number of particles inside the layer could be approximated with $N_{p}=\phi\left(6 S^{2}+12 S+8\right)$ for small values of $S$ as can be seen in Fig 4.10(a).

The efficiency of these particles to make a contact with the intruder is defined as $e_{c}=\mathcal{Z} / N_{p}$ and this is shown in Fig. 4.10(b). For $S=1$ it takes roughly 5.5 particles to create a contact on the intruder. As the size ratio increases this efficiency increases, implying that more particles are packed efficiently within the layer. It is this effect that could explain why the intruder $S=2.4$ in Fig. 4.2 shows extra layers, because the particles inside the layer themselves form a shell like structure. By definition the maximum value of $e_{c}$ could be one, yet the actual limit is elusive. As $S \rightarrow 0$ the efficiency declines to zero as a point will never contact any bulk particles. The fit $a_{c}\left[1-e^{\left(-S_{c}^{b}\right)}\right]$ captures $e_{c}$ pretty well, obtaining a lower limit of zero and predicting an upper limit of $e_{c}^{\infty}=0.3$.

The dramatic decline in efficiency for $S<1$ could be explained by Fig. 4.10(c) which shows the contact probability which is defined as $P_{c}=T_{c} / T_{s}$, where $T_{c}$ is the time the particle has at least one contact and $T_{s}$ is the total simulation time. A value smaller than one implies that the intruder is in free flight, not partaking in the flow. A curious observation is that even for $S=1$ the intruder is not always in contact with the other particles, roughly $8 \%$. At $S>1.6$ the particle will practically be always in contact with at least one other particle. Small particles simply do not partake in the fluid as they reside in voids generated by the larger bulk particles.

Combining the observations from $N_{p}$ and $e_{c}$ yields an estimation for the number of contacts in a granular flow

$$
\mathcal{Z}=N_{p} a_{c}\left[1-e^{\left(-S^{b_{c}}\right)}\right]
$$

This relation now also captures the low $S$ values and physically sensible limits. The dependency of $e_{c}$ on $\phi$ would be interesting future work.

The dependence of $\mathcal{Z}$ on $D$ is shown in Fig. 4.9(b). There is a visible trend that is very similar to Fig. 4.8(b) Because in this situation the size ratio is constant, the number of particles in the layer only vary with $\rho_{L}$, yet this small deviation in $\rho_{L}$ could cause a particle to lose it's contact with the intruder.

The effect of $M$ on $Z$ is shown in Fig. 4.9(c). A clear trend can be observed between no friction and high friction, captured really well with an error function. Interestingly, this might be inversely related to Fig. 4.8(c).

\subsection{VELOCITY}

An important aspect of the system shown in Fig. 4.1(b) is the shear. How this shear flow is locally affected by the intruder properties is investigated in this section. The effect on the streamlines is investigated in section 4.4.1. Additionally the translational velocity $\boldsymbol{u}_{p}$ and angular velocity $\omega_{y}$ of the intruder are compared with the reference flow. Notation is as follows. Denoting the velocity field of the bulk as $\boldsymbol{u}=(u, v, w)^{T}$, the relative velocity field around the particle can be expressed as $\boldsymbol{u}_{r e l}=\boldsymbol{u}_{p}-\boldsymbol{u}$, where $\boldsymbol{u}_{p}$ is the particle velocity. 

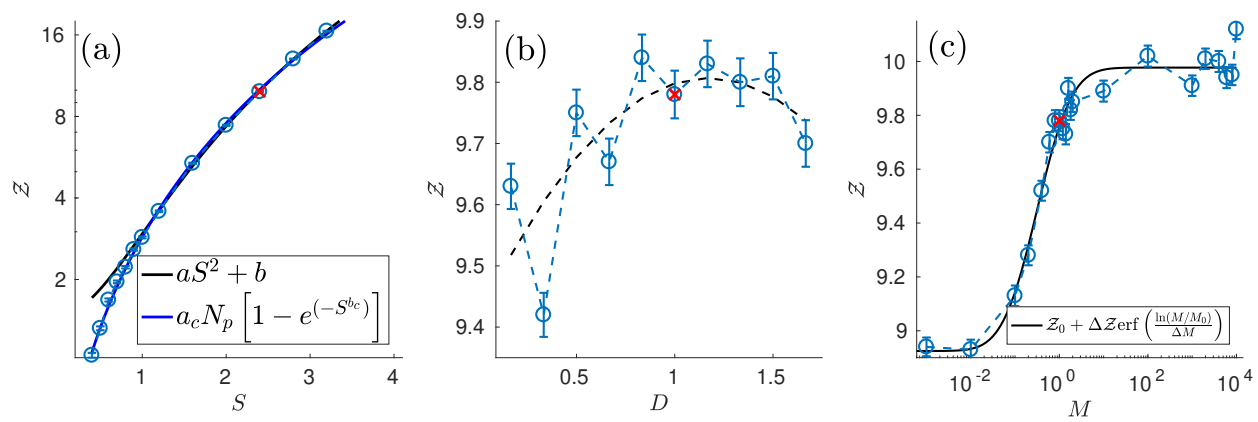

Figure 4.9: Coordination number $\mathcal{Z}$ of the intruder. (a) Coordination number as function of $S$. quadratic fit parameters are $a=1.47$ and $b=1.48$ and the second fit has values of $a_{c}=0.3121$ and $b_{c}=0.6638$. (b) Coordination number as a function of $D$, with the dashed back line as visual guide. (c) Coordination number as a function of $M$ with fit function parameters $\mathcal{Z}_{0}=9.45, \Delta \mathcal{Z}=0.53, M_{0}=0.32$ and $\Delta M=2.0$. The red cross marks similar intruder properties between the graphs.
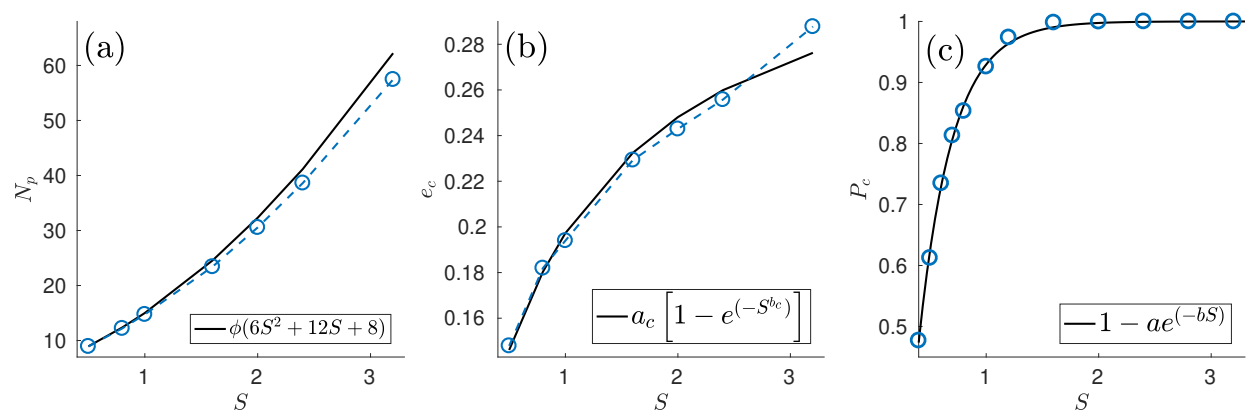

Figure 4.10: (a) Average number of particles located in the first layer around the intruder particle. (b) Contact efficiency of particles in first layer around the intruder. Fit values are $a_{c}=0.3121$ and $b_{c}=0.6638$. (c) Probability of the intruder having at least one contact with a bulk particle. Fit values are $a=2.01$ and $b=3.35$. 


\subsubsection{FLUID VELOCITY PROFILES}

The relative velocity profile around an intruder in the $x z$-plane for different $S$ is shown in Fig. 4.11. Consider the case where the intruder has the same size as the bulk particles, $S=$ 1. The flow has a positive shear rate $\dot{\gamma}$ and hence at the top of the intruder the particles move from left to right and at the bottom the particles move from right to left.

The small particles appears to reside in a layer that stretches from left to right. This layer has approximately the height of the bulk particle. Inside the layer there is some circulation, however the streamlines indicate that particles present in the layer stay approximately in the layer. The extent of this feature is larger than four times the bulk particle diameter, however on the right side of the intruder the layer starts to diminish. This velocity layer explains the high density peak left and right of the intruder in Fig. 4.4 as these particles have no big tendency to move up or downwards. Above and below the intruder the velocity profiles seem barely affected and remain straight lines.

As $S$ increases, it does not "fit" in the layer anymore and hence it has to push particles away on the top and bottom, which can clearly be observed in Fig. 4.11. The layers remain present in the profile, and their height remains approximately the height of a bulk particle. When $S \rightarrow \infty$ it is expected that the layers reduces to a single line obtaining a stagnation point at the intruder, equivalent to a stagnation point in a standard fluid. For $S=2.4$ a clear difference in layer position can be observed left and right of the intruder. The right layer appears to be slightly higher than the left layer. This effect can also be observed in the density profile, Fig. 4.4, where the high density peaks left and right of the intruder show a similar effect.

The effect of density on the streamlines is shown in Fig. 4.12. At first glance no dramatic change can be observed between various values of $D$. However, for $D>1$ it appears that the thickness of the layer on the right-hand side is reduced. As the density of the intruder increases, the intruder will start to penetrate the flow yielding a significant change in flow profile. It is believed that for $D=1.67$ this effect is slowly starting to become visible. This has connections to the slight change in structure and coordination number observed in section 4.3. Future investigations could reveal this change in flow and structure.

The effect of $M$ on the streamlines is displayed in Fig. 4.13. The difference between $M=1.0$ and $M=100$ is not profound, which could be related to the coordination number in Fig. 4.9 where the value of $M=0.5$ approximates the high friction plateau. Interestingly a huge difference between $M=1.0$ and $M=0.01$ can be observed. The effective layer seems to change structure yielding a double layer structure and reducing the effective shear zone area in the top-left and bottom right of the intruder. Additionally the effect of the intruder with respect to streamlines above and below the intruder is reduced significantly, with practically straight lines one bulk particle above the intruder. Curiously, this effect is not very pronounced in the density field around the intruder, see Fig. 4.7. A reduction in the compressional region of the flow could be the mechanism behind the reduced lift force observed in [33].

The length of the streamlines is an indication of the disturbance of the flow away from the particle. When the lines are straight, they have the domain length $L_{x}$. Fig. 4.14 shows the non-dimensional streamline length $L_{s}(z)$ for $z \in\left[0, L_{z} / 2\right]$. Only streamlines that reach the other side of the domain are taken into account to avoid the circulation 

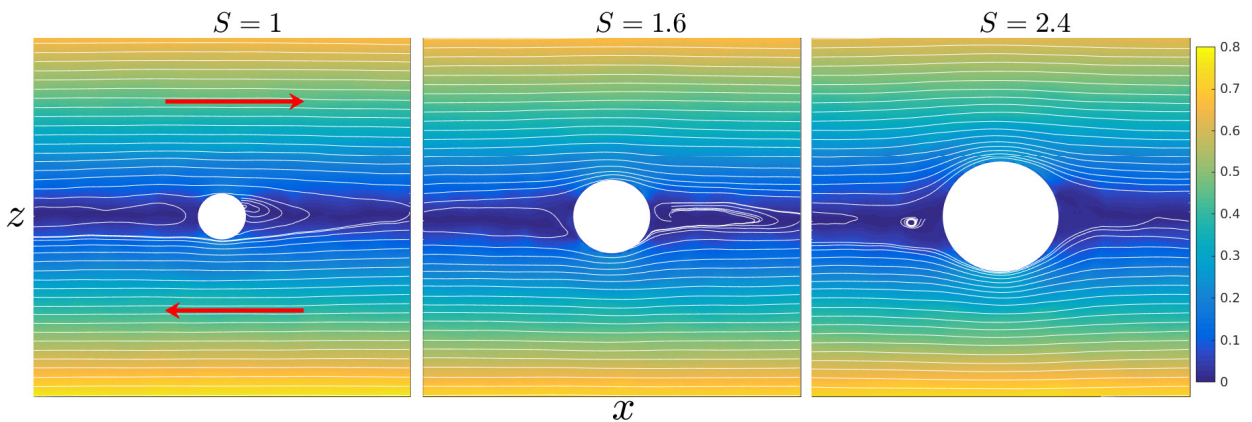

Figure 4.11: Magnitude of the relative velocity, $\left|u_{\text {rel }}\right|$ around the intruder particle for various $S$. The relative velocity at the height of the intruder is approximately zero by definition. The shear rate $\dot{\gamma}=\partial u_{x} / \partial z$ is positive. The intruder is located at approximately $z=23$.
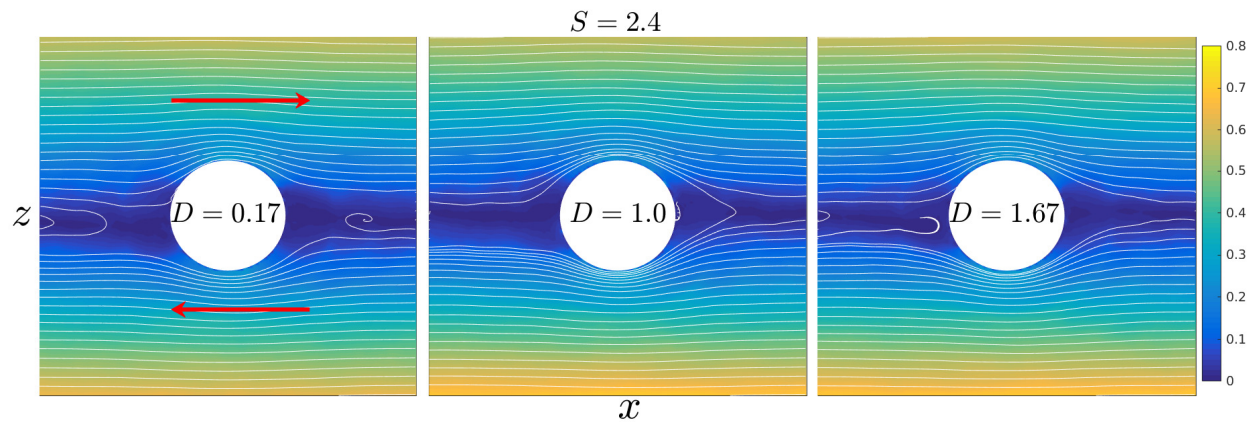

Figure 4.12: Magnitude of the relative velocity, $\left|u_{r e l}\right|$ around the intruder particle for $S=2.4$ and various $D$ at a height of approximately $z_{p, 0}=18$. The shear rate $\dot{\gamma}$ is positive. The color bar is identical to Fig. 4.11.
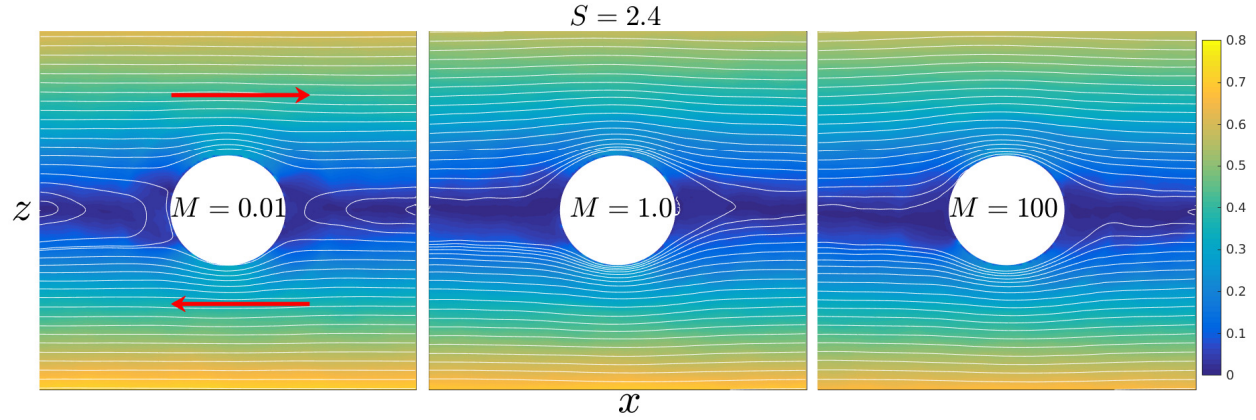

Figure 4.13: Magnitude of the relative velocity, $\left|u_{r e l}\right|$ around the intruder particle for $S=2.4$ and various $M$ at a height of approximately $z_{p, 0}=18$. The shear rate $\dot{\gamma}$ is positive. The color bar is identical to Fig. 4.11. 
areas in the flow layer.

For $S=1$ a fluid layer of roughly 0.5 can be observed. Between $z=0.5$ and $z=0.8$ a short disturbance in the streamlines can be measured and for $z>0.8$ the streamlines are practically straight. The disturbance length scale is defined at the point when $L_{s} / L_{x}=$ 0.999 . For $S=1$ This is roughly $1.6 r_{p}$ and for $S=2.4$ roughly $2.0 r_{p}$. Changing the density ratio does not show any significant differences, indicating that the flow disturbance is not influenced by the density of the particle, but only by the size. When considering the friction clear deviations can be observed again. Small friction reduces the influence range to $1.5 r_{p}$. The difference between $M=1$ and $M=100$ having both approximately an influence range of $2 r_{p}$.
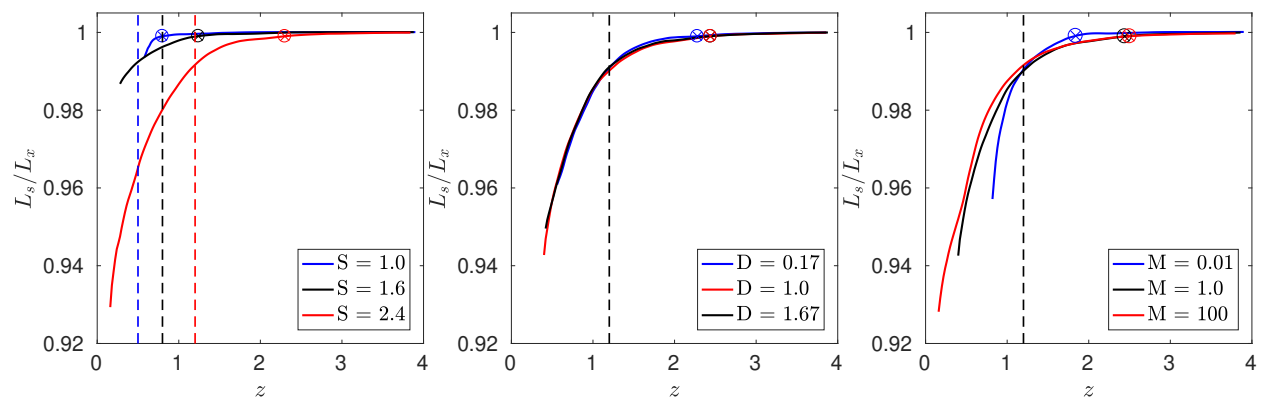

Figure 4.14: The streamline lengths divided by the domain lengths for various intruder properties. The crossed circles mark the value of $L_{S} / L_{x}=0.999$ and the dashed lines indicate the particle radius.

\subsubsection{LAG VELOCITY}

As the particle changes properties, it starts to interact differently with the surrounding particles. One of the measurements of this behaviour is the lag velocity, which recently has been linked to segregation lift force [33]. Here the lag velocity in $x$-direction is defined as

$$
\lambda_{x}=u_{p}-u^{R}
$$

where $u_{p}$ is the velocity of the particle and $u^{R}$ is the reference velocity of a flow without the intruder, at the position of the intruder. Note that definition is different compared to [33], where the flow velocity including the intruder was used.

The lag of the intruder in a chute flow with an angle of $\theta_{c}=22^{\circ}$ is shown in Fig. 4.15(a). At $S=1$ the lag velocity is expected to be zero due to symmetry, yet this is not the case. It is possible that locally the structure around the particle was different from the theoretical case. As $S>1$ a clear trend emerges of $\lambda_{x} \propto(1 / S-1)$ which is similar to the observed trend in Chapter 2, even though a different measurement of $\lambda_{x}$ is used.

Changing the density of the particle results in Fig. 4.15(b). A linear trend in $\lambda_{x}$ can be observed when varying $D$. With approximately a zero velocity at $D_{0} \approx 1.2$. The linear increase can be explained by the gravity force, which also increases linearly with $D$, which is (partially) compensated by a velocity based drag force [24]. Interestingly, the value of $D_{0}$ is in agreement with Fig. 4.9(b) where the coordination number has a peak 
at roughly the same value. For high values of $D$, the intruder starts to penetrate the flow similar to a ball falling on a bed of grains. In these situations a non-linear scaling might be observed [26].

The trend of $\lambda_{x}$ as function of $M$ is given in Fig. 4.15(c). A positive lag is observed for very small $M$ while a negative lag is observed for a large $M$. At $M \approx 1$ there seems to be great fluctuations in $\lambda_{x}$ which can't directly be explained. The overall trend follows the inverse trend of the coordination number, see Fig. 4.9(c).
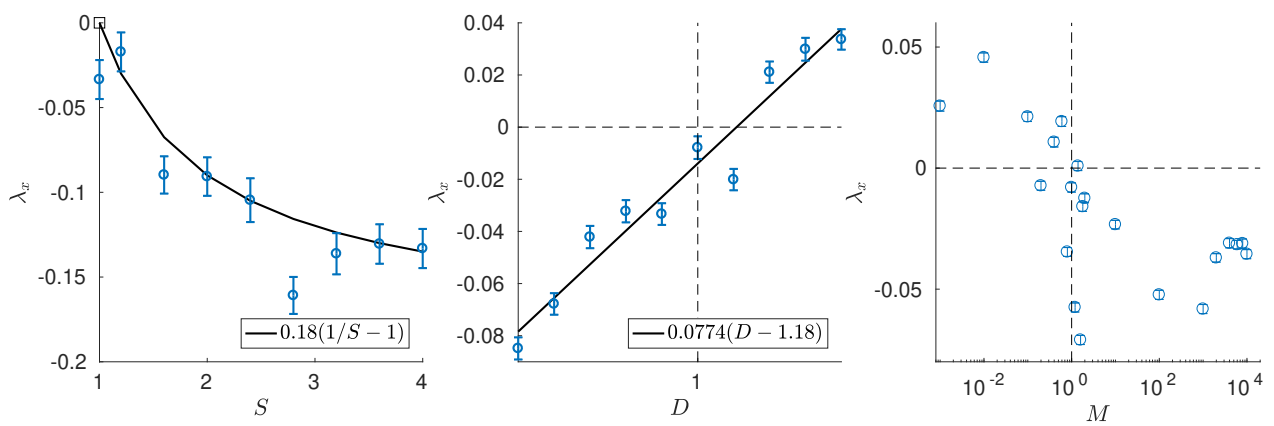

Figure 4.15: The lag velocity $\lambda_{x}$ with changing intruder parameters. (a) Lag velocity for changing $S$ at a chute angle of $\theta_{c}=27^{\circ}$ at a height of $Z_{p} \approx 23$. (b) Lag velocity for an intruder with $S=2.4$ and changing $D$ at a chute angle of $\theta_{c}=22^{\circ}$ at a height of $Z_{p} \approx 18$. (c) Lag velocity for an intruder with $S=2.4$ and changing $M$ at $\theta_{c}=22^{\circ}$ and $Z_{p} \approx 18$.

\subsubsection{ANGULAR VELOCITY}

The angular velocity of an intruder has been suggested to play an important role in segregation [34], although no direct link was given. Here the angular velocity is investigated in more detail. The angular velocity scaled by the shear rate in a chute flow of an intruder with $S=1$ at various heights is shown in Fig. 4.16. As expected due to symmetry, no rotation is observed around the $z$-and $x$-axis . A clear positive angular velocity along the $y$-axis can be observed which remains constant after scaling with the shear rate, as expected. Since the segregation force in a chute flow is height independent [32], it could be possible that $\omega_{y} / \dot{\gamma}$ is related to the segregation force.

The scaled angular velocity for different intruder properties are shown in Fig. 4.17. When the size ratio increases, the angular velocity decreases with a minimum value at $S=2$. Changing the density of the intruder practically has no influence on the angular velocity of the intruder. The angular velocity for different friction is very interesting. For large $M$ the angular velocity seems to be saturated at a constant value. A peak value occurs for small $M$, yet if the intruder particle has no friction $\left(\mu_{p b}=0\right)$, there is no incentive for the particle to rotate and no angular velocity is expected. It appears this effect will only be observed for extremely small values of $M$.

\subsection{STRESS}

In this section the effect of the intruder on the stress is investigated. The symmetric stress tensor $\boldsymbol{\sigma}$ can be decomposed in an isotropic and a deviatoric part, $\boldsymbol{\sigma}=\boldsymbol{\tau}-p \boldsymbol{I}$. Here 


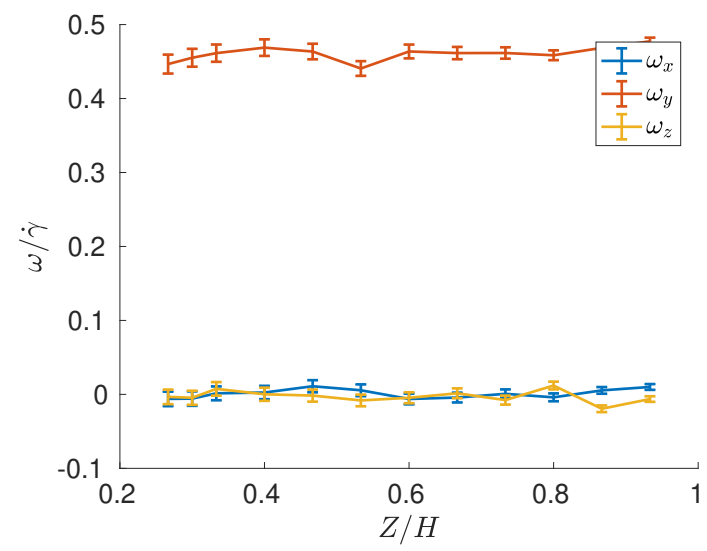

Figure 4.16: The angular velocity along the given axis $x, y$ and $z$ for an intruder particle of $S=2.4$ in a chute flow angle of $\theta_{c}=27^{\circ}$.
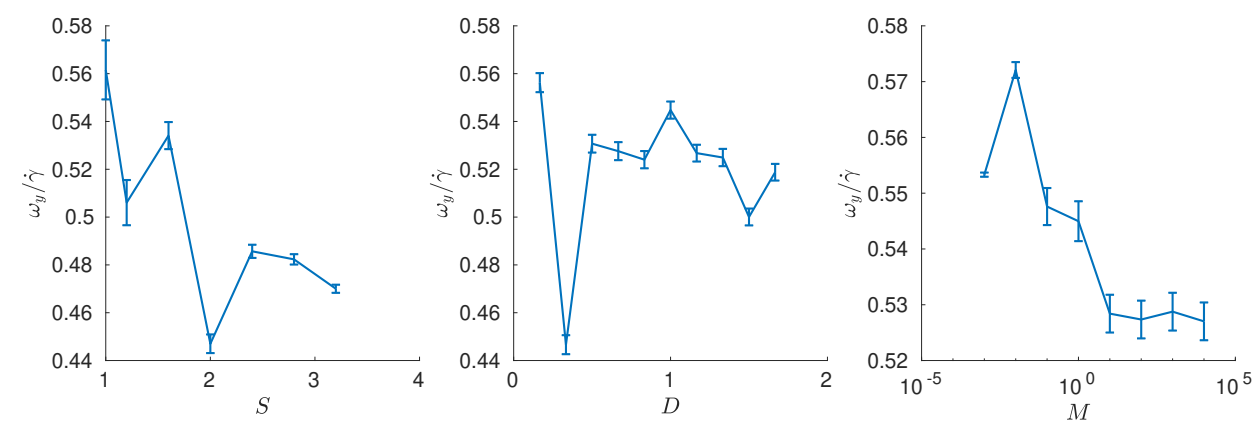

Figure 4.17: The angular velocity along the given $y$-axis for various intruder properties in a chute flow angle of $\theta_{c}=22^{\circ}$. 
$\boldsymbol{\tau}$ is the (deviatoric) shear stress tensor and $p=\operatorname{Tr}(\boldsymbol{\sigma}) / 3$ is the isotropic (compressional) pressure. The norm of the deviatoric stress tensor is given by $|\boldsymbol{\tau}|=\sqrt{\tau_{i j} \tau_{i j}}$. Section 4.5.1 discusses the pressure and section 4.5.2 discusses the shear stress. Bulk friction, the ratio between pressure and shear stress is discussed in section 4.5.3.

To investigate non-Newtonian stress effects in the flow, the deviatoric stress tensor can be transformed in its diagonal form,

$$
\boldsymbol{\tau}^{\mathrm{eig}}=\left[\begin{array}{ccc}
\tau_{1} & 0 & 0 \\
0 & \tau_{2} & 0 \\
0 & 0 & \tau_{3}
\end{array}\right]=\boldsymbol{R}^{T} \boldsymbol{\tau} \boldsymbol{R},
$$

where $\boldsymbol{R}$ is the rotation matrix containing the eigenvectors of the system and $\tau_{i}$ are the eigenvalues sorted in a descending order. The ratio of $\Lambda=\tau_{2} / \tau_{1}$ indicates the out-ofplane anisotropy within the flow and is discussed in section 4.5.4.

Another non-Newtonian effect observed in granular flows is the non-colinearity between the shear rate tensor $\dot{\gamma}$ and $\boldsymbol{\tau}$, however the shear rate tensor contains too much noise to make reasonable observations.

\subsubsection{Pressure}

The pressure field around the intruder can be decomposed in a mean hydrostatic contribution from the reference flow and a lift contribution induced by the intruder,

$$
p=p^{R}+p_{L}
$$

The hydrostatic pressure in a chute flow is given by $p^{R}=\left(1-\alpha_{z z}\right) \phi \rho_{p} g_{z}(h-z)$ where $h$ is the height of the flow and $\alpha_{z z}=1-\sigma_{z z} / p=0.05$ is a factor due to anisotropy [2]. Here we obtain the lift pressure $p_{L}$ by subtracting the pressure component far away from the intruder from the local pressure field around the intruder.

For increasing $S$ the lift pressure is shown in Fig. 4.18. For $S=1$ a layering pattern can be observed which is consistent with the density layer profile in Fig. 4.4. The pressure within the first layer does not show a significant structure. As the size ratio increases a clear the magnitude of the pressure in the compressional regions is observed. This is most likely related to the bulk particles explicitly have to move over the intruder particles, as seen from the velocity profiles, Fig. 4.11. The pressure increase seems asymmetric with more pressure at the bottom side, yielding lift forces in both the $x$ - and $z$ direction.

Interestingly, in the case of $S=2.4$ the pressure region on the bottom is divided into two parts which extend for roughly two to three layers, marked by the dashed red line. The angles are roughly $25^{\circ}$ and $60^{\circ}$. It is possible that this is caused by the structure having two preferred particle positions in the compressional region.

The lift pressure field for $S=2.4$ and changing $D$ is shown in Fig. 4.19. There seems to be no significant change in pressure which, similar to $S$, could be related to the unchanged flow around the intruder, Fig. 4.12.

Variations in $p_{L}$ due to $M$ are shown in Fig. 4.20. A remarkable difference between $M=0.01$ and $M=1$ can be observed. When the intruder has barely any friction the anisotropy in pressure seems to disappear. Both $M=1$ with $M=100$ show comparable 
pressure fields. These findings are also in line with the findings observed in the velocity profile, Fig. 4.13.
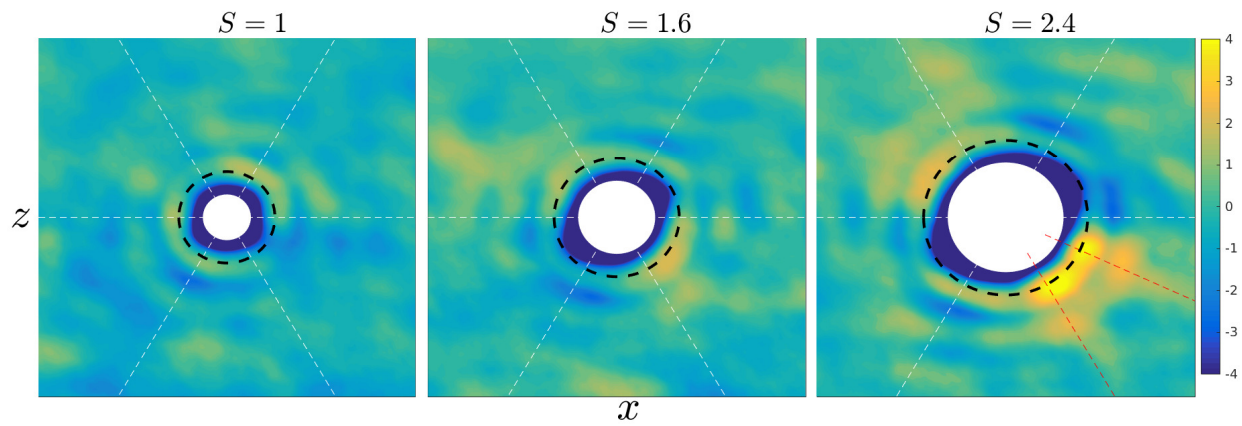

Figure 4.18: The lift pressure $p_{L}$ for different $S$ at $z_{p, 0}=23$ in the $x z$-plane with coarse grain width of $w=0.5$. The $x$ - and $z$-axis limits are $[-4,4]$. White dashed lines indicate $0^{\circ}, 60^{\circ}$ and $120^{\circ}$ angles. Red dashed lines suggest local (time-averaged) force chains.
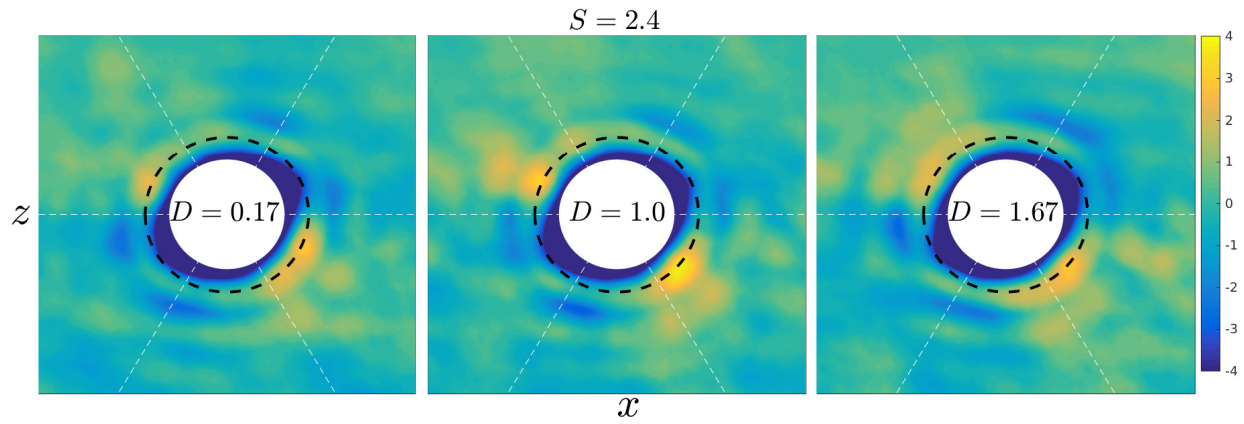

Figure 4.19: The lift pressure $p_{L}$ for $S=2.4$ and different $D$ at $z_{p, 0}=18$. Color bar and other details are similar to Fig. 4.18 .
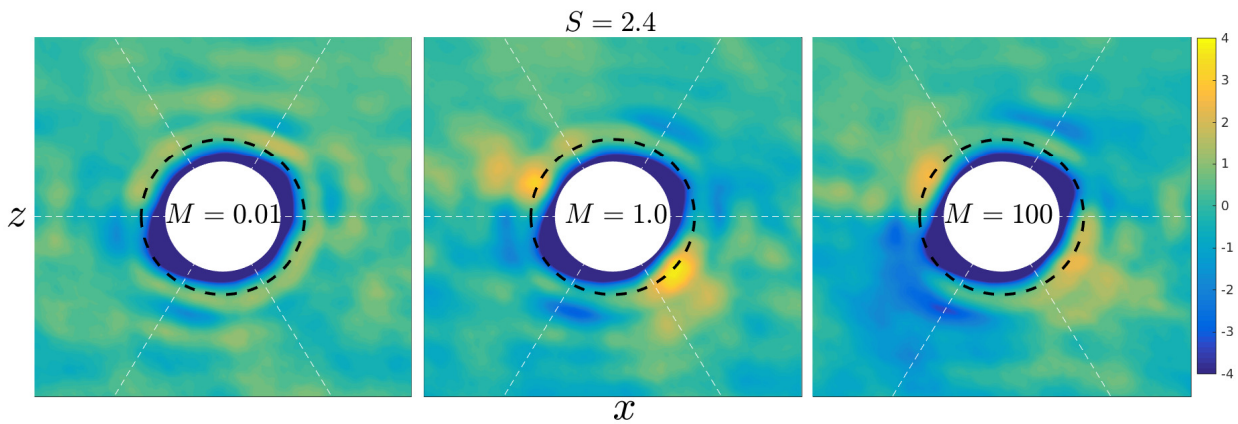

Figure 4.20: The lift pressure $p_{L}$ for $S=2.4$ and different $M$ at $z_{p, 0}=18$. Color bar and other details are similar to Fig. 4.18 . 


\subsubsection{SHEAR STRESS}

Similar to the pressure, the norm of the shear stress $|\boldsymbol{\tau}|=\sqrt{\tau_{i j} \tau_{i j}}$ contains a linear background dependency in $z, \tau^{R}$. The lift shear stress is then defined as $\tau_{L}=|\boldsymbol{\tau}|-\tau^{R}$.

The lift shear stress for $S$ is shown in Fig. 4.21. For $S=1$ there appears to be shear in the top-left and bottom-right corner close to the particle. The top-right and bottomleft show no shear. This is consistent with the compressional and tension regions. As the size ratio increases, the shear stress shows a significant increase with a peak value of roughly 4 at $S=1.6$ and roughly 12 at $S=2.4$. This increase is significantly stronger than the change in pressure. It appears that the angle of the shear stress remains $45^{\circ}$ for all $S$.

In Fig 4.22 the density of the intruder is varied and it is no surprise that the lift shear stress field doesn't show any significant change, similar to the density, velocity and pressure fields.

The friction has an enormous impact on the lift shear stress as can be observed in Fig. 4.23. When friction is almost absent, the shear stress field also is strongly diminished by a factor of roughly three. The difference between $M=1$ and $M=100$ is not very significant. Also in the case of friction the angle of shear stress remains $45^{\circ}$.

\subsubsection{BULK FRICTION}

An important quantity in continuum modeling of granular materials is the bulk friction $\mu$, defined as the ratio of shear stress and pressure,

$$
\mu=\frac{\tau}{p}
$$

Note that this is not the friction used in interaction between particles used in Table 4.1. The friction for different $S$ is shown in Fig. 4.24. For $S=1$, an almost homogeneous field of $\mu \approx 0.6$ is observed. This is higher than the theoretical bulk friction of a chute flow, $\mu=\tan \left(\theta_{c}\right)=0.40$. A mild increase of the bulk friction can be observed in the top-left and bottom-right corner at an angle of $135^{\circ}$, with a value of roughly $\mu=1.2$. As $S$ increases, this effect is enhanced significantly to a value of approximately $\mu=2.0$ for $S=2.4$. As $S$ increases, a new feature in the tensional regions can be observed, where four spots show a reduced bulk friction. The minimum bulk friction value in these spots is approximately $\mu=0.25$, which is a significant reduction compared to the average bulk friction far away from the intruder. The mechanism behind this feature is unclear, however it could be related to the detachment of the flow.

The flow profiles around different density ratios for $S=2.4$ is shown in Fig. 4.25. There does not appear to be a visible difference between different $D$, in line with observations for the shear stress and pressure in Fig. 4.22 and Fig. 4.19, respectively.

The effect of friction is shown in Fig. 4.26. For increasing $M$, the compressional region grows slightly, but the maximum measured bulk friction remains roughly $\mu=2.0$. The influence of $M$ on the reduced friction in the tensional regions is apparent when comparing $M=0.01$ and $M=1$. The region of reduced bulk friction is larger for low $M$ and the minimum value is close to $\mu=0.15$. 

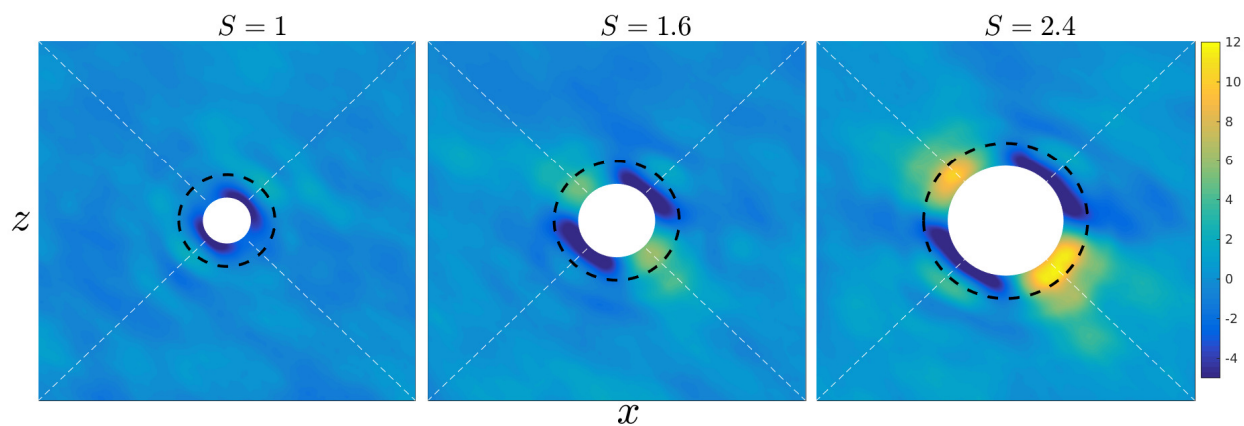

Figure 4.21: The lift shear stress $\tau_{L}$ for different $S$ at $z_{p, 0}=23$ in the $x z$-plane with coarse grain width of $w=0.5$. The $x$ - and $z$-axis limits are $[-4,4]$. White dashed lines indicate $45^{\circ}$ and $135^{\circ}$ angles.
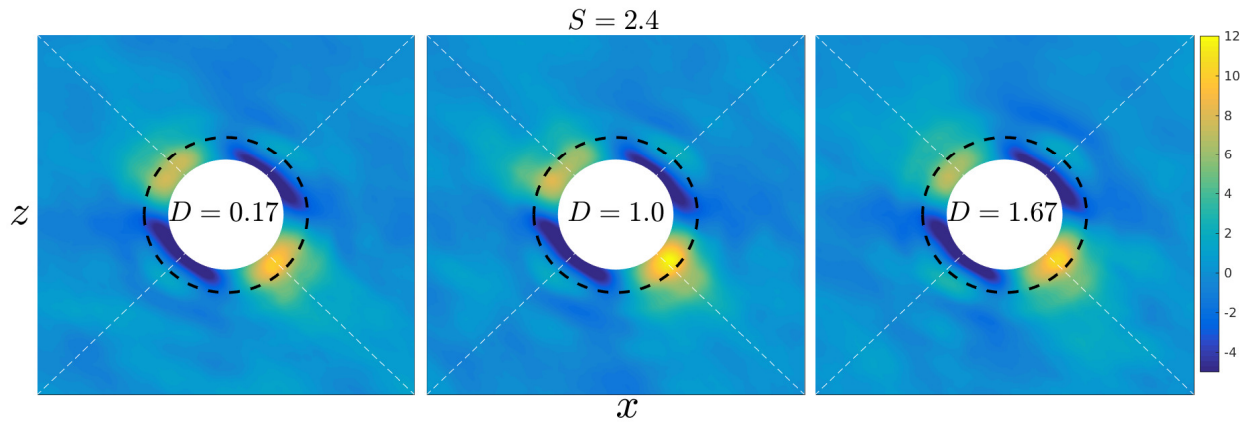

Figure 4.22: The lift shear stress $\tau_{L}$ for $S=2.4$ and different $D$ at $z_{p, 0}=18$. Color bar and other details are similar to Fig. 4.21.

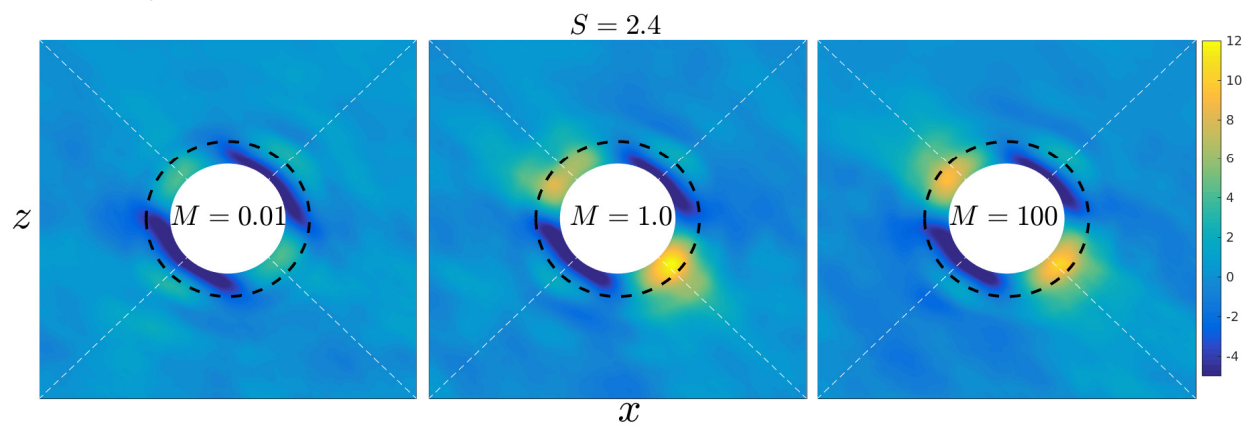

Figure 4.23: The lift pressure $p_{L}$ shear stress $\tau_{L}$ for $S=2.4$ and different $M$ at $z_{p, 0}=18$. Color bar and other details are similar to Fig. 4.21. 

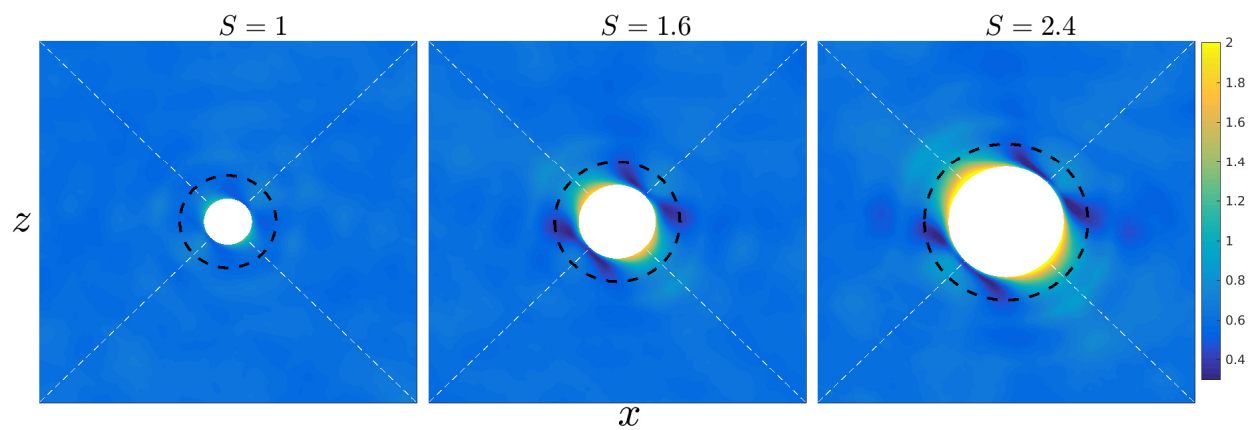

Figure 4.24: The bulk friction $\mu$ for different $S$ at $z_{p, 0}=23$ in the $x z$-plane with coarse grain width of $w=0.5$. The $x$ - and $z$-axis limits are $[-4,4]$. White dashed lines indicate $45^{\circ}$ and $135^{\circ}$ angles.
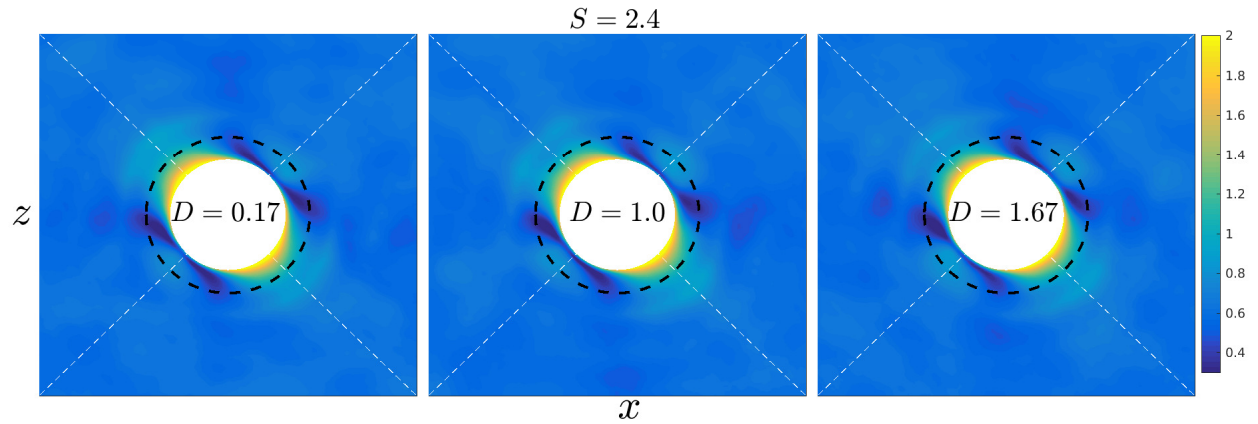

Figure 4.25: The bulk friction $\mu$ for $S=2.4$ and different $D$ at $z_{p, 0}=18$. Color bar and other details are similar to Fig. 4.24.
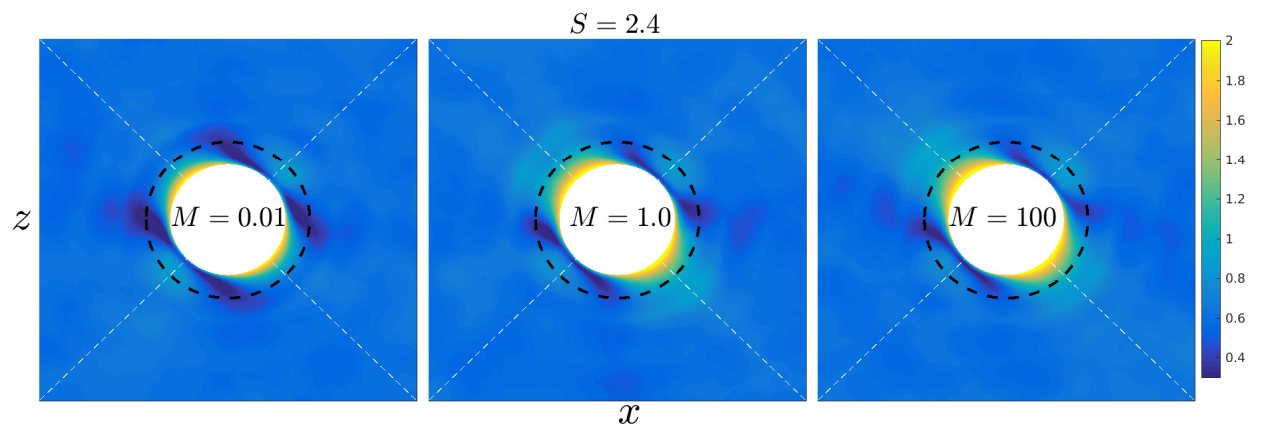

Figure 4.26: The bulk friction $\mu$ for $S=2.4$ and different $M$ at $z_{p, 0}=18$. Color bar and other details are similar to Fig. 4.24. 


\subsubsection{OUT-OF-PLANE ANISOTROPY}

The ratio of the eigenvalues $\Lambda$ is shown in Fig. 4.27. For $S=1$ a relative homogeneous field is observed with fluctuations around the value of $\Lambda \approx-0.2$ (plane stress would be $\Lambda=0$ ) , which is in agreement with observations in Ref. [2]. As the size ratio increases $\Lambda$ changes significantly. The top-left and bottom-right side of the intruder approach a value of $\Lambda=-0.5$ (dark blue) which represents an intermediate state between plane stress and axial compression $(\Lambda=-1.0)$. This is in line with the schematic picture in Fig. 4.1(b) and coincides with the change of flow profile around the intruder in Fig. 4.11. At the top-right and bottom-left corner $\Lambda$ changes sign from negative to positive and approaches a value of $\Lambda \approx 0.25$. An explanation could be that the flow gains suddenly more freedom after passing over the larger particle. Curiously both sides show two distinct peaks, but it is not directly clear what could cause this structure. Locations of positive $\Lambda$ could possibly related to the locations of reduced friction observed in Fig. 4.24. Similar to the shear stress, the angle of the compressional direct remains $45^{\circ}$ and therefore seems to be determined by the direction of shear.

Change of $\Lambda$ with respect of $D$ is shown in Fig. 4.28. Although with some fluctuations, the structure observed for $S=2.4$ does not change significantly. No significant trend can be observed which is in line with all other fields for changing $D$.

Even for different $M$, shown in Fig. 4.29 the structure remains and does not seem to be influenced much by changing the friction. It appears to be a robust feature for particles with $S>1$.

\subsection{CONCLUSIONS \& DISCUSSIONS}

In this work we have extensively analysed the continuum fields around a larger intruder particle in a granular flow. Three important intruder properties size, density and friction were varied to observe the response of the flow around the intruder.

The general structure of an intruder in a granular flow is determined by the shape of the intruder and the shear flow. The shape causes a spherical layering structure and the shear flow introduces compressional and tension regions. In the compression regions the flow is attached to the intruder and in the tension regions barely any contacts are present. The extent of the layering structure goes up to a thickness of three layers for the presently investigated intruders.

The most pronounced effect observed in the flow is caused by the size of the intruder. Bulk particles form layer structures in the flow and as the intruder increases size it does not fit in these layers. The result is a significant change in the velocity field around the intruder. This change in flow is accompanied by a change in pressure and shear stress at the compressional regions. An anisotropic structure emerges with an angle of $45^{\circ}$. The angle does not appear to depend on the intruder properties. The anisotropic stress field is asymmetric which indicates could be caused by an asymmetry in the flow. Future investigations are recommended to investigate whether a gradient in the granular flow could cause this asymmetry.

Friction has shown to be an important influence on the emerging stress fields around large intruders. When friction is almost turned off the anisotropic stress seems to almost disappear and the influence of the intruder on the velocity profile is significantly reduced. The influence of friction for $M>1$ is only minimal. 

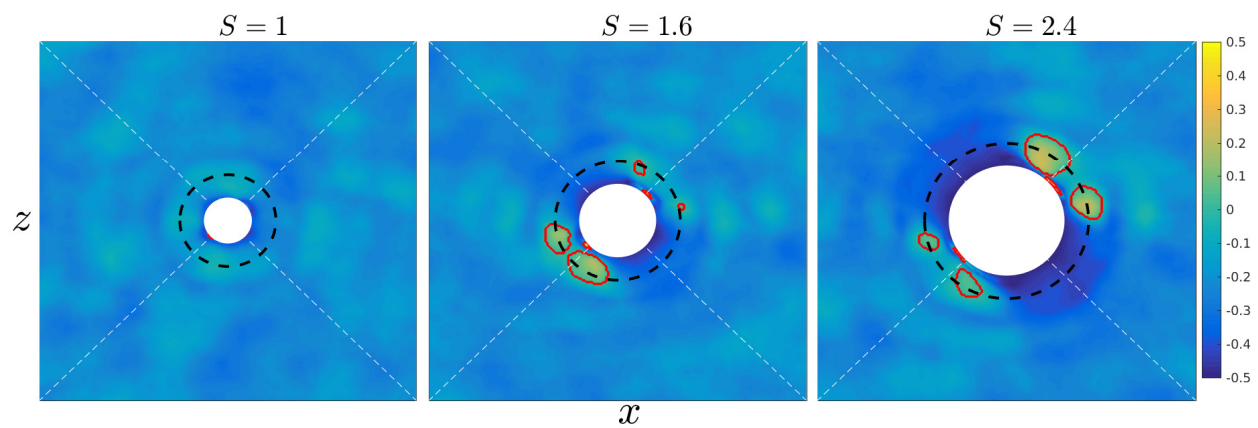

Figure 4.27: Ratio of eigenvalues $\Lambda=\tau_{2} / \tau_{1}$ shown for various values of $S$. Red lines indicate a sign switch. White dashed lines indicate $45^{\circ}$ and $135^{\circ}$ angles.
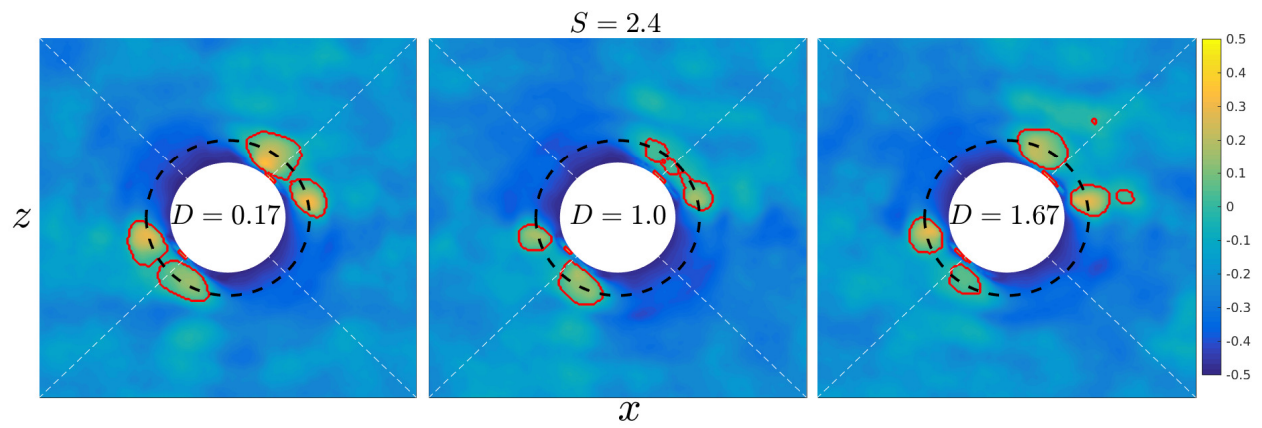

Figure 4.28: Ratio of eigenvalues $\Lambda=\tau_{2} / \tau_{1}$ shown for various values of $D$. The red lines indicate a switch in sign.
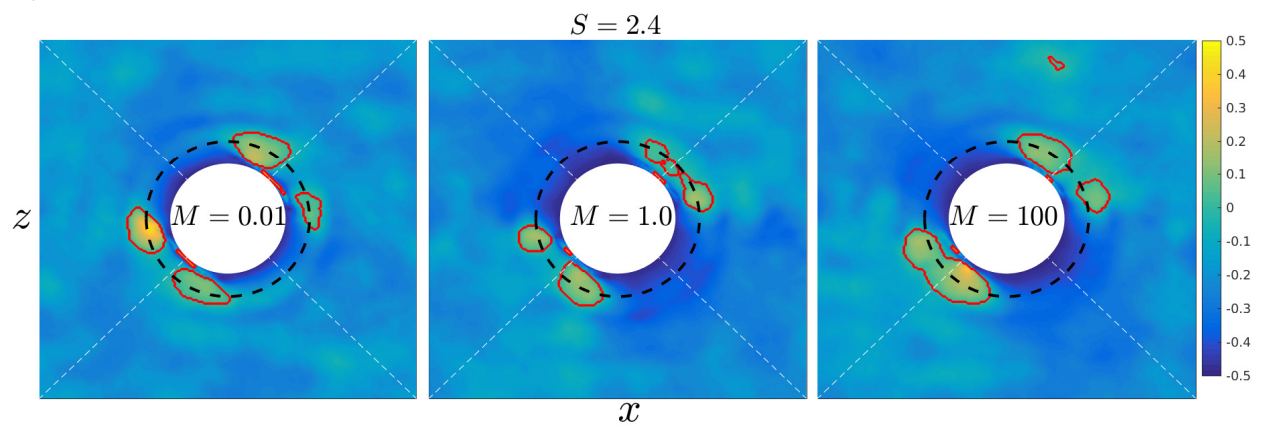

Figure 4.29: Ratio of eigenvalues $\Lambda=\tau_{2} / \tau_{1}$ shown for various values of $M$. The red lines indicate a switch in sign. 
The range of investigated densities barely have any influence on the flow. The only significant change could be measured in the lag velocity in Fig. 4.15 which is approximately linear, capturing the change of the change in gravity force. Furthermore the coordination number shows a peak roughly at the value of no lag in Fig. 4.9. This indicates that the structure is changing when increasing the density, albeit very small for the current study. Increasing the density by an extra order of magnitude would be interesting, to see the transition from a flowing particle to a penetrating particle.

The layering structure of the granular flow could have a significant effect on the drag experienced by an intruder. Specifically, in Ref. [25] a Stokesian drag law on a single (dense) intruder has been observed in a chute flow. The drag coefficient of this drag law changes for different chute flow angles and this change has not yet been understood. A possible explanation could be that if the velocity of the intruder is in the direction of the compressional regions the coefficient might be larger than if it is in the direction of the tension regions. Future investigations into this mechanism can be studied by pushing a free-flowing intruder in directions other than the gravitional direction, while keeping the chute flow angle constant.

The influence range of the intruder on the bulk flow varies, depending on the examined continuum field. When considering the density field, the maximum observed layering around the intruder increases for $S$ and for $S=2.4$ disturbances are observed three layers away from the intruder. From the limited range of $S$ investigated it is insufficient to determine how this trend develops for larger $S$ and will require further investigation. Considering the velocity profile, flow deviations up to 2 times the intruder radius are observed. The stress fields only show significant changes within the first layer of the particle.

Rich non-Newtonian effects around the intruder particle are observed for $S>1$, especially the ratio of eigenvalues and friction show significant behavioural changes. Other non-Newtonian effects such as the non-colinearity between the stress tensor and the shear rate tensor require more accurate CG fields as a numerical derivative of the velocity profile yields a high noise to signal ratio.

An important future goal is to simulate objects in granular flows. While dense granular flows have been captured with great success by the $\mu(I)$-rheology [46], it does not capture the rich layering structure observed in the density fields when following a single particle. Furthermore the flow around an object always often yields stagnation regions, regions where there is no shear present. The $\mu(I)$-rheology can't cope with this unless regularised. For sufficient large particles, the structural layering around the object is smaller than the object, such that it could be replaced by a special boundary condition. However this would require further investigation. Finally, the rich non-Newtonian effects observed are generally not included in constitutive models and could prove to be important.

\section{REFERENCES}

[1] O. Reynolds, Lvii. on the dilatancy of media composed of rigid particles in contact. with experimental illustrations, The London, Edinburgh, and Dublin Philosophical Magazine and Journal of Science 20, 469 (1885). 
[2] T. Weinhart, R. Hartkamp, A. R. Thornton, and S. Luding, Coarse-grained local and objective continuum description of three-dimensional granular flows down an inclined surface, Physics of Fluids 25, 070605 (2013).

[3] O. I. Imole, M. Wojtkowski, V. Magnanimo, and S. Luding, Micro-macro correlations and anisotropy in granular assemblies under uniaxial loading and unloading, Physical Review E 89, 042210 (2014).

[4] D. Fenistein and M. van Hecke, Kinematics: Wide shear zones in granular bulk flow, Nature 425, 256 (2003).

[5] S. Roy, S. Luding, and T. Weinhart, A general(ized) local rheology for wet granular materials, New journal of physics 19, 043014 (2017).

[6] J. M. N. T. Gray, Particle segregation in dense granular flows, Annual Review of Fluid Mechanics 50, 407 (2018).

[7] J. M. Ottino and D. V. Khakhar, Mixing and segregation of granular materials, Annual Review of Fluid Mechanics 32, 55 (2000).

[8] D. C. Hong, P. V. Quinn, and S. Luding, Reverse brazil nut problem: Competition between percolation and condensation, Physical Review Letters 86, 3423 (2001).

[9] M. J. Woodhouse, A. R. Thornton, C. G. Johnson, B. P. Kokelaar, and J. M. N. T. Gray, Segregation-induced fingering instabilities in granular free-surface flows, Journal of Fluid Mechanics 709, 543-580 (2012).

[10] I. F. Denissen, T. Weinhart, A. Te Voortwis, S. Luding, J. Gray, and A. Thornton, Bulbous head formation in bidisperse shallow granular flow over an inclined plane, Journal of Fluid Mechanics 866, 263 (2019).

[11] S. B. Savage and C. K. K. Lun, Particle size segregation in inclined chute flow of dry cohesionless granular solids, Journal of Fluid Mechanics 189, 311 (1988).

[12] T. Zhang, J. Gan, D. Pinson, and Z. Zhou, Size-induced segregation of granular materials during filling a conical hopper, Powder Technology 340, 331 (2018).

[13] A. M. Fry, P. B. Umbanhowar, J. M. Ottino, and R. M. Lueptow, Effect of pressure on segregation in granular shear flows, Physical Review E 97, 062906 (2018).

[14] K. van der Vaart, P. Gajjar, G. Epely-Chauvin, N. Andreini, J. M. N. T. Gray, and C. Ancey, Underlying asymmetry within particle size segregation, Physical Review Letters 114, 238001 (2015).

[15] Y. Fan and K. M. Hill, Phase transitions in shear-induced segregation of granular materials, Physical Review Letters 106, 218301 (2011).

[16] J. Gray and A. Thornton, A theory for particle size segregation in shallow granular free-surface flows, in Proceedings of the Royal Society of London A: Mathematical, Physical and Engineering Sciences, Vol. 461 (The Royal Society, 2005) pp. 1447-1473. 
[17] P. Gajjar and J. Gray, Asymmetric flux models for particle-size segregation in granular avalanches, Journal of Fluid Mechanics 757, 297 (2014).

[18] D. R. Tunuguntla, T. Weinhart, and A. R. Thornton, Comparing and contrasting sizebased particle segregation models, Computational Particle Mechanics 4, 387 (2017).

[19] D. R. Tunuguntla and A. R. Thornton, Balancing size and density segregation in bidisperse dense granular flows, in EPJ Web of Conferences, Vol. 140 (EDP Sciences, 2017) p. 03079.

[20] N. Thomas and U. D'ortona, Evidence of reverse and intermediate size segregation in dry granular flows down a rough incline, Physical Review E 97, 022903 (2018).

[21] N. Kumar, S. Luding, and V. Magnanimo, Macroscopic model with anisotropy based on micro-macro information, Acta Mechanica 225, 2319 (2014).

[22] V. Ogarko and S. Luding, Prediction of polydisperse hard-sphere mixture behavior using tridisperse systems, Soft Matter 9, 9530 (2013).

[23] L. Sanfratello and E. Fukushima, Experimental studies of density segregation in the $3 d$ rotating cylinder and the absence of banding, Granular Matter 11, 73 (2009).

[24] A. Tripathi and D. V. Khakhar, Density difference-driven segregation in a dense granular flow, Journal of Fluid Mechanics 717, 643-669 (2013).

[25] A. Tripathi and D. V. Khakhar, Numerical simulation of the sedimentation of a sphere in a sheared granular fluid: a granular stokes experiment, Physical Review Letters 107, 108001 (2011).

[26] S. Liu and J. J. McCarthy, Transport analogy for segregation and granular rheology, Physical Review E 96, 020901 (2017).

[27] J. Gray and C. Ancey, Particle-size and-density segregation in granular free-surface flows, Journal of Fluid Mechanics 779, 622 (2015).

[28] D. R. Tunuguntla, O. Bokhove, and A. R. Thornton, A mixture theory for size and density segregation in shallow granular free-surface flows, Journal of fluid mechanics 749, 99 (2014).

[29] Y. Srebro and D. Levine, Role of friction in compaction and segregation of granular materials, Physical Review E 68, 061301 (2003).

[30] S. Ulrich, M. Schröter, and H. L. Swinney, Influence of friction on granular segregation, Physical Review E 76, 042301 (2007).

[31] K. A. Gillemot, E. Somfai, and T. Börzsönyi, Shear-driven segregation of dry granular materials with different friction coefficients, Soft Matter 13, 415 (2017).

[32] F. Guillard, Y. Forterre, and O. Pouliquen, Scaling laws for segregation forces in dense sheared granular flows, Journal of Fluid Mechanics 807 (2016). 
[33] K. van der Vaart, M. P. van Schrojenstein Lantman, T. Weinhart, S. Luding, C. Ancey, and A. R. Thornton, Segregation of large particles in dense granular flows suggests a granular saffman effect, Physical Review Fluids 3, 074303 (2018).

[34] L. Jing, C. Y. Kwok, and Y. F. Leung, Micromechanical origin of particle size segregation, Physical Review Letters 118, 118001 (2017).

[35] D. R. Tunuguntla, A. R. Thornton, and T. Weinhart, From discrete elements to continuum fields: Extension to bidisperse systems, Computational Particle Mechanics 3, 349 (2016).

[36] I. Goldhirsch, Stress, stress asymmetry and couple stress: from discrete particles to continuous fields, Granular Matter 12, 239 (2010).

[37] L. E. Silbert, D. Ertaş, G. S. Grest, T. C. Halsey, D. Levine, and S. J. Plimpton, Granular flow down an inclined plane: Bagnold scaling and rheology, Physical Review E 64, 051302 (2001).

[38] T. Weinhart, A. R. Thornton, S. Luding, and O. Bokhove, Closure relations for shallow granular flows from particle simulations, Granular Matter 14, 531 (2012).

[39] A. R. Thornton, T. Weinhart, V. Ogarko, and S. Luding, Multi-scale methods for multi-component granular materials, Computational Methods in Matertials Science 13 (2013).

[40] T. Weinhart, D. R. Tunuguntla, M. P. van Schrojenstein-Lantman, A. J. van der Horn, I. F. C. Denissen, C. R. Windows-Yule, A. C. de Jong, and A. R. Thornton, Mercurydpm: A fast and flexible particle solver part a: Technical advances, in Proceedings of the 7th International Conference on Discrete Element Methods (Springer, 2017) pp. 1353-1360.

[41] P. A. Cundall and O. D. L. Strack, A discrete numerical model for granular assemblies, Geotechnique 29, 47 (1979).

[42] S. Luding, Cohesive, frictional powders: contact models for tension, Granular matter 10, 235 (2008).

[43] L. B. Lucy, A numerical approach to the testing of the fission hypothesis, The astronomical journal 82, 1013 (1977).

[44] V. Lebedev, Quadratures on a sphere, USSR Computational Mathematics and Mathematical Physics 16, 10 (1976).

[45] J. Baker, F. Guillard, B. Marks, and I. Einav, X-ray rheography uncovers planar granular flows despite non-planar walls, Nature Communications 9, 5119 (2018).

[46] P. Jop, Y. Forterre, and O. Pouliquen, A constitutive law for dense granular flows, Nature 441, 727 (2006). 


\section{5}

\section{FORCE MODEL FOR A LARGE INTRUDER IN DENSE GRANULAR FLOWS}

The fundamental origin of segregation of a large intruder is investigated in a monodisperse sheared granular flow. The intruder behaviour is described by a force model, which includes a segregation inducing lift force which scales proportional to $\dot{\gamma}(\partial \eta / \partial z)$. This scaling allows for a unified segregation mechanism valid for many different systems: large particles tend to move to low viscosity regions. This observation is critically important for the understanding of segregation. Moreover, it could significantly help improve continuum segregation models. The derived force model can be used to predict segregation in dense mixtures and could even capture reverse segregation.

\subsection{INTRODUCTION}

A plethora of theories have been proposed to explain size segregation in dense granular flows such as percolation, kinetic sieving, squeeze explusion and granular temperature [2-7].

Based on these theories segregation models have been developed for bidisperse mixtures of large and small particles. A gravity-driven binary mixture theory has been developed by formulating a model for kinetic sieving [8]. This was done by partitioning the pressure based on the local solids fraction of large and small particles. An improvement of this model has been made by the observation that this pressure partitioning is asymmetric $[9,10]$. Further extensions have been made by incorporating the density ratio [11]. By introducing a grain size coordinate a mixture theory for polydisperse flows was developed [12]. Alternative models consider kinetic temperature instead of gravity as the driving force behind segregation [13, 14].

The simulations in this chapter have been performed by F. Guillard [1]. 
A recent comparison of these models shows that it is not clear why the models capture 50/50 bidisperse segregation correctly [15]. Moreover, in dilute regimes (e.g. 10/90 mixtures) most models do not capture segregation as accurately [16]. Therefore, a more fundamental approach to segregation is required.

The origin of segregation is investigated by considering one large particle (intruder) in a monodisperse bulk and to capture its behaviour in a force model. A previous study with a similar aim shows that the total contact force on an intruder can be expressed by a combination of gradients in pressure and shear stress and bulk friction-dependent empirical functions [1]. Here we take a different approach by decomposing the total contact force into buoyancy, drag and lift forces, similar to a particle in a Newtonian fluid [17]. The buoyancy force has been studied in detail in Chapter 3 revealing a size ratio-dependent mechanism related to the contact density on the surface of the intruder. Drag forces on intruder particles have recently had much attention [18, 19], showing Stokes drag-like behaviour. The lift forces in such a model have not yet been investigated. Especially the recently discovered velocity lag inducing lift force in the flow-direction, observed in Chapter 2, has not yet been understood. The objective of this chapter is to capture the scaling laws of the lift forces and to complete the force model on the intruder.

To determine the lift force scalings the segregation direction is considered first. In Chapter 4 the origin of lift of an intruder has been visualised by analysing the impact of the intruder on the flow. A clear anisotropic stress profile emerges as the intruder particle does not fit in a flow layer of bulk particles. This anisotropy is likely caused by an asymmetry in the properties of the flow (i.e. shear-rate, viscosity, granular temperature). Therefore, a systematic investigation of all important flow properties is performed to determine the correct segregation direction.

The magnitude of segregation is then investigated by isolating the lift force on the intruder. In most systems this is non-trivial, due to the presence of stress gradients in the flow. However, it is possible in a plate driven sheared setup with gravity in either vertical or horizontal direction to either remove the pressure or shear stress gradient [1]. The scaling of the lift force in this configuration is then investigated in relation to the flow gradient that determines the segregation direction. This result is then combined with the buoyancy and drag forces into a force model of the intruder.

The Chapter is organised as follows. In Section 5.2 the method and simulations are discussed. The origin of segregation is investigated in Section 5.3. Combining all results lead to a force model in Section 5.4. The result is concluded and discussed in Section 5.5.

\subsection{METHODS AND SIMULATIONS}

The 2D configuration considered in this work is shown schematically in Fig. 5.1(a) and is the same as in Ref. [1]. The setup consists of a fixed rough bottom, while the top consists of a moving rough rigid plate. The plate applies a pressure $P_{0}$ on the granular material and and moves with a velocity $V$, generating a sheared flow. In the $x$-direction periodic boundaries are applied and gravity is applied under an arbitrary angle. The simulations are performed in the discrete element software LIGGGHTS [20].

The bulk material consists of disk-shaped particles with a mean diameter of $d=$ $1.5 \mathrm{~mm}$ and a density of $\rho_{b}=2500 \mathrm{~kg} / \mathrm{m}^{-2}$. A slight polydispersity of $20 \%$ is added to avoid crystaline structures. The normal contact forces between particles are modeled as 
spring-dashpot system with a stiffness of $k_{n}=81 \times 10^{4} \mathrm{~N} / \mathrm{m}$ and a dissipation coefficient of $\gamma_{n}=12 \mathrm{~kg} / \mathrm{s}$. The tangential contact forces between particles are also modeled by a spring-dashpot system with an added frictional slider. The stiffness and dissipation are $k_{t}=86 \times 10^{4} \mathrm{~N} / \mathrm{m}$ and $\gamma_{t}=\gamma_{n}$, while the sliding friction is $\mu_{b}=0.5$.

Segregation is investigated by adding a single intruder with diameter $d_{c}$ to the system at a height of $z_{p, 0}$. The intruder is attached to a vertical spring with stiffness $k_{s}=70 \mathrm{~N} / \mathrm{m}$ to allow for some vertical movement, yet keeping the intruder roughly at the same flow height. The spring force is defined as $F_{s}=-k_{s}\left(z_{p}-z_{p, 0}\right)$, where $z_{p}$ is the average position of the particle. For more details the reader is referred to Ref. [1].

Measuring the lift force on the intruder is done by investigating two specific setups. Both are shown in Fig. 5.1(b) and Fig. 5.1(c) with, respectively, vertical and horizontal gravitation. In the first system no gravity force is present in the $x$-direction, such that the lag inducing lift force, see Chapter 2, is isolated and in the second case the lift force in vertical direction can be measured directly through the spring force.
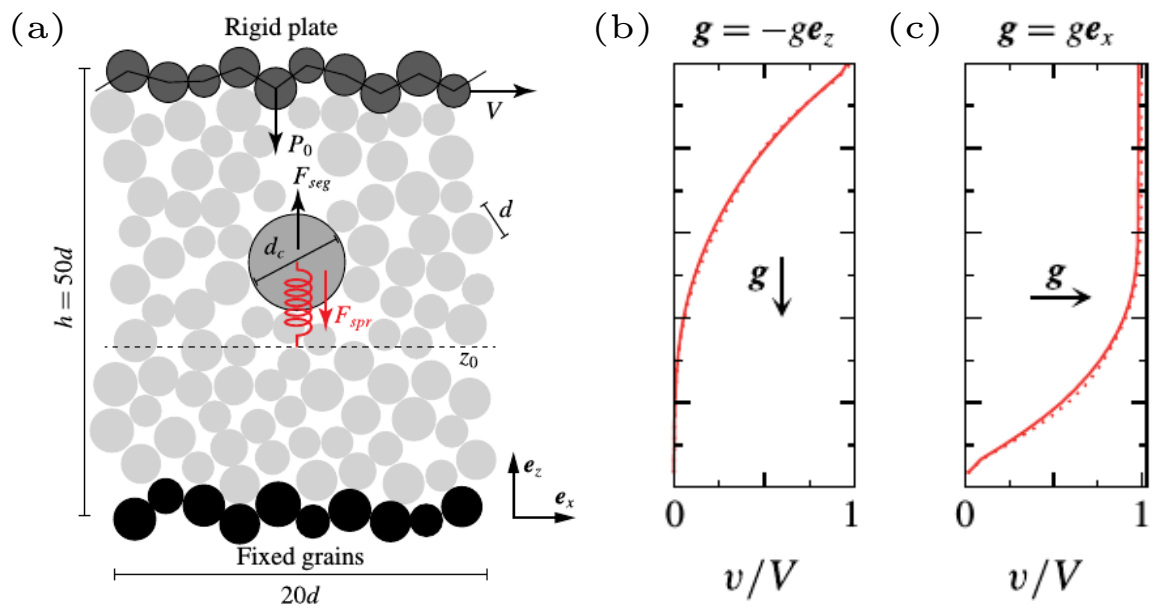

Figure 5.1: (a) Schematic of the simulation setup, with a fixed bottom and a moving plate on top. The intruder particle is attached to a spring to keep it from moving. (b) Velocity profile for a system with vertical gravity. (c) Velocity profile for a system with horizontal gravity. Schematic and graphs reproduced from Ref. [1] with permission.

\subsection{RESULTS}

\section{A. Horizontal Gravity}

Consider a sheared flow with horizontal gravity. The spring force of the intruder is shown in Fig. 5.2(a). For an intruder with $S=1$, the spring force is very small, fluctuating around zero. The gravity force is absent in the vertical direction, indicating that all the individual particle contact forces are approximately balanced. As the intruder increases in size, a 
clear positive spring force can be measured. This implies that the particle has the tendency to sink due to a downwards directed lift force.

Observations of a velocity lag were made in Chapter 2 for increasing intruder size. Here the relative velocity of the intruder with the bulk velocity is defined similarly,

$$
\boldsymbol{\lambda}=\boldsymbol{u}_{p}-\boldsymbol{u}^{R}\left(z_{p}\right)
$$

where $\boldsymbol{u}_{p}$ is the intruder velocity and $\boldsymbol{u}^{R}\left(z_{p}\right)$ is the velocity of a reference flow at intruder position $z_{p}$ without intruder present. For increasing $S$ the considered system shows a velocity rise, as can be observed in Fig. 5.2(b), which indicates that the intruder is moving faster than the flow. This could be purely caused by the gravity force, however it could also indicate a similar lift force present in the $x$-direction.
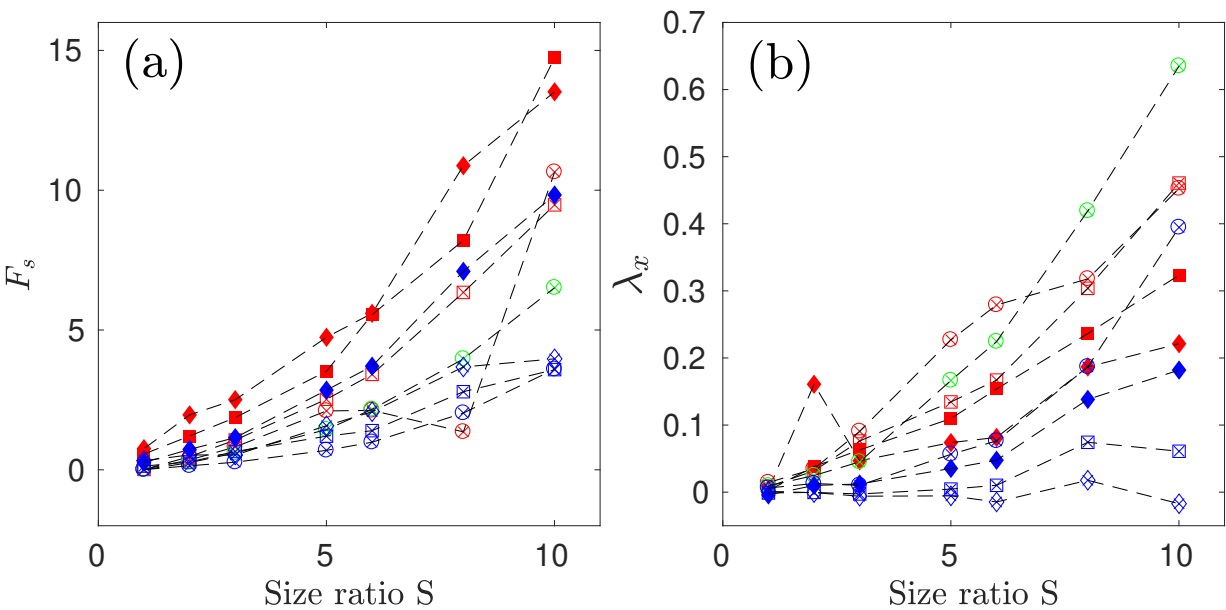

Figure 5.2: Sheared flow with horizontal gravity (constant pressure) and various boundary conditions. Plate velocity $V=\{2.5,5\} \mathrm{m} / \mathrm{s}$, respectively solid and crossed symbols. Plate pressure $P_{0}=\{1,2.5,5\}$ Pa, respectively square, diamond and triangle. Gravitational acceleration $g=\{3,5,7\} \mathrm{m} / \mathrm{s}^{2}$, respectively blue, green and red. (a) Measured spring force and (b) relative velocity of the intruder for increasing $S$. The data has been obtained from Ref. [1].

\section{B. VERTICAL GRAVITY}

Now consider a sheared flow with vertical gravity. The spring force of the system is shown in Fig. 5.3(a). At $S=1$ there is no spring force active as expected. For increasing $S$ a negative spring force emerges, implying the intruder wants to rise. In this system the gravity force is absent in the $x$-direction and therefore an imbalance in contact forces would yield a velocity lag. The relative velocity of the intruder is shown in Fig. 5.3(b), showing no velocity lag for $S=1$. However, for increasing $S$, a velocity rise emerges, implying the presence of a lift force in the $x$-direction. Note that most simulations follow a downwards curve, but for roughly three simulations alternative behaviour is observed for which no explanation has been found. 

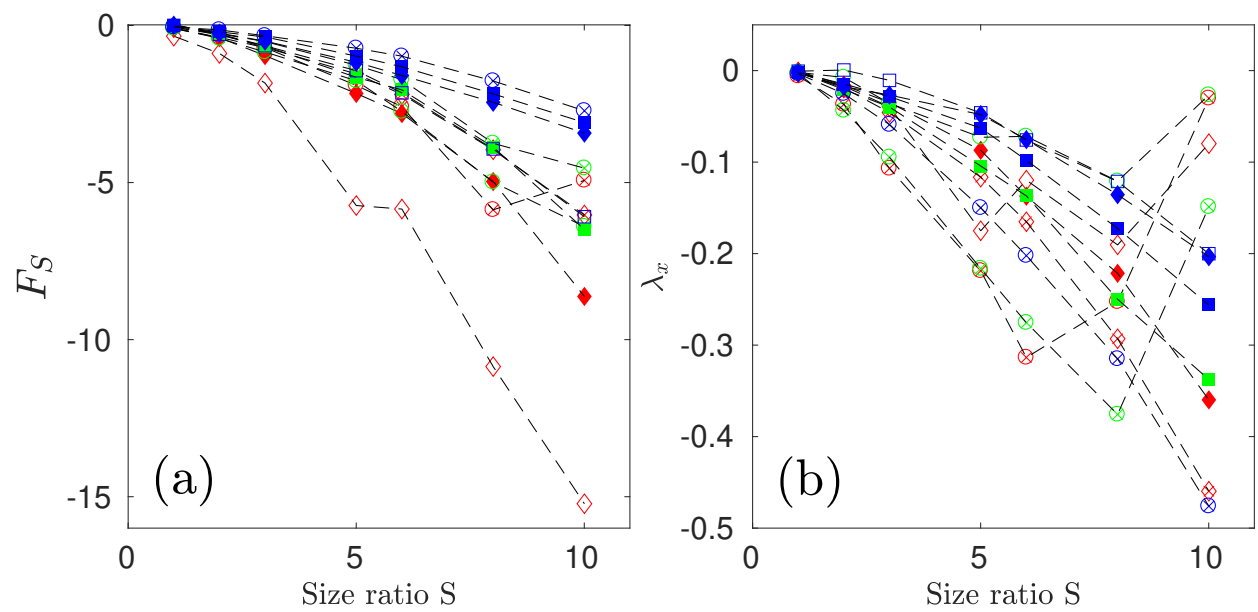

Figure 5.3: Sheared flow with vertical gravity and various boundary conditions. Shear velocity $V=$ $\{1,2.5,5\} \mathrm{m} / \mathrm{s}$, respectively open, closed and crossed symbols. Plate pressure $P_{0}=\{1,2.5,5\} \mathrm{Pa}$, respectively circle, square and diamond. Gravitational acceleration $g=\{3,5,7\} \mathrm{m} / \mathrm{s}^{2}$, respectively blue, green and red. (a) Measured spring force and (b) relative velocity of the intruder for increasing $S$. The data has been obtained from Ref. [1].

\subsubsection{SEgREgATION DIRECTION}

In the two sheared flow configurations considered thus far, the flow changes in such a way that segregation direction reverses, including the direction of the lag velocity. It is important - as a starting point - to understand in which direction the intruder segregates. To this purpose, other configurations are also considered. In Fig. 5.4(a) and Fig. 5.4(b) the sheared flows with horizontal and vertical gravity are schematically shown, together with the observed lift force directions. The other configurations shown are: a chute flow [21] in Fig. 5.4(c), a shear box [10] in Fig. 5.4(d) and a vertical chute [22] in Fig. 5.4(e). Not all systems have been investigated with a single intruder, and therefore a reasonable assumption is to take the lift force direction similar to the direction of a 50/50 mixture.

Previous investigations have linked the lift force direction with gradients in pressure $\frac{\partial p}{\partial z}$, shear stress $\frac{\partial|\boldsymbol{|}|}{\partial z}$ or granular temperature $\frac{\partial T}{\partial z}[1,22]$. Two gradients that have not yet been directly investigated to our knowledge are the shear rate gradient $\frac{\partial \dot{\gamma}_{x z}}{\partial z}$ and the gradient in viscosity $\frac{\partial \eta}{\partial z}$. These gradients are related to the shear stress, $\boldsymbol{\tau}=\eta \dot{\boldsymbol{\gamma}}_{x z}$. When only flows with a shear component of $\dot{\gamma}_{x z}$ are considered, the derivative of the shear stress becomes

$$
\frac{\partial \tau_{x z}}{\partial z}=\eta \frac{\partial \dot{\gamma}_{x z}}{\partial z}+\frac{\partial \eta}{\partial z} \dot{\gamma}_{x z}
$$

To analysis of the sign of the gradient it is convenient to introduce a non-dimensional ratio between the two terms,

$$
\alpha=\frac{\eta \frac{\partial \dot{\gamma}_{x z}}{\partial z}}{\dot{\gamma}_{x z} \frac{\partial \eta}{\partial z}} .
$$


In Fig. 5.4 the value of $\alpha$ is shown for different flow configurations, together with the sign of all gradients present in the flow. The derivation of $\alpha$ for the different configurations can be found in Appendix B, where it is assumed that the bulk friction $\mu$ follows the wellknown $\mu(I)$-rheology [23, 24]. The sign of $\dot{\gamma}_{x z}$ and $\frac{\partial \dot{\gamma}_{x z}}{\partial z}$ can be determined directly from the velocity profiles. The direction of the granular temperature can be estimated through the observation of $T \propto \dot{\gamma}^{2}$ [25], yielding $\frac{\partial T}{\partial z} \propto \dot{\gamma}_{x z} \frac{\partial \dot{\gamma}_{x z}}{\partial z}$. The sign of the viscosity gradient can be obtained with

$$
\operatorname{sgn}\left(\frac{\partial \eta}{\partial z}\right)=\operatorname{sgn}\left(\frac{1}{\alpha \dot{\gamma}_{x z}} \eta \frac{\partial \dot{\gamma}_{x z}}{\partial z}\right),
$$

where the viscosity is by definition always positive. This definition is ill-defined in case of a shear box where $\alpha=0$. Instead, the viscosity gradient can be expressed as

$$
\frac{\partial \eta}{\partial z}=\frac{1}{\left|\dot{\gamma}_{x z}\right|} \beta(I) \frac{\partial p}{\partial z}
$$

where $\beta(I)$ is a positive valued function, see Appendix B for details.

Next, we investigate if a single gradient can capture segregation in the $z$-direction correctly. The gradient $\frac{\partial \dot{\gamma}_{x z}}{\partial z}$ can be eliminated when considering the shear box, see Fig. 5.4(d). Here positive segregation is present while $\frac{\partial \dot{\gamma}_{x z}}{\partial z}$ is absent. The gradient in granular temperature shows an incompatibility when considering the sheared flow with $g_{x}=0$ in Fig. 5.4(b) and the chute flow in Fig. 5.4(c). In both cases an upward lift force is observed, while the gradient in temperature changes sign. Note that this is in contrast with previous theories considering granular temperature [22]. The pressure gradient is absent in the sheared flow with horizontal gravity and the vertical chute while segregation is still present, see Fig. 5.4(a) and Fig. 5.4(e). Therefore, the pressure gradient is also eliminated. The only remaining gradient that captures all lift force directions correctly is $\frac{\partial \eta}{\partial z}$. Note that this does not imply that other gradients do not cause lift forces on the intruder. Instead, $\frac{\partial \eta}{\partial z}$ could be identified as the dominating gradient causing the asymmetry observed in Chapter 4.

A lift force in the flow direction has only recently been observed [21] and therefore it is not known for all configurations. Interestingly, the direction of lift force in the $x$ direction for $\dot{\gamma}_{x z}>0$ is also captured by $\frac{\partial \eta}{\partial z}$. By means of a mirror argument, the segregation in $x$-direction should change sign when $\dot{\gamma}_{x z}<0$. In Fig. 5.5 all possible segregation directions are shown, based on the sign of both $\frac{\partial \eta}{\partial z}$ and $\dot{\gamma}_{x z}$.

\subsubsection{SEGREGATION MECHANISM}

The next step is to determine the mechanism for segregation. Since the direction of the lift force $F_{L}$ can be described by the sign of $\dot{\gamma}_{x z}$ and $\frac{\partial \eta}{\partial z}$, an obvious choice is to test $F_{L} \propto \dot{\gamma}_{x z} \frac{\partial \eta}{\partial z} V_{p}$, where $V_{p}$ is the volume of the intruder. For most systems it is non-trivial to isolate the lift force, especially due to gravity generating buoyancy-like forces, see Chapter 3. However, the sheared systems in Fig. 5.4(a) and Fig. 5.4(b) give an unique opportunity due to an absence of gravity force in the $z$ - and $x$-direction, respectively.

Firstly, consider a sheared flow with horizontal gravity, see Fig. 5.4(b). In the absence of a gravity force in the $z$-direction, the spring force purely compensates an emerging lift 
(a) Sheared flow, $g_{z}=0$

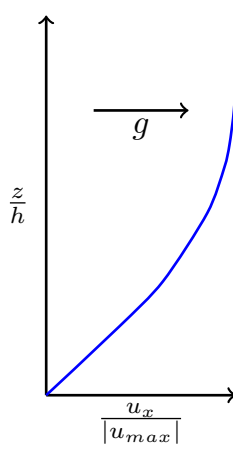

(c) Bagnold flow

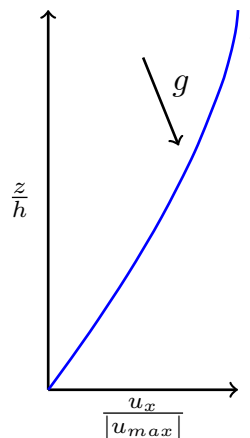

(b) Sheared flow, $g_{x}=0$

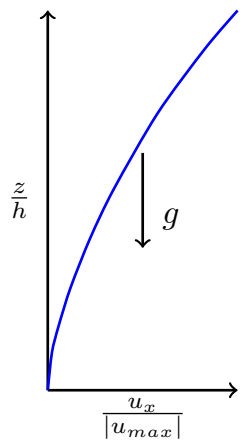

$$
\begin{array}{ll}
\dot{\gamma}>0 & \frac{\partial \dot{\gamma}}{\partial z}>0 \\
\alpha=-1 & \frac{\partial T}{\partial z}>0 \\
\eta>0 & \frac{\partial \eta}{\partial z}<0 \\
\frac{\partial p}{\partial z}<0 & \frac{\partial|\tau|}{\partial z}=0 \\
r \underset{x}{\stackrel{\uparrow}{\longrightarrow}}
\end{array}
$$

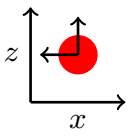

(d) Shear box, $\dot{\gamma}>0$

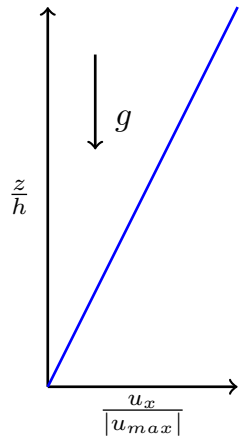

(e) Vertical chute

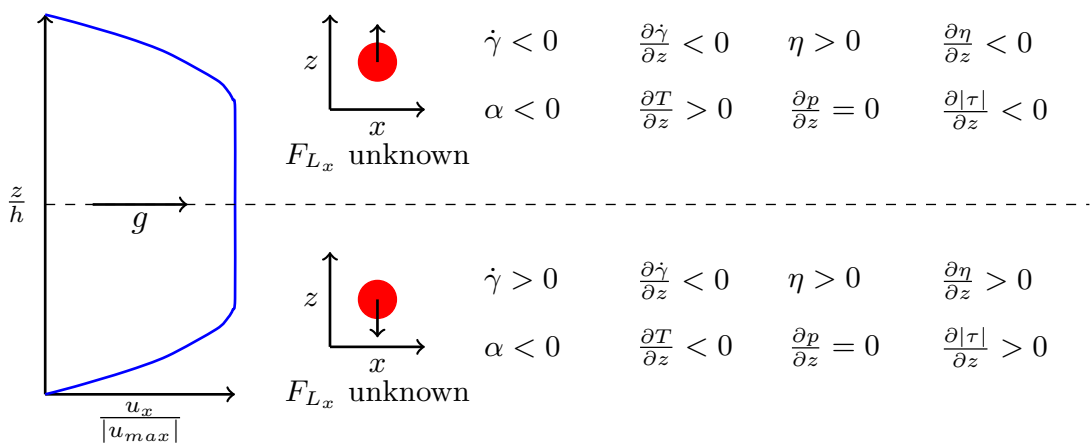

Figure 5.4: Different configurations showing the approximated non-dimensionalised velocity profiles $u(z)$, segregation direction of the (red) intruder and the sign of flow gradients. The gradients have been obtained as discussed in section 5.3.1. 


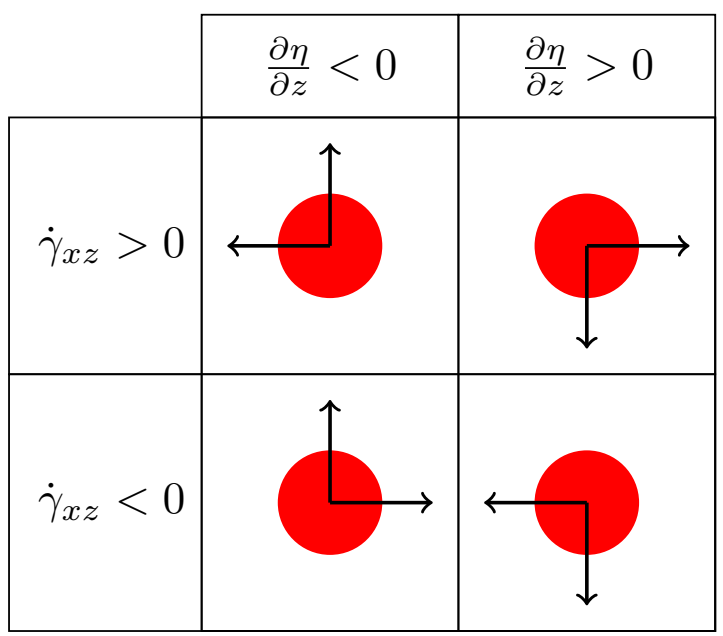

Figure 5.5: Segregation directions quantified by the signs of $\dot{\gamma}_{x z}$ and $\frac{\partial \eta}{\partial z}$.

force. Since the intruder is in a time-averaged steady state, the force balance in the $z$ direction is given by $F_{L_{z}}+F_{s}=0$. The scaled lift force in Fig. 5.6(a) shows a clear collapse for most simulations. For small values of $S$ some simulations go to zero as expected, while some simulations do not. A possible cause for this could be high fluctuations observed for intruders with $S=1$. For large values of $S$ the value seems to converge to a value of approximately one.

Secondly, consider the $x$-direction of a sheared system with gravity in the vertical direction, see Fig. 5.4(a). The presence of a lag velocity suggests a balance between two forces. One forces is related to $\lambda_{x}$, commonly referred to as a drag force. The other force is the lift force, $F_{L_{x}}$. The force balance can therefore be written as $F_{L_{x}}-F_{d}\left(\lambda_{x}\right)=0$. The magnitude of either the lift nor the drag force can be measured, due to a lack of spring force in the $x$-direction. However, it is possible to test whether $F_{L_{x}} \propto \dot{\gamma}_{x z} \frac{\partial \eta}{\partial z}$. In a 3D system a Stokesian drag force has been observed with the scaling $F_{d} \propto \eta \lambda_{x} d_{p}$ [26]. Therefore, a plausible scaling for a 2D system is $F_{d} \propto \eta \lambda_{x}$. Assuming that $F_{L_{x}} \propto \dot{\gamma}_{x z} \frac{\partial \eta}{\partial z} V_{p}$, a collapse should be observed when $\eta \lambda_{x}$ is correctly rescaled. In Fig. 5.6(b) the lag is rescaled accordingly and shows a good collapse for most simulations.

Both systems show a lift force that indeed does scale with $\dot{\gamma} \frac{\partial \eta}{\partial z}$, capturing both segregation in $x$ - and $z$-direction with a unified mechanism. The segregation mechanism can be understood when considering the velocity profiles observed in Chapter 4 . Here a large intruder does not fit inside a layer of bulk particles. An increased effort of the bulk particles is required to move around the intruder, yielding anisotropic pressure and shear stress fields. Bulk particles in low viscosity regions move more easily around the intruder than bulk particles in high viscosity regions. Therefore, the the bulk particles in the more viscous areas push the intruder towards low viscosity regions. 

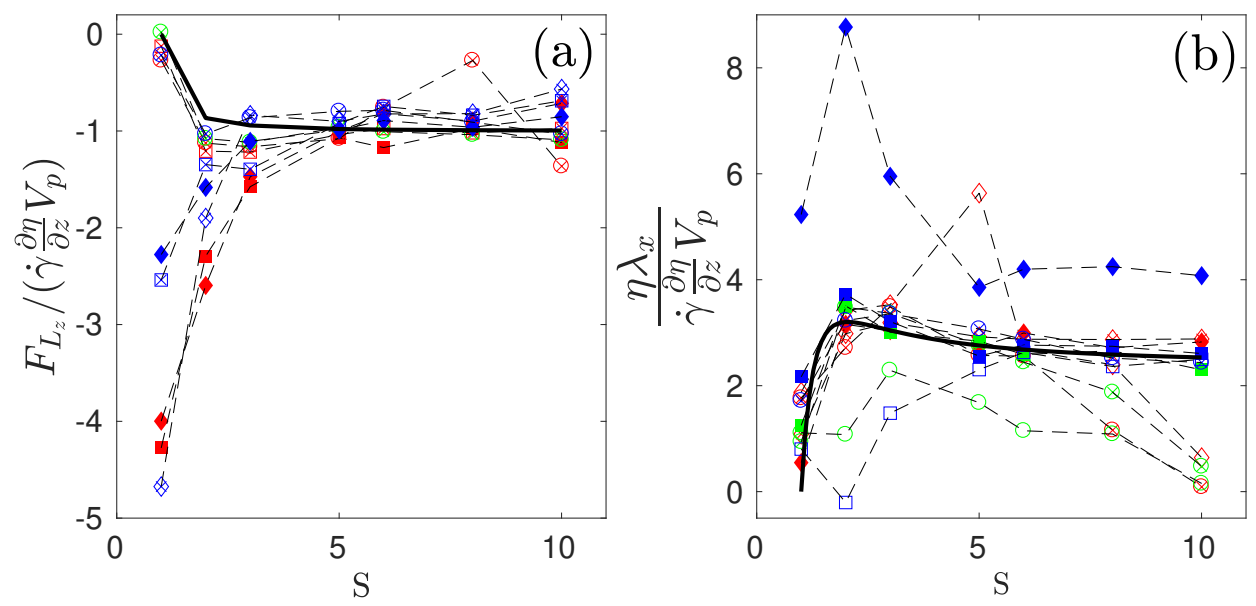

Figure 5.6: (a) Scaled lift forces in $z$-direction of a sheared flow with horizontal gravity and various boundary conditions. Plate velocity $V=\{2.5,5\} \mathrm{m} / \mathrm{s}$, respectively solid and crossed symbols. Plate pressure $P_{0}=$ $\{1,2.5,5\} \mathrm{Pa}$, respectively square, diamond and triangle. Gravitational acceleration $g=\{3,5,7\} \mathrm{m} / \mathrm{s}^{2}$, respectively blue, green and red. (b) Scaled lift force in $x$-direction of a sheared flow with vertical gravity and various boundary conditions. Shear velocity $V=\{1,2.5,5\} \mathrm{m} / \mathrm{s}$, respectively open, closed and crossed symbols. Plate pressure $P_{0}=\{1,2.5,5\} \mathrm{Pa}$, respectively circle, square and diamond. Gravitational acceleration $g=\{3,5,7\} \mathrm{m} / \mathrm{s}^{2}$, respectively blue, green and red. The data has been obtained from Ref. [1].

Simulations of intruders in chute flows show a $z$-independent lift force [21]. This is agreement with the new scaling as for such flows $\dot{\gamma} \frac{\partial \eta}{\partial z}$ is also independent of height, see Appendix B. Simulations in vertical chutes can show reverse segregation when dilute flows are considered [22]. In these cases the segregation mechanism might not apply as the granular flow does not flow in layers. An alternative segregation mechanism in dilute regimes could be due to an emerging density gradient in the flow or just due to the gravity force. Instead, a density gradient emerges which could drive a possible alternative segregation mechanism. Whether the new scaling works in a shear box remains to be seen.

\subsection{FORCE MODEL}

With the newly found scalings in the moderate to large $S$-regime of the lift forces on intruders, see section 5.3, a force model can be developed. The model will be derived for gravity driven flows with only a shear stress component $\dot{\gamma}_{x z}$, such that the following hydrodynamic relations can be adopted.

$$
\frac{\partial p}{\partial z}=\phi \rho_{b} g_{z}
$$

and

$$
\frac{\partial \tau_{x z}}{\partial z}=-\phi \rho_{b} g_{x}
$$



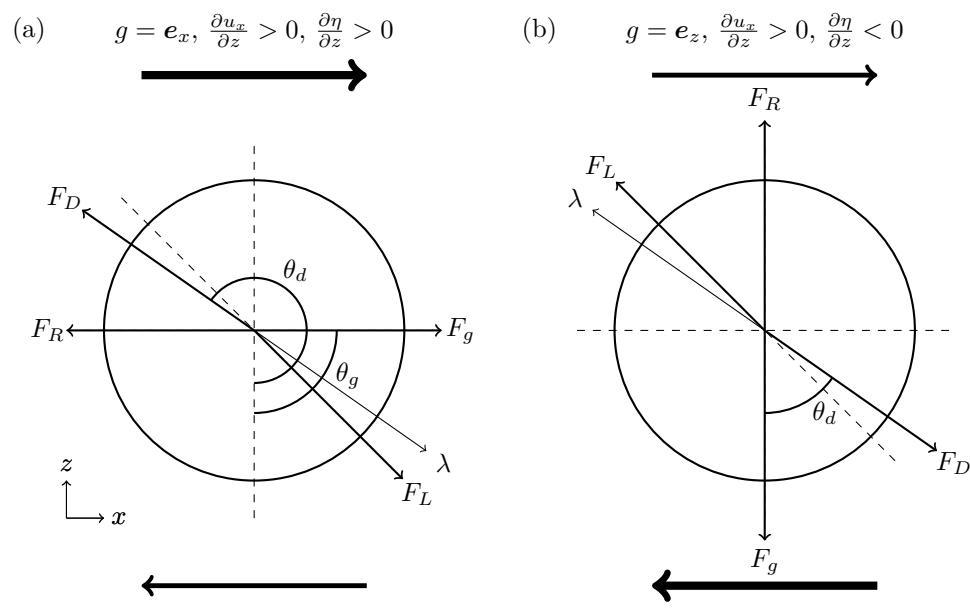

Figure 5.7: A schematic of the forces on an intruder in a sheared flow with (a) horizontal gravity and (b) vertical gravity.

Small anisotropies in dense granular flows are ignored here for simplicity [27].

The model consists of four different force types: a drag force $F_{d}$ related to $\boldsymbol{\lambda}$, an $S$ dependent lift force $F_{L}$, a gravity force $F_{g}$ and a reference force $F_{R}$. Here $F_{R}$ are the forces caused by the presence of stress gradients in the reference flow profile (e.g. buoyancy force). A general schematic description of the force model is shown in Fig. 5.7 for sheared flows with horizontal and vertical gravity, respectively. In the following sections each type of force is discussed.

\subsubsection{REFERENCE FORCES}

Reference forces are the forces on intruders that are already present on particles with $S=1$. Consider the forces on a bulk particle. Since the system is in a (time-averaged) steady state, the total force on a single particle equals zero, $\sum \boldsymbol{F}=\mathbf{0}$. This balance can be expanded into the gravity force $\boldsymbol{F}_{g}$ and the reference forces resulting from collisions with bulk particles $\boldsymbol{F}_{r}$ such that,

$$
\boldsymbol{F}_{g}+\boldsymbol{F}_{r}^{S=1}=\mathbf{0}
$$

with

$$
\boldsymbol{F}_{g}=g \rho_{p} V_{p}\left[\begin{array}{c}
\sin \theta_{g} \\
-\cos \theta_{g}
\end{array}\right]=F_{g}\left[\begin{array}{c}
\sin \theta_{g} \\
-\cos \theta_{g}
\end{array}\right] \text {. }
$$

Next, The contact forces can be expressed as an integral of the stress over the surface of the particle, which has to be proportional to the gravity force,

$$
\int_{A_{p}} \boldsymbol{\sigma}^{\mu, S=1} \boldsymbol{n} d A+\boldsymbol{F}_{g}=\mathbf{0} .
$$


Here $\boldsymbol{\sigma}^{\mu, S=1}$ is the effective microscopic stress felt by the bulk particle and $\boldsymbol{n}$ is the normal outward unit vector. For a bulk particle, it can be shown that

$$
\boldsymbol{\sigma}^{\mu, S=1}=\boldsymbol{\sigma} / \phi
$$

where $\boldsymbol{\sigma}$ is the stress state of the reference flow, see Chapter 3. The most simple approximation of the stress tensor of the considered hydrodynamic model is given by

$$
\boldsymbol{\sigma}=\left[\begin{array}{cc}
-p & \tau \\
\tau & -p
\end{array}\right]
$$

where $p$ and $\tau$ are defined through Eq. (5.6) and Eq. (5.7). This stress tensor has first been used in segregation models by Ref. [12]. Substituting this tensor in Eq. (5.11) and then integrating Eq. (5.10) yields

$$
\boldsymbol{F}^{R, S=1}=\frac{1}{\phi}\left[\begin{array}{c}
\frac{\partial \tau}{\partial z} \\
-\frac{\partial p}{\partial z}
\end{array}\right] V_{p}
$$

Note that this force satisfies Eq. (5.8). The reference force in the $z$-direction is the buoyancy force on the particle and in $z$-direction a similar type of force is observed. By introducing a density ratio $D=\rho_{p} / \rho_{b}$, Eq. (5.13) can be rewritten in terms of the gravity force,

$$
\boldsymbol{F}^{R, S=1}=\frac{1}{D} \boldsymbol{F}_{g},
$$

When the size of the intruder increases, the reference forces have to be corrected due to a change in contact density, see chapter 3 . This is done by applying an $S$-dependent correction function $\phi B(S)$.

$$
\boldsymbol{F}^{R}=-\frac{\phi B}{D} \boldsymbol{F}_{g}
$$

where $\phi B(S=1)=1$. Note that the reference (buoyancy-like) force is always in opposite direction of the gravity force.

\subsubsection{LIFT FORCES}

With the newly obtained scaling, see section 5.3, the lift force can be expressed as

$$
\boldsymbol{F}_{L}=\left[\begin{array}{c}
c_{L_{x}} \\
\operatorname{sign}\left(\dot{\gamma}_{x z}\right) c_{L_{z}}
\end{array}\right] \phi B \dot{\gamma}_{x z} \frac{\partial \eta}{\partial z} V_{p}=\left[\begin{array}{c}
c_{L_{x}} \\
\operatorname{sign}\left(\dot{\gamma}_{x z}\right) c_{L_{z}}
\end{array}\right] F_{L}
$$

where $c_{L_{x}}$ and $c_{L_{z}}$ are positive functions of $S$ and $B$ is the correction due to a lack of scale-separation, see chapter 3 . The direction is automatically satisfied by the shear rate and the sign thereof. The lift force is not present at $S=1$ and hence $c_{L_{z}}(S=1)=0$ and $c_{L_{x}}(S=1)=0$. Since the lift force is related to shear, it is possible that the angle of lift force is $45^{\circ}$. This implies that $c_{L_{z}}=c_{L_{x}}$. However, this is an assumption which will require validation. 


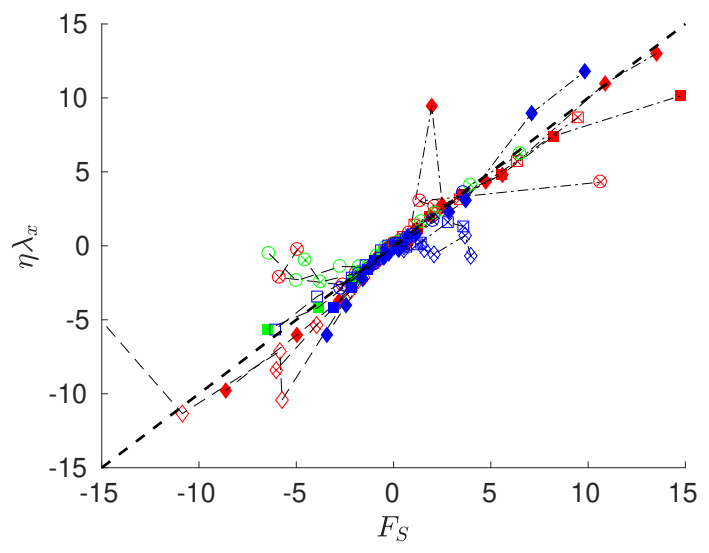

Figure 5.8: The lag velocity times viscosity versus the spring force measured wit vertical gravity, marked by the dashdot lines and horizontal gravity, marked by the dashed lines. Various boundary conditions are: plate velocity $V=\{2.5,5\} \mathrm{m} / \mathrm{s}$, respectively solid and crossed symbols. Plate pressure $P=\{1,2.5,5\} \mathrm{Pa}$, respectively square, diamond and triangle. Gravitational acceleration $g=\{3,5,7\} \mathrm{m} / \mathrm{s}^{2}$, respectively blue, green and red. The data has been obtained from Ref. [1].

\subsubsection{DRAG FORCES}

The presence of a spring in the $z$-direction enables direct lift force measurement. In absence of the spring force, the particle would rise with a (time-averaged) steady velocity [28]. Therefore, the spring force effectively replaces the drag force. This implies that the magnitude of the spring force would be very similar to the magnitude of the drag force in the $z$-direction and additionally that the scaling between $F_{s}$ and $F_{d_{x}}$ are very similar. In Fig. 5.8 a correlation between the spring force and $F_{d_{x}}$ is found implying $F_{s} \propto \eta \lambda_{x}$. As the drag of a single larger intruder particle has not been studied in detail, a Stokesian drag law is adopted here, observed on a dense intruder particle in a chute flow [26].

$$
\boldsymbol{F}_{d}=-c_{d} \phi B \eta \lambda\left[\begin{array}{c}
\sin \theta_{d} \\
-\cos \theta_{d}
\end{array}\right]=-F_{d}\left[\begin{array}{c}
\sin \theta_{d} \\
-\cos \theta_{d}
\end{array}\right],
$$

where $c_{d}$ is the drag coefficient and $\lambda$ is the absolute lag velocity of the particle. The drag coefficient appears to change for different chute flow angles and the exact origin of that behaviour is unknown. Furthermore, it is not clear if the drag coefficient scales with the size ratio. Note that the drag force is always in the opposite direction of the velocity of the lag velocity of the intruder.

\subsubsection{FORCE MODEL}

Summing up all forces discussed yields a model for a single larger intruder in a granular flow,

$$
\left(1-\frac{\phi B}{D}\right) F_{g}\left[\begin{array}{c}
\sin \theta_{g} \\
-\cos \theta_{g}
\end{array}\right]+F_{L}\left[\begin{array}{c}
c_{L_{x}} \\
\operatorname{sign}\left(\dot{\gamma}_{x z}\right) c_{L_{z}}
\end{array}\right]=F_{d}\left[\begin{array}{c}
\sin \theta_{d} \\
-\cos \theta_{d}
\end{array}\right]
$$

The model contains four unknowns, $c_{d}, \theta_{d}, c_{L_{x}}$ and $c_{L_{z}}$. 
From the sheared flow with horizontal gravity, only two functions can be obtained. The lift coefficient $c_{L_{z}}$ is directly obtained as shown in Fig. 5.6(a) and the relation between drag and spring force observed in Fig. 5.8 yields

$$
c_{d} \phi B \frac{\lambda_{z}}{\lambda_{x}}=1
$$

where it is assumed that the magnitude of $F_{s}$ is similar to the drag force in the $z$-direction. The force balance in the $x$-direction does not allow for such measurement due to the absence of a spring force.

Three coefficients can be measured for the sheared flow with vertical gravity. The observed collapse function observed in Fig. 5.6(b) can be written as

$$
\frac{c_{L_{x}}}{c_{d}}=f(S)
$$

where $f(S)$ can be estimated by a 3rd order Laurent series. The second relation is similar to Eq. (5.19). The third coefficient is $c_{L_{z}}$ which can be directly computed by rewriting the force balance in $z$-direction,

$$
c_{L_{z}}=\frac{F_{s}-\left(1-\frac{B}{D}\right) F_{g}}{\eta \frac{\partial \dot{\gamma}}{\partial z} V_{p}} .
$$

Here the Voronoi correction is used, $B=1 / \phi_{p}$, where $\phi_{p}$ is defined as the local Voronoi volume of the intruder [21]. The local Voronoi volumes of the intruder are shown in Fig. 5.9(a). For all simulations they seem to collapse on a single curve of

$$
\phi_{p}(S)=(\phi-1) S^{-1.5}+1,
$$

with $\phi$ the solids fraction of the bulk flow. Note that this functional form is similar to the results found in Ref. [21], with a different power of -1.5 instead of -1.2 , obtained for a 3D chute flow. In Fig. 5.9(b) $c_{L_{z}}$ is shown. Most simulations collapse on a similar curve observed in Fig. 5.6(b). This suggests that that $c_{L_{x}}=c_{L_{z}}$, implying a lift force angle of $45^{\circ}$. Unfortunately, the drag force in the $x$-direction can not be measured and therefore the model can not be closed with the current setup.

Experiments and simulations have reported that reverse segregation can take place for large particles $[29,30]$. The current force model could capture this effect due to the first term in Eq. (5.18), where for increasing $S$ the buoyancy force no longer is strong enough to counter the gravity force. In flows where the lift force is only weak (i.e. small $\dot{\gamma}_{x z} \frac{\partial \eta}{\partial z}$ ) it is possible that the lift force together with the buoyancy force are not strong enough to compensate the gravity force and therefore the particle sinks.

\subsection{CONCLUSIONS AND DISCUSSIONS}

The lift forces on an intruder have been investigated by considering two (plate-driven) sheared systems with a vertical gravity and horizontal gravity. By applying a vertical spring on the intruder, the lift force in the vertical direction can be measured. The lift force in the horizontal direction can only be indirectly observed by measuring a horizontal velocity lag/raise. 

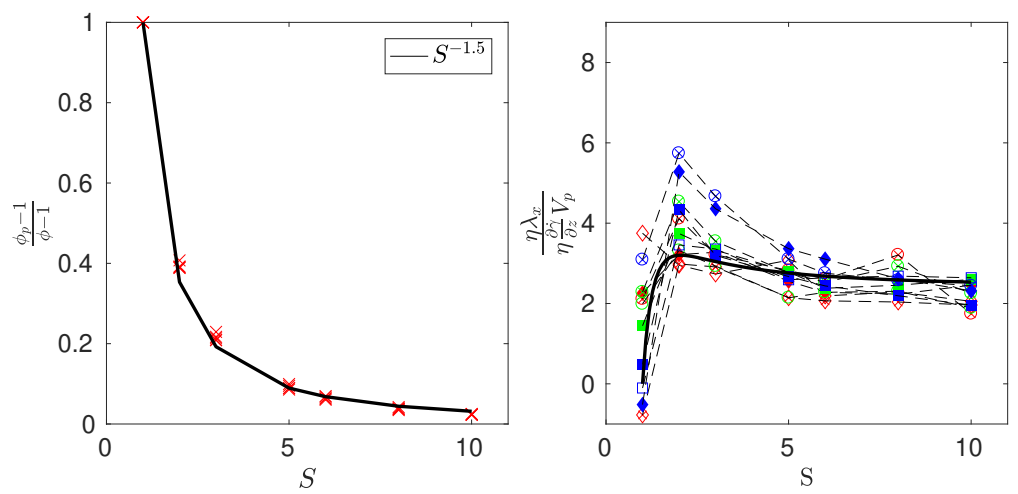

Figure 5.9: (a) Scaled local intruder solids fraction $\phi_{p}$ with $\phi$ for various simulations. (b) Lift coefficient $c_{L_{z}}$, Eq. (5.21) for the system with vertical gravity and different boundary conditions, see Fig. 5.6 for details.

A unique segregation mechanism is uncovered by systematically considering flow properties in many other different configurations. The sign of all flow gradients is computed and compared, revealing a single gradient that determines the direction of segregation, namely the gradient in viscosity $\frac{\partial \eta}{\partial z}$. The implication is that a large intruder particle always moves to low viscosity regions in a flow. This observation is in contrast with the theory of temperature-driven segregation [7]. The viscosity-driven mechanism is confirmed by direct measurements of the lift force in the sheared system, yielding a lift force scaling of $F_{L} \propto \dot{\gamma}_{x z} \frac{\partial \eta}{\partial z} V_{p}$.

The observed scaling implies that $\dot{\gamma}_{x z} \frac{\partial \eta}{\partial z} V_{p}$ is the dominant lift force. However, it remains possible that the (shear stress) related term $\eta \frac{\partial \dot{\gamma}_{x z}}{\partial z} V_{p}$ also induces a weaker lift force with another $S$-dependence. This lift force scaling would show close similarities with Faxen's law [31] and warrants further research. Alternatively, this lift force is related to $\dot{\gamma}_{x z} \frac{\partial \eta}{\partial z}$ by the parameter $\alpha$ and could explain why the lift coefficients measured in both horizontal and vertical sheared systems, see Fig. 5.6(a) and Fig. 5.9(b) scale differently with $S$.

A physical explanation of segregation is that a larger intruder does not "fit" in side a layer of bulk particles, see chapter 4 . Bulk particles have to move around the intruder and this takes considerably more effort in high viscosity regions than in low viscosity regions, yielding an anisotropic and asymmetric stress profile. According to this explanation, segregation could be prevented in flows with no viscosity gradient present. With the presence of gravity this is hard to obtain, but not impossible.

Based on the new segregation scaling, shown in this chapter, a force model is derived that describes the behaviour of an intruder in dense granular flows. The model contains gravity, lift, drag and reference forces (i.e. buoyancy force). The force model takes scaleseparation into account such that for larger $S$ the intruder reference forces reduce. This potentially enables reverse segregation, however more research is required to establish if this is correct [29]. The model could not be closed with the current set of simulations. 
Closure of the model can be obtained by measuring the actual segregation velocity $\lambda_{z}$ when the spring is removed. This would enable the investigation of the $S$-dependence of $c_{d}$. The currently proposed model is expected to be valid only in the dense flow regime.

The current ideas capture the direction of segregation in many systems, but only for the chute and sheared systems the scaling has been directly measured. To fully confirm the proposed mechanism, it is necessary to perform single intruder simulations in vertical chutes, shear box setups and flows with no viscosity gradients.

\section{REFERENCES}

[1] F. Guillard, Y. Forterre, and O. Pouliquen, Scaling laws for segregation forces in dense sheared granular flows, Journal of Fluid Mechanics 807, R1 (2016).

[2] J. McCarthy, Turning the corner in segregation, Powder Technology 192, 137 (2009).

[3] J. M. N. T. Gray, Particle segregation in dense granular flows, Annual Review of Fluid Mechanics 50, 407 (2018).

[4] A. M. Scott and J. Bridgwater, Interparticle percolation: A fundamental solids mixing mechanism, Industrial \& Engineering Chemistry Fundamentals 14, 22 (1975).

[5] S. B. Savage and C. K. K. Lun, Particle size segregation in inclined chute flow of dry cohesionless granular solids, Journal of Fluid Mechanics 189, 311 (1988).

[6] Y. Fan and K. M. Hill, Phase transitions in shear-induced segregation of granular materials, Physical Review Letters 106, 218301 (2011).

[7] Y. Fan and K. M. Hill, Theory for shear-induced segregation of dense granular mixtures, New Journal of Physics 13, 095009 (2011).

[8] J. M. N. T. Gray and A. R. Thornton, A theory for particle size segregation in shallow granular free-surface flows, in Proceedings of the Royal Society of London A: Mathematical, Physical and Engineering Sciences, Vol. 461 (The Royal Society, 2005) pp. 1447-1473.

[9] P. Gajjar and J. M. N. T. Gray, Asymmetric flux models for particle-size segregation in granular avalanches, Journal of Fluid Mechanics 757, 297 (2014).

[10] K. van der Vaart, P. Gajjar, G. Epely-Chauvin, N. Andreini, J. M. N. T. Gray, and C. Ancey, Underlying asymmetry within particle size segregation, Physical Review Letters 114, 238001 (2015).

[11] D. R. Tunuguntla, O. Bokhove, and A. R. Thornton, A mixture theory for size and density segregation in shallow granular free-surface flows, Journal of fluid mechanics 749, 99 (2014).

[12] B. Marks, P. Rognon, and I. Einav, Grainsize dynamics of polydisperse granular segregation down inclined planes, Journal of Fluid Mechanics 690, 499 (2012).

[13] Y. Fan and K. Hill, Theory for shear-induced segregation of dense granular mixtures, New journal of physics 13, 095009 (2011). 
[14] B. Marks, J. A. Eriksen, G. Dumazer, B. Sandnes, and K. J. Måløy, Size segregation of intruders in perpetual granular avalanches, Journal of Fluid Mechanics 825, 502 (2017).

[15] D. R. Tunuguntla, T. Weinhart, and A. R. Thornton, Comparing and contrasting sizebased particle segregation models, Computational Particle Mechanics 4, 387 (2017).

[16] D. R. Tunuguntla and A. R. Thornton, Balancing size and density segregation in bidisperse dense granular flows, in EPJ Web of Conferences, Vol. 140 (EDP Sciences, 2017) p. 03079.

[17] J. Guo, Motion of spheres falling through fluids, Journal of Hydraulic Research 49, 32 (2011).

[18] S. Liu and J. J. McCarthy, Transport analogy for segregation and granular rheology, Physical Review E 96, 020901 (2017).

[19] A. Tripathi and D. V. Khakhar, Numerical simulation of the sedimentation of a sphere in a sheared granular fluid: a granular stokes experiment, Physical Review Letters 107, 108001 (2011).

[20] C. Kloss, C. Goniva, A. Hager, S. Amberger, and S. Pirker, Models, algorithms and validation for opensource dem and cfd-dem, Progress in Computational Fluid Dynamics, an International Journal 12, 140 (2012).

[21] K. van der Vaart, M. P. van Schrojenstein Lantman, T. Weinhart, S. Luding, C. Ancey, and A. R. Thornton, Segregation of large particles in dense granular flows suggests a granular saffman effect, Physical Review Fluids 3, 074303 (2018).

[22] Y. Fan and K. M. Hill, Phase transitions in shear-induced segregation of granular materials, Physical review letters 106, 218301 (2011).

[23] GDR-MiDi, On dense granular flows, The European Physical Journal E 14, 341 (2004).

[24] P. Jop, Y. Forterre, and O. Pouliquen, A constitutive law for dense granular flows, Nature 441, 727 (2006).

[25] P. C. Johnson and R. Jackson, Frictional-collisional constitutive relations for granular materials, with application to plane shearing, Journal of fluid Mechanics 176, 67 (1987).

[26] A. Tripathi and D. V. Khakhar, Density difference-driven segregation in a dense granular flow, Journal of Fluid Mechanics 717, 643-669 (2013).

[27] T. Weinhart, R. Hartkamp, A. R. Thornton, and S. Luding, Coarse-grained local and objective continuum description of three-dimensional granular flows down an inclined surface, Physics of Fluids 25, 070605 (2013).

[28] L. Staron, Rising dynamics and lift effect in dense segregating granular flows, Physics of Fluids 30, 123303 (2018). 
[29] N. Thomas and U. D'ortona, Evidence of reverse and intermediate size segregation in dry granular flows down a rough incline, Physical Review E 97, 022903 (2018).

[30] N. Thomas, Reverse and intermediate segregation of large beads in dry granular media, Physical Review E 62, 961 (2000).

[31] E. Guazzelli, J. F. Morris, and S. Pic, A Physical Introduction to Suspension Dynamics, Cambridge Texts in Applied Mathematics (Cambridge University Press, 2011). 



\section{6}

\section{CONTINUUm Simulations OF}

Granular Media in a

\section{Split-Bottom Ring Shear Cell}

Recently proposed generalised $\mu(I)$-rheologies are compared to the classical $\mu(I)$-rheology for steady state granular flow. This is done by modifying the viscosity of the Navier-Stokes equations and solving them using the finite element method. For verification of the solver, a 2D Couette shear cell benchmark is used, while for validation a split-bottom ring shear cell geometry is used as it exhibits a shear band with a wide range of stress and strain states. Compared to the classical $\mu(I)$-rheology the generalised rheologies have additional corrections for (i) particle stiffness, (ii) surface effects and (iii) low inertial numbers. The stiffness has a minor effect on the centre of the shear band, shifting it away from the centre a little. The surface correction shows a more profound qualitative effect, so far not seen in experiments, which could be related to particle friction. The low inertial flow correction shows some improvement of the shear band tails compared to the classical $\mu(I)$-rheology and removes the need for an arbitrary regularisation for $I \rightarrow 0$, however further, corrections are identified as necessary. Recommendations are given to investigate effects of granular temperature and compressibility on the generalised rheology.

\subsection{INTRODUCTION}

Industry is heavily based on equipment dealing with granular media such as chute flows, rotating drums and screw conveyors. It would be of impeccable value if the designs for these equipment could be done solely on a computer, avoiding costly experiments and prototyping.

The current state-of-the-art simulation methods for granular matter are discrete particle methods (DPM) [1]. These simulations can accurately capture a wide variety of phenomenon seen in granular media such as force chains, segregation, dilation and jamming of the material [2-4]. A disadvantage of DPM is the limit of the number of particles 
of a simulation, as the computational effort scales, in the best case, with the number of particles. One way to overcome this limit is to massively parallelise the DPM algorithm, see Appendix B. Handling the shear amount of data generated by a DPM simulation is challenging. A useful method is to convert particle data into continuum fields by using coarse graining (CG) analysis [5]. The fields obtained by CG give good insight in the dynamics of a granular system, yet its applicability to optimisation methods for industrial equipment is non-trivial.

Another way of simulating granular materials is using continuum methods in which a system of Partial Differential Equations (PDE's) is solved numerically, enabling a wide variety of new applications and methods. One of the benefits of this approach is the ability to solve large-scale industrial problems, since not every single particle is tracked, but a continuum is approximated. Another major advantage is that gradient-based optimisation techniques, such as adjoint-based methods [6], can be applied to create cost and energy efficient equipment designs.

Simulating granular materials by continuum equations is a challenging subject, i.e. see [7], since the governing PDE's and their closure constitutive relations (i.e. rheology) are still not well understood. Much progress has been made the past two decades. One of the major milestones is the $3 \mathrm{D}$ chute flow simulations with frictional walls [8]. More recently column collapse, [9], flow over cylinders $[10,11]$ and more complex geometries [12] have also been captured by continuum models.

Since the quest for the granular rheology is still in progress, it is no surprise all these simulations use different rheologies, with the Schaeffer rheology, Tardos rheology and $\mu(I)$-rheology being the most popular ones [8, 13, 14]. In this work we focus on the latter. This model relates the shear rate and shear stress in a single point by a granular viscosity, resembling a yield stress fluid. In the limit of dry, frictional rigid particles, it is governed by a single parameter, the inertial number $I$, the ratio of the shear rate forces and the confining pressure forces. A recent proposed extension also takes particle stiffness and low $I$ correction into account [15]. An alternative extension of the $\mu(I)$-rheology is the 'nonlocal' rheology, where a new state variable, the fluidity, is defined, based on a certain relation between the shear rate and the shear stress [16]. The evolution of the fluidity is governed by an additional differential equation which reduces to the local $\mu(I)$-rheology at high inertial numbers and to the Helmholtz equation at low inertial numbers. This model has been successfully applied to split-bottom shear cell geometries [17].

The generalised $\mu(I)$-rheology [15] has not yet been verified with continuum methods. This chapter is focussed on solving the fluid equations using this rheology. The simulation setup uses a split-bottom ring shear cell geometry. This geometry is chosen as it exhibits a rich variety in stresses and shear rates within one setup [18]. Moreover, the generalised rheology has been developed within this geometry.

The split-bottom ring shear cell induces a wide shear band in the bulk of the granular flow, while in the standard Couette cell one has a local shear band at the wall. This was observed first by experiments [19], where the top of a split-bottom shear cell was investigated. The setup contains regions with very low inertial number and therefore it is expected that the classical $\mu(I)$-rheology does not fully describe the granular problem. The confining stress varies from low values at the surface to high values at the bottom and thus also has to be considered, in particular for soft particles. This extended $\mu(I)$ - 


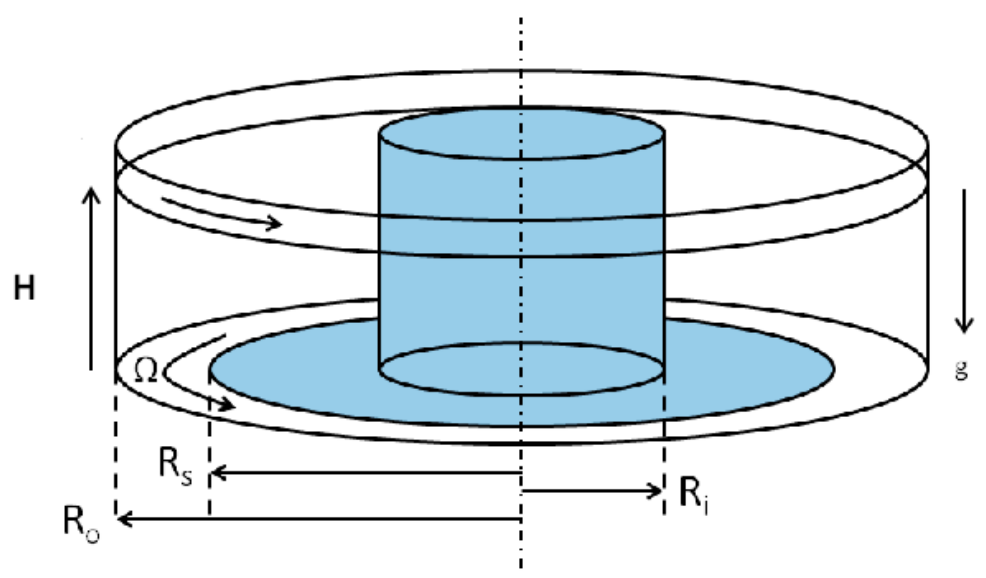

Figure 6.1: Schematic overview of the split-bottom ring shear cell setup (created by A. Singh). Definitions and values of the symbols are given in Table 6.1.

rheology is also investigated [15], involving additional non-dimensional numbers based on the stiffness stress of the particle.

The orginisation of the chapter is as follows. In section 6.2 the geometry of the simulation is described together with the governing equations and the rheology. In section 6.3 the continuum solver is explained in detail including possible regularisations. The results obtained from the model are discussed in section 6.4 and finally the conclusion and discussion is given in section 6.5

\subsection{Simulation Model}

The simulation model consists of a geometry, governing equations with boundary equations and a rheology for the fluid viscosity. In section 6.2.1 the geometry of the splitbottom ring shear cell is discussed. This includes a short overview of the observations found in experiments and DPM simulations. The governing equations of the continuum model and boundary conditions are presented in section 6.2.2. Three granular rheologies are discussed in section 6.2.3 and will be compared with experiments and DPM simulations in section 6.4.

\subsubsection{GEOMETRY}

The geometry of the split-bottom ring shear cell is shown in Fig. 6.1. It consists of an inner cylinder of radius $R_{i}$ and an outer cylinder of radius $R_{o}$. The bottom of the shear cell is split between the inner and outer cylinder at split radius $R_{s}$. The fill height of the split-bottom ring shear cell is $H$. The inner cylinder is fixed and the outer cylinder is rotating with an angular velocity of $\Omega$. Dimensional values of the geometry setup can be found in Table 6.1 and are similar to the DPM simulation geometry in [15].

The system is axi-symmetric with only an azimuthal velocity component $u_{\phi}$. The 


\begin{tabular}{l|l|l|l}
\hline Parameter & Symbol & value & unit \\
\hline Inner radius & $R_{i}$ & 0.0147 & {$[\mathrm{~m}]$} \\
Outer radius & $R_{O}$ & 0.11 & {$[\mathrm{~m}]$} \\
Split radius & $R_{S}$ & 0.085 & {$[\mathrm{~m}]$} \\
Filling height & $H$ & $0.01-0.08$ & {$[\mathrm{~m}]$} \\
Angular frequency & $\Omega$ & 0.01 & {$[\mathrm{rad} / \mathrm{s}]$} \\
\hline
\end{tabular}

Table 6.1: Geometrical properties of the split-bottom ring shear cell. Dimensions of the setup follow [15].

non-dimensionalised angular velocity is defined as $\omega(r)=u_{\phi} /(2 \pi r \Omega)$. The profile of $\omega(r)$ depends on the ratio of the fill height with split radius $H / R_{s}$. At the surface $\omega(r)$ evolves from a step function at $H / R_{s} \rightarrow 0$ to a shear band with a certain width $W_{\text {top }}$ and a band centre $R_{c_{t o p}}$. From experiments it has been observed that the shear band slowly moves towards the inner cylinder, while the width remains constant. For $H / R_{s}<0.45$ the angular rotation in these experiments is captured well by an error function [20],

$$
\omega_{\text {top }}(r)=\frac{1}{2}+\frac{1}{2} \operatorname{erf}\left(\lambda_{s}\right),
$$

which is symmetric around $R_{c}$ and described by the coordinate

$$
\lambda_{s}=\frac{r-R_{c}}{W_{\text {top }}},
$$

where $r$ is the radial coordinate. Experiments in this regime $R_{s}$ and $W_{\text {top }}$ show that $R_{c}$ and $W_{\text {top }}$ scale accordingly,

$$
\left(R_{s}-R_{c_{t o p}}\right) / R_{s}=\left(H / R_{s}\right)^{5 / 2}
$$

and

$$
W_{\text {top }} / d \sim(H / d)^{2 / 3}
$$

Given a ratio $H / R_{s}$, the width $W$ and shear center $R_{c}$ change as functions of $z$. DPM simulations [21] have shown that the width behaves like,

$$
W(z)=W_{\text {top }} \sqrt{1-\left(1-\frac{z}{H}\right)^{2}} .
$$

As the ratio of $H / R_{s}$ increases the angular velocity profile becomes asymmetric. It however still can be represented by an error function [20],

$$
\omega_{\text {top }}(r)=\frac{1}{2}+\frac{1}{2} \operatorname{erf}(\lambda)
$$

with an asymmetric coordinate

$$
\lambda=a_{0}+a_{1} r+a_{2} r^{3}
$$




\subsubsection{GOVERNING EQUATIONS}

The granular fluid in a split-bottom ring shear cell is modeled by the incompressible steady state axi-symmetric Navier-Stokes equations. Here incompressibility is assumed for simplicity. The axi-symmetric coordinates $(r, z, \theta)$ are respectively in the radial, axial and azimuthal direction. The assumption in the axi-symmetric coordinates is that all derivatives with respect to $\theta$ are not present. This implies that all variables in the $\theta$ direction are constant and the 3D equations can be solved in the $2 \mathrm{D} z-r$-plane. The steady state momentum equations are,

$$
\begin{gathered}
\rho\left[u_{r} \frac{\partial u_{r}}{\partial r}-\frac{u_{\theta}^{2}}{r}+u_{z} \frac{\partial u_{r}}{\partial z}\right]=-\frac{\partial p}{\partial r}+\frac{\partial \tau_{r r}}{\partial r}+\frac{\tau_{r r}}{r}-\frac{\tau_{\theta \theta}}{r}+\frac{\partial \tau_{r z}}{\partial z}, \\
\rho\left[u_{r} \frac{\partial u_{z}}{\partial r}+u_{z} \frac{\partial u_{z}}{\partial z}\right]=\rho g-\frac{\partial p}{\partial z}+\frac{\partial \tau_{z r}}{\partial r}+\frac{\tau_{z r}}{r}+\frac{\partial \tau_{z z}}{\partial z} \\
\rho\left[u_{r} \frac{\partial u_{\theta}}{\partial r}+\frac{u_{r} u_{\theta}}{r}+u_{z} \frac{\partial u_{\theta}}{\partial z}\right]=\frac{\partial \tau_{\theta r}}{\partial r}+\frac{\tau_{\theta r}}{r}+\frac{\tau_{r \theta}}{r}+\frac{\partial \tau_{\theta z}}{\partial z} .
\end{gathered}
$$

Here $u_{r}, u_{z}$ and $u_{\theta}$ are the radial, axial and azimuthal velocity, respectively, $p$ is the fluid pressure and $\tau$ is the (deviatoric) shear stress tensor. The gravitational acceleration $g$ only acts in the $z$-direction. The conservation of mass is satisfied by the continuity equation,

$$
\frac{\partial u_{r}}{\partial r}+\frac{u_{r}}{r}+\frac{\partial u_{z}}{\partial z}=0
$$

The shear stress tensor $\tau_{i j}$ is defined as

$$
\tau_{i j}=2 \eta \gamma_{i j}
$$

where $\gamma_{i j}$ are the elements of the shear rate tensor given by

$$
\dot{\boldsymbol{r}}=\left[\begin{array}{ccc}
\frac{\partial u_{r}}{\partial r} & \frac{1}{2}\left[\frac{\partial u_{r}}{\partial r}+\frac{\partial u_{z}}{\partial r}\right] & \frac{1}{2}\left[\frac{\partial u_{\phi}}{\partial r}-\frac{u_{\phi}}{r}\right] \\
\frac{1}{2}\left[\frac{\partial u_{r}}{\partial r}+\frac{\partial u_{z}}{\partial r}\right] & \frac{\partial u_{z}}{\partial z} & \frac{1}{2} \frac{\partial u_{\phi}}{\partial z} \\
\frac{1}{2}\left[\frac{\partial u_{\phi}}{\partial r}-\frac{u_{\phi}}{r}\right] & \frac{1}{2} \frac{\partial u_{\phi}}{\partial z} & \frac{u_{r}}{r}
\end{array}\right] .
$$

The viscosity $\eta$ is defined by the granular rheology, see section 6.2 .3 . The norm of the shear rate tensor is given by

$$
|\dot{\gamma}|=\sqrt{\gamma_{i j} \gamma_{i j}}
$$

Note that Eq. (6.12) assumes that $\boldsymbol{\tau}$ and $\dot{\boldsymbol{\gamma}}$ are co-linear.

No-slip boundary conditions are applied to the walls. The axial and radial velocities are always set to zero, $u_{z}=u_{r}=0$. The azimuthal velocities are give by

$$
\begin{gathered}
u_{\theta}\left(r=R_{i}, z\right)=0, \\
u_{\theta}(r, z=0)= \begin{cases}r<R_{s}, & 0 \\
r \geq R_{s}, & \omega r\end{cases}
\end{gathered}
$$




$$
u_{\theta}\left(r=R_{o}, z\right)=\omega R_{o},
$$

The free surface is modeled as a traction free boundary,

$$
\boldsymbol{\sigma} \cdot \boldsymbol{n}_{s}=\mathbf{0}
$$

Here $\boldsymbol{\sigma}$ is the total stress tensor and $\boldsymbol{n}_{\boldsymbol{s}}$ is the outward normal vector of the surface, i.e. $\boldsymbol{n}_{\boldsymbol{s}}=\boldsymbol{e}_{z}$

\subsubsection{RHEOLOGY}

To complete the governing equations in section 6.2.2 closure relation for $\eta$ in equation Eq. (6.12) has to be provided. For the $\mu(I)$-rheology family the viscosity is defined as

$$
\eta=\frac{\mu p}{|\dot{\gamma}|}
$$

where the bulk friction $\mu$ is a function that depends on the specifically chosen rheology. The classical $\mu(I)$-rheology describes the friction $\mu$ in Eq. (6.19) by a phenomenological expression based on the inertial number $I=\frac{|\dot{\gamma}| d_{p}}{\sqrt{p / \rho}}$. Experimental results [22] show that

$$
\mu(I)=\mu_{s}\left[1+\frac{\Delta \mu / \mu_{s}}{I_{0} / I+1}\right],
$$

where $\mu_{s}, \Delta \mu$ and $I_{0}$ are material properties. The validity of this rheology lies in the moderately inertial regime with inertial number in the range of $10^{-3}<I<1$, depending on the material parameters. The flow is in the quasi-static regime $\left(I<10^{-3}\right)$, outside the shear band, where the $\mu(I)$-rheology is ill-posed [23]. An extended model has been proposed in [15] adding an empirical stretched exponential [24],

$$
\mu_{e}(I)=\mu(I)\left[1-e^{-\left(\frac{I}{I^{*}}\right)^{\alpha}}\right]
$$

Here $I^{*}$ is a new material parameter, but possible dependencies on other fields are neglected here. The exponential ensures that $\lim _{I \rightarrow 0} \frac{\mu}{\dot{\gamma}}<\infty$ in similar spirit as the rheology proposed in [25].

Although $I$ is the dominating parameter of the $\mu(I)$-rheology, other non-dimensional parameters have been investigated [15]. The softness number $p_{k}$ is defined as the ratio of pressure forces and the stiffness related to elastic forces between particles,

$$
p_{k}=\frac{p d_{p}}{k},
$$

where $k$ is the stiffness of the particle. Furthermore, the surface number $p_{g}$ is defined as the ratio of pressure forces and gravity forces,

$$
p_{g}=\frac{6 p}{\pi \rho_{p} d_{p} g},
$$


where $g$ is the gravitational acceleration. As $p \propto \rho_{p} g(H-z)$ this number essentially is height of the flow divided by the particle diameter. Close to the free surface of the flow $p_{g}$ becomes important. The general rheology with these parameters is

$$
\mu_{f}\left(I, p_{k}, p_{g}\right)=\mu_{e}(I)\left[1-\left(\frac{p_{k}}{p_{0}}\right)^{\beta}\right]\left[1-a^{\prime} e^{-\frac{p_{g}}{p_{g}}}\right] .
$$

Here $p_{0}, \beta, a^{\prime}$ and $p_{g 0}$ are material parameters. Unless otherwise stated the material parameters are given by Table 6.2. These material parameters were obtained from DPM simulations using a mean particle diameter of $d_{b}=0.0022[\mathrm{~mm}]$, a particle density $\rho_{b}=$ $2000\left[\mathrm{~kg} / \mathrm{m}^{3}\right]$, a solids fraction of $\phi=0.64$ and a particle stiffness of $k_{p}=120$ [N/m] [15].

\begin{tabular}{|c|c|c|}
\hline rheology & coefficient & value \\
\hline$\mu(I)$ & $\mu_{s}$ & 0.16 \\
& $\Delta \mu$ & 0.24 \\
& $I_{0}$ & 0.07 \\
\hline$\mu_{e}(I)$ & $\alpha$ & 0.48 \\
& $I^{*}$ & $4.85 \times 10^{-5}$ \\
\hline$\mu_{f}\left(I, P_{k}, P_{g}\right)$ & $\beta$ & 0.5 \\
& $p_{0}^{*}$ & 0.90 \\
& $a^{\prime}$ & 0.75 \\
& $p_{g 0}^{*}$ & 2.30 \\
\hline
\end{tabular}

Table 6.2: Rheology parameters for the corresponding rheologies in Eq. (6.20), Eq. (6.21) and Eq. (6.24). Values adopted from [15].

\subsection{MeTHODS}

The granular rheology introduces non-linear behaviour w.r.t. $\dot{\gamma}$ and $p$. Recent work has focussed on benchmarking a Couette cell in 2D with a simplified $\mu(I)$-rheology [14]. In these simulations the pressure was taken as constant as many standard solvers have problems with the pressure dependence of the $\mu(I)$-rheology. Often incompressible fluid solvers use a segregated solution procedure based on the Semi-IMplicit Pressure Linked Equations (SIMPLE) algorithm [26]. The SIMPLE algorithm is an iterative procedure that does not solve the continuity equation but a pressure Poisson equation instead. This pressure equation is not yet adapted to granular rheologies. In order to tackle the pressure dependency of the rheology, a monolithic approach is taken here, where the pressure acts as a Lagrange multiplier for the continuity equation.

The open-source physics library oomph-lib (http:/ /www.oomph-lib.org, [27]) is used to solve the set of equations with a Finite Element Method (FEM) approach. The solution method is discussed in section 6.3.1 and regularisations and modifications necessary for the granular rheology and discussed in section 6.3.2 


\subsubsection{SOLUTION METHOD}

A standard Galerkin method is used to approximate the solution to the physical problem. The simulation domain is tessellated into rectangular elements. Within an element the solution of a state variable $u(x)$ is approximated as

$$
u_{h}\left(x_{i}\right)=\sum_{j=1}^{M} U_{j} \psi_{j}\left(x_{i}\right),
$$

where $i$ is an integration point of the element, $\psi_{j}$ is a basis function in this element and $M$ is the number of basis functions used to approximate $u(x)$. The elements used in this setup are rectangular Taylor-Hood elements [28]. The velocity is approximated by bi-quadratic basis functions $(M=9)$ and the pressure bi-linear $(M=4)$. The vector of unknowns within an element is denoted as $\boldsymbol{U}$.

Using the weak (integral) formulation, a residual vector $\boldsymbol{R}(\boldsymbol{U})$ can be derived such that the approximated solution is the exact solution if

$$
\boldsymbol{R}(\boldsymbol{U})=\mathbf{0} .
$$

Due to the non-linear behaviour of the Navier-Stokes equations this system can not be solved directly and a Newton-iteration method is used. For a given set of unknowns $\boldsymbol{U}^{n}$ at iteration $n$, an updated solution can be computed,

$$
\boldsymbol{U}^{n+1}=\boldsymbol{U}^{n}+\delta \boldsymbol{U} .
$$

Here $\delta \boldsymbol{U}$ is the increment. The increment is calculated by solving the following system of equations

$$
\boldsymbol{J} \delta \boldsymbol{U}=-\boldsymbol{R}\left(\boldsymbol{U}^{n}\right),
$$

with the Jacobian matrix

$$
\boldsymbol{J}=\frac{\partial \boldsymbol{R}}{\partial \boldsymbol{U}} .
$$

Calculation of $\boldsymbol{J}$ is done analytically, with possibly a regularisation to accommodate the unusual rheology as discussed in section 6.3.2.

The split-bottom shear cell geometry has a discontinuity at the split. For accurate solutions a non-uniform grid is generated such that there is improved accuracy at the split. Adaptive mesh generation in oomph-lib is performed using a spatial error estimator which estimates if an element has to merge with another element or if they have to split up. For the current problem the Navier-Stokes equations are first solved using a constant viscosity of unity, with up to 6 grid refinements. After the final level grid is obtained, the viscosity is changed from constant viscosity. The solution on the finest grid is used as initial guess for the solution of the full rheology.

\subsubsection{REGULARISATION}

The classical $\mu(I)$-rheology requires a regularisation for the regions where the shear rate approximates zero, as Eq. (6.19) diverges. Many regularisation techniques have been proposed in the literature such as e.g. in Ref. [10]. Here the regularisation of Ref. [14] is 
adopted such that a comparison can be made with the benchmark results. The regularisation replaces Eq. (6.14) with

$$
|\dot{\gamma}|=\sqrt{\gamma_{i j} \gamma_{i j}+\epsilon_{\dot{\gamma}}^{2}}
$$

where $\epsilon=\sqrt{\epsilon_{\dot{\gamma}}^{2} / \Omega^{2}}$ is the non-dimensional regularisation parameter. Note that $\mu_{e}(I)$ and $\mu_{f}\left(I, p_{k}, p_{g}\right)$ do not require such regularisation.

When computing the Jacobian, Eq. (6.29), precautions have to be taken in order to ensure that the terms $\frac{\partial \eta}{\partial|\dot{\gamma}|}$ and $\frac{\partial \eta}{\partial p}$ do not diverge. To ensure the Newton-method from converging, these divergent derivatives are set to zero, yielding a slow but robust convergence. Convergence could be improved by implementing weighting strategies for these terms [29].

Pressure at the surface of a granular flow generally should vanish if there is no force applied. However, to avoid zero pressure, a small pressure limit is implemented $p_{\text {reg }}=$ $p+p_{\epsilon}$ with $p_{\epsilon}=1.0 \times 10^{-8}$.

\subsubsection{BENCHMARK}

To verify the implementation of the rheology, the solver is compared to the recent benchmark of the $\mu(I)$-rheology [14]. In this benchmark a standard Couette shear cell is simulated with a moving inner wall and static outer wall. The benchmark test uses a 2D setup simulating the whole shear cell. Here we take the axi-symmetric approach so the numerical performances can not be compared, however it serves as a good validation tool to see if the rheology is implemented correctly. In Fig 6.2 the results of this benchmark test are shown for a grid of $n_{r}=40, n_{z}=20, n_{r}=40, n_{z}=10$ and a grid for $n_{r}=80$, $n_{z}=20$. Note that all grids collapse very well on the benchmark curves, except around
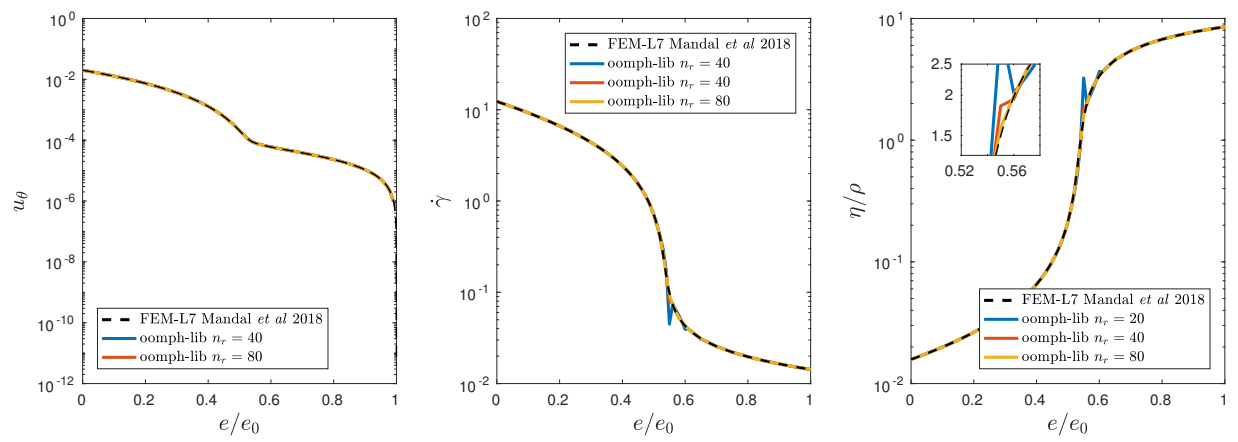

Figure 6.2: Velocity profile, shear rate and viscosity as function of the standard shear cell coordinate from the inner boundary to the outer boundary. Results are compared with a benchmark test.

$\epsilon / \epsilon_{0}=0.5$. The inset in Fig. 6.2 shows a small discrepancy, which is resolved as the grid is refined. As the split-bottom ring shear cell is in the same regime of $I$ as the benchmark it is concluded that a grid with $n_{r}=80$ is sufficient. 


\subsection{RESULTS}

Before the various granular rheologies can be compared, the best possible regularisation parameter needs to be determined for the classical $\mu(I)$-rheology. Simulations for different parameters of $\epsilon_{\dot{\gamma}}$ are performed with $H / R_{S}=0.24$, the symmetric regime. The resulting function $\omega_{\text {top }}(r)$ is fitted with Eq. (6.1) yielding the symmetric coordinate $\lambda_{S}$, Eq. (6.2), and the numerical equivalent is

$$
\lambda=\operatorname{erf}^{-1}\left(2 \omega_{t o p}-1\right) .
$$

The results for various $\epsilon_{\dot{\gamma}}$ are shown in Fig. 6.3. For reasonably small values of $\epsilon_{\dot{\gamma}}$ the shear band is captured well. In the tails $\epsilon_{\dot{\gamma}}$ plays an important role, but none of the values can capture the tails accurately. The best result is obtained with values close to $\epsilon_{\dot{\gamma}} \approx$ $1.0 \times 10^{-2}$. Larger values show solutions that have no resemblance with an error function and smaller values show over/undershoot. All simulations henceforth use $\epsilon_{\dot{\gamma}}=7.0 \times 10^{-3}$ for the classical $\mu(I)$-rheology.

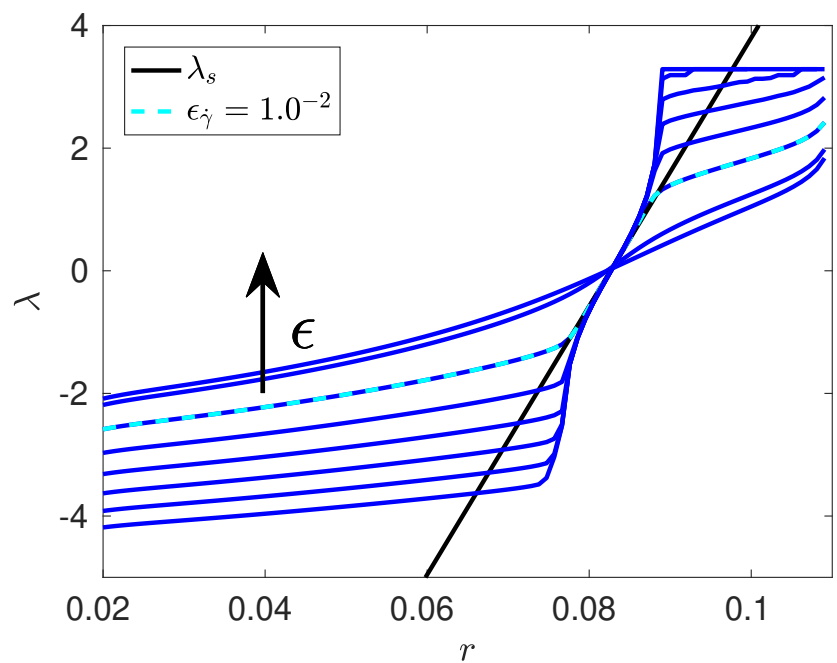

Figure 6.3: Symmetric coordinate at $H / R_{s}=0.24$ for various regularisation values of $\epsilon_{\dot{\gamma}}=1.0^{-\alpha}$ with $\alpha=$ $1,2, \ldots, 7 . \lambda_{s}$ is obtained by fitting $\omega_{\text {top }}$. The black line corresponds to Eq. (6.1).

For all rheologies the angular velocity of the left-side of the shear band is shown in Fig. 6.4 together with corresponding symmetric fit functions. The rheologies appear to be very comparable, capturing the centre of the shear band, but the tails of the shear band show clear deviation from the error function. This can be seen more clearly by plotting $\lambda$, see Fig. 6.5. Surprisingly, the best possible regularisation for the classical $\mu(I)$-rheology is very comparable to $\mu_{e}(I)$. The regularisation of the $\mu(I)$-rheology is not physically based, but the $\mu_{e}(I)$-rheology is inspired by DPM simulations and has a more physical base. All presented rheologies are lacking the physical mechanism that can capture the tails, but the shear band is captured well by all. 


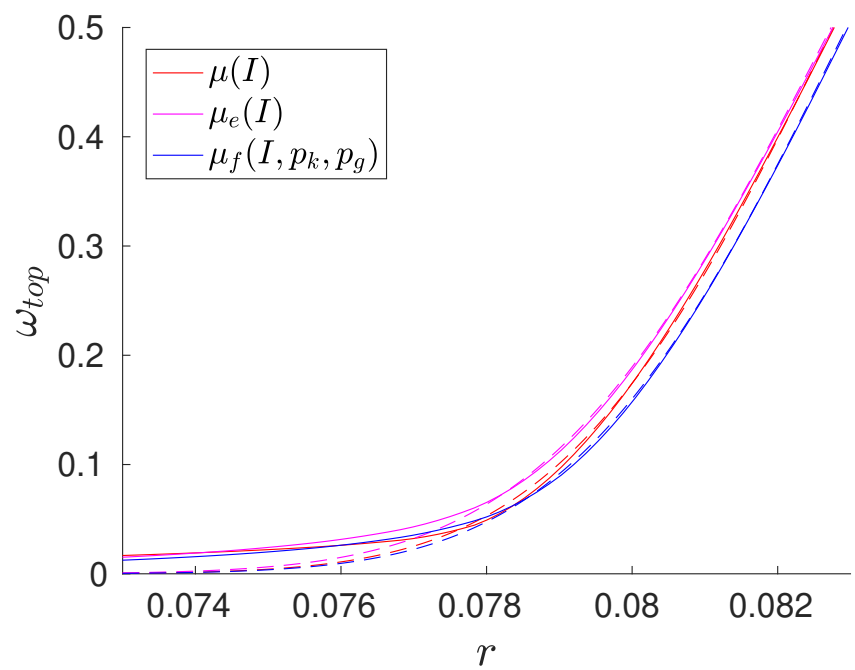

Figure 6.4: Left side of the shear band for different rheologies with $H / R_{s}=0.24$. Dashed lines are obtained by fitting $\omega_{\text {to }}$ to Eq. (6.1).

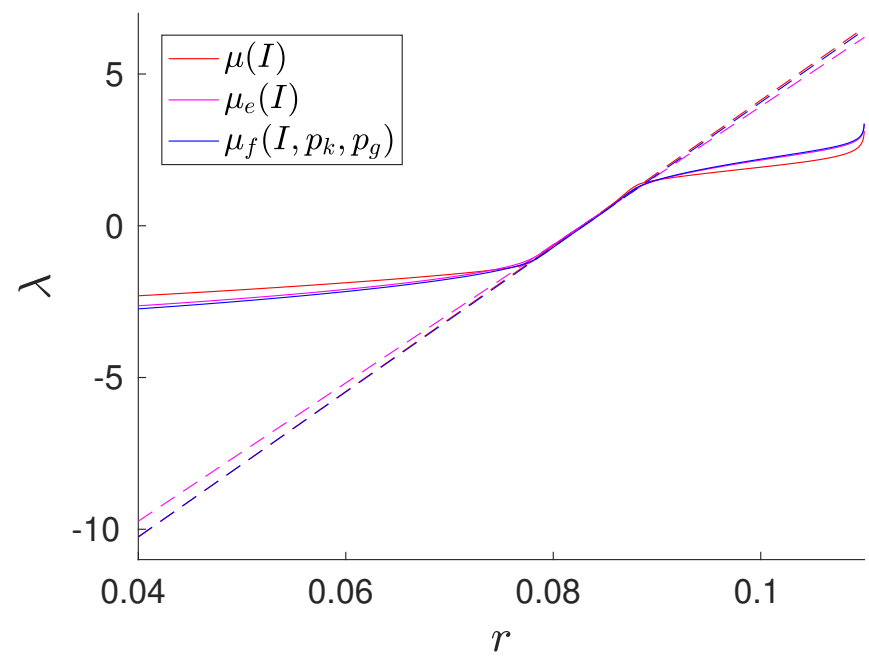

Figure 6.5: Measured coordinate for all rheologies at $H / R_{S}=0.24$, computed using Eq. 6.31. Dashed lines indicate the theoretical coordinate for a symmetric error-function. 
A detailed comparison of these rheologies is done by investigating the shear band characteristics $W(z)$ and $R_{c}(z)$ in Fig. 6.6(a) and Fig. 6.6(b), respectively. The change in width of the shear band as a function of height is captured reasonably well by all rheologies. The rheologies of $\mu(I)$ and $\mu_{e}(I)$ are very comparable, but $\mu_{f}\left(I, p_{k}, p_{g}\right)$ seems to perform slightly better. Deviations from the measured curve are most-likely related to the tails of the shear band not being fully captured.

The centre of the shear band is very well captured by $\mu(I)$ and $\mu_{e}(I)$ which are quite comparable. A significant deviation is observed for $\mu_{f}\left(I, p_{k}, p_{g}\right)$ by introducing gravity and stiffness effects. These effects are both investigated separately to determine their influence. The effect of $p_{k}$ shows a downwards deviation in $R_{c} / R_{S}$. While the $\mu(I)-$ rheology is valid for stiff non-cohesive flows, the extension including particle soft particles $p_{k}$ shows only a minor effect. An upward, more profound trend is observed on $R_{c} / R_{S}$ when only $p_{g}$ is considered. This upwards trend has not been observed in experiments and points out different observations between the DPM coefficients and the experiments.

In Fig 6.7 the friction correction functions are given for both $p_{k}$ and $p_{g}$. Note that as the pressure is constant in radial direction these friction corrections only depend on the $z$-direction. The stiffness correction clearly depends linearly on the pressure and only has a minor impact. The gravity correction has great effect on the friction and especially close to the free surface, confirming the observations in Fig. 6.6(b).

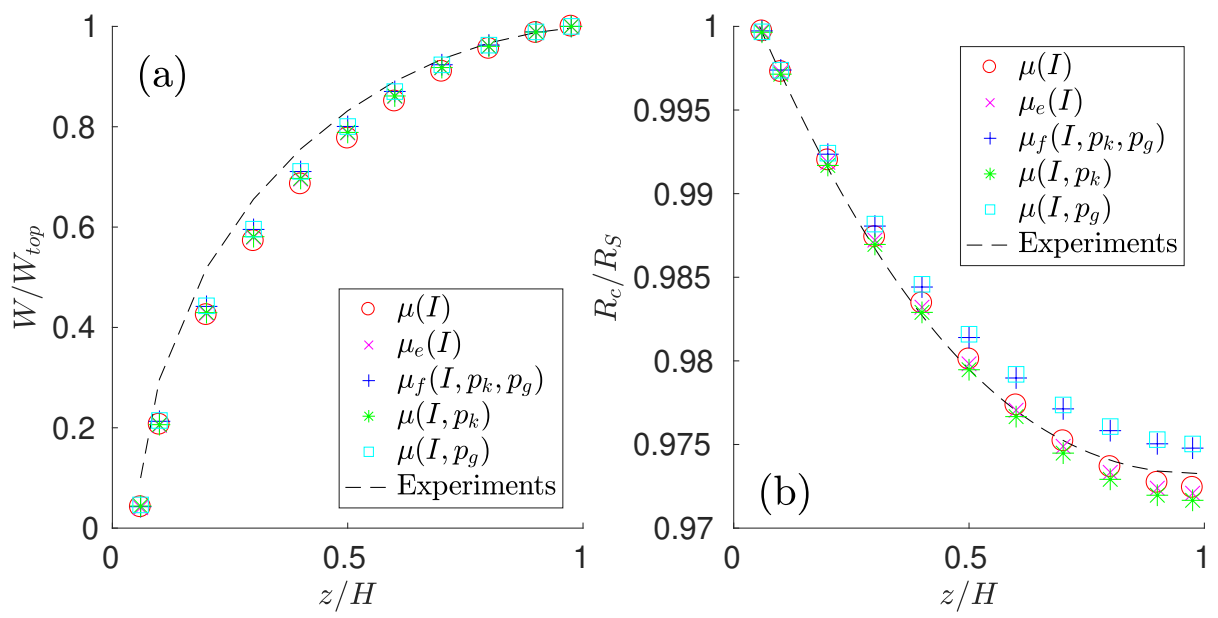

Figure 6.6: Simulations with $H / R_{S}=0.24$ showing (a) Non-dimensional shear band width for a simulation with $H / R_{s}=0.24$. The dashed line is Eq. (6.5) observations from DPM simulations. (b) Non-dimensional shear band centre, with the dashed line the observation from experiments, Eq. (6.3).

Another important characteristic of the flow is the transition between the symmetric regime and asymmetric regime which is governed by the parameter $H / R_{c}$, see section 6.2.1. The behaviour of the different rheologies with respect to $H / R_{c}$ is shown in Fig. 6.8. All rheologies show the transition between symmetric velocity profile at low 


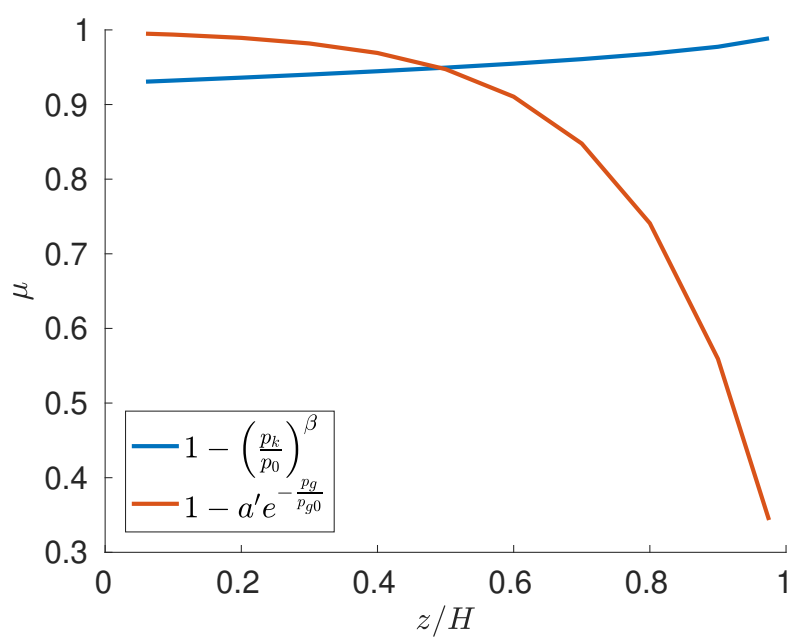

Figure 6.7: Simulations with $H / R_{S}=0.24$ showing the friction correction functions of non-dimensional numbers $p_{k}$ and $p_{g}$ for a simulation of $\mu_{f}\left(I, p_{k}, p_{g}\right)$.

$H / R_{s}$ to the asymmetric regime at high $H / R_{s}$. Note that the simulations use a no-slip boundary condition on the left side, inducing an additional shear band at the boundary for large $H / R_{s}$. Experimental results show that the symmetric regime is valid to $H / R_{S} \approx 0.45$, however all rheologies show asymmetric solutions at this value. A possible explanation could be that the tails are important in this transition, which are not captured accurately by either rheology. This is supported with the observation that for $H / R_{s}=0.45$ a distinct difference is observed between $\mu(I)$ and $\mu(I)_{e}$, while the difference between $\mu(I)_{e}$ and $\mu_{f}\left(I, p_{k}, p_{g}\right)$ is barely observable. The $\mu(I)$-rheology shows, especially around $H=0.45$ a distinct change in behaviour between the shear band and the tail, at approximately $r / R_{o}=0.6$. The other rheologies show a smoother transition. Reason for this is the dependence of the tails on $I$ from Eq. (6.21), while the $\mu(I)$-rheology becomes independent when $\dot{\gamma} \ll \epsilon_{\dot{\gamma}}$.

\subsection{CONCLUSION \& DISCUSSION}

This work presents a solution method for the classical $\mu(I)$-rheology and its generalised descendants. In the former case the implementation is successfully compared with a benchmark [14]. An important difference is the axi-symmetric approach taken in this work in contrast to a Cartesian approach used in the benchmark. Although the axisymmetric approach solves a different set of equations, the solution converged with great accuracy to the benchmark solution.

After this verification of the implementation, the $\mu(I)$-rheology is then investigated together with more advanced rheologies. The two extended rheologies are $\mu_{e}(I)$, see Eq. (6.21), which extends the $\mu(I)$-rheology to very low values of $I$, and a more advanced $\mu_{f}\left(I, p_{k}, p_{g}\right)$-rheology, see Eq. (6.24), which includes effects from particle stiffness $p_{k}$ 

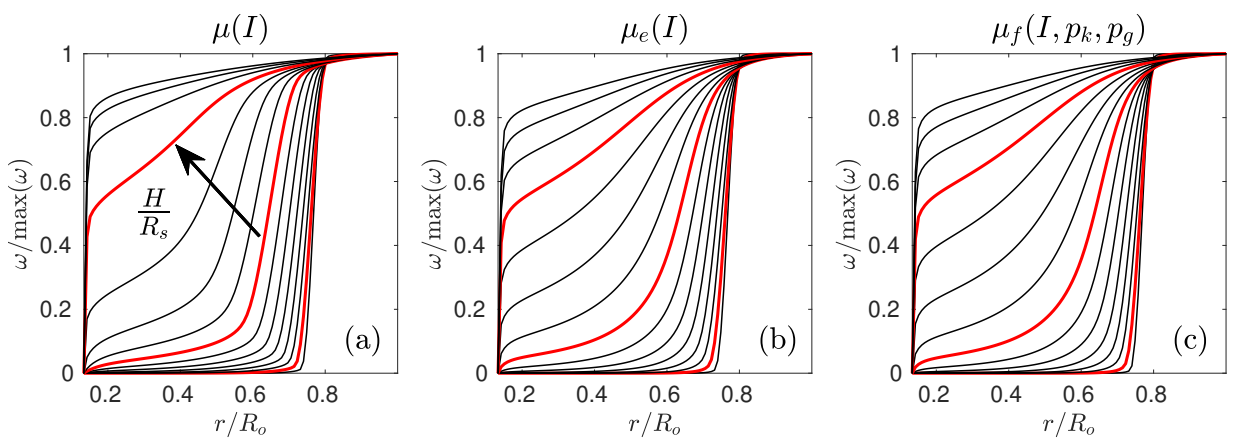

Figure 6.8: Angular velocity of (a) $\mu(I)$, (b) $\mu_{e}(I)$ and (c) $\mu_{f}\left(I, p_{k}, p_{g}\right)$ for changing parameter $H / R_{S}$. Red lines indicate $H=\{0.015,0.045,0.065\}$.

and surface effects $p_{g}$ [15]. A constant density is assumed as simplification, since compressibility effects, see [30], are not yet implemented in the rheologies considered in this work. Alternative rheologies do already include compressibility effects [31-33]. The validation is done in the same setup as was used for the development of the advanced rheologies, a 3D split-bottom ring shear cell, but on its axially-symmetric 2D grid.

Similar to experiments, a shear band is observed in the simulations. All rheologies show good agreement within the shear band, and poor agreement in the tails of the shear band. This includes the extended rheology for low $I$ in $\mu_{e}(I)$, although a small improvement can be observed. The greater added value of $\mu_{e}(I)$ is the removal for arbitrary regularisation, as the best possible regularisation of the $\mu(I)$-rheology used in this work is similar to the extended rheology. In addition, the extended rheology contains extra parameters that have been taken constant in the current work, but could depend on flow parameters such as the granular temperature.

The characteristic shear band width in the bulk non-dimensionaled by the top-width $W / W_{\text {top }}$ is captured well by all rheologies and the extra effect of $p_{k}$ and $p_{g}$ do not have a strong qualitative influence. The non-dimensional shear band center $R_{c} / R_{s}$ for the $\mu(I)$-rheology is comparable to the experimental results for stiff frictional particles and the softness of the particles only moves the centre a bit outwards, in the rather limited range of $p_{k}$ studied here. The extended rheology $\mu_{e}$ is only weakly moving the centre outward. However, the shear band center is influenced significantly by the free surface effect. This effect is quantified by $p_{g}$, the non-dimensional distance from the bulk to the surface. The shear resistance drops from the bulk to the surface, within a bounding layer, by $50 \%$. A curious observation, as this has not yet been observed in experiments which could be due to the low friction $(\mu \approx 0.01)$ of the particles in the DPM simulations. Future investigations could focus on the effect of friction on the shear band center in simulations and the experimental validation of this phenomenon.

Experiments show a symmetric flow profile characterised by an error-function for low filling heights and an asymmetric flow for larger filling heights. This transition is also observed for all rheologies considered here. However, the transition between the 
two regimes is happening at lower fill heights in the simulations. The observations in Fig. 6.8 show a difference in transitional behaviour between $\mu(I)$ and $\mu_{e}(I)$, where the low $I$ extension of $\mu_{e}(I)$ influences the behaviour in the tails. In contrast, the difference between $\mu_{e}(I)$ and $\mu_{f}\left(I, p_{k}, p_{g}\right)$ is barely observable, suggesting that the rheology of the tails play a crucial role in this transition.

To proceed with a comparison between simulations of rheologies, particle scale DPM and experiments, the shear band tails need to be understood better such that the correct transition between symmetric and asymmetric flow is obtained. Besides this, the present rheologies are missing two important ingredients. Granular temperature has been suggested to play a key role in low $I$ flows $[16,34,35]$. Another possible reason for qualitative and quantitative differences between simulations, DPM and experiments is the compressibility effect of the bulk density $\rho[25,30]$. The compressibility effect seems to be small $(\Delta \rho<1-2 \%)$, but might have an enormous effect on the overall rheology. In particular, at small confining stresses (as studied here) the relative distance to jamming is the relevant control parameter $[4,36]$.

\section{REFERENCES}

[1] P. A. Cundall and O. D. L. Strack, A discrete numerical model for granular assemblies, Géotechnique 29, 47 (1979).

[2] J. F. Peters, M. Muthuswamy, J. Wibowo, and A. Tordesillas, Characterization offorce chains in granular material, Physical Review E 72, 041307 (2005).

[3] M. M. H. D. Arntz, W. K. den Otter, W. J. Briels, P. J. T. Bussmann, H. H. Beeftink, and R. M. Boom, Granular mixing and segregation in a horizontal rotating drum: $a$ simulation study on the impact of rotational speed and fill level, AIChE journal 54, 3133 (2008).

[4] N. Kumar and S. Luding, Memory of jamming-multiscale models for soft and granular matter, Granular Matter 18, 58 (2016).

[5] T. Weinhart, A. R. Thornton, S. Luding, and O. Bokhove, From discrete particles to continuum fields near a boundary, Granular Matter 14, 289 (2012).

[6] M. P. van Schrojenstein Lantman and K. Fidkowski, Adjoint-based optimization of flapping kinematics in viscous flows, in 21st AIAA Computational Fluid Dynamics Conference (2013) p. 2848.

[7] Y. Forterre and O. Pouliquen, Flows of dense granular media, Annual Review of Fluid Mechanics 40, 1 (2008).

[8] P. Jop, Y. Forterre, and O. Pouliquen, A constitutive law for dense granular flows, Nature 441, 727 (2006).

[9] P.-Y. Lagrée, L. Staron, and S. Popinet, The granular column collapse as a continuum: validity of a two-dimensional navier-stokes model with a $\mu(I)$-rheology, Journal of Fluid Mechanics 686, 378 (2011). 
[10] J. Chauchat and M. Médale, A three-dimensional numerical model for dense granular flows based on the $\mu(I)$ rheology, Journal of Computational Physics 256, 696 (2014).

[11] G. Daviet and F. Bertails-Descoubes, Nonsmooth simulation of dense granular flows with pressure-dependent yield stress, Journal of Non-Newtonian Fluid Mechanics 234, 15 (2016).

[12] S. Dunatunga and K. Kamrin, Continuum modelling and simulation of granular flows through their many phases, Journal of Fluid Mechanics 779, 483 (2015).

[13] D. G. Schaeffer, Instability in the evolution equations describing incompressible granular flow, Journal of Differential Equations 66, 19 (1987).

[14] S. Mandal, S. Turek, R. Schwarze, M. Haustein, A. Ouazzi, and A. Gladky, Numerical benchmarking of granular flow with shear dependent incompressible flow models, Journal of Non-Newtonian Fluid Mechanics (2018).

[15] S. Roy, S. Luding, and T. Weinhart, A general(ized) local rheology for wet granular materials, New Journal of Physics 19, 043014 (2017).

[16] K. Kamrin and G. Koval, Nonlocal constitutive relation for steady granular flow, Physical Review Letters 108, 178301 (2012).

[17] D. L. Henann and K. Kamrin, A predictive, size-dependent continuum model for dense granular flows, Proceedings of the National Academy of Sciences 110, 6730 (2013).

[18] P. Schall and M. van Hecke, Shear bands in matter with granularity, Annual Review of Fluid Mechanics 42, 67 (2010).

[19] D. Fenistein and M. van Hecke, Kinematics: Wide shear zones in granular bulk flow, Nature 425, 256 (2003).

[20] J. A. Dijksman and M. van Hecke, Granular flows in split-bottom geometries, Soft Matter 6, 2901 (2010).

[21] A. Ries, D. E. Wolf, and T. Unger, Shear zones in granular media: Three-dimensional contact dynamics simulation, Physical Review E 76, 051301 (2007).

[22] G. MiDi, On dense granular flows, The European Physical Journal E 14, 341 (2004).

[23] T. Barker, D. G. Schaeffer, P. Bohorquez, and J. M. N. T. Gray, Well-posed and illposed behaviour of the $\mu(I)$-rheology for granular flow, Journal of Fluid Mechanics 779, 794-818 (2015).

[24] S. Luding, Constitutive relations for the shear band evolution in granular matter under large strain, Particuology 6, 501 (2008).

[25] T. Barker and J. M. N. T. Gray, Partial regularisation of the incompressible $\mu(I)-$ rheology for granular flow, Journal of Fluid Mechanics 828, 5-32 (2017). 
[26] S. Patankar, Numerical Heat Transfer and Fluid Flow, Electro Skills Series (Hemisphere Publishing Corporation, 1980).

[27] M. Heil and A. L. Hazel, oomph-lib-an object-oriented multi-physics finite-element library, in Fluid-Structure Interaction, edited by H.-J. Bungartz and M. Schäfer (Springer Berlin Heidelberg, Berlin, Heidelberg, 2006) pp. 19-49.

[28] C. Taylor and P. Hood, A numerical solution of the navier-stokes equations using the finite element technique, Computers \& Fluids 1, 73 (1973).

[29] S. Mandal, A. Ouazzi, and S. Turek, Modified newton solver for yield stress fluids, in Numerical Mathematics and Advanced Applications ENUMATH 2015, edited by B. Karasözen, M. Manguoğlu, M. Tezer-Sezgin, S. Göktepe, and Ö. Uğur (Springer International Publishing, Cham, 2016) pp. $481-490$.

[30] H. Shi, S. Luding, and V. Magnanimo, Steady state rheology from homogeneous and locally averaged simple shear simulations, in EPJ Web of Conferences, Vol. 140 (EDP Sciences, 2017) p. 03070.

[31] T. Barker, D. Schaeffer, M. Shearer, and J. Gray, Well-posed continuum equations for granular flow with compressibility and $\mu$ (I)-rheology, Proceedings of the Royal Society A: Mathematical, Physical and Engineering Sciences 473, 20160846 (2017).

[32] J. Heyman, R. Delannay, H. Tabuteau, and A. Valance, Compressibility regularizes the $\mu(I)$-rheology for dense granular flows, Journal of Fluid Mechanics 830, 553 (2017).

[33] J. D. Goddard and J. Lee, Regularization by compressibility of the $\mu(I)$ model of dense granular flow, Physics of Fluids 30, 073302 (2018).

[34] P. Kharel and P. Rognon, Shear-induced diffusion in non-local granular flows, EPL (Europhysics Letters) 124, 24002 (2018).

[35] Y. Jiang and M. Liu, Granular solid hydrodynamics, Granular Matter 11, 139 (2009).

[36] N. Kumar, S. Luding, and V. Magnanimo, Macroscopic model with anisotropy based on micro-macro information, Acta Mechanica 225, 2319 (2014). 



\section{7}

\section{CONCLUSIONS AND OUTLOOK}

In this thesis fundamental mechanisms of segregation in dense granular flows have been investigated. In particular, the focus was on a large intruder particle in a monodisperse flow. The research objective to describe the intruder behaviour with a hydrodynamic force model has lead to new fundamental insights in the mechanisms of segregation. The research questions related to this objective, as posed in the introduction, are briefly discussed and concluded before the outlook and recommendations are given.

In Chapter 2 a novel approach was taken to study the direct analogy between an intruder in a dense granular fluid and a particle in a classical Newtonian fluid. A Newtonian flow is considered which is comparable to the granular flow (similar Reynolds number). A particle in such a Newtonian flow experiences a Saffman lift force: a lift force which is directly proportional to a lag in the particle velocity in the flow-direction. To test the direct analogy the following question was posed:

- Q1 Does the intruder experience a lag velocity in flow-direction and is it related to a lift force on the intruder?

By performing DPM simulations in a chute flow setup, it was shown that an intruder does indeed lag in the flow-direction. The implication of this observation is that segregation is not simply a 1D problem, as often posed. The observation of a velocity lag reveals a newly discovered lag force in the stream-wise direction; the origin of this lag force is discussed in Q4. The relation between the measured lift force and the velocity lag was shown to be similar to the Saffman lift force in a chute flow, although it could not be (thus far) validated for other types of dense granular flow configurations.

To describe the behaviour of the intruder with a hydromechanical force model, a good definition of the buoyancy force is required. The classical buoyancy force on an intruder in a fluid is proportional to the fluid volume displaced by the intruder, however for an intruder it is not clear how to define the displaced volume due to the void spaces between particles. The research question in Chapter 3 therefore is:

- Q2 What is the buoyancy force on an intruder particle? 
Two buoyancy force definitions have been discussed: (i) a Voronoi volume approach and (ii) a micromechanical approach. In the Voronoi volume approach it is assumed that the displaced fluid of the intruder is described by its Voronoi volume. The micromechanical approach has shown that the average number of bulk particle contacts per intruder surface area reduces, as the intruder increases in size. Effectively, this mechanism reduces the buoyancy force on the intruder. The Voronoi volume-based correction captures this mechanism well. A wider implication of this structural mechanism is that all other model forces such as lift and drag forces also require a size ratio-dependent correction.

To obtain a more detailed understanding of the segregation lift force a visual approach was taken:

- Q3 What is the effect of the intruder size, density and friction on the granular flow?

High fidelity continuum density, velocity and stress fields have been obtained by coarse graining the discrete particle data. By investigating the density and velocity fields it was shown that the shear flow around the intruder introduces compressional and tensional directions. The flow remains attached to the intruder in the compressional direction while in the tensional direction flow separation occurs. Furthermore, layer formations of flowing bulk particles in the flow-direction are observed.

The origin of segregation has been visualised through observing both the velocity and stress profiles simultaneously; a larger intruder does not fit within a layer of bulk particles and hence the bulk particles of neighbouring layers have to move around the intruder, introducing an anisotropic stress increase in the compressional direction. This increase in stress happens in an asymmetric manner, however it could not be directly understood where the asymmetry came from. The investigated range of density only shows limited influence on this process, while friction significantly changes the velocity and stress fields and affects the lift force on the intruder.

Capturing the lager intruder behaviour in a force model is the ultimate goal of this thesis and hence:

- Q4 Can the segregation force on an intruder be captured by a force model?

In Chapter 4 the lift force mechanism has been observed, but the asymmetry in the anisotropic stress could not be directly explained. Therefore a systematic study was performed in many different flow configurations, focusing on flow gradients which could explain this asymmetry. The gradient in viscosity was the only gradient that correctly determined the segregation direction in all configurations. To test whether the lift forces scale with this gradient, a 2D sheared granular flow configuration with many different boundary conditions was simulated to measure the lift force. Both the lift force perpendicular to the flow as in the newly discovered force in the flow-direction (see Q1) scaled directly with the shear rate and gradient in viscosity, uncovering the origin of segregation. The new lift force scaling also works for a chute flow, implying that the Saffman lift relation found in Chapter 2 could have been a coincidence.

A force model was derived based on the fundamental mechanisms observed in this thesis. The model takes gravity, drag, lift and reference forces into account, where reference forces are a generalisation of the buoyancy force by including forces in the flow- 
direction. This model has the potential to capture reverse segregation for large intruders due to the curvature mechanism discussed in Q2 and Chapter 3.

In order to apply the force model, the shear rate and the viscosity gradient of the undisturbed flow are necessary. It is therefore required to simulate the reference granular flow, preferably with continuum methods for computational efficiency:

- Q5 Can a continuum model using a generalised $\mu(I)$ - rheology simulate granular materials in a split-bottom ring shear cell?

A granular flow solver was developed and validated which can handle a pressure- and shear rate-dependent viscosity. The generalised rheology shows potential for high accurate flow solutions in quasi-static flow regions by incorporating stiffness and surface effects on top of the well-known $\mu(I)$-rheology. For almost-static flow regions it was shown that there is room for improvement, before experimental and DPM comparison can be performed.

\section{OUTLOOK}

This thesis has presented novel insights on segregation in dense granular flows. However, further research is required for a more complete understanding of segregation and for the development of useful tools for industrial problems. A few points are mentioned here as outlook.

\section{- A. Experimental validation}

Developing a segregation model from an experimental setting is very challenging, if not impossible. Instead, it is recommended to validate the DPM-derived lift force experimentally. One possibility is to measure the segregation velocity of a single intruder in a experimental shear box setup [2]. In this experiment different shear rates can easily be obtained and by increasing the (bulk) particle density different viscosity gradients can be obtained.

\section{- B. Lift and drag coefficients}

The size ratio dependence of the lift coefficient is different between sheared flows with vertical and horizontal gravity. The mechanism behind this difference is unknown and requires further investigations. Concerning the drag coefficient, it is not clear whether it depends on the size ratio or if varies with the velocity direction of the intruder. Therefore it is recommended to perform simulations where an intruder is pushed in many different directions for a given granular flow.

\section{- C. Advanced Intruder model}

The force model of the larger intruder derived in this thesis depends only on the size ratio and density ratio, although the range of validity of the density ratio has not been verified. Additionally, it was shown that friction can have a tremendous impact on the lift force. Future investigations could focus on incorporating the friction and density into the drag and lift coefficients. The development of advanced models with more realistic properties such as different shapes will require an extended force model due to the introduction of orientation-dependencies. 
Moreover, angular velocities might also become important in such a model. Another crucial extension of the model is to incorporate flows with a more complicated shear rate tensor. By developing more advanced models it might be possible to engineer granular mixtures with reduced segregation by balancing size, density, friction, cohesion and other realistic particle properties.

\section{- D. Small particle force model}

The current thesis has focused on an extreme situation with only one larger particle in a monodisperse flow. For a more complete understanding of segregation the other extreme, with a single small particle in a monodisperse flow, should also be investigated. This is is a challenging problem as a small particle does not participate in a granular flow like a larger particle. Moreover, obtaining good statistical data on small particles requires serious computational efforts. Whether a fluidbased force model can be obtained for a small particle in a granular fluid remains to be seen.

\section{- E. Segregation models}

Current continuum segregation models could be improved with the newly obtained insights of segregation in the extreme dilute limit. An important step towards a more complete model requires to couple the dilute limit with the 50/50 mixtures. This can be done by investigating the collaboration of multiple intruders.

\section{- F. Segregation prediction tool}

A segregation prediction tool might be developed by combining a continuum granular flow solver together with the intruder force model. When the gradient in viscosity of a granular flow is known, it might be possible to determine the segregated equilibrium state. Whether reverse segregation could be predicted by such tool remains to be seen. The development of such a tool could be useful in industrial engineering for reducing segregation.

\section{- G. New particle simulation method}

A bottleneck of DEM is the incredibly small time step required for accurate and stable simulations. By combining C. and D. it might be possible to predict the direction of any sized particle if the average flow state is known. Such a complete force model might be used in combination with the powerful coarse graining method [1] to develop a new particle simulation method for granular materials.

\section{REFERENCES}

[1] T. Weinhart, A. R. Thornton, S. Luding, and O. Bokhove, From discrete particles to continuum fields near a boundary, Granular Matter 14, 289 (2012).

[2] K. van der Vaart, P. Gajjar, G. Epely-Chauvin, N. Andreini, J. M. N. T. Gray, and C. Ancey, Underlying asymmetry within particle size segregation, Physical Review Letters 114, 238001 (2015). 


\section{A}

\section{A Granular SAFFMAn EFFEct: SUPPLEMENTARY MATERIAL}

\section{A.1. HoRizontAl FORCE BALANCE AND VELOCITY LAG}

Here we introduce a scaling for the lag velocity $\lambda_{x}$ based on the horizontal force balance. Note that by our definition the lag velocity is negative, i.e., the intruder is moving slower than the bulk material at its height. The aim is to show what parameter dependencies are present in the fitting parameter $a$ in Eq. (2.2), which we show here again for convenience:

$$
\lambda_{x}=a(1 / S-1) / \eta
$$

where $\eta=\mu p / \dot{\gamma}$ is the granular viscosity, with $\mu$ the bulk friction, $p$ the pressure, and $\dot{\gamma}=\partial_{z} u_{x}$ the shear rate.

When the size ratio equals one $(S=1)$ we have the following horizontal force balance on the intruder:

$$
F_{r}(S)+F_{g_{x}}(S)=0,
$$

where $F_{g_{x}}=\rho_{p} g_{x} V_{p}$ is the horizontal component of the gravitational force (with $g_{x}=$ $\sin \theta$ ), and $F_{r}$ is the (negative) net horizontal contact force, resembling a "frictional" buoyancy force caused by the shear stress, that cancels gravity.

When the size ratio becomes larger than one $(S>1)$ and the intruder starts to experience a velocity lag $\lambda_{x}$, we propose that the horizontal force balance can be written as:

$$
F_{d}\left(S, \lambda_{x}\right)+F_{r}(S)+F_{g_{x}}(S)=0
$$

where the "frictional" buoyancy force $F_{r}$ become bigger than the downslope gravity force $F_{g_{x}}$, which causes a lag that is damped by $F_{d}$, a Stokesian-like drag working in the same direction as $F_{g_{x}}$ with a dependence on the lag velocity. Note that combined, $F_{d}$ and $F_{r}$ form the contact force, in the $x$-direction, experienced by the intruder,

$$
F_{c_{x}}=F_{d}+F_{r} .
$$



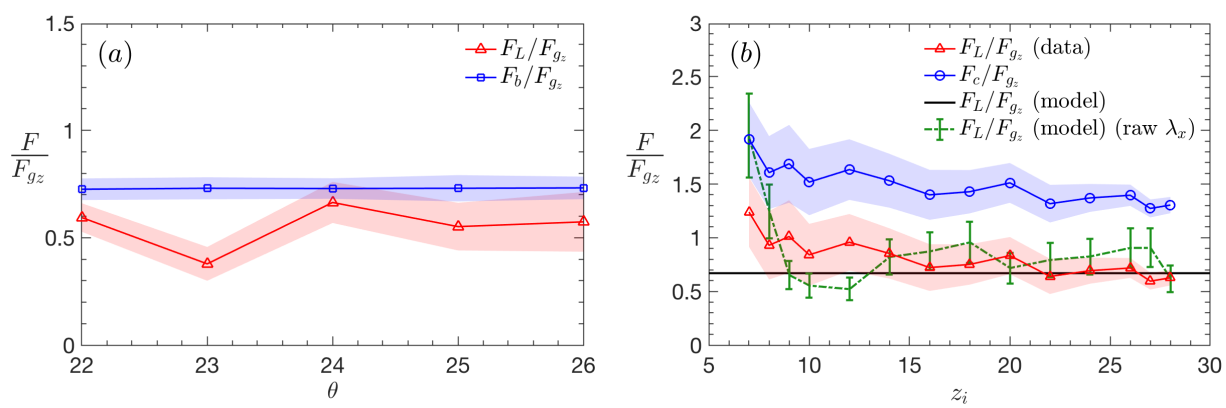

Figure A.1: ( $a$ ) The measured lift force $F_{L}$ (red triangles) and the buoyancy force $F_{b}$ (blue squares) as a function of the chute inclination angle $\theta$, for $S=2.4$. The data are normalised by the vertical component of the gravity force on the intruder $F_{g_{z}}$. (b) The measured net contact force $F_{c}$ (blue circles) and the lift force $F_{L}$ (red triangles) on an intruder, as a function of depth, for $\mathrm{S}=2.4$, normalised by the gravity force. The solid black line is a fit of Eq. (2.9) using the functional form for the lag Eq. (2.2), with $a=0.24$ and $b=130.0$, while the dashed line uses the raw velocity lag data from Fig. 2.2(a). Near the bed $\left(z_{p}<8\right)$ a boundary effect occurs, likely due to layering [2].

In steady state, when the lag is constant in time, there is likely a drag force proportional to the lag velocity, acting in the opposite direction to it, to prevent the intruder from accelerating. The granular Stokes drag introduced by Tripathi and Khakhar [1] is a good candidate for this role:

$$
F_{d}=-c_{d}(S) \pi \eta \lambda_{x} d_{p} .
$$

Here $c_{d}(S)$ is a coefficient, and $d_{p}$ is the diameter of the intruder. Tripathi and Khakhar [1] obtained this drag force for a heavy (higher density) mono-disperse intruder in a chute flow, where they measured the vertical velocity of the sinking intruder. Note that a dependence of $F_{d}$ on $S$ is a possibility, because the drag force appears to be a function of the volume fraction [1] and locally the experienced volume fraction by the intruder changes as function of $S$ (see Fig. 4(a)).

The sum of $F_{r}$ and $F_{g_{x}}$ in Eq. (A.3), which we denote as $\Delta F(S)=F_{r}(S)+F_{g_{x}}(S)$ (analogous to $F_{L}$ in the main-text), can be understood as the upslope directed-in the negative $x$-direction-force causing the intruder to lag. Swapping $F_{d}$ for $-\Delta F(S)$ in Eq. (A.5) and using $d_{p}=S d_{b}$ we obtain an expression for the lag:

$$
\lambda_{x}=\frac{1}{\pi d_{b}} \frac{1}{\eta} \frac{\Delta F(S)}{c_{d}(S) S}
$$

where the first factor is constant, the inverse viscosity represents the second factor, and the $S$-dependence is condensed into the third factor. Hence, the dimensional fitting parameter $a$ in Eq. (2.2) and Eq. (A.1) accounts for the dependency on the bulk particle diameter and unknown dependencies of $c_{d}(S)$ and $\Delta F(S)$.

In order to fully determine $\lambda_{x}$ an assumption needs to be made about the functional form of $\Delta F(S)$. If we would assume that $\Delta F(S)$ is proportional to the shear gradient $\frac{\partial|\boldsymbol{\tau}|}{\partial z}$ and the volume of the particle $V_{p}$ we can write:

$$
\Delta F(S)=f(S) \frac{\partial|\boldsymbol{\tau}|}{\partial z} V_{p},
$$


where $f(S)$ is some $S$-dependent function. This would yield for the lag velocity:

$$
\lambda_{x}=\frac{V_{p}}{\pi d_{b}} \frac{\partial|\boldsymbol{\tau}|}{\partial z} \frac{1}{\eta} \frac{f(S)}{c_{d}(S) S}=v_{b} n(S),
$$

where $v_{b}=\frac{\partial|\boldsymbol{|}|}{\partial z} \frac{d_{p}^{2}}{\eta}$ and $n(S)=\frac{f(S)}{6 c_{d}(S)}$. This would render the $S$-dependent term $n(S)$ dimensionless, while the factor $v_{b}$ is a situation dependent constant with units of velocity, proportional to the viscosity, the gradient in shear stress and the particle diameter. The connection between $v_{b}$ and the constant coefficient $a$ in Eq. (2.2) can be found by writing $v_{b}=a S^{2}$. This reveals that $a \propto \frac{\partial \tau}{\partial z} d_{b}^{2}$. We could proceed in this matter, however, Eq. (A.7) is an assumption that we are not willing to make, so that we use instead Eq. (A.6) in the main text.

\section{A.2. InCLINATION ANGLE DEPENDENCE OF THE LIFT FORCE}

Here we show the dependence of the lift force $F_{L}$, as well as the buoyancy $F_{b}$, on the inclination angle $\theta$ of the chute, for $S=2.4$. These data are plotted in Fig. A.1 $(a)$ where we see that the lift force and the buoyancy force are independent of the inclination angle, within the fluctuations.

\section{A.3. DEPTH DEPENDENCE OF THE LIFT FORCE}

Here we show the dependence of the lift force $F_{L}$, as well as the net contact force $F_{c}=F_{L}+$ $F_{b}$ on the depth of the intruder. These data are plotted in Fig. A.1 $(b)$ where we see that the lift force and the contact force are independent of the depth. This is in agreement with the findings reported by Guillard et al. [3]. There is an increase of both forces close to the bed, but we attribute this to a boundary effect where the intruder particle experiences a greater force due to layering of particles near the bed, as reported by Weinhart et al. [2]. Two fits of the lift force model, Eq. (2.9), are shown in Fig. A.1(b), one using the functional form of the lag $\lambda_{x}=a(1 / S-1) / \eta$ and a second using the raw velocity lag data from Fig. 2.2(b).

\section{REFERENCES}

[1] A. Tripathi and D. V. Khakhar, Numerical simulation of the sedimentation of a sphere in a sheared granular fluid: a granular stokes experiment, Phys. Rev. Lett. 107, 108001 (2011).

[2] T. Weinhart, R. Hartkamp, A. R. Thornton, and S. Luding, Coarse-grained local and objective continuum description of three-dimensional granular flows down an inclined surface, Phys. Fluids 25, 070605 (2013).

[3] F. Guillard, Y. Forterre, and O. Pouliquen, Scaling laws for segregation forces in dense sheared granular flows, J. Fluid Mech. 807 (2016). 



\section{B \\ FORCE MODEL FOR A LARGE INTRUDER: SUPPLEMENTARY MATERial}

\section{B.1. FlOW GRAdientS}

In this appendix the non-dimensional parameter $\alpha$ defined as

$$
\alpha=\frac{\eta \frac{\partial \dot{\gamma}_{x z}}{\partial z}}{\frac{\partial \eta}{\partial z} \dot{\gamma}_{x z}},
$$

is derived for different flow configurations. First some useful definitions and relations are discussed. The flows considered here only have one shear component, $\dot{\gamma}_{x z}$. The shear stress can therefore be expressed as

$$
\tau_{x z}=\eta \dot{\gamma}_{x z},
$$

where $\eta$ is the viscosity. The viscosity is defined as

$$
\eta=\frac{\mu p}{\left|\dot{\gamma}_{x z}\right|},
$$

where $\mu$ is the bulk friction and $p$ is the pressure. Substituting the viscosity definition in Eq. (B.2) gives an alternative expression for the shear stress,

$$
\tau_{x z}=\mu p \operatorname{sgn}\left(\dot{\gamma}_{x z}\right) .
$$

Two useful relations between the the shear gradient and $\alpha$ are

$$
\frac{\frac{\partial \tau_{x z}}{\partial z}}{\frac{\partial \eta}{\partial z} \dot{\gamma}_{x z}}=1+\alpha
$$


and

$$
\frac{\frac{\partial \tau_{x z}}{\partial z}}{\eta \frac{\partial \dot{\gamma}_{x z}}{\partial z}}=\frac{1}{\alpha}+1
$$

The rheology of a dense granular flow is determined by a bulk friction $\mu$ and therefore an assumption is required. Here we assume that $\mu$ is purely a function of the inertial number [1,2],

$$
I=\frac{\left|\dot{\gamma}_{x z}\right| d_{p}}{\sqrt{p / \rho_{p}}}
$$

A useful relation is the derivative of $I$ with respect to $z$,

$$
\frac{\partial I}{\partial z}=\operatorname{sgn}\left(\dot{\gamma}_{x z}\right) \frac{d_{p}}{\sqrt{p / \rho_{p}}} \frac{\partial \dot{\gamma}_{x z}}{\partial z}-\frac{\left|\dot{\gamma}_{x z}\right| d_{p}}{2 \sqrt{p / \rho} p} \frac{\partial p}{\partial z}=\operatorname{sgn}\left(\dot{\gamma}_{x z}\right) \frac{I}{\left|\dot{\gamma}_{x z}\right|} \frac{\partial \dot{\gamma}_{x z}}{\partial z}-\frac{I}{2 p} \frac{\partial p}{\partial z} .
$$

SHEARED FLOW WITH $g_{x}=0$

The gradient of the shear stress vanishes in a sheared system with gravity perpendicular to the flow [3]. Expressing the derivative as

$$
\frac{\partial \tau_{x z}}{\partial z}=\eta \frac{\partial \dot{\gamma}_{x z}}{\partial z}+\frac{\partial \eta}{\partial z} \dot{\gamma}_{x z}=0
$$

and dividing this equation by $\frac{\partial \eta}{\partial z} \dot{\gamma}_{x z}$ gives

$$
\alpha+1=0
$$

yielding $\alpha=-1$. Note that this value is independent of $z$. Therefore, given the value of $\tau_{x z}$, the velocity profile could be analytically derived.

SHEARED FLOW WITH $g_{z}=0$

The gradient of pressure vanishes in a sheared system when gravity is aligned with the flow [3]. Using Eq. (B.4) the gradient of shear stress is

$$
\frac{\partial \tau_{x z}}{\partial z}=\operatorname{sgn}\left(\dot{\gamma}_{x z}\right) \frac{\partial \mu p}{\partial z}=\operatorname{sgn}\left(\dot{\gamma}_{x z}\right) p \frac{\partial \mu}{\partial z} .
$$

The derivative of $\mu$ can be rewritten by performing the chain rule,

$$
\frac{\partial \mu}{\partial z}=\frac{d \mu}{d I} \frac{\partial I}{\partial z}
$$

Substituting Eq. (B.8) and noting that $\frac{\partial p}{\partial z}=0$ yields

$$
\frac{\partial \mu}{\partial z}=\frac{d \mu}{d I} \operatorname{sgn}\left(\dot{\gamma}_{x z}\right) \frac{I}{\left|\dot{\gamma}_{x z}\right|} \frac{\partial \dot{\gamma}_{x z}}{\partial z}
$$

Substituting this result into Eq. (B.11) gives

$$
\frac{\partial \tau_{x z}}{\partial z}=\frac{I p}{\left|\dot{\gamma}_{x z}\right|} \frac{d \mu}{d I} \frac{\partial \dot{\gamma}_{x z}}{\partial z}
$$




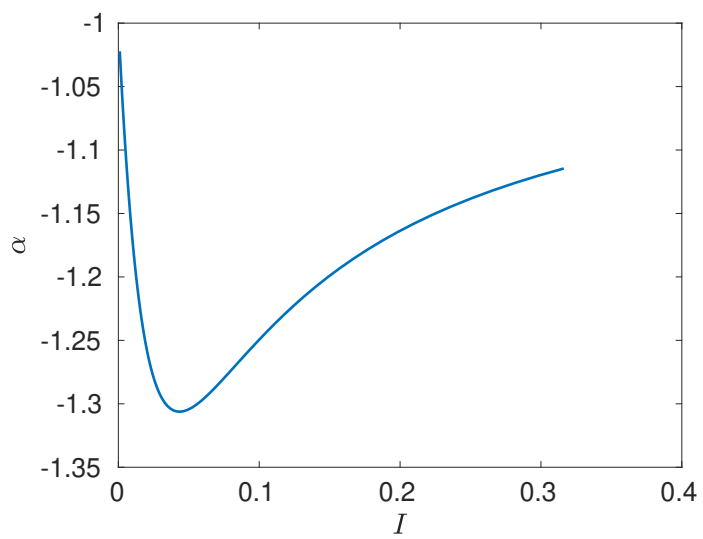

Figure B.1: Estimation of Eq. (B.16) using realistic material coefficients.

Divide both sides by $\eta \frac{\partial \dot{\gamma}_{x z}}{\partial z}$ and using Eq. (B.6) gives

$$
1+\frac{1}{\alpha}=\frac{I}{\mu} \frac{d \mu}{d I}
$$

Therefore,

$$
\alpha=\frac{1}{\frac{I}{\mu} \frac{d \mu}{d I}-1} .
$$

This is a general result without making further assumptions on the specific shape of $\mu$. In order to estimate the sign of $\alpha$, the $\mu(I)$-rheology is adopted [1, 2],

$$
\mu=\mu_{s}+\frac{\Delta \mu}{I_{0} / I+1}
$$

where $\mu_{s}, \Delta \mu$ and $I_{0}$ are constants. With this definition it can be shown that

$$
\lim _{I \rightarrow 0} \alpha=-1
$$

and

$$
\lim _{I \rightarrow \infty} \alpha=-1 \text {. }
$$

Realistic values for the rheology are $\mu_{s}=0.15, \Delta \mu=0.24$ and $I_{0}=0.07$ [4]. In Fig. B.1 $\alpha$ is shown. From this estimation it is assumed that $\alpha<0$.

\section{Chute Flow}

In a chute flow, the friction is constant [5]. Therefore, using Eq. (B.4), the gradient in shear stress can be expressed as

$$
\frac{\partial \tau_{x z}}{\partial z}=\operatorname{sgn}\left(\dot{\gamma}_{x z}\right) \mu \frac{\partial p}{\partial z}
$$


Dividing by $\eta \frac{\partial \dot{\gamma}_{x z}}{\partial z}$ and substituting Eq. (B.6) yields

$$
1+\frac{1}{\alpha}=\operatorname{sgn}\left(\dot{\gamma}_{x z}\right) \frac{\mu \frac{\partial p}{\partial z}}{\eta \frac{\partial \dot{\gamma}_{x z}}{\partial z}}
$$

The constant friction in a Bagnold profile implies a constant inertial number. Hence,by using Eq. B.8, the shear rate can be expressed as

$$
\frac{\operatorname{sgn}\left(\dot{\gamma}_{x z}\right)}{\left|\dot{\gamma}_{x z}\right|} \frac{\partial \dot{\gamma}_{x z}}{\partial z}=\frac{1}{2 p} \frac{\partial p}{\partial z}
$$

Multiplying both sides with $\operatorname{sgn}\left(\dot{\gamma}_{x z}\right) \mu p$ and using Eq. B.3 yields

$$
\eta \frac{\partial \dot{\gamma}_{x z}}{\partial z}=\operatorname{sgn}\left(\dot{\gamma}_{x z}\right) \frac{\mu}{2} \frac{\partial p}{\partial z}
$$

substituting this in Eq. B.21 yields

$$
1+\frac{1}{\alpha}=\frac{\operatorname{sgn}\left(\dot{\gamma}_{x z}\right) \mu \frac{\partial p}{\partial z}}{\operatorname{sgn}\left(\dot{\gamma}_{x z}\right) \frac{\mu}{2} \frac{\partial p}{\partial z}}=2
$$

and therefore in a Bagnold profile $\alpha=1$. Note that Eq. (B.23) is constant in $z$ and therefore by extension $\dot{\gamma}_{x z} \frac{\partial \eta}{\partial z}$.

\section{SHEAR BOX}

In a shear box the parallel walls enforce a flow with $\frac{\partial \dot{\gamma}_{x z}}{\partial z}=0$ and a presence of gravity in the $z$-direction yields $\frac{\partial p}{\partial z}<0$. Substituting this directly in Eq. (B.1) yields $\alpha=0$. However this does not imply that the gradient of viscosity is absent. The gradient in viscosity can be expressed as

$$
\frac{\partial \eta}{\partial z}=\frac{\partial}{\partial z} \frac{\mu p}{\left|\dot{\gamma}_{x z}\right|}=\frac{\mu}{\left|\dot{\gamma}_{x z}\right|} \frac{\partial p}{\partial z}+\frac{p}{\left|\dot{\gamma}_{x z}\right|} \frac{\partial \mu}{\partial z}
$$

The derivative of the friction can be rewritten by performing the chain rule and inserting Eq. (B.8),

$$
\frac{\partial \mu}{\partial z}=\frac{d \mu}{d I} \operatorname{sgn}\left(\dot{\gamma}_{x z}\right)\left(\frac{I}{\left|\dot{\gamma}_{x z}\right|} \frac{\partial \dot{\gamma}_{x z}}{\partial z}-\frac{I}{2 p} \frac{\partial p}{\partial z}\right)
$$

Substituting this result back into Eq. (B.25) and using $\frac{\partial \dot{\gamma}_{x z}}{\partial z}=0$ yields

$$
\frac{\partial \eta}{\partial z}=\frac{1}{\left|\dot{\gamma}_{x z}\right|}\left(\mu-\frac{I}{2} \frac{d \mu}{d I}\right) \frac{\partial p}{\partial z}=\frac{1}{\left|\dot{\gamma}_{x z}\right|} \beta \frac{\partial p}{\partial z},
$$

with

$$
\beta=\mu-\frac{I}{2} \frac{d \mu}{d I}
$$

To determine the sign of $\beta$, assume the $\mu(I)$-rheology and note that

$$
\frac{d \mu}{d I}=\frac{\Delta \mu I_{0}}{\left(I_{0}+I\right)^{2}}
$$


and

$$
\frac{d^{2} \mu}{d I^{2}}=\frac{-2}{I_{0}+I} \frac{d \mu}{d I} .
$$

The limit of $\beta$ for $I \rightarrow 0$ can then easily be estimated,

$$
\lim _{I \rightarrow 0} \beta=\mu_{s}
$$

The gradient of $\beta$ is

$$
\frac{d \beta}{d I}=\frac{d \mu}{d I}-\frac{1}{2} \frac{d \mu}{d I}-\frac{I}{2} \frac{d^{2} \mu}{d I^{2}} .
$$

Using Eq. B.30 this can be simplified to

$$
\frac{d \beta}{d I}=\left(\frac{1}{2}+\frac{I}{I_{0}+I}\right) \frac{d \mu}{d I} .
$$

Note that by definition $I$ is positive and Eq. (B.29) is also positive. The gradient of $\beta$ is there fore always positive. Combining this with Eq. (B.31) shows that $\beta>0$. Therefore, the gradient in viscosity, Eq. (B.27), is always negative in a shear box.

\section{REFERENCES}

[1] GDR-MiDi, On dense granular flows, Eur. Phys. J. E 14, 341 (2004).

[2] P. Jop, Y. Forterre, and O. Pouliquen, A constitutive law for dense granular flows, Nature 441, 727 (2006).

[3] F. Guillard, Y. Forterre, and O. Pouliquen, Scaling laws for segregation forces in dense sheared granularflows, Journal of Fluid Mechanics 807 (2016), 10.1017/jfm.2016.605.

[4] S. Roy, S. Luding, and T. Weinhart, A general (ized) local rheology for wet granular materials, New journal of physics 19, 043014 (2017).

[5] T. Weinhart, A. R. Thornton, S. Luding, and O. Bokhove, Closure relations for shallow granular flows from particle simulations, Granular Matter 14, 531 (2012). 



\section{$\mathbf{C}$}

\section{Parallel Particle Simulations IN MERCURYDPM}

This work presents a parallel computing algorithm for the discrete particle method (DPM) simulations using MPI, implemented in the open-source software package MercuryDPM. The algorithm is described in great detail such that it can be used as guide for future MercuryDPM developers. The algorithm can handle complex boundaries such as periodic boundaries, insertion/deletion boundaries and maser boundaries (a set periodic boundaries which emits particles into a simulation). The weak scaling of the algorithm shows a 40\% reduction for the first 60 cores used. However, for simulations with more cores the efficiency stays constant. Future recommendations are to implement automatic load balancing and efficient data generation by employing coarse grained continuum fields.

\section{C.1. INTRODUCTION}

Many industrial sectors require handling of granular materials such as the food, pharmaceutical and mining industry. Designing efficient equipment is a primary target for the industry to reduce energy consumption and operation costs. The quest for optimal designed equipment is hampered due to a lack of understanding of granular materials. Computer simulations such as particle simulations can aid in a cheaper and more flexible design processes.

A drawback of particle simulations is the shear amount of time required to simulate industrial scale problems. There are many possibilities to reduce the simulation time. One approach is by up-scaling the physical model, such as the use of meso-scale particles [1] or continuum models [2]. A multi-scale approach combines the particle simulation methods and up-scale methods and applies the appropriate method where necessary [3]. Alternatively, the simulation time of particle simulations can be reduced by means of parallel computation. In this appendix the latter method is designed and implemented in the open source software package MercuryDPM [4].

Simulations have been performed by W.M. den Breeijen. 
MercuryDPM uses the discrete particle method (DPM) [5] to simulate particles. An advanced hierarchical grid is used to efficiently deal with polydisperse systems [6, 7]. It can handle complex geometries including axially symmetric, polyhedral and helical screw walls. Furthermore MercuryDPM contains specialised boundaries such as a mass laser (maser) boundary [8] and angled periodic boundaries [9]. The implementation of a parallel algorithm in MercuryDPM requires compatibility with these features.

An overview of parallel methods is given in section C.1.1 and an overview of the DPM algorithm used by MercuryDPM is given in section C.1.2. In section C. 2 the implementation structure and details are discussed. Scaling results are shown in C.3 and conclusions and discussions are given in section C.4.

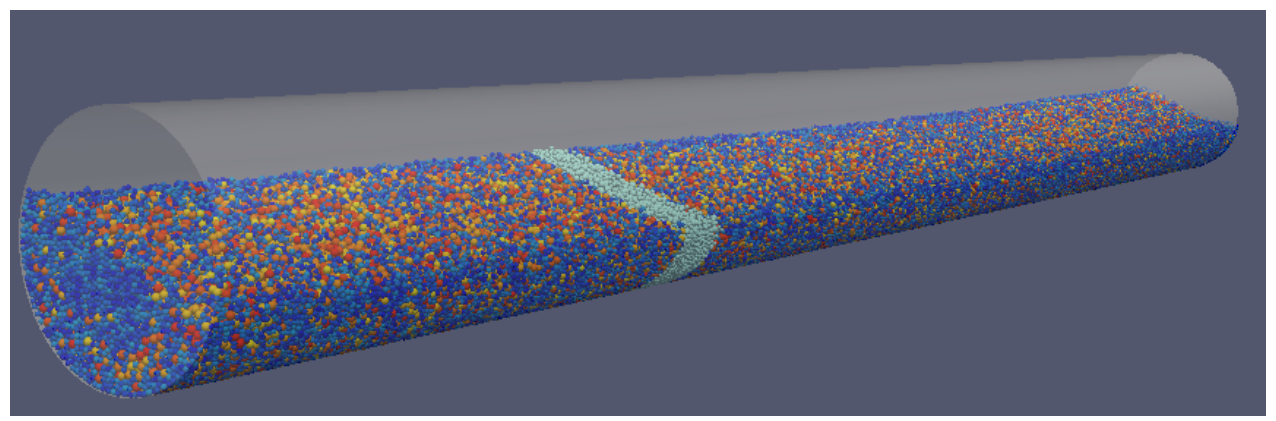

Figure C.1: A parallel simulation of a rotating drum in MercuryDPM using 36 cores. The drum contains a bidispersed mixture of large (red) and small (blue) particles with some degree of polydispersiy. The light blue particles are the particles on a single core.

\section{C.1.1. Parallel methods}

Parallel methods can be applied to algorithms which can be split into smaller simultaneously executed processes. There are generally two parallelisation strategies: shared memory and distributed memory. Shared memory implies that the simulation is performed over many different cores or threads, but the memory is accessible to all the processes. Distributed memory means that processes don't have access to all memory and therefore have to communicate information.

For relative small particle simulations $\left(\sim 1.0 \times 10^{6}\right.$ particles $)$ the shared memory approach is a common choice. Most DPM software have implemented this type of parallisation of particle methods [10-13]. The shared memory approach is generally performed on a Graphics Processing Unit (GPU), due to the large amount of cores on these cards and their fast memory. A limiting factor of this approach is the finite amount of memory and cores on a single GPU. State of the art GPU's have a computational power of up to 100 Central Processing Units (CPU) [14]. For more demanding simulations a distributed memory approach is required.

When applying the distributed memory approach to a DPM algorithm, not all simulation information is readily available for each process. Therefore the simulation is geometrically divided into a grid of domains. These domains will perform the simulation locally and require additional simulation information at the domain boundaries by 
communicating with their neighbour domains. The standard communication protocol for the distributed approach is called message passing interface (MPI) [15]. This interface allows to implement a communication strategy which is independent of the computer architecture. The actually implementation of the MPI protocol depends on the computer architecture and therefore offers various choices. Examples of implementations are openMPI [16] and MPICH [17]. As the interface definitions of the MPI protocol do not change, the code is very maintainable. The distributed approach is less common for DPM software packages as only a handful have take this approach [18].

An alternative approach is a hybrid distributed/shared approach, where locally a domain applies the shared memory approach. This approach has also been implemented and investigated in [18]. Here MPI is used as general domain decomposition and load balancing is done by local threads. The results show that for low number of cores the distributed approach outperforms the shared memory and hybrid approach. However, as the number of cores increase the hybrid approach becomes more feasible as load balancing is improved in the hybrid approach. Implementing a full hybrid approach with load balancing is an interesting approach for MercuryDPM. As a first step an MPI approach is implemented, because this removes the hardware constraints of a shared memory approach.

The design of the parallel code can be split up into two distinct problems which are different in communication style. The first problem consists of decomposing the simulation in smaller sub domains. Each domain communicates only with its neighbouring domains. This communication is called local communication as the domains only communicate locally. The second communication problem is termed global communication where a-priori it is unknown which domains have to communicate. Examples of global communication are periodic boundaries and insertion boundaries. The local communication structure is discussed in section C.2.1 and the global communication structure in section C.2.2.

\section{C.1.2. MERCURYDPM SIMULATION METHOD}

The implementation of the DPM algorithm greatly influences the implementation of a parallel code and is therefore explained in detail in this section.

The trajectory of each particle is solved by employing Newton's equation of motion, $\boldsymbol{F}=m \boldsymbol{a}$, where $\boldsymbol{F}$ are the forces on the particle, $m$ is the mass of the particle and $\boldsymbol{a}$ the acceleration. The angular motion is solved in a similar manner, $\boldsymbol{T}=\boldsymbol{I} \cdot \boldsymbol{\alpha}$, with torque $\boldsymbol{T}$, moment of inertia $\boldsymbol{I}$ and the angular acceleration $\boldsymbol{\alpha}$. The forces on a particle are caused by contacts with other particles, body forces such as the gravity force, or long range forces.

The numerical scheme that approximates the equations of motion in MercuryDPM is the velocity Verlet scheme [19]. This scheme consists of a three step process. Note that both the translational and rotational motions are solved using this scheme, but the explanation is given by only considering the translational properties. The first part computes the half-time step velocity and the next time step position,

$$
\boldsymbol{v}^{n+\frac{1}{2}}=\boldsymbol{v}^{n}+\frac{1}{2} \mathbf{a}^{n} \Delta t,
$$




$$
\boldsymbol{x}^{n+1}=\boldsymbol{x}^{n}+\boldsymbol{v}^{n+\frac{1}{2}} \Delta t,
$$

where $\boldsymbol{x}, \boldsymbol{v}, \boldsymbol{a}$ indicate, respectively, position, velocity and acceleration of the particle and $n$ indicates the current time step. The second part of velocity Verlet computes the new acceleration on the particles,

$$
\boldsymbol{a}^{n+1}=\boldsymbol{F}_{p}^{n+1}\left(\boldsymbol{x}^{n+1}, \boldsymbol{v}^{n+\frac{1}{2}}\right) / m_{p}
$$

with the total force on the particle at the new time step $\boldsymbol{F}_{p}^{n+1}$ and the mass of the particle $m_{p}$. During this step interactions between particles are evaluated and the resulting contact forces are computed based on a specified contact law. Examples of commonly used contact laws are the linear viscoelastic contact law [20], and the Hertz-Mindlin contact law [21]. These laws require the (angular) position and (angular) velocity of the interacting particles, and history parameters of the interaction. Note that at this point of the simulation all particles require the most recent information of neighbouring particles, implying communication between processes in case of a distributed parallel approach. All real particle have obtained new positions in the first step, that implies that new particles might have to be communicated to neighbour domains.

Finally, in the third step the velocity at the time step is computed

$$
\boldsymbol{v}^{n+1}=\boldsymbol{v}^{n+\frac{1}{2}}+\frac{1}{2} \mathbf{a}^{n+1} \Delta t
$$

The considered particle does not depend on neighbouring particles to perform this update, so no communication is required.

\section{C.2. Parallel Algorithm}

In this section the parallel algorithm is explained. First the local communication is explained which deals with neighbouring domains communication with each other. sections C.2.1 and C.2.2 introduce concepts and definitions of the algorithm and the detailed implementation into MercuryDPM is elaborated in section C.2.4.

\section{C.2.1. LOCAL COMMUNICATION STRUCTURE}

Each particle simulation in MercuryDPM has a simulation box size which specifies the approximate size of the simulation. This simulation box is not enforced on the particles, enabling the possibility of particles traveling outside the simulation box. This is particularly useful for 1D-decompositions with free surfaces. Based on this simulation box the simulation is subdivided into a Cartesian grid of $n_{x} \times n_{y} \times n_{z}$ domains. An example of a 2D hourglass simulation is sketched in Fig C.2. In this specific example the total amount of domains is 9. Each domain is linked to a process and particles located inside the domain belong to that specific domain and are handled by that domain only. The domain edges of domains that are located at the boundary of the grid extend into infinity. The set of all domains is $D$ and $D_{n}\left(D_{i}\right) \subseteq D$ are all domains that are neighbours of domain $D_{i}$. Particles that are located precisely on a domain boundary always belong to the domain on the right side in their respective axis.

Particles located close to the boundary of a neighbouring domain can have interactions with particles located in a neighbouring domain. To compute the contact force 


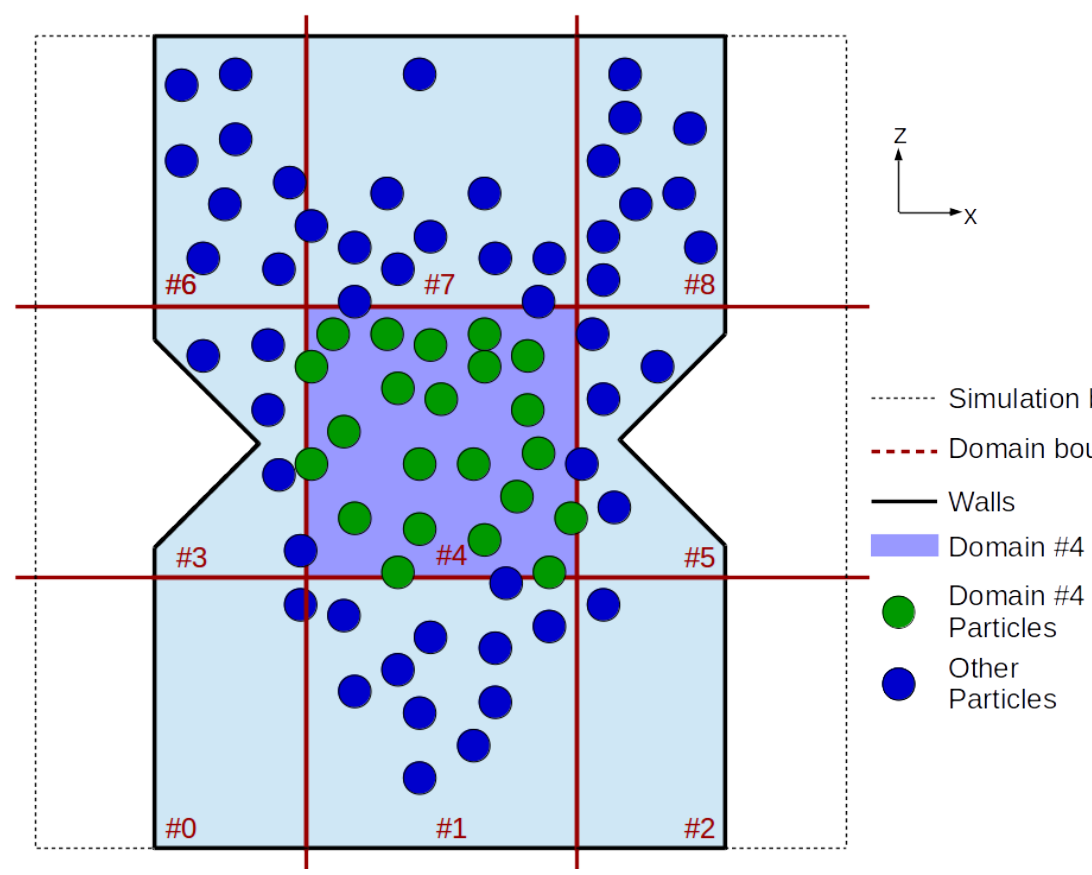

Figure C.2: Decomposition of a 2D hourglass simulation using a Cartesian grid of $3 \times 1 \times 3$, with a total of 9 cores. The decomposition is based on the simulation box which is given as user input in MercuryDPM, as are the number of cores in each Cartesian direction. Domain edges on the boundary of the simulation box extend towards infinity. All particles located inside a domain are only known on that particular domain, generating 9 parallel simulations. 
with these particles, see Eq. (C.3), ghost particles are introduced. The domain is extended into the other domains and ghost particles are introduced in this extended region. A sketch of two domains communicating which each other is shown in Fig C.3. The size of the overlapping region is called the interaction distance $I$ which is always two times the largest interaction radius. This can be observed by considering the largest possible constant distance between particles if one of them is located on a domain boundary. Generally the interaction distance is twice the largest particle radius, but in case of long range forces it becomes twice the longest interaction radius.

Introducing a communication domain also introduces a CFL-like condition [22]. The speed at which a particle travels through the domain should be smaller than the overlapping zone. This poses the following constraint on the particle velocity,

$$
\max \left(\left|v_{p}\right|\right) \Delta t<\frac{1}{2} I
$$

where $\left|v_{p}\right|$ is the maximum absolute velocity of a particle in the domain and $\Delta t$ is the time step of the simulation. Generally this does not pose problems for simulations, because if these kind of velocities are obtained, the simulation can't even simulate particleparticle contacts correctly and hence this criteria will always be satisfied in a sensible simulation.

The communication between domains introduce various different particle types. For the local communication between neighbouring domains the particles are distinguished into three types. An $R$-particle is a real particle located on domain $D_{i}$ and is far away from any domain boundary. If an $R$-particle moves within a distance of $I$ of a domain boundary it is flagged as an $M$-particle. This implies the particle needs to communicate its properties to the neighbouring domain. The neighbouring domain $D_{j}$ of $D_{i}$ has a copy of this $M$-particle, but has no control over it and hence it is called the ghost of the $M$-particle, or in short $M G$-particle. The $M G$-particles receive their particle properties from the corresponding $M$-particles. A list of particle types is used in the communication is given in Table C.1.

Two approaches can be taken with regard to constructing $M G$-particles. The first approach creates $M G$-particles every time step and after the forces between $M$-particles and $M G$-particles have been computed, the $M G$-particles are destroyed. The second approach does not delete the $M G$-particle, but instead updates the position and velocity every time step. Based on the new position the status of the particle can be changed to an $M$-particle or it can be deleted. The downside of the second approach is that a positional check is required to see if the type of a particle has changed. The downside of the first approach is an increased amount of data that has to be communicated using MPI routines, including history parameters of interactions between particles. The number of parameters that have to be communicated in the first approach can increase dramatically when considering spherical particles [23] or complex interaction laws such as Hertz-Mindlin [21]. The second strategy is therefore chosen for MercuryDPM.

Keeping track of all $M$ - and $M G$-particles requires efficient bookkeeping. The example in Fig. C.3 only considers two neighbouring domains such that each $M$-particle corresponds to one $M G$-particle, however in a Cartesian mesh an $M$-particle located in a corner of a domain has to be copied to multiple neighbouring domains. To describe 

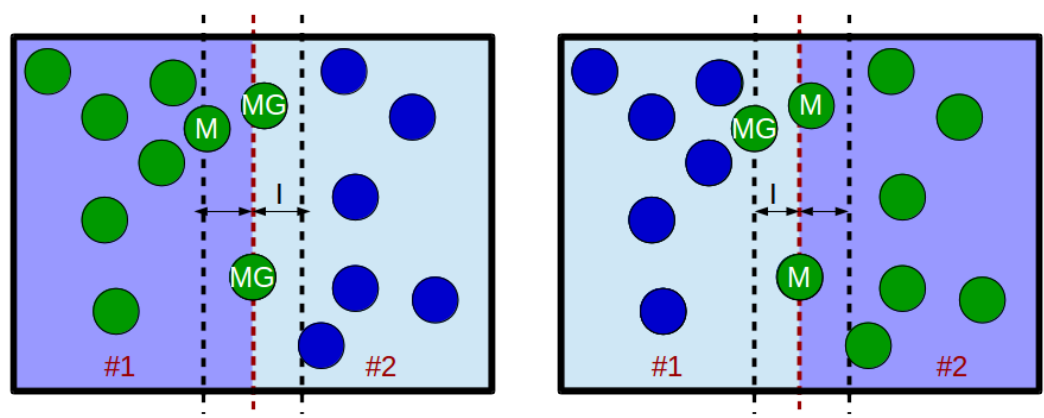

Figure C.3: Sketch of communications between domains. Active particles on a domain are $R$-particles and become $M$-particles if their distance towards the domain boundary is smaller than the interaction distance $I$. The neighbouring domain contains ghost particles ( $M G$-particles) of these $M$-particles.

this process a communication index $\mathscr{C}$ is introduced. This number indicates how the particle is interacting with neighbour domains

$$
\mathscr{C}=i+3 * j+9 * k+13
$$

where $\{i, j, k\}$ is the local coordinate see Fig C. 4 for a 2D sketch. This uniquely defines the possible interactions of the particle with neighbour domains. If this number changes it implies that the interaction with neighbour domains also changes. To indicate with how many domains a particle is interacting with, an absolute communication complexity is defined,

$$
|\mathscr{C}|=|i|+|j|+|k|
$$

In the $2 \mathrm{D}$ mesh of Fig C.4 the maximum value of $|\mathscr{C}|$ is 2 , which corresponds to a particle that has ghosts on three neighbour domains (2 sides and one rib). In 3D the maximum $|\mathscr{C}|$ is 3 . The corresponding neighbours are 3 sides, 3 ribs and one corner, a total of 7 neighbours.

Each domain $D_{i}$ contains lists that keep track of $M$-particles and $M G$-particles on its own domain. The boundary particle list $\mathscr{B}_{i j}$ keeps track of the $M$-particles on domain $D_{i}$ that have a corresponding $M G$-particle on domain $D_{j}$. Domain $D_{i}$ also keeps a list of all $M G$-particles in its own domain, $\mathscr{G}_{i j}$, where $D_{j}$ is the domain that has the corresponding $M$-particle. Operations on these lists are designed in such a way that the following statement can be guaranteed,

$$
\mathscr{B}_{i j}=\mathscr{G}_{j i} .
$$

When $M G$-particles are updated with the properties of the corresponding $M$-particles, no particle search step is required, because the position in the receiving data structure is already known. Note that with this implementation it can occur that an $M$-particle with $|\mathscr{C}|=3$ located on domain $D_{i}$ is listed 7 times in $\mathscr{B}$ and the corresponding $M G$-particle is located 7 times in $\mathscr{G}$. 


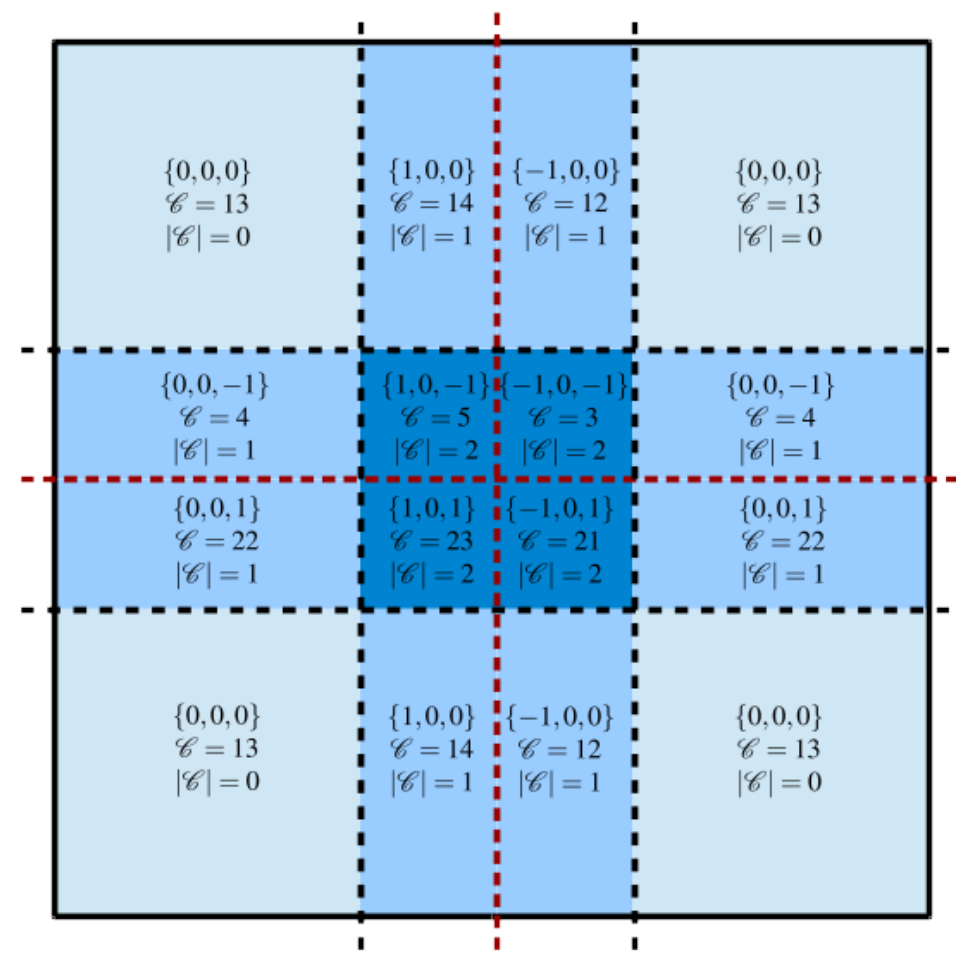

Figure C.4: Each communication zone has a specific local coordinate $\{i, j, k\}$ which can be converted into a unique communication index $\mathscr{C}$. The value $|\mathscr{C}|$ indicates the complexity of interaction. With $|\mathscr{C}|=1$ the communication zone is only interacting with one neighbour domain. In a 2D Cartesian mesh the maximum complexity is two, corresponding to ghost particles on three neighbouring domains.

\begin{tabular}{|l|l|}
\hline Particle type & Definition \\
\hline R-particle & A normal particle away from any parallel communication zones. \\
$M$-particle & An R-particle that is in the MPI communication zone. \\
$M G$-particle & A ghost of an M-particle located on another domain. \\
$P$-particle & An R-particle that is in the periodic communication zone. \\
$P G$-particle & A ghost of a P-particle at the corresponding periodic boundary. \\
$P M$-particle & Particle located in a $P$ and $M$ boundary at the same time \\
$P M G$-particle & An $M G$ ghost particle of a $P M$-particle \\
$P G M^{*}$-particle & A $P G$-particle and an $M$-particle, but not listed in $\mathscr{B}$. \\
$P G M G^{*}$-particle & A $P G$-particle and an $M G$-particle, but not listed in $\mathscr{G}$. \\
\hline
\end{tabular}

Table C.1: Definition of types of communicating particles. 
The local communication structure now fully describes the communication between domains. Ghost particles are introduced in a communication zone of $I$ from the boundary. Bookkeeping lists $\mathscr{B}$ and $\mathscr{G}$ keep track of communicating particles. Based on the particle type and complexity coordinate $\mathscr{C}$ a particle can be assigned to the correct lists and due to the symmetry between $\mathscr{B}$ and $\mathscr{G}$, see Eq. (C.8), quick communication and processing of information is possible. The detailed implementation of the algorithm is given in section C.2.4, however first the structure for global communication needs to be introduced as these two structures affect each other.

\section{C.2.2. PERIOdiC BOUNDARY COMMUNiCATION STRUCTURE}

Periodic boundaries are a set of boundaries that are used to teleport particles from one boundary to the other boundary when crossed. There are various different types of periodic boundaries used in DEM simulations. One example is the maser boundary, see Fig. C.5 [8]. Other examples are angled periodic boundaries used in shear cells [9] and compaction cubes [].

Although the idea of periodic boundaries is very similar in these examples, they differ in a subtle way. Angled periodic boundaries require not only a positional change of the particle, but also a switch in velocity direction. In most flows all particles are within the periodic boundaries which is not the case with the maser. In case of a compaction cube, the periodic boundaries are compressed by moving the periodic boundaries. To develop a parallel algorithm that can take all these differences into account it is not feasible to couple periodic boundaries to domain boundaries.

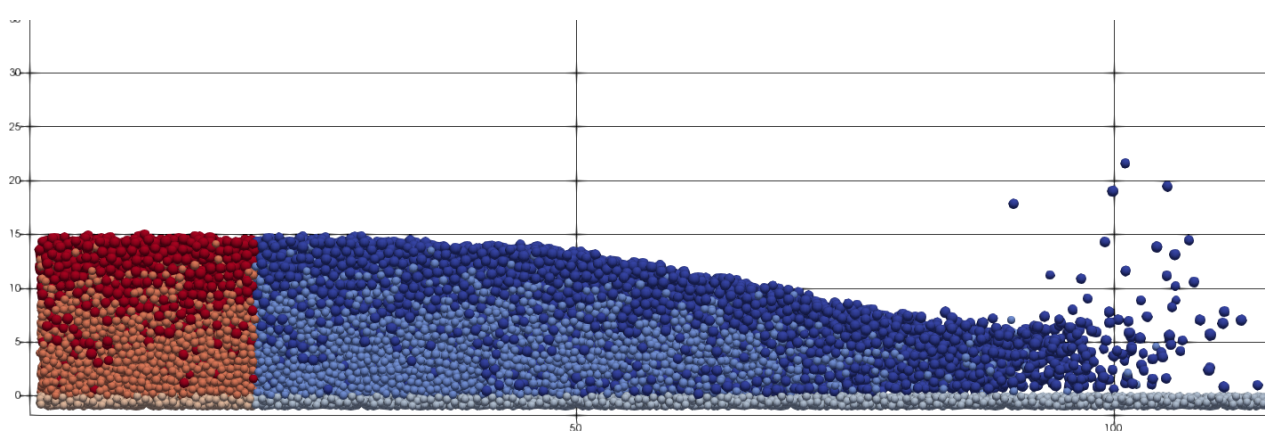

Figure C.5: A maser inflow boundary. Red particles belong to a periodic box. Red particles moving over the right periodic boundary get both copied back to the left boundary and escape the periodic box, creating an outflow with chute flow characteristics. Figure created by I.F.C. Denissen.

Similar to the local communication, communication zones are introduced at periodic boundaries with a similar interaction distance of $I$, twice the largest interaction radius in the simulation. A periodic particle $P$-particle is defined as a real particle that is located in the communication zone of a periodic boundary, see Fig. C.6. Here a $P$-particle is located at the top of a periodic boundary and a $P G$-particle, a ghost of a $P$-particle is therefore generated at the bottom. Note that in this particular case the $P G$-particle resides on the same domain, but this is generally not the case. 

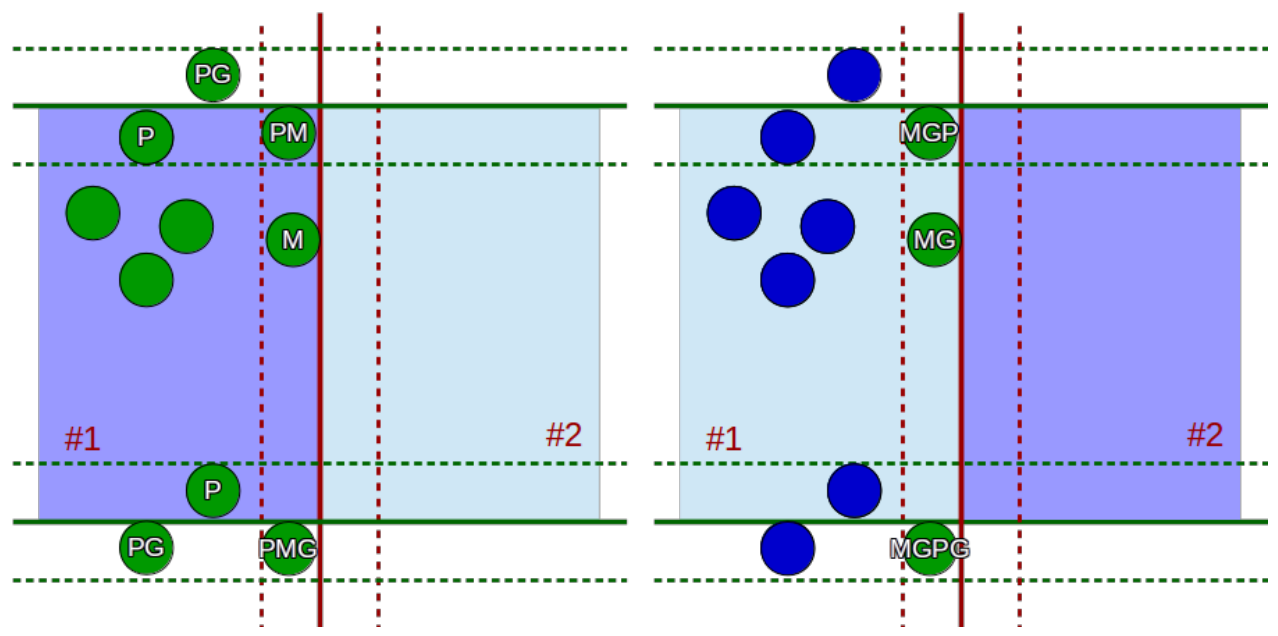

Figure C.6: Sketch of communication with periodic boundaries (green lines) and domain boundaries (red lines). Dashed lines indicate the communication zone with respect to the boundary. Particle types and explanations are listed in Tab. C.1.

It is possible that a particle seems to be an $R$-particle w.r.t. a certain periodic boundary, but a $P$-particle w.r.t. to another. To keep track how a particle is related to all sets of periodic boundaries, a periodic complexity is introduced, $\mathscr{C}_{p}$. This periodic complexity is not a scalar, in contrast to $\mathscr{C}$, but a vector with the size of the number of sets of periodic boundaries. This is because in the local communication a fixed domain shape is taken, while the number of periodic boundaries is never fixed. Each entry in $\mathscr{C}_{p}$ describes how the particle is related to this boundary, see Table C.2. A visual representation of the periodic complexity is given in Fig. C.7. Extra flexibility is introduced in this complexity to enable boundary specific interactions. In the specific case of a maser boundary, particles with value 3 are disabled from interacting with the maser boundary. The relation between $\mathscr{C}_{p}$ and the type of particle can be determined as follows. If all values of $\mathscr{C}_{p}$ are positive and at least one value equal to 1 , then the particle is a $P$-particle. If the particle contains any negative value then the particle is a $P G$-particle.

\begin{tabular}{|l|l|}
\hline $\mathscr{C}_{p_{i}}$ & Definition \\
\hline 2 & A particle that does not interact with the boundary. \\
1 & A real particle in the communication zone of the boundary. \\
-1 & A ghost particle in the communication zone of the boundary. \\
-2 & A ghost particle outside the communication zone of the boundary. \\
3 & Special case of a $P$-particle, requires a boundary specific action. \\
-3 & Special case of a $P G$-particle, requires a boundary specific action. \\
\hline
\end{tabular}

Table C.2: The definition of the periodic complexity values with respect to a given periodic boundary $i$. The value 3 and -3 are a special cases that enable boundary specific actions such as the Maser. 


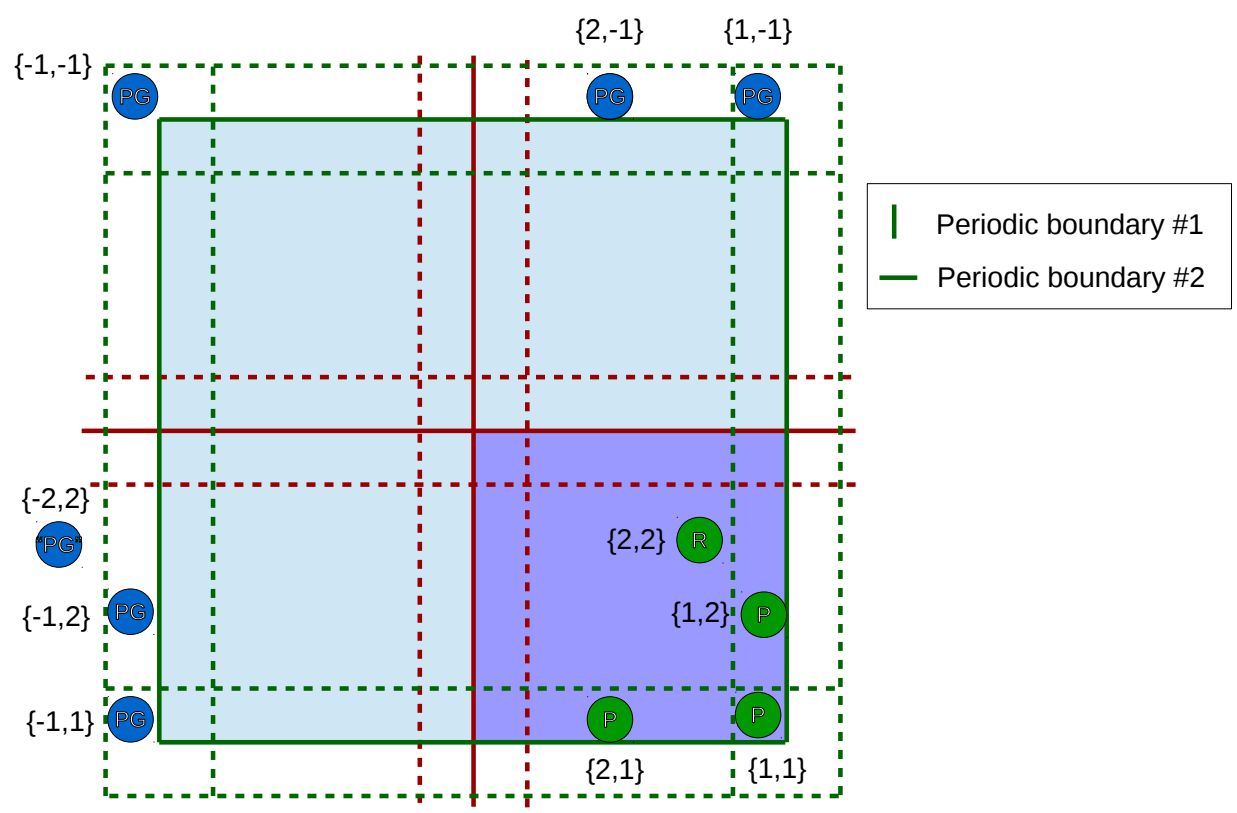

Figure C.7: Examples of the periodic complexity $\mathscr{C}_{p}$ for a situation with horizontal periodic boundaries and vertical periodic boundaries. Note that the ghost of the $R$-particle is flagged as " $P G$ "-particle, because it should not exist.

By introducing the periodic complexity, particles are aware of what type their type. However, the connection between $P G$-particles and $P$-particles still need to be made, especially because a $P$-particle can have ghosts in different domains as depicted in Fig. C.6. Therefore bookkeeping lists $\mathscr{P}$ and $\mathscr{H}$ are introduced. The list $\mathscr{P}$ keeps track of all $P$ particles located on the current domain $D_{i}$. If a $P$-particle has a ghost on domain $D_{j}$ it is listed in $\mathscr{P}_{i j}$. Similarly, if a $P G$-particle is located in domain $D_{i}$ and the original particle is located in $D_{j}$, it is listed in $\mathscr{H}_{i j}$. Operations on both $\mathscr{P}$ and $\mathscr{H}$ are designed such that

$$
\mathscr{P}_{i j}=\mathscr{H}_{j i}
$$

removing any need to perform a particle search. Note that particle with periodic complexity $\{1,1\}$ in Fig. C. 7 has three ghosts and is therefore listed three times in $\mathscr{P}$. Generation of ghost particles is relatively, given the periodic complexity of a $P$-particle. Obtaining all ghost particles is done by finding all permutations of values 1 in $\mathscr{C}_{p}$ to negative values -1 . As example see Fig. C. 7 where a $P$-particle with complexity $\{1,1\}$ has three ghosts, $\{-1,1\},\{1,-1\}$ and $\{-1,-1\}$.

One particular problem with introducing $P G$-particles is the shift in position. Consider a periodic boundary with the left boundary located at $x_{l}=0$ and the right boundary 
located at $x_{r}=100$. Now if a $P$-particle $p_{1}$ has a position of $x_{p}=1 \times 10^{-16}$ then the ghost position of this particle would be $x_{p g}=x_{r}+x_{p}$. Due to a finite numerical precision this would be rounded off to $x_{p g}=100$. Implying the ghost particle is a $P$-particle. It is therefore crucial for a stable algorithm that the position with respect to the periodic boundary is always computed with the original position of the $P$-particle. The periodic complexity of the $P G$-particles is therefore always updated using $\mathscr{C}_{p}$ of the $P$-particle and not based on the position of the ghost particles.

With the introduction of $M$-particles and $P$-particles, $P M$-particles are also introduced, particles that are both interacting with a periodic boundary and with a communication boundary, see Fig. C.6. This introduces a problem in which order the system is flagging particles and who is taking control of the particles. In this algorithm the local communication first performs a new particle check resulting in a list of $M-M G$ particles, both listed in $\mathscr{B}$ and $\mathscr{G}$ respectively. Next, the periodic communication yields $P$ - and $P G$-particles, listed in $\mathscr{P}$ and $\mathscr{H}$, respectively. Considering a $P M$-particle, the first step generates $P M$ - and $P M G$-particles listed in $\mathscr{B}$ and $\mathscr{G}$. The periodic step lists $P G M^{*}$ - and $P G M G^{*}$-particles in lists $\mathscr{P}$ and $\mathscr{H}$. Note that these particles are not listed in $\mathscr{B}$ and $\mathscr{G}$, although they are interacting with a communication boundary. Therefore, the periodic communication algorithm needs to deal with the local communication of these particles. Note that if the evaluation of particle type is done in the alternative order, $P^{*} M G$ - and $P G^{*} M G$-particles are introduced, i.e. periodic particles not listed in $\mathscr{P}$ and $\mathscr{G}$. These particles are considerably harder to update than $P G M *$-particles and $P G M G^{*}$-particles, due to shift in position.

This section has introduced a mesh-independent periodic communication structure that allows a wide variety of periodic boundaries. The structure of communication is very similar to the structure of the local communication. Ghost particles and communication zones, and bookkeeping lists $\mathscr{P}$ and $\mathscr{H}$ are introduced. A difference is the periodic complexity $\mathscr{C}_{p}$ which is vector instead of a scalar. Furthermore flexibility is build into this periodic complexity that allows for special actions which are boundary dependent. The communication of $P G M^{*}$ - and $P G M G^{*}$-particles is solely done by the periodic communication. Detailed algorithm of the implementation of this structure can be found in section C.2.4.

\section{C.2.3. INSERTION AND DELETION}

One of the most important operations in a DPM simulation is insertion and deletion of particles. There are many ways particles are added to the simulation: manually by the user at the start up of the simulation, insertion boundaries or the communication boundaries that add ghost particles. A clear distinction between the former two options and the latter option can be made. A ghost particle is not a unique particle, but a copy of a unique particle and more importantly, a ghost particle will always be added locally. When adding a unique particle, the location of this particle might be generated randomly and each core might generate a different set of random positions. To make sure only one particle is generated, the root generates a particle and sends the data of this particle to the other processors. Based on the location all processors evaluate if they have to add the particle. If they have to add the particle then they will add the particle, otherwise they will ignore the request. When a particle is added which increases the largest interaction 
radius the communication zones need to increase their interaction distance $I$, triggering an evaluation step that looks for new $M$ - and $P$-particles. Another important difference is that when adding a unique particle, it also generates a unique ID. This is not the case for a ghost particle.

Deleting particles generally is done with a deletion boundary. This boundary checks any particle, including ghost particles, if they need to be destroyed based on their position. The particles, however, will not be destroyed directly, in case they are close to a communication boundary. These particles first need to be flushed from the communication lists to avoid memory problems. After flushing them from the bookkeeping lists the particles are destroyed.

\section{C.2.4. Parallel algorithm implementation}

This section focuses on the the details of the implementation in MercuryDPM using the structure and definitions discussed in section C.2.1 and section C.2.2.

The communication procedure takes place after the positions and velocity of real particles have been updated and it consists of a seven-step process, see Alg. 1 . The first step is to update the positions and velocities of all $M G$-particles. The second step is to evaluate the particle type of all $M$ - and $M G$-particles and take the proper actions. The third and fourth step updates the $P G$-particles and the the particle type of all $P G$-and $P$-particles, respectively. Sometimes it is necessary to delete particles, however deletion can not be done straight away. Some ghost particles are both located in the data structure of the periodic boundaries and the data structure of the local communications. Instead, particles that have to be removed are flagged for removal and deletion is postponed until all particles are updated and, if necessary, removed from the bookkeeping lists. Deleting these particles is straightforward so no details are given. The last two steps introduce new $M$ - and $M G$-particles and new $P$ - and $P G$-particles. At the end of Alg. 1 all particles now have neighbours which are up to date such that the contact interactions can be computed in Eq. (C.3). The algorithms for updating particles and finding new particles will be discussed in detail in order.

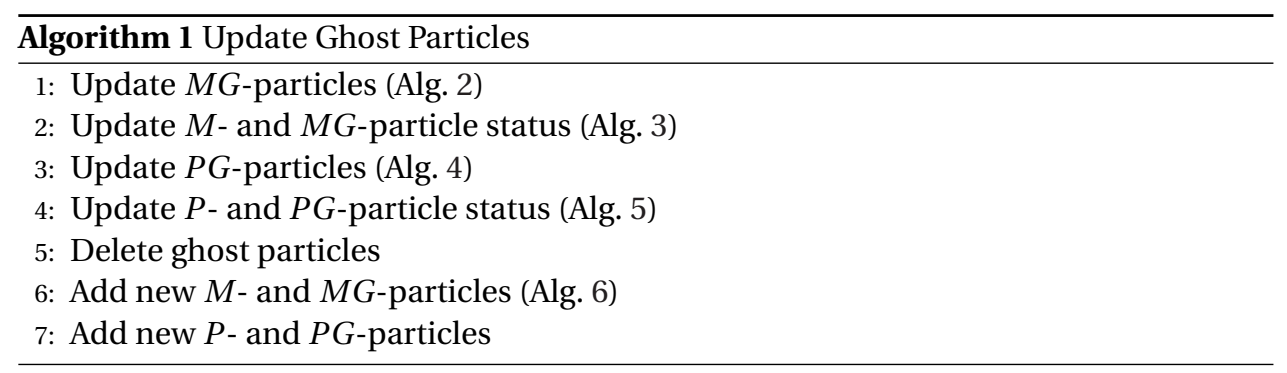

\section{UPDATE $M G$-PARTICLES}

The procedure for updating the position and velocities of $M G$-particles follows Alg. 2 . Consider domain $D_{i}$ and the set of neighbouring domains $D_{n}\left(D_{i}\right)$. For each neighbour domain $D_{j} \in D_{n}\left(D_{i}\right)$ a data structure data_ij is generated that contains $\boldsymbol{v}^{n+\frac{1}{2}}$ and $\boldsymbol{x}^{n+1}$ of all $M$-particles in $\mathscr{B}_{i j}$. After the data has been collected a communication step is 
required. Communication using the MPI protocol requires a sending and a receiving action. The standard send and receive requests are called "blocking" communications, because all other communications are blocked until that specific send and receive request has been resolved. This is highly inconvenient when multiple domains have to talk to many other domains and hence a different type of communication is used, asynchronous communication. In this type of communication a send or receive request is made, and it will be pending until a resolve request is made. When all send and receive requests of $d a t a \_i j$ are made, a synchronise step is called where these pending requests are resolved simultaneously. After the synchronisation step the receiving data can then be used to update the corresponding $M G$-particles in $\mathscr{G}_{i j}$. Note that because of Eq. (C.8), no particle search has to be performed.

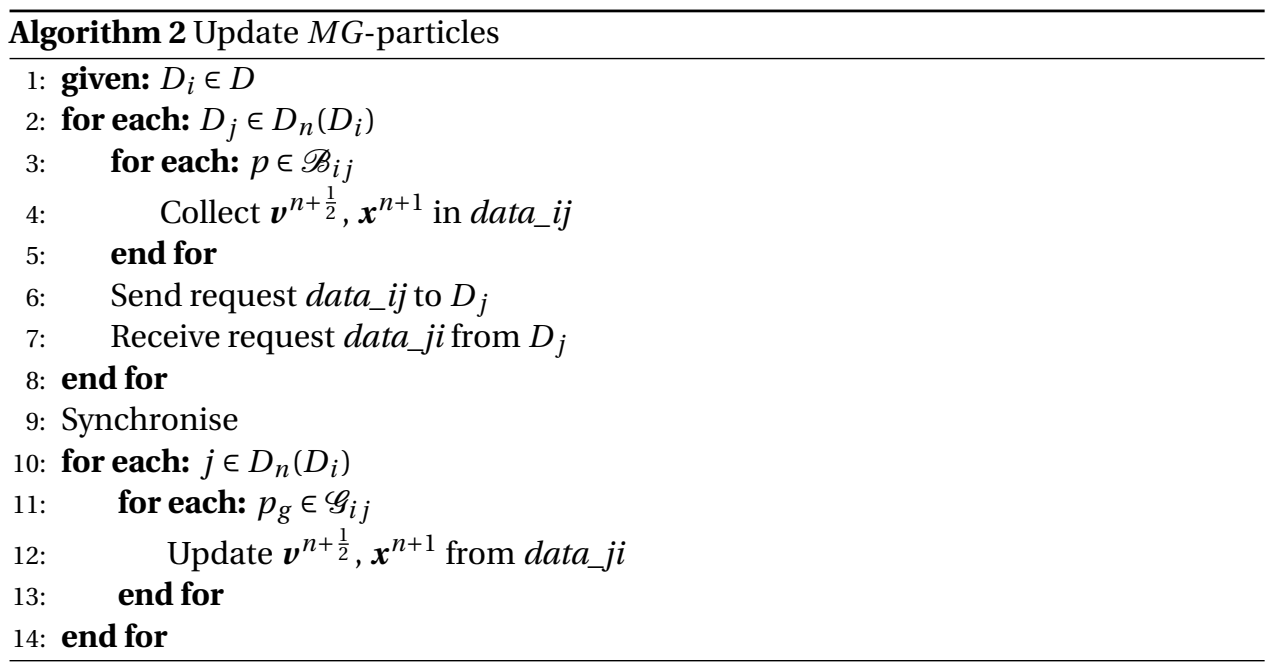

\section{UPDATE $M$ - AND $M G$-PARTICLE STATUS}

After the $M G$-particles have received their new position and velocities, the status of all $M$ - and $M G$-particles require a re-evaluation. Each domain checks the particles in the lists $\mathscr{G}$ and $\mathscr{B}$ for any type changes. The algorithm that updates the lists is given in Alg. 3 . First the list of $M$-particles is considered, $\mathscr{B}$. There are two options that can occur. Firstly the particle might have moved out of the domain, if that is the case the particle needs to be deleted. Secondly the complexity of the particle might have changed. This could be because the particle moved away from the boundary, or because the particle moved into a new boundary. This particle is then flagged as $R$-particle and removed from the list. If it is still in the communication zone it will be reintroduced when finding new $M$-particles (see Alg. 1). The boundary list of $M G$-particles, $\mathscr{G}$ is updated in a slightly different manner compared to $\mathscr{B}$. First a check is done to see if the ghost particle still belongs to the neighbour domain $D_{j}$. If that is not the case then either the ghost particle moved to $D_{i}$, or it moved away from $D_{j}$ to another domain. In the first case the $M G$-particle becomes an $R$-particle and in the latter case the $M G$-particle needs to be removed. Finally, if the complexity of the corresponding real particle on domain $D_{j}$ changes, the real particle 
will be removed from the communication lists and reintroduced in a later step. Therefore, the ghost also has to be removed from $\mathscr{G}$.

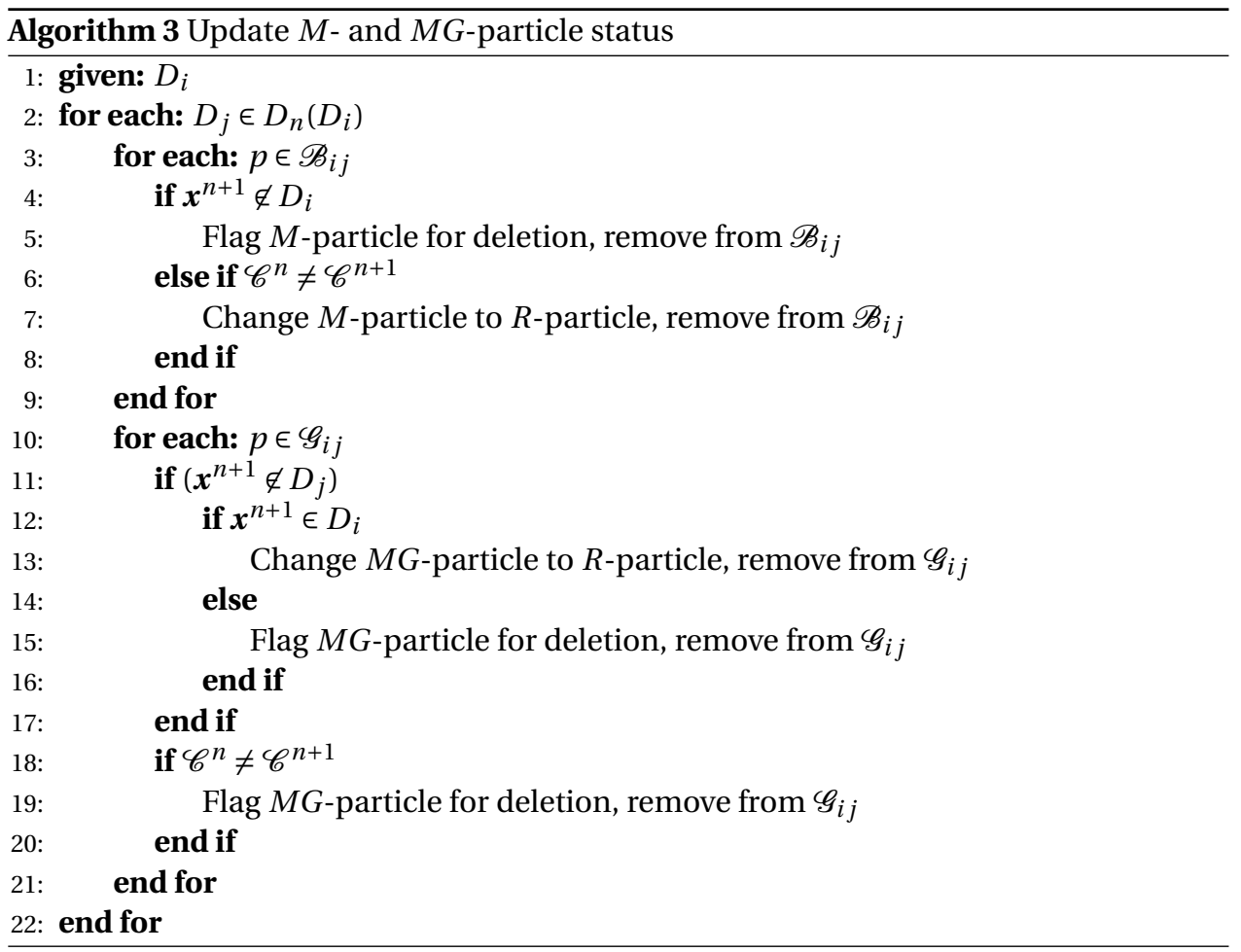

\section{UPDATE $P G$-PARTICLES}

Updating the $P G$-particles is similar to updating $M G$-particles with a few exceptions. The algorithm is given in Alg. 4 gives an abstract of the implementation. First the positional data is collected from all $P$-particles. However, the $P$-particles on a given domain which have a $P G$-particle on the same domain can skip this step. No communication is required in this case as the domain can already access the information. After all data has been collected and all send and receive requests have been queued, the communication is synchronised and the actual $P G$-particle update can take place. The $P G$-particles with a corresponding $P$-particle on the same domain can access $\boldsymbol{v}^{n+\frac{1}{2}}$ and $\boldsymbol{x}^{n+1}$ of the periodic particle listed in $\mathscr{P}_{i j}$. Based on the periodic complexity of the $P G$-particle, the velocity and position of the original particle can be shifted to the correct position and velocity of the ghost, $\boldsymbol{v}_{g}^{n+\frac{1}{2}}, \boldsymbol{x}_{g}^{n+1}$. After shifting these values to the velocity and position of the ghost the $P G$-particle can receive its update. Similar update is required for all other ghost particles, with the exception that the data is not directly from $\mathscr{P}_{i j}$, but from the received data $d a t a \_i j$. 


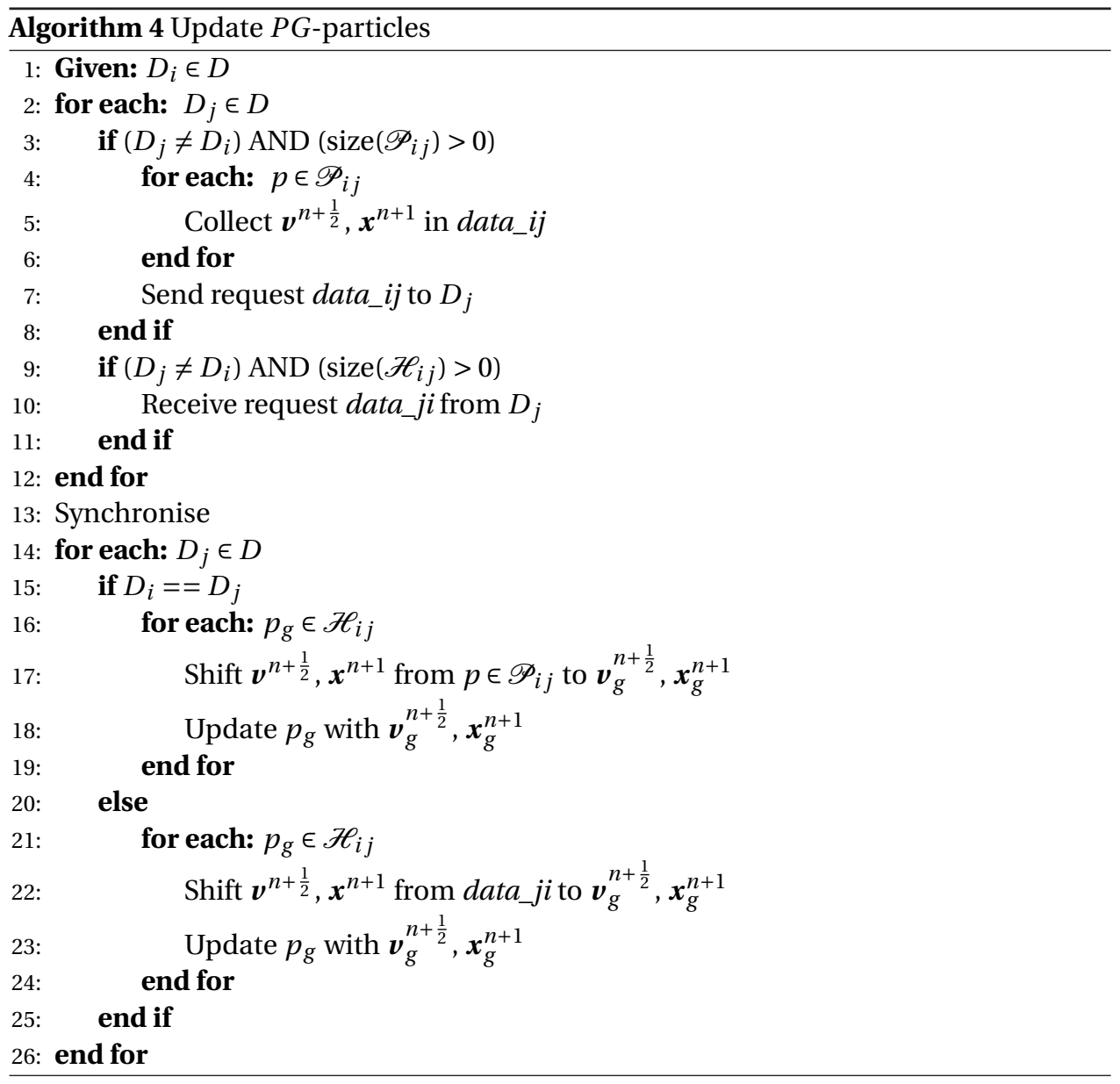




\section{UPDATE $P$ - AND $P G$-PARTICLE STATUS}

Updating the type of particles is shown in Alg. 5. The periodic complexity of all real particles listed in $\mathscr{P}$ is computed based on the position. Next, the periodic complexity of all real $P$-particles is evaluated. If a change is detected w.r.t the previous time step then either the particle remains real, or becomes a ghost. In the former case the particle is flagged as $R$-particle and a boundary specific action can be performed, required i.e. in the maser boundary. If the particle becomes a ghost it will be flagged for deletion. At the same time the $P$-particle could be an $M$-particle. If the particle switches from $P M$-particle to $P M G$-particle then Alg. 2 will flag the particle for deletion and hence this particle has to be removed from $\mathscr{P}$. Alternatively, the $P$-particle could be an $M G$-particle that has moved out of the communication zone of the domain. Then Alg. 3 will flag the particle to be deleted and therefore this particle will also be removed from $\mathscr{P}$.

Ghost particles compute $\mathscr{C}_{p_{g}}^{n+1}$ based on the current periodic complexity of the corresponding real particle and the periodic complexity of both ghost and real particle of the previous time step,

$$
\mathscr{C}_{p_{g}, i}^{n+1}=\left|\mathscr{C}_{p, i}^{n+1}\right| \operatorname{sign}\left(\mathscr{C}_{p, i}^{n} \mathscr{C}_{p, i}^{n+1} \mathscr{C}_{p_{g}, i}^{n+1}\right)
$$

Here $\left|\mathscr{C}_{p}^{n+1}\right|$ gives the magnitude of the new value, see Tab. C.2. If the real particle crossed a periodic boundary it switches sign, captured by the term $\mathscr{C}_{p, i}^{n} \mathscr{C}_{p, i}^{n+1}$. Multiplying this by the sign of the current ghost particle gives the new periodic complexity of the ghost. If the ghost particle has become real, there are two options. Either the particle is an $M G^{*}$-particle, in that case the particle will be flagged for deletion as this is not actually a real particle, otherwise it truly is a real particle and it will be flagged as $R$-particle and removed from $\mathscr{H}_{i j}$. The last update step consists of updating the $P G M^{*}$-and $P G M G^{*}$ particles. If their status change they will be flagged for deletion.

\section{FIND NEW $M$ - AND $M G$-PARTICLES}

As the status of all particles are now updated, it is time to introduce new $M$-particles and their corresponding $M G$-particles that either have entered the communication zone, or have been removed in the status update. For that reason two new lists are introduced, $\mathscr{S}_{i j}$ and $\mathscr{I}_{i j}$. The first list contains newly found particles and the second structure contains interactions between the newly found particles and their neighbour particles. The full algorithm is shown in Alg. 6. Particles that have been flagged as $R$-particles but have a position within the communication zone of the boundary are considered to be new particles. Newly found interactions are interactions in which either $p_{1}$ or $p_{2}$ is in $\mathscr{S}_{i j}$ and the other particle is in either $\mathscr{S}_{i j}, \mathscr{B}_{i j}$ or $\mathscr{G}_{i j}$. The next step is to add the corresponding ghost particles. First the receiving domains need to know how many particles and interactions they are going to receive, which is the start point for sending the actual particle and interaction data. After the synchronise step the strictly necessary particle and interaction data are copied to Sdata and Idata, respectively. After all data is gathered into a data that MPI can communicate, the send and receive requests are made and resolved at the synchronisation step. The ghost particles can now be created together with their interactions. Note that the interactions only contain the ID's of the interaction particle. It is a-priori not clear where the second particle is located in memory and therefore each 


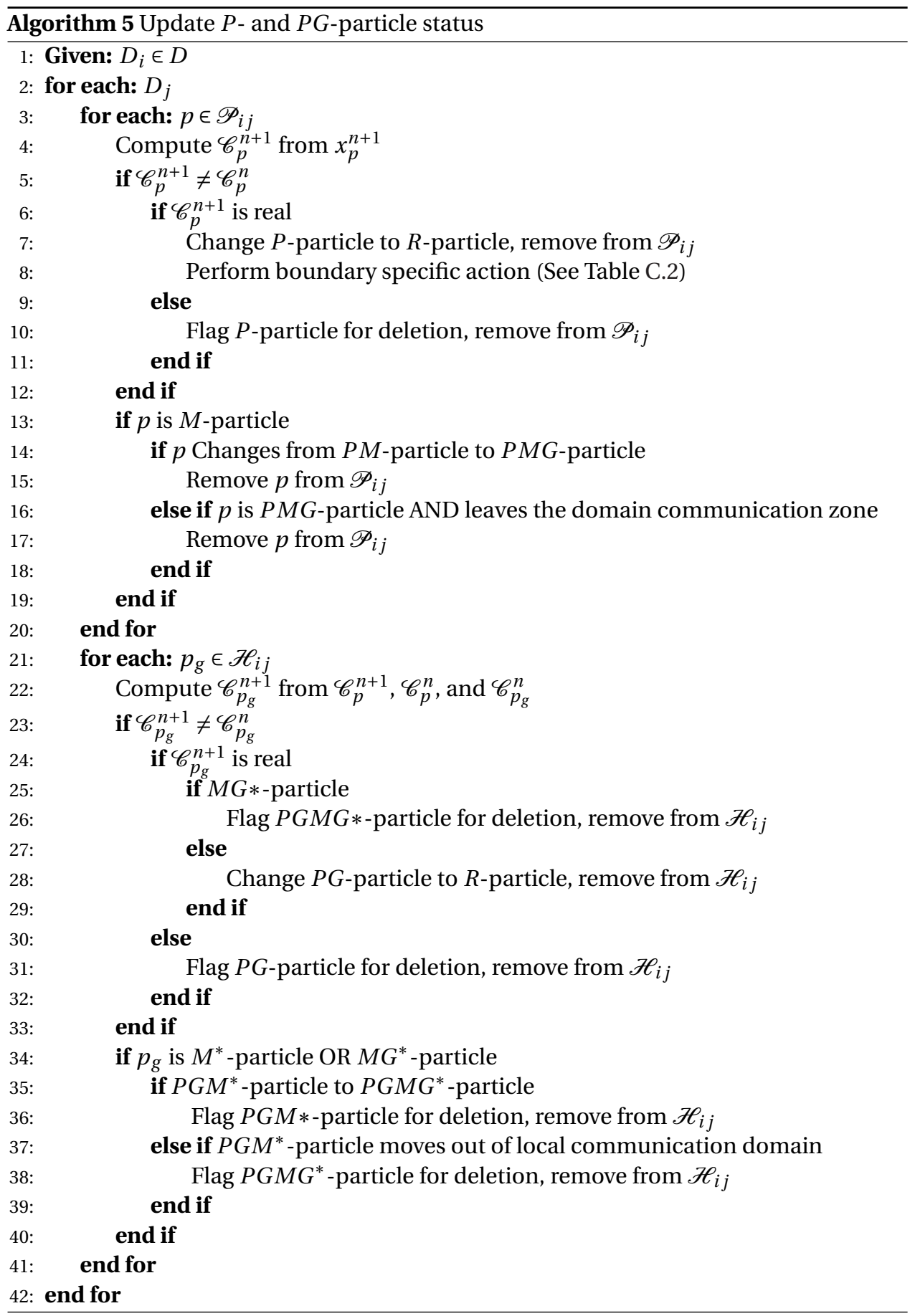


interaction requires a particle search. This search is based on the hierarchical hGrid [6] similar to finding contacts in the general simulation.

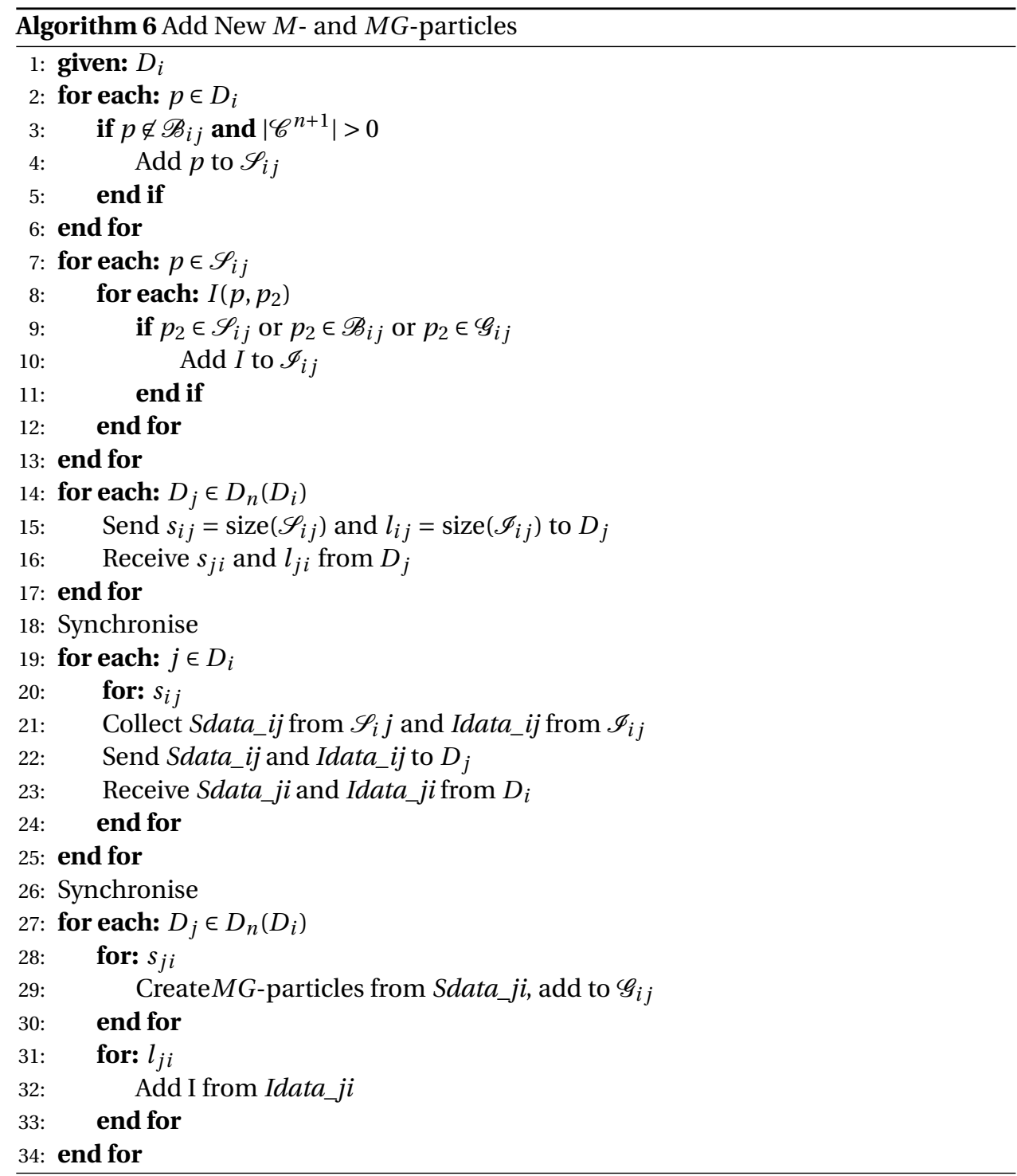

Find NEW $P$ - AND $P G$-PARTICLES

Finding new $P$ - and $P G$-particles is done in a similar manner as Alg. 6 with a few notable implementation differences. When a $P$-particle is found on $D_{i}$, all possible $P G$-particles positions are generated. Based on these ghost positions, the target domain $D_{j}$ is computed which is a-priori not known. It is possible that the target domain is the same as $D_{i}$ which would not require a communication step, instead ghosts can be directly cre- 
ated. Before communicating the newly found $P$-particles, all domains need to know how many particles which domain is receiving and sending, this global communication step is significantly different from the local communication in Alg. 6, where the domains only communicate with neighbour domains.

\section{C.3. Results}

Estimation of the algorithm's performance is done by investigating the weak scalability. Weak scaling is determined by increasing the overall problem size, while keeping the problem per core constant. A rotating drum, see Fig C.1, is therefore an ideal setup as it is trivial to extend the simulation in axial direction.

Simulations are performed with a bidisperse mixture with a fractional polydispersity of $5 \%$. The ratio between the mean particle diameters of the large and small particles is $d_{l} / d_{s}=1.5$. The scaling of the contact detection algorithm is influenced by polydispersity [6]. Therefore, single core simulations are performed to estimate the scaling law between simulation time $T_{s}$ and number of particles $N_{p}$. The results are shown in Fig. C.8(a), yielding a power law of

$$
T_{s}^{S}\left(N_{p}\right)=a N_{p}^{b}
$$

where subscript $S$ implies the simulation time on a single core. The coefficients in the power law are $a=1.0818$ and $b=1.17$.

The efficiency of the parallel code is defined as the ratio between the estimated simulation time on one core, compared to the cumulative simulation time of the parallel simulation,

$$
E=T_{s}^{S} /\left(T_{s} N_{c}\right)
$$

where $N_{c}$ is the number of cores used. The efficiency of simulations on a single node with 36 processors (up to 72 when hyperthreading is used) is shown in Fig. C.8(b). As the number of cores increases, the efficiency reduces. An apparent linear trend can be observed up to roughly $E \approx 0.6$. When enabling hyperthreading, the efficiency even lowers to $E \approx 0.4$. However, it does seem to be independent of the number of cores in this regime. In Fig. C.8(c) the efficiency is shown when simulating on multiple nodes without hyperthreading, where different colours indicate a different number of cores per node. As expected there is an initial linear downwards trend up till roughly 60 cores. For higher number of cores the performance seems to stabalise around an efficiency of $E \approx 0.6$. A constant efficiency implies the algorithm scales similar to a single core simulation (with a prefactor of $E$ ).

A possible explanation for the observed decrease in efficiency could be due to inefficient load balancing. The particles in the rotating drum can move freely between cores. Specifically, in long rotating drums particles move away from the centre towards the wall of the simulation, increasing the computational cost on these cores [24].

\section{C.4. Conclusion AND Discussion}

A parallel algorithm has been developed and implemented in the open-source software MercuryDPM. A user specified 3D Cartesian mesh decomposes a DPM simulation into 

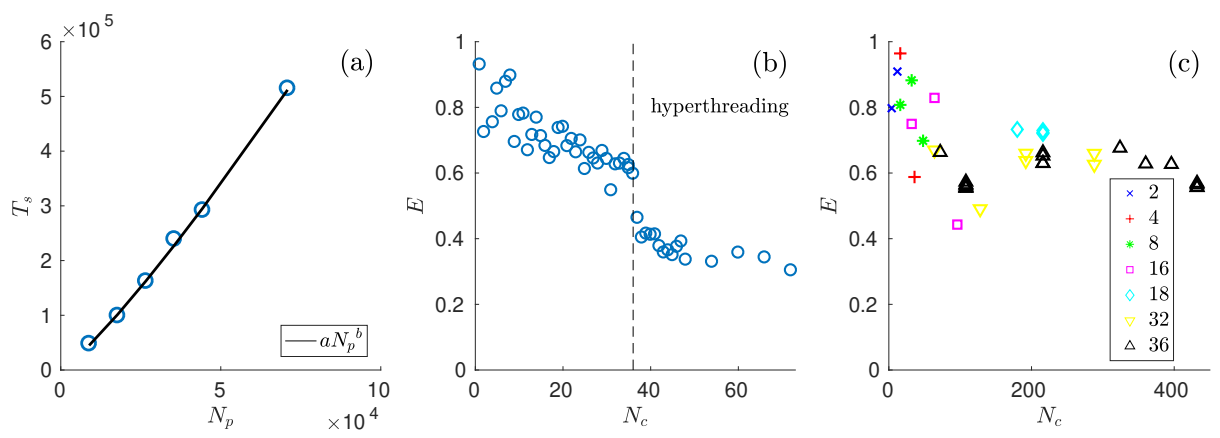

(c)

Figure C.8: (a) Single core simulations of different drum lengths, parameters of scaling fit are $a=1.0818$ and $b=1.17$ (b) Efficiency of the parallel algorithm on a single node with a maximum of 36 physical cores and 72 cores including hyperthreading. (c) Efficiency of the parallel algorithm on more than 1 node without hyperthreading. Different symbols indicate the number of cores per node.

equally sized domains. The local communication between neighbouring boundaries is done by employing ghost particles, which contain up-to-date information of real particles on neighbouring domains. Ghost particles are also used for specialised boundaries such as periodic and maser boundaries. However, these boundaries perform global communication, as the target domain in these cases is not know a-priori. Both the local and global communication algorithms are discussed in great detail, such that this work can be used as manual for future developers of MercuryDPM.

The weak scaling of the algorithm is investigated to estimate its performance. This scaling shows an initial decrease in performance of $40 \%$ up to 60 cores, while simulations with more cores show no further decrease. Therefore, the algorithm performs very efficient for large simulations. The decrease in efficiency could be caused by inefficient load balancing which could be tested by placing walls inside the drum at each core interface. The walls prevent particles from traveling to other domains, but keep communication between domains. It is recommended to investigate the strong scaling of the algorithm such that the optimal ratio between domain volume and communication area can be determined. Furthermore, the performance of periodic and maser boundaries has yet to be determined.

The current implementation works well for evenly distributed particle systems. However, for different systems load balancing between cores could become an issue. To optimise the algorithm for all systems, automatic load balance is mandatory. One possibility to enhance load balancing is by implementing an adaptive mesh of different sized domains. Another option is to combine the current MPI implementation with a local openMP implementation [18]. The implementation uses a fixed interaction distance at domain boundaries for local communication. This interaction distance is defined as twice the largest particle in the system. The algorithm could be improved by making this distance depend on the hierarchical contact detection grid.

The data generated by massively parallelised systems could become a burden to analyse. As a recommendation, the output of MercuryDPM in these cases should switch from discrete particle data to coarse grained continuum fields, for details see Ref. [25]. 
As an additional option, user specified regions of interest should still be able to output discrete data.

\section{REFERENCES}

[1] O. I. Imole, D. Krijgsman, T. Weinhart, V. Magnanimo, B. E. C. Montes, M. Ramaioli, and S. Luding, Experiments and discrete element simulation of the dosing of cohesive powders in a simplified geometry, Powder Technology 287, 108 (2016).

[2] P. Jop, Y. Forterre, and O. Pouliquen, A constitutive law for dense granular flows, Nature 441, 727 (2006).

[3] G. Ning and Z. Jidong, A coupled FEM/DEM approach for hierarchical multiscale modelling of granular media, International Journal for Numerical Methods in Engineering 99, 789 (2014).

[4] T. Weinhart, D. R. Tunuguntla, M. P. van Schrojenstein Lantman, A. J. van der Horn, I. F. C. Denissen, C. R. Windows-Yule, A. C. de Jong, and A. R. Thornton, Mercurydpm: A fast and flexible particle solver part A: Technical advances, (Springer Singapore, Singapore, 2017) pp. 1353-1360.

[5] P. A. Cundall and O. D. L. Strack, A discrete numerical model for granular assemblies, Géotechnique 29, 47 (1979).

[6] V. Ogarko and S. Luding, A fast multilevel algorithm for contact detection of arbitrarily polydisperse objects, Computer Physics Communications 183, 931 (2012).

[7] D. Krijgsman, V. Ogarko, and S. Luding, Optimal parameters for a hierarchical grid data structure for contact detection in arbitrarily polydisperse particle systems, Computational Particle Mechanics 1, 357 (2014).

[8] I. F. Denissen, T. Weinhart, A. Te Voortwis, S. Luding, J. Gray, and A. Thornton, Bulbous head formation in bidisperse shallow granular flow over an inclined plane, Journal of Fluid Mechanics 866, 263 (2019).

[9] S. Roy, S. Luding, and T. Weinhart, A general(ized) local rheology for wet granular materials, New Journal of Physics 19, 043014 (2017).

[10] V. Smilauer, E. Catalano, B. Chareyre, S. Dorofeenko, J. Duriez, N. Dyck, J. Elias, B. Er, A. Eulitz, A. Gladky, and et al., Yade Documentation 2nd ed (Zenodo, 2015).

[11] EDEM Simulator, Edem simulator, https://www.edemsimulation.com, accessed: 2018-01-02.

[12] 3DEC, Distinct-Element Modeling of Joined and Blocky Material in 3D, 3dec.. https : //www . itascacg. com, accessed: 2018-01-02.

[13] Newton, Newton advanced conveyor technologies, http://www.demsoftware. net/, accessed: 2018-01-02. 
[14] Nvidia, Nvidia Tesla V100 datasheet, https://www.nvidia.com/en-us/ data-center/tesla-v100/, accessed: 2018-01-17.

[15] L. Clarke, I. Glendinning, and R. Hempel, The mpi message passing interface standard, in Programming Environments for Massively Parallel Distributed Systems, edited by K. M. Decker and R. M. Rehmann (Birkhäuser Basel, Basel, 1994) pp. 213218.

[16] E. Gabriel, G. E. Fagg, G. Bosilca, T. Angskun, J. J. Dongarra, J. M. Squyres, V. Sahay, P. Kambadur, B. Barrett, A. Lumsdaine, R. H. Castain, D. J. Daniel, R. L. Graham, and T. S. Woodall, Open mpi: Goals, concept, and design of a next generation mpi implementation, in Recent Advances in Parallel Virtual Machine and Message Passing Interface, edited by D. Kranzlmüller, P. Kacsuk, and J. Dongarra (Springer Berlin Heidelberg, Berlin, Heidelberg, 2004) pp. 97-104.

[17] R. Thakur, R. Rabenseifner, and W. Gropp, Optimization of collective communication operations in mpich, The International Journal of High Performance Computing Applications 19, 49 (2005).

[18] R. Berger, C. Kloss, A. Kohlmeyer, and S. Pirker, Hybrid parallelization of the liggghts open-source dem code, Powder Technology 278, 234 (2015).

[19] W. C. Swope, H. C. Andersen, P. H. Berens, and K. R. Wilson, A computer simulation method for the calculation of equilibrium constants for the formation of physical clusters of molecules: Application to small water clusters, The Journal of Chemical Physics 76, 637 (1982).

[20] S. Luding, Introduction to discrete element methods, European Journal of Environmental and Civil Engineering 12, 785 (2008).

[21] A. D. Renzo and F. P. D. Maio, Comparison of contact-force models for the simulation of collisions in dem-based granular flow codes, Chemical Engineering Science 59, 525 (2004).

[22] R. Courant, K. Friedrichs, and H. Lewy, Über die partiellen differenzengleichungen der mathematischen physik, Mathematische Annalen 100, 32 (1928).

[23] A. Podlozhnyuk, S. Pirker, and C. Kloss, Efficient implementation of superquadric particles in discrete element method within an open-source framework, Computational Particle Mechanics 4, 101 (2017).

[24] M. Arntz, W. K. den Otter, H. Beeftink, R. Boom, and W. J. Briels, The influence of end walls on the segregation pattern in a horizontal rotating drum, Granular matter 15, 25 (2013).

[25] T. Weinhart, A. R. Thornton, S. Luding, and O. Bokhove, From discrete particles to continuum fields near a boundary, Granular Matter 14, 289 (2012). 



\section{ACKNOWLEDGEMENTS}

This thesis would not have been possible without the help and support of other people. Firstly, I would like to thank my promotor Anthony Thornton and co-promotor Stefan Luding. Anthony, you gave me a lot of freedom to pursue my own research interests within the project, but you also kept me in line such that I actually finished a few of them. Furthermore, I would like to thank you for giving me the opportunity to get familiar with software development, resulting in a parallelised MercuryDPM. I have enjoyed the many conference we have visited together. Stefan, thank you for the interesting and deep scientific discussions we've had. You have often steered me in the right direction, helped putting my thoughts clearer on paper and prevented me from reïnventing the wheel.

This thesis builds further on the work of previous researchers which I would like to thank. Kasper, je hebt me geïntroduceerd in het onderzoeksonderwerp van dit proefschrift. Ik vind dat we een goed onderzoeksteam zijn en wil je graag bedanken voor de prettige samenwerking. Thomas, a huge part of the data analysis in this thesis would not have been possible without the new and incredibly fast MercuryCG tool you have developed and implemented. François, I am grateful for the discussions we had on single particle segregation and that you have shared your simulation results so freely. I would like to thank Andrew Hazel for the great support with the oomph-lib software. Sudeshna, thank you for the nice collaboration on the rheology project.

I would like to thank all the colleagues I have worked with over the years. In particular I would like to thank Irana. Irana, we zijn tegelijkertijd begonnen en hebben altijd hetzelfde kantoor gedeeld. Het was niet alleen nuttig om de kennis tussen een werktuigbouwer en wiskundige uit te delen, ik vond het vooral heel gezellig en wens je veel succes toe met de afronding van jou promotie. Sylvia, ik kon altijd langskomen voor een gezellig praatje in goede tijden en mijn frustraties kwijt in slechte tijden. Bedankt voor alle hulp en vrolijkheid. Wouter, bedankt voor alle technische hulp gedurende de promotie en het helpen met het draaien en analyseren van de parallele simulaties. Bert, ik weet niet of ik mijn promotie had afgemaakt zonder de motivatie/inspiratie pot die je altijd gevuld hield. Yousef and Hongyang, It was really enjoyable to share the office with you.

Het volhouden van een promotie is niet mogelijk zonder afleiding. Ik zou graag iedereen van Messed Up willen bedanken voor de altijd gezellige activiteiten en trainingen. Mijn excuses aan de goalies voor alle frustratie die ik tijdens de training eruit sloeg. Ik wil Heren 1 bedanken voor alle leuke wedstrijden (en potjes taipan). Ik wil Age, Ties, Kyrian, Pieter, Anouk en Axel bedanken voor alle spelletjes, series en bier.

Mama en papa, jullie hebben altijd achter mij gestaan en zonder jullie was ik nooit zo ver gekomen. Evelien, jij bent altijd een stap verder in het leven. Bedankt voor het geven van een goed voorbeeld en alle bijbehoorde adviezen. Irene en Hugo, jullie zijn beiden ook bezig met een promotie en ik wens jullie veel succes met de afronding, ik heb er alle vertrouwen in. Bedankt, lieve Annet. 



\section{Curriculum Vitae}

\section{Marnix Pieter van Schrojenstein Lantman}

18-07-1988 Born in Velsen, the Netherlands.

\section{EDUCATION}

2000-2007 Atheneum,

De Nieuwe Veste, the Netherlands

2007-2011 B.Sc. in Mechanical Engineering

University of Twente, the Netherlands

2011-2014 M.Sc. in Mechanical Engineering,

Universiteit Twente, the Netherlands

Thesis: High Order Compact Difference Multigrid Solver for Elliptic Problems.

Supervisor: $\quad$ Prof.dr.ir. C. H. Venner

2014-2019 PhD candidate Multi Scale Mechanics

University of Twente, the Netherlands

Thesis:

A Study on Fundamental Segregation Mechanisms in Dense Granular Flows

Promotor: $\quad$ Prof.Dr. A. Thornton

Co-Promotor: $\quad$ Prof.dr.rer.-nat. S. Luding 


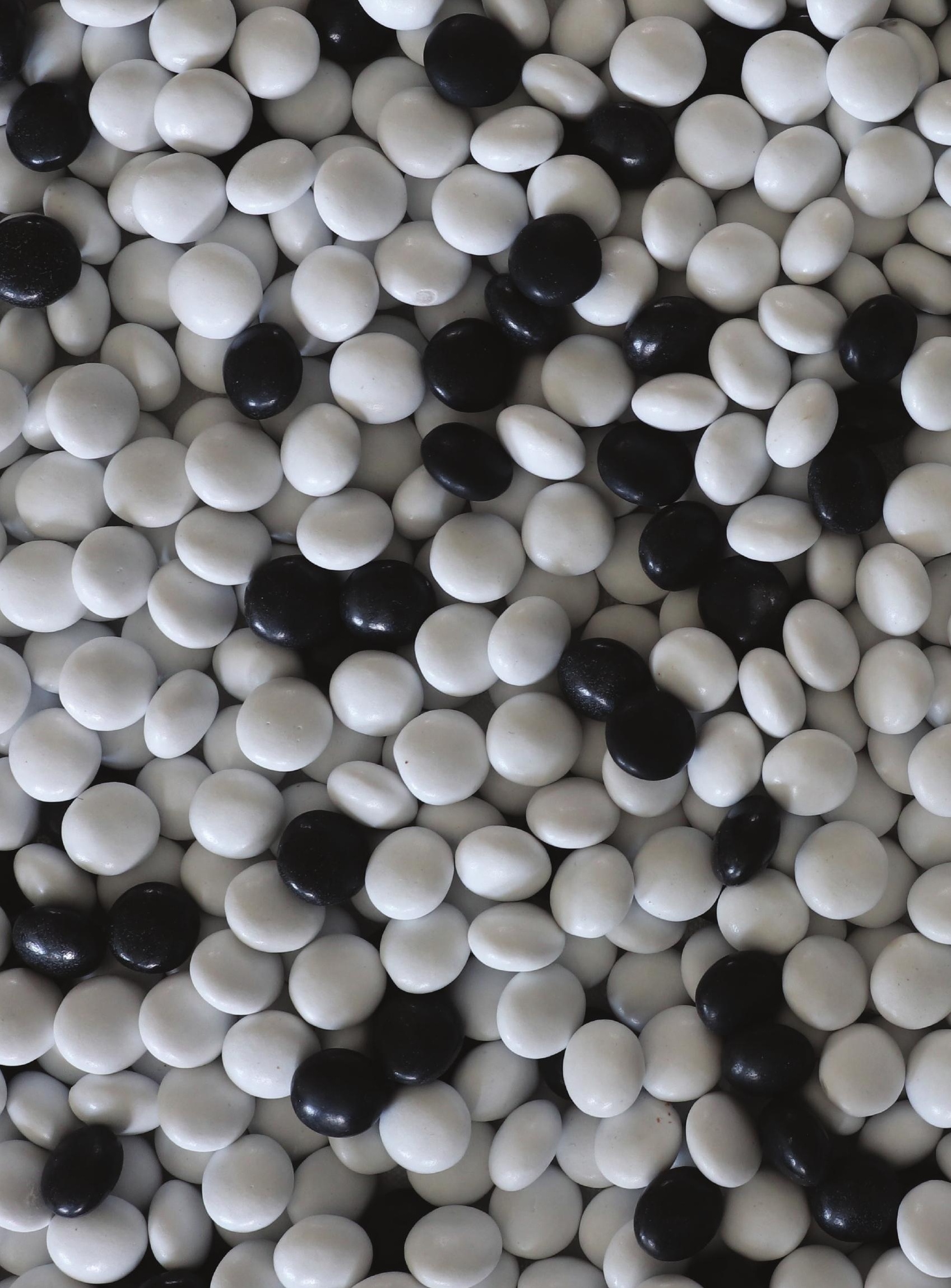

